\title{
The Development of a Low-Cost Microfluidic Magnetic Separation System
}

\author{
by
}

Geoffrey Smith

\begin{abstract}
A thesis submitted to Victoria University of Wellington in fulfilment of the requirements for the degree of Master of Science in Physics
\end{abstract}

Victoria University of Wellington 2009 


\begin{abstract}
Microfluidic systems show excellent promise for analytical applications, due to their ability to rapidly process minute sample quantities with high sensitivity. At the same time, functionalised superparamagnetic magnetic microbeads and nanoparticles have emerged as useful substrates for biomedical applications such as bioassays, fuelling research into tools for the manipulation of magnetic particles in microfluidic channels. This thesis describes the design, fabrication and evaluation of microfluidic systems for the separation of magnetic microbeads and nanoparticles. Microfluidic devices were produced in polydimethylsiloxane using a low-cost rapid prototyping process. Channels $300 \mu \mathrm{m}$ or greater in width were accurately reproduced using this method. Laminar flow was observed in the channels of these devices, allowing two-phase flow to be used for separation purposes. Magnetic field gradients of $25-500 \mathrm{~T} / \mathrm{m}$ were generated in the microchannels using either permanent magnets or soft magnetic materials. The performance of a permanent magnet-based separation system was evaluated, and it was found that the system could extract magnetic microbeads with an efficiency of up to $75 \%$. A limited ability to separate magnetic microbeads on the basis of magnetic moment and/or particle size was also demonstrated.
\end{abstract}




\section{Acknowledgements}

I would like to thank my supervisors, Dr. Gideon Gouws and Dr. Richard Tilley. Their guidance and continued enthusiasm have been invaluable in both the experimental and writing stages of this project.

Thanks to Billy Chooi for his earlier work, which provided an excellent foundation for the microfabrication component of this thesis. I would also like to thank him for the time spent passing on the skills he had obtained over the course of his project. I need to thank Soshan Cheong and Peter Ferguson for patiently teaching me the techniques used for nanoparticle synthesis and functionalisation, and for accommodating a physicist in their lab.

Further thanks go to David Flynn and Prof. Pablo Etchegoin for assistance with optical microscopy. Dr. Grant Williams and Nick Strickland provided access and supervision for VSM and SQUID magnetic measurements.

I would like to thank Nick Monahan and Kate McGrath for the use of their syringe pumps, and for useful discussion and advice. I am also grateful to Mark Hunter, Johnny McClymont and Chris Bumby for providing equipment used in microfabrication. A number of items of equipment were manufactured by Alan Rennie and Manu PouajenBlakiston, and I am grateful for their efforts and expertise.

I must also thank the SCPS for providing an excellent working and social environment, and for the myriad favours which could not be listed here. Finally, I wish to thank my friends and family for their enduring support and encouragement, and for providing an excuse to put the work aside every now and then.

This project was funded by a Victoria Master's (by thesis) Scholarship. Additional funding was provided from the Foundation for Research Science and Technology, through the New Economy Research Fund grant 'Magnetic Nanoparticles'. 
2.1.1 Diamagnetism and paramagnetism

2.1.2 Ferromagnetism and magnetic domains

2.1.3 Magnetic anisotropy

2.1.4 Monodomain and superparamagnetic materials

2.2 Magnetic nanoparticles and microbeads

2.2.1 Synthesis of magnetic nanoparticles

2.2.2 Synthesis of magnetic microbeads

2.2.3 Applications of magnetic nanoparticles and microbeads

\subsection{Microfluidics}

2.3.1 Lab-on-a-chip systems

2.3.2 Laminar flow

2.3.3 Diffusion

2.3.4 Device fabrication

2.3.5 Flow control

2.4 Forces on a magnetic particle

2.4.1 Gravity

2.4.2 Fluid drag

2.4.3 Magnetic force

2.4.4 Brownian motion

2.4.5 Particle agglomeration

2.4.6 Particle velocity 
3.1.3 Replica moulding in PDMS 63

3.1.4 Fluid connections 66

$\begin{array}{ll}\text { 3.1.5 Device sealing } & 68\end{array}$

3.1.6 Magnetic field gradient application $\quad 71$

$\begin{array}{ll}3.2 \text { Synthesis of magnetic nanoparticles } & 79\end{array}$

3.2.1 Thermal decomposition $\quad 79$

3.2.2 Manual purification $\quad 82$

3.2.3 Recapping of particles for use in aqueous solvents $\quad 84$

$\begin{array}{lr}3.3 \text { Flow control } & 85\end{array}$

3.3.1 Experimental setup $\quad 85$

$\begin{array}{ll}\text { 3.3.2. Pressure gradient and hydraulic resistance } & 87\end{array}$

$\begin{array}{ll}\text { 3.4 Particle and solution characterisation } & 89\end{array}$

3.4.1 Vibrating Sample Magnetometry (VSM) 89

3.4.2 Superconducting Quantum Interference Device (SQUID) 90

3.4.3 UV-Visible spectrometry 93

$\begin{array}{ll}3.5 \text { Flow characterisation } & 96\end{array}$

3.5.1 Sample preparation $\quad 96$

3.5.2 Experimental protocol $\quad 97$

3.5.3 Observation methods 98

$\begin{array}{ll}\text { 3.6 Magnetic separation } & 99\end{array}$

3.6.1 Sample preparation $\quad 99$

3.6.2 Experimental protocol 101

3.6.3 Observation techniques 103

$\begin{array}{ll}\text { 4. RESULTS } & 107\end{array}$

$\begin{array}{ll}\text { 4.1 Properties of synthesised nanoparticles } & 107\end{array}$

4.1.1 Size and morphology 107

$\begin{array}{ll}\text { 4.1.2 Magnetic properties } & 109\end{array}$

$\begin{array}{ll}\text { 4.2 Properties of commercial microbeads } & 115\end{array}$

$\begin{array}{ll}\text { 4.2.1 Size and morphology } & 115\end{array}$

$\begin{array}{ll}\text { 4.2.2 Magnetic properties } & 117\end{array}$ 
$\begin{array}{ll}\text { 4.3.1 Channel reproduction } & 122\end{array}$

$\begin{array}{ll}\text { 4.3.2 Flow behaviour } & 125\end{array}$

$\begin{array}{lr}\text { 4.3.3 Mechanical strength } & 131\end{array}$

$\begin{array}{lr}\text { 4.3.4 Solvent compatibility } & 133\end{array}$

$\begin{array}{ll}\text { 4.3.5 Embedded magnet system } & 135\end{array}$

$\begin{array}{ll}\text { 4.3.6 Magnet-micrometer system } & 138\end{array}$

$\begin{array}{lr}\text { 4.3.7 Metglas system } & 140\end{array}$

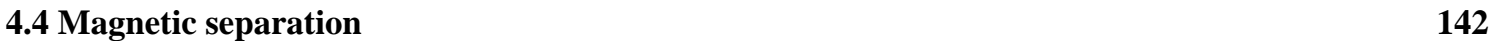

$\begin{array}{ll}\text { 4.4.1 Control } & 143\end{array}$

4.4.2 Basic separation $\quad 146$

4.4.3 Magnetic moment selection $\quad 151$

$\begin{array}{ll}\text { 4.4.4 Size selection } & 153\end{array}$

$\begin{array}{ll}\text { 4.4.5 Separation in viscous buffer solutions } & 155\end{array}$

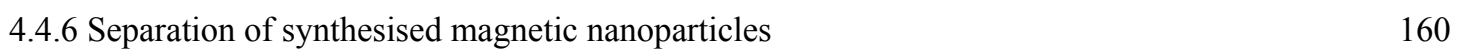

$\begin{array}{ll}\text { 4.4.7 Separation using Metglas system } & 162\end{array}$

5. DISCUSSION AND CONCLUSIONS 165

$\begin{array}{ll}\text { LIST OF SYMBOLS } & 173\end{array}$

$\begin{array}{ll}\text { REFERENCES } & 175\end{array}$ 


\section{Introduction}

Magnetic separation is a well-established technique, having been used in many industrial processes since the mid-1800s [1]. It essentially involves the use of a magnetic field gradient to isolate magnetic particles from nonmagnetic fluids or materials. The first magnetic separators used large electromagnets to extract iron from ferrous ores, and have been of great use in the minerals industry. The emergence of high gradient magnetic separation (HGMS) systems [2] over the last few decades has allowed magnetic separation to be used in more sensitive applications, including water treatment, chemical processing and biotechnology. However, as the size and/or concentration of magnetic particles decreases, increasingly large magnetic field gradients are needed for effective separation.

The use of microfluidic systems for magnetic separation is a relatively new field. Microfluidics refers to the manipulation of fluids in channels smaller than 1 millimetre in size [3]. The small fluid volumes involved bring many benefits for analytical applications, including reduced sample consumption, high sensitivity and shorter analysis times. Microfluidic channels also demonstrate unique flow properties which may be exploited in some applications. Additionally, multiple processes and detection systems can be integrated onto a single portable chip, often referred to as a Lab-on-achip (LOC) system. Finally, it is much easier to produce a strong magnetic field gradient over a small distance than a large one, meaning magnetic particles may be manipulated more readily in microfluidic channels. For these reasons microfluidic magnetic separation has developed rapidly in the last decade, and is already in use in a number of bioanalytical applications [4]. It should be noted that most biological samples are not strongly magnetic, and are therefore usually magnetically labelled by chemically binding magnetic microbeads or nanoparticles to their surface before separation takes place. This allows specific cell types to be isolated using a magnetic field, as the beads will only bind to those cells.

The primary aim of this project was to produce a microfluidic magnetic separation system suitable for the recovery of magnetic microbeads and nanoparticles. Microfluidic 
devices were produced in polydimethylsiloxane using a rapid prototyping process [5]. This technique is commonly used due to its low cost and quick turnaround time, allowing new designs to be readily produced. The system which was produced for this project employed an H-shaped channel layout. Fluid containing the particles was introduced into one inlet, while a buffer solution was flowed through the second. Laminar flow would prevent the two streams from mixing, but a magnetic field gradient could be applied across the middle channel to divert magnetic particles into the buffer stream.

This thesis describes the development and evaluation of this separation system. Background theory relevant to this work, including a discussion of the synthesis and properties of magnetic nanoparticles and microparticles, as well as an introduction to microfluidics, is presented in Chapter 2. That chapter also presents background specific to magnetic separation in microfluidic systems, including an analysis of the forces on a magnetic particle in a fluid, and a review of existing separation systems. The experimental procedures developed in the course of this project are described in Chapter 3 , with an emphasis on the fabrication process used to create the microfluidic devices used. Chapter 3 also provides details of the characterisation techniques used to evaluate the performance of the separation system. The experimental results, including characterisation of the magnetic particles, properties of the microfluidic devices and measurements of their ability to separate particles, are presented in Chapter 4. Finally, Chapter 5 provides discussion of the key results and conclusions. 


\section{Background theory}

In this section, theory relevant to the design and use of a microfluidic magnetic separation device is introduced. The properties of magnetic materials are discussed in Section 2.1, before explaining the unique magnetisation behaviour that emerges at the nanoscale. Magnetic nanoparticles and microbeads were both used in separation experiments for this project, and therefore synthesis techniques are outlined for both in Sections 2.2. An introduction to microfluidics is then presented in Section 2.3, covering fabrication techniques as well as the interesting flow properties demonstrated in microfluidic channels. This is followed by a description of the forces exerted on a magnetic particle in a fluid in Section 2.4, before ending with a review of current magnetic separation systems.

\subsection{Magnetic Materials}

The major focus of this project was the manipulation of magnetic particles, and it is therefore helpful to consider the properties of these materials. This information allows us to predict the motion of the particles in an applied field, as will be demonstrated in Section 2.4. While only ferromagnetic and superparamagnetic materials were used in this work, the simpler concepts of diamagnetism and paramagnetism will first be introduced.

It is useful to first consider the effect of an applied magnetic field in a vacuum. Magnetic flux will permeate through space as a result of an applied field $\vec{H}$. At a given region in space, the magnetic flux density $\vec{B}$ is given by

$$
\vec{B}=\mu_{0} \vec{H},
$$

where $\mu_{0}$ is the permeability of free space, which has a defined value of $4 \pi \times 10^{-7} \mathrm{H} / \mathrm{m}$. In a vacuum, or an environment containing only non-magnetic objects, the density of magnetic flux is dependent solely on the applied field. For example, applying a spatiallyuniform field will produce the spatially-uniform flux density pictured in Figure 1. 
The situation becomes more interesting if a magnetic material is introduced. The material will interact with the applied field, and affect the distribution of magnetic flux. The magnetic flux density is now represented by

$$
\vec{B}=\mu_{0}(\vec{M}+\vec{H}),
$$

where $\vec{M}$ is the magnetic moment per unit volume of the material, commonly referred to simply as the magnetisation. The magnetisation can be either negative or positive, depending on the form of magnetism the material displays. Materials with positive magnetisation will concentrate magnetic flux, while those with negative magnetisation will partially expel it. In the presence of a spatially-uniform applied magnetic field, the resulting flux density now varies with position, as shown in Figure 1.

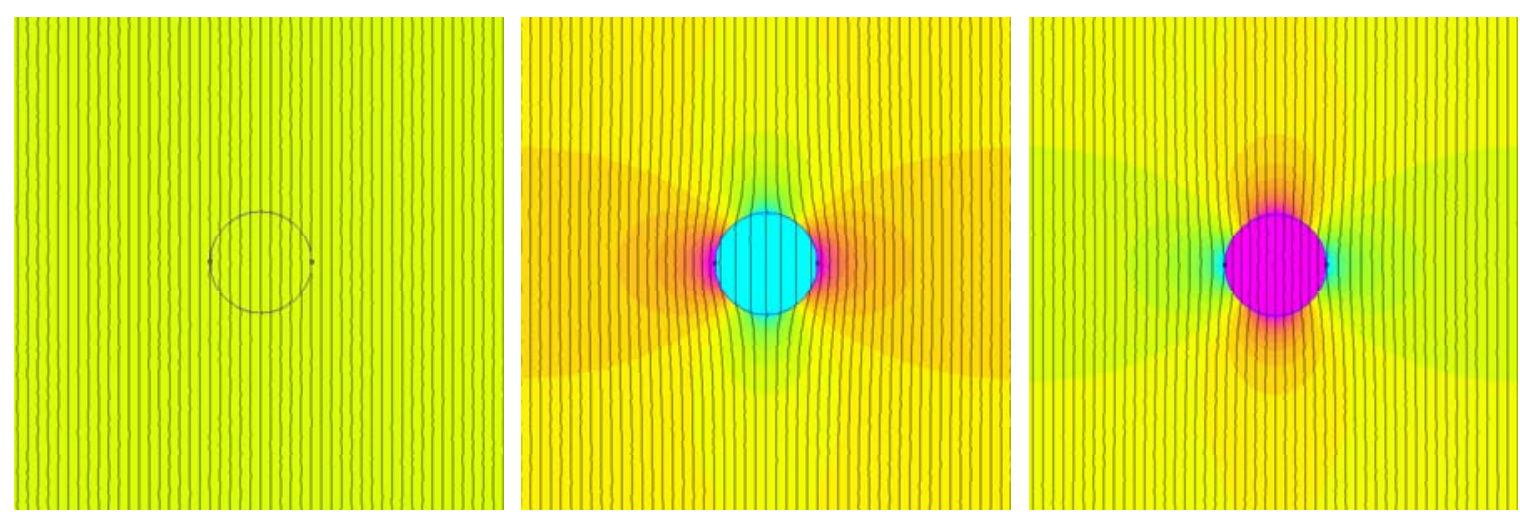

Figure 1: Magnetic flux density when a spatially-uniform magnetic field is applied vertically to a block of material with $M=0$ (left), $M<0$ (middle) and $M>0$ (right). Regions of high density are red, and regions of low density are blue. Diagrams produced using FEMM [6].

Regardless of the particular type of magnetism a material shows, the magnetisation $\vec{M}$ resulting from an applied field $\vec{H}$ may be represented by

$$
\vec{M}=\chi \vec{H},
$$

where $\chi$ is the volume magnetic susceptibility of the material. This treatment is particularly convenient when dealing with paramagnetic and diamagnetic materials, as $\chi$ is usually a scalar constant. For other forms of magnetism $\chi$ is a tensor, meaning its value will depend on both the current strength of the applied field and any previous field exposure. 


\subsubsection{Diamagnetism and paramagnetism}

The electron orbits in the atoms of any material constitute small localised currents, giving rise to small magnetic moments. In the absence of an external field these moments cancel, giving zero net magnetisation. However, the application of a magnetic field will strengthen the moments opposing the field, while weakening the moments aligned with the field. The resulting imbalance produces a net negative magnetisation, which scales linearly with the strength of the applied field. Diamagnetic materials are repelled by a magnetic field and will move toward regions of lower magnetic field strength. All materials exhibit diamagnetism, but because the effect is extremely small $(\alpha$ $\sim-10^{-5}$ ), it will be masked by any other form of magnetism the material may possess.

Paramagnetism occurs in materials containing unpaired electrons, with both the orbital motion and spin of the electrons contributing to the magnetic moment. Thermal effects will randomise these dipole moments, meaning in the absence of an applied field the net magnetisation is zero. However, an applied field will promote alignment of the dipoles, resulting in a net positive magnetisation. This alignment is continually disrupted by thermal fluctuations, meaning that the net effect is much smaller than the sum of all the

dipole moments $\left(\chi \sim 10^{-5}-10^{-2}\right.$ [7]). The intensity of the magnetisation may be modelled using the Langevin function [7]

$$
M=M_{s}\left[\operatorname{coth}\left(\frac{m H}{k T}\right)-\left(\frac{k T}{m H}\right)\right],
$$

where $M_{s}$ is the saturation magnetisation of the material (which results once all the dipole moments are aligned), $m$ is the dipole moment contributed by each unpaired electron, $k$ is Boltzmann's constant and $T$ is the sample temperature. Magnetic saturation may only be approached by applying extremely large fields at low temperature. Therefore, in most practical situations the magnetisation may be approximated as linear, in accordance with Curie's law [8]. Both models for paramagnetism are plotted in Figure 2. The models are consistent at low fields and/or high temperatures, but Curie's law clearly fails to account for the saturation of the material at higher fields. 


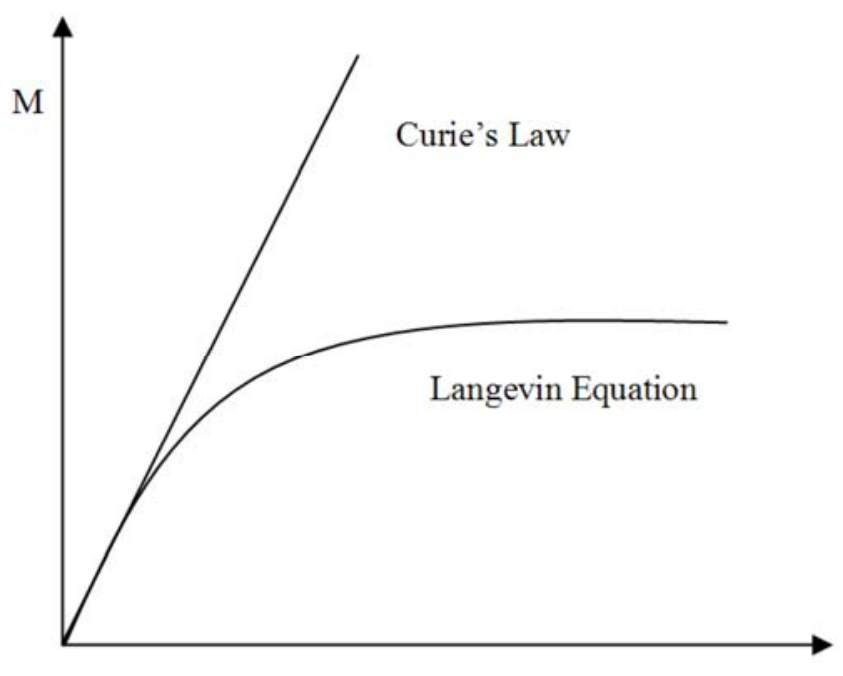

$\mathrm{H} / \mathrm{T}$

Figure 2: Comparison of the magnetisation curves for a paramagnetic material, as predicted using Curie's Law and the Langevin theory.

\subsubsection{Ferromagnetism and magnetic domains}

Ferromagnetic materials are distinct from paramagnetic and diamagnetic materials, as their magnetism persists even in the absence of an external magnetic field. This is because the dipole moments in the sample do not act independently. A brief introduction to the nature of the dipole interactions is provided in this section. Thorough reviews of the mechanism of ferromagnetism may be found in textbooks by Chikazumi [7], Cullity [9] or Kittel [10].

When two atoms are brought close together, the Pauli Exclusion Principle requires that the electrons do not occupy the same quantum state, which means that they must be described by an antisymmetric wavefunction. Therefore, if the electrons possess a symmetric spin function the wavefunction will be spatially antisymmetric, while an antisymmetric spin function will require a spatially symmetric wavefunction. The Coulomb repulsion between the electrons is (generally) minimised when the wavefunction is spatially antisymmetric, as the electrons are likely to be further apart [10]. This means that parallel electron spins are energetically favoured. This effect is referred to as the exchange interaction, and can produce very large magnetisations $(\chi \sim$ $10^{5}$ ). The associated quantum exchange energy $E_{e x}$ is given by

$$
E_{e x}=-J\left(\vec{S}_{i} \cdot \vec{S}_{j}\right),
$$


where $J$ is the exchange integral, and $\vec{S}_{i}$ and $\vec{S}_{j}$ are the electron spins. When $J$ is positive, the energy is minimised if the electron spins are parallel. The exchange energy is exceeded by thermal energies at what is known as the Curie temperature. Above this temperature the alignment between the spins is disrupted, and the sample becomes paramagnetic.

A ferromagnetic sample may minimise its exchange energy by aligning all its magnetic moments in one direction, magnetically saturating the material. However, this will produce a large magnetic field outside of the sample, incurring an energy cost called the magnetostatic energy. Competition between minimising the exchange and magnetostatic energies leads to the formation of magnetic domains, where the magnetic moments are aligned within a domain, but not between adjacent domains. Figure 3 illustrates how forming these domains reduces the amount of stray field outside the material.
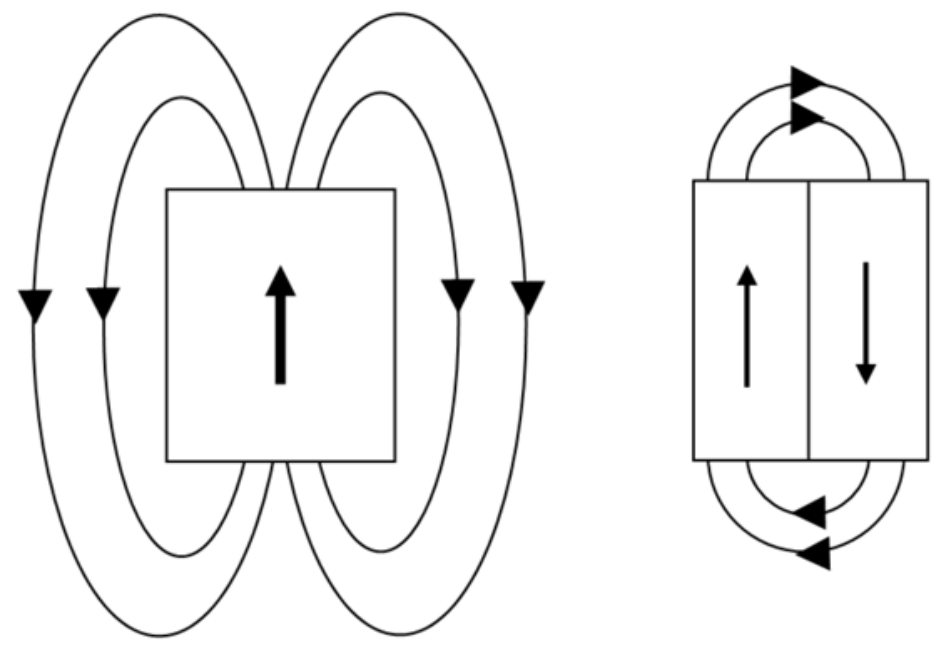

Figure 3: Comparison of the stray field outside a ferromagnetic material when partitioned into one domain (left) and two domains (right). The multi-domain sample has less flux outside the sample, and hence a lower magnetostatic energy.

Creating $\mathrm{N}$ domains approximately reduces the magnetostatic energy by a factor of $1 / \mathrm{N}$, meaning an infinite number of domains would minimise this contribution. However, the actual number realised is limited by the domain wall energy. If the interface between two domains was abrupt, a large energy cost would result from forcing adjacent electron spins into antiparallel alignment. It is energetically favourable to instead transition gradually from one domain to the next, creating what is known as a Bloch zone. The 
width of this zone is typically around 100 times the material's lattice constant [11]. For pure iron, which has a lattice constant of $0.29 \mathrm{~nm}$, domain walls would be roughly 29 $\mathrm{nm}$ wide. It should be noted that there is still an energy cost associated with the formation of a Bloch zone, as it necessitates the loss of spin alignment with the easy magnetisation directions of the material.

The behaviour of magnetic domains in an applied field leads to complicated magnetisation behaviour. The state of magnetisation is no longer dependent on just the current applied field, but will also exhibit a memory effect. Figure 4 shows the magnetisation curve of a typical ferromagnetic material, which may be understood by considering the changes in the domain structure as the field is changed. The material initially has no net magnetisation, as the magnetic moment of domains aligned in one direction will be cancelled by those aligned in the opposite direction. When a magnetic field is applied, domains which are parallel to the field will grow at the expense of the remaining domains, and a net magnetisation is obtained. At high enough fields, the parallel domains will fill the whole sample, and the saturation magnetisation $M_{s}$ is reached. If the field is then removed, the original magnetisation state is once again the lowest energy state. However, domain walls become pinned to defects and grain boundaries in the material, preventing complete recovery of the starting domain configuration. A stable remanence magnetisation $M_{r}$ is instead attained. Applying a field in the opposite direction allows pinning to be overcome, and the magnetisation is brought back to zero. The field needed to achieve this is called the coercive field $H_{c}$. 


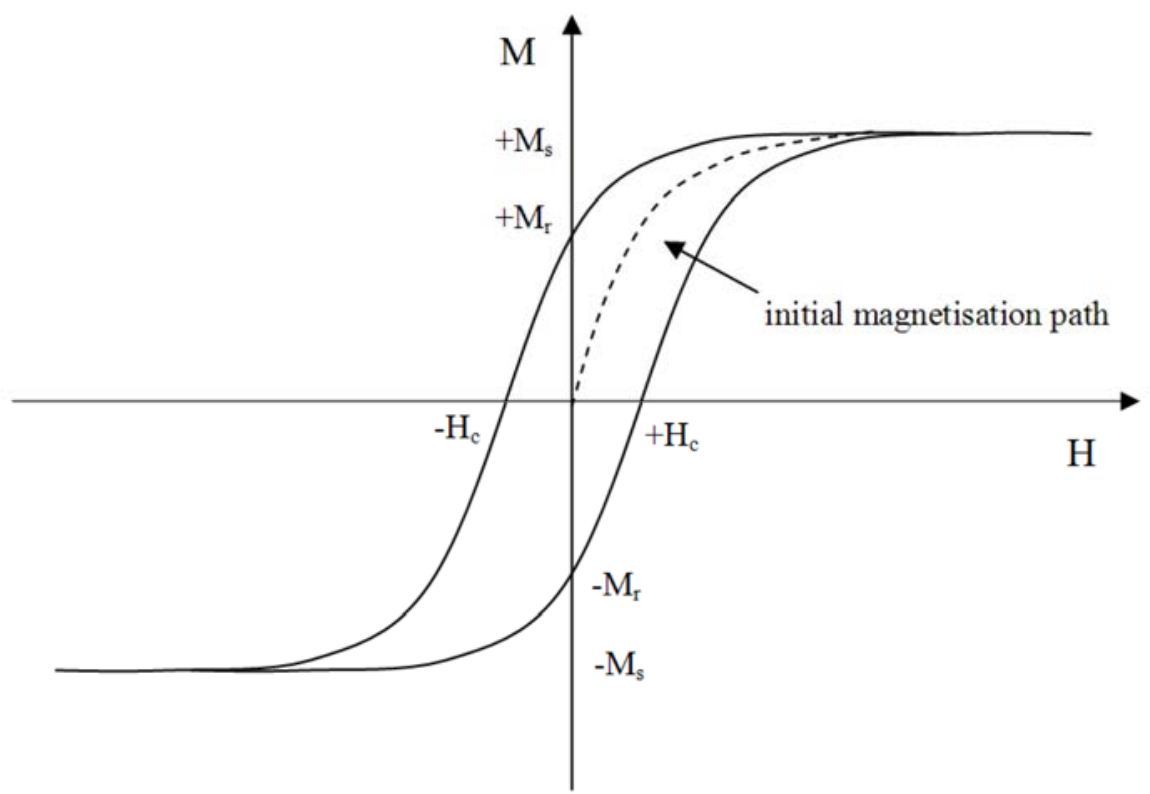

Figure 4: Magnetisation curve for a ferromagnetic material. The coercivity $H_{c}$, remanence $M_{r}$ and saturation $M_{s}$ are indicated on the curve.

\subsubsection{Magnetic anisotropy}

In most cases it is found that it takes smaller fields to saturate a magnetic material in certain preferred directions. These directions are referred to as the easy axes of the material. Hard axes refer to the directions in which saturation requires larger fields. The extra energy required is the product of the anisotropy energy density $K$ and the sample volume $V$. This anisotropy may be brought about by several independent effects, some of the most important of which are introduced below.

Magnetocrystalline anisotropy is important to the magnetisation of any crystalline material. The energy of an electron will depend on the orientation of the occupied orbital with respect to the crystal lattice. Interaction between the spin and orbital magnetic moments, known as spin-orbit coupling, will then mean that it will cost more energy to align the electron spins along particular crystal axes. Magnetisation is a result of the alignment of electron spins, meaning that it will be easier to magnetise a sample along certain crystallographic axes than others. For example, it is easier to magnetise bulk iron along the edge of its unit cell than through the body or face diagonals, as shown in Figure 5 [12]. The magnitude of the saturation magnetisation is independent of direction, but a larger field is required to reach it along the hard axis. 

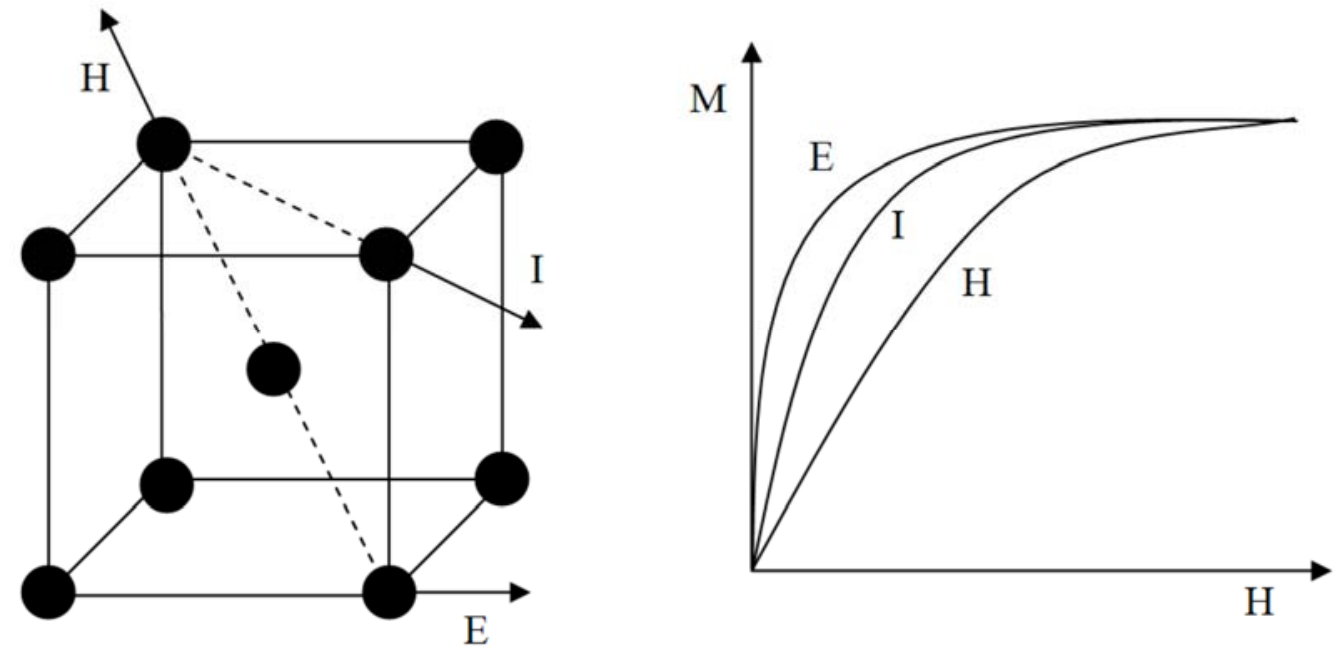

Figure 5: Easy (E), intermediate (I) and hard (H) axes of magnetisation for iron. Schematic magnetisation curves for each axis are also shown.

Shape anisotropy is not a property of the material itself, but rather the dimensions of the sample. When an external field is applied to the sample, a net current is formed at the surface. This current produces a demagnetising field [7] which partially cancels the applied field, meaning larger fields are required to saturate the material than would otherwise be needed. The strength of the demagnetising field decreases as the poles are moved further apart, making it easier to magnetise a material along its length than across it. Figure 6 illustrates this for a rectangular sample. Although the effect is typically associated with the shape of a macroscopic sample, shape anisotropy is equally important at the nanoscale. Magnetic nanoparticles synthesised in this project were nominally spherical in shape, but any deviations from this shape would lead to a preferred magnetisation direction for each particle. We would however expect this contribution to the anisotropy to be relatively small, as even if the particles are not perfectly spherical, their aspect ratio should still be close to 1 . 

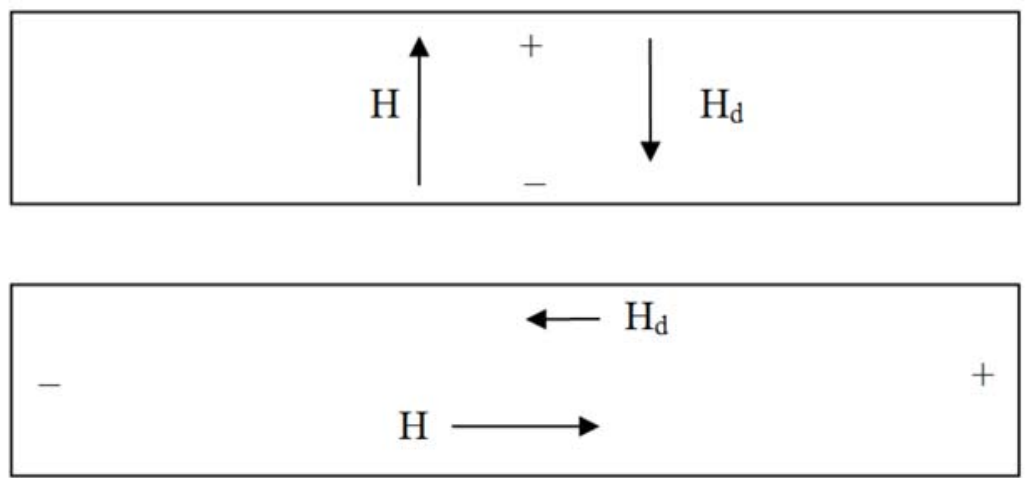

Figure 6: Illustration of shape anisotropy in a magnetic material. When an external field is applied across the sample width (top), the field is largely cancelled by the demagnetising field. The same field is cancelled to a lesser degree when it is instead applied along the sample length (bottom).

Surface anisotropy is of particular importance to nanostructures. This form of anisotropy exists due to the termination of the crystal lattice at the boundaries of the sample [13]. Disorder and vacancies at this interface produce local fields which will favour a certain spin alignment direction. As illustrated in Figure 7, this alignment will be either normal or tangential to the surface, depending on whether the anisotropy is positive or negative. For a macroscopic sample this contribution is much smaller than the magnetocrystalline anisotropy. However, as the particle volume is decreased down into the nanoscale, surface anisotropy becomes increasingly important.
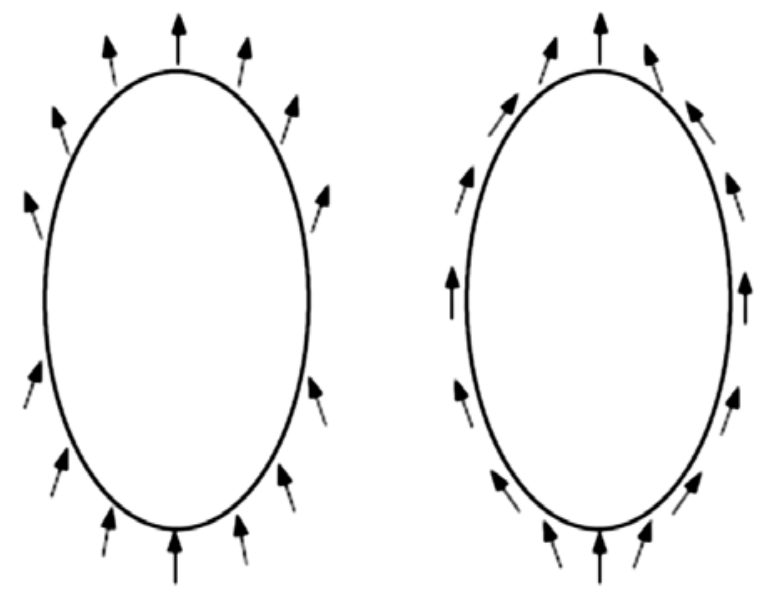

Figure 7: Surface spin arrangement of a ferromagnetic particle with $K<0$ (left) and $K>0$ (right). Reproduced from [13]. 


\subsubsection{Monodomain and superparamagnetic materials}

If a ferromagnetic sample is smaller than the width of a domain wall, domain creation generally becomes energetically unfavourable. The sample instead becomes a single domain, with all the atomic moments aligned in one direction. Monodomain particles have been experimentally observed, for example by Majetich and Jin [14]. It is interesting to note that in some cases, particles significantly larger than the width of a domain wall can still be monodomain. For example, MnBi has a domain wall width of $\sim 10 \mathrm{~nm}$, but remains monodomain up to a particle size of $\sim 500 \mathrm{~nm}$ [11]. The ability of $\mathrm{MnBi}$ to remain monodomain is a result of its strong magnetocrystalline anisotropy, which makes the energy cost of forming domain walls comparatively high. By contrast, Fe, which has a much lower anisotropy, is expected to form domains at particle sizes above $\sim 15 \mathrm{~nm}$. In general the maximum monodomain particle diameter is given by [9]

$$
d=\lambda\left(\frac{E_{\text {anisotropy }}}{E_{\text {dipole }}}\right)^{n},
$$

where $\lambda$ is the domain wall width, while $E_{\text {anisotropy }}$ and $E_{\text {dipole }}$ are the anisotropy and magnetostatic energies of the sample respectively. The exponent $n$ is equal to $1 / 2$ for lowanisotropy materials and 1 for high-anisotropy materials. If we only consider the magnetocrystalline contribution to the anisotropy, we can use Equation (6) to predict that iron and magnetite particles will be monodomain up to diameters of 14 and $128 \mathrm{~nm}$ respectively. The difference in critical diameter is mostly due to the higher magnetocrystalline anisotropy of the oxide.

Monodomain particles are usually considered as being magnetised to saturation in one direction or the opposite direction. All the atomic moments are aligned through the exchange interaction. Changing the magnetisation direction therefore occurs through coherent rotation of the moments, as opposed to the growth and shrinking of domains seen in macroscopic samples. If it is assumed that the anisotropy is uniaxial, the associated energy barrier can be simplistically described by

$$
E=K V \sin ^{2} \theta-\mu H \cos \theta,
$$

where $V$ is the particle volume, $K$ is the magnetocrystalline anisotropy energy density, $\mu$ is the existing magnetic moment, and $H$ is the applied field. The angle $\theta$ is taken 
between the original magnetisation direction and the applied field direction. A few key points may be taken from this equation. Firstly, applying a magnetic field reduces the height of the energy barrier, with stronger fields naturally being more effective at helping to surmount the energy gap. Secondly, the energy required to change the magnetisation decreases with decreasing particle size, meaning lower applied fields are needed. This is equivalent to a decrease in the coercivity of the sample.

Should the particle become small enough, the energy barrier will become comparable to thermal energies, even at room temperature. At this point the magnetisation of the particle will fluctuate randomly, and it is said to be superparamagnetic. The atomic moments of the crystallite will remain aligned with one another, as the exchange energy is still much larger than the thermal energy. The direction of all the moments in the crystallite will fluctuate in step with one another.

To predict whether particles will behave superparamagnetically, we need to consider the time needed for the magnetisation direction of a monodomain particle to be flipped by thermal fluctuations. This is known as the Neel relaxation time, which is described by the Arrhenius law [15]

$$
\tau=\tau_{0} e^{\frac{\Delta E}{k T}} \approx \tau_{0} e^{\frac{K V}{k T}}
$$

where $\Delta E$ is the energy required to flip the magnetisation, and $\tau_{0}$ is the "attempt time" of the material, which is typically taken as $10^{-9} \mathrm{~s}$, although some studies have favoured values as low as $10^{-12} \mathrm{~s}[16]$. Here we assume the barrier takes the form of Equation (7), meaning $\Delta E$ is equal to the magnetocrystalline anisotropy energy $K V$. If the relaxation time is shorter than the time needed to measure the magnetisation (conventionally taken to be 100 seconds [17]) then the particle is considered to be superparamagnetic. From Equation (8), spherical particles of pure iron $\left(K=1.2 \times 10^{4} \mathrm{Jm}^{-3}[14]\right)$ would be expected to show room-temperature superparamagnetism at diameters less than $14 \mathrm{~nm}$. For a highly anisotropic material such as iron carbide $\left(K=9.8 \times 10^{5} \mathrm{Jm}^{-3}[18]\right)$ the diameter shrinks to $7 \mathrm{~nm}$. The Arrhenius Law is intended only as a guide, and it is possible for particles to exhibit superparamagnetism at sizes well above the critical diameter. 
A monodomain or superparamagnetic particle is always magnetically saturated in some direction, even in zero applied field. However in practice, measurements observe the net magnetisation of a large number of particles, giving a different magnetisation curve. The magnetisations of individual particles are randomly orientated, leading to zero net magnetisation. Once an external field is applied however, the moments of the particles will tend to align with the field, and the net magnetisation will increase. At very high fields, all the moments will align, and magnetic saturation is reached. Subsequently removing the field will cause all the moments to lose coherence again, as there is no energy barrier present to prevent it. The resulting magnetisation curve is shown in Figure 8. The curve is reminiscent of that of a paramagnetic material, as was seen in Figure 2, and this is not coincidental. Paramagnetism involves the alignment of an ensemble of independent magnetic dipoles, which would otherwise be randomly oriented by thermal fluctuations. Superparamagnetism shares the same mechanism, except the weak atomic moments are replaced with the moments of entire particles. Consequently superparamagnetic materials feature the zero coercivity and remanence seen in paramagnetic materials, but their saturation magnetisation is comparable to ferromagnetic materials.

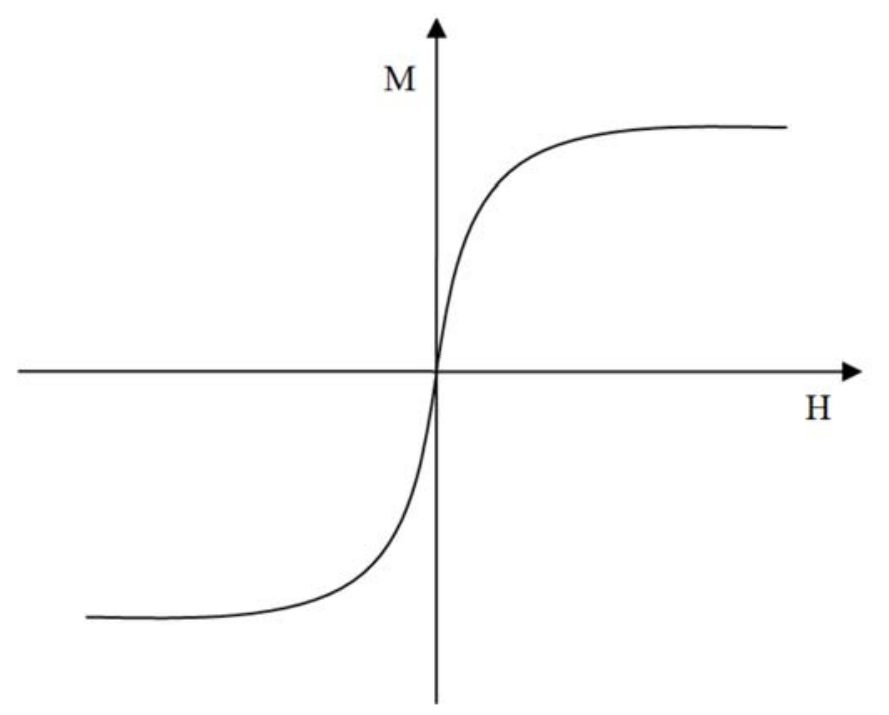

Figure 8: Magnetisation curve for a superparamagnetic material. The remanence and coercivity are both zero, similar to paramagnetic materials.

The magnetisation of a superparamagnetic material in an applied field $H$ may be described by a Langevin equation 


$$
M=M_{s}\left[\operatorname{coth}(\alpha H)-\frac{1}{\alpha H}\right]
$$

where $M_{s}$ is the saturation magnetisation. The value $\alpha$ is a fitting parameter equal to

$$
\alpha=\frac{\mu_{0} M_{s} V_{p}}{k T},
$$

where $V_{p}$ is the volume of each nanoparticle. This treatment is similar to the Langevin theory of paramagnetism, except that the individual dipole moments are replaced with the moments of the nanoparticles.

\subsection{Magnetic nanoparticles and microbeads}

Particles used in this project for separation experiments were magnetic nanoparticles, usually encapsulated in a polymer to form magnetic microbeads. In this section, common techniques for the synthesis of magnetic nanoparticles and microbeads will be reviewed. Key applications of these particles will also be discussed. Due to the depth of this area of research, the scope of this review will be limited to iron-based particles obtained through solution-phase synthesis. Extensive reviews of the synthesis and applications of magnetic nanoparticles are presented by Lu et al. [19], Teja et al. [20], Tartaj et al. [21,22], Cushing et al. [23] and Pankhurst et al. [24].

\subsubsection{Synthesis of magnetic nanoparticles}

The preparation of monodisperse magnetic nanoparticles in solution is usually achieved through controlled precipitation reactions. The concentration of reactants is gradually increased until the solution is supersaturated, at which point particles will begin to nucleate. The reactant concentration will then gradually decrease as monomers in the solution diffuse to the surface of the particles and feed their growth. Ideally nucleation should only occur in a short burst at the start of the reaction, and be avoided during the particle growth period. This simple growth model was proposed by LaMer and Dinegar [25], and is represented by curve I in Figure 9. If nucleation events continue during the growth period, a wide range of nanoparticle sizes may be produced. 


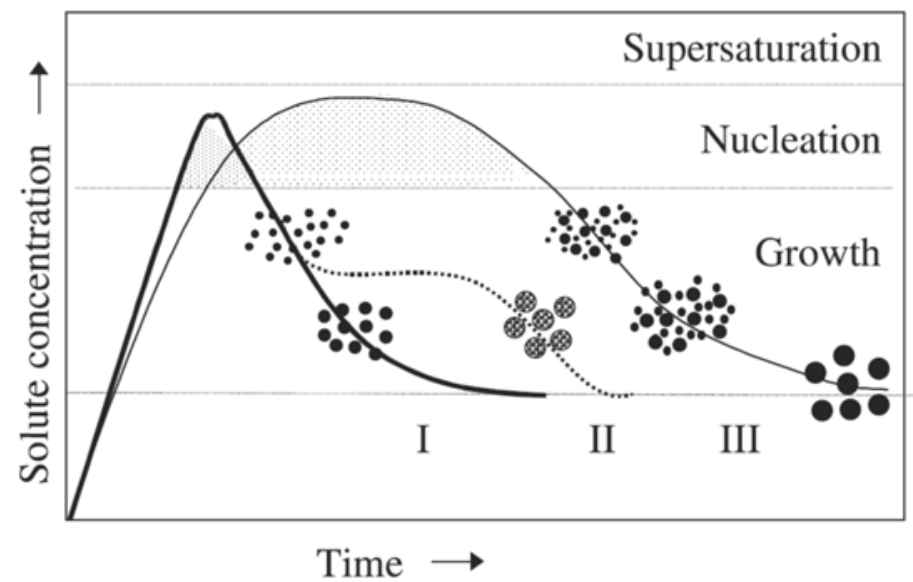

Figure 9: Suggested mechanisms for solution-phase nanoparticle growth. Curve I: Independent nucleation and growth. Curve II: Growth by aggregation of smaller particles. Curve III: Ostwald ripening growth. Image reproduced from [21].

Monodisperse nanoparticles are sometimes still obtained following multiple nucleation events, prompting the development of alternative growth mechanisms. Ouden and Thompson [26] noted that in cases where the nucleation rate is much greater than the growth rate, as represented in Curve III of Figure 9, particles of uniform size are often obtained. In this case small particles, which have a high surface energy, are absorbed by larger ones. This process, referred to as Ostwald ripening, means that particles that nucleate during the growth period contribute to the growth of existing particles. Particle growth can also be driven by the aggregation of 'primary' particles of equal size, as represented in Curve II of Figure 9. Diffusion of monomers to the surface of these aggregates will then sustain particle growth [27].

The most popular synthesis technique for producing iron oxide nanoparticles is coprecipitation, in which nucleation is artificially triggered before supersaturation is reached. Spherical magnetic particles $30-100 \mathrm{~nm}$ in diameter can be obtained by reacting a Fe(II) salt with a base and a mild oxidant [28]. Ageing a mixture of ferrous and ferric hydroxides will also produce magnetite or maghemite particles 2-15 nm in size [22]. The size of the nanoparticles produced is strongly dependent on a number of factors, including the concentration of cations, the nature of the counterions and the $\mathrm{pH}$ of the solution [20]. Both methods are carried out in aqueous solution, making them ideal for biomedical applications. 
The large surface-area to volume ratio of nanoparticles means they will often aggregate to reduce their surface energy [29]. Surfactants are therefore usually added to the surface of the nanoparticles to help maintain a stable dispersion. Anionic surfactants have been shown to prevent aggregation by increasing the electrostatic repulsion between particles $[30,31]$. However, the stability of the suspension is very sensitive to conditions such as $\mathrm{pH}$ and ionic strength. Stabilisation can be more conveniently achieved using neutral surfactants such as starches [32], proteins [33] and polyelectrolytes [31]. In particular, oleic acid is commonly used to stabilise magnetite particles [34].

Thermal decomposition of organometallic precursors in organic solvents has been shown to produce magnetic nanoparticles with excellent size control. Particles produced through this method also show a high degree of crystallinity, which is associated with the high reaction temperatures used. Nanoparticles have been produced using a variety of precursors, including iron acetylacetonates [35], iron carboxylates [36] and iron carbonyls [37]. Organic surfactants, generally oleic acid [35] or oleylamine [38], are required to stabilise the particles and control the rate of growth. The size and shape of the resulting particles is crucially dependent on the ratio of starting reagents, and mildly dependent on the reaction temperature and time [19]. A protocol developed by Cheong [39] was used to produce iron oxide, iron and iron carbide particles for use in this project. The details of this method are presented in Section 3.2.

\subsubsection{Synthesis of magnetic microbeads}

As discussed in Section 2.1.4, magnetic nanoparticles are often superparamagnetic, which means they should not agglomerate in the absence of an applied field. This property is useful in many in vitro biomedical applications. However, the high density of magnetic nanoparticles can make them prone to sedimentation, and their surfaces are difficult to functionalise. A common workaround is to disperse magnetic nanoparticles inside polymer beads. Organic polymers provide more flexibility for surface modification, and have a density similar to that of water [40]. The superparamagnetic behaviour of the nanoparticles is also preserved. The main drawback of using magnetic microbeads is that the effective particle magnetisation is reduced, depending on the amount of magnetic material present in the beads. 
Magnetic microbeads can be produced using a variety of methods. Zaitsev et al. [41] produced beads $50 \mathrm{~nm}$ in diameter by reacting methacrylic acid and hydroxyethyl methacrylate in the presence of magnetite nanoparticles. The magnetite particles were bound in the polymer as it formed, and were therefore evenly distributed throughout the beads. Alternatively, magnetite particles can be dispersed through a polymer solution, before inducing cross-linking of the polymer [42,43]. Considerable energy must be supplied to adequately disperse the particles in the viscous monomer solution, but the resulting beads show excellent chemical resistance and often enhanced magnetic content [44].

A drawback of the above synthesis methods is that the resulting beads are typically nonspherical, with a high degree of surface roughness. Spherical magnetic beads are usually prepared using monodisperse polystyrene beads, either by incorporating magnetic nanoparticles into the pores of the beads [45] or by depositing them on the surface [44]. The beads are then coated with a thinner second layer of polystyrene to seal in the nanoparticles. This is the approach used in the manufacture of commercial Dynabead and Spherotech magnetic beads. The scanning electron microscope image displayed in Figure 10 shows that spherical particles with randomly-distributed magnetic content can be obtained. Beads produced using these techniques are generally $1 \mu \mathrm{m}$ or greater in size. It is important to note that the polystyrene surface renders the beads hydrophobic, making surface functionalisation necessary in order to allow dispersion in water [46].

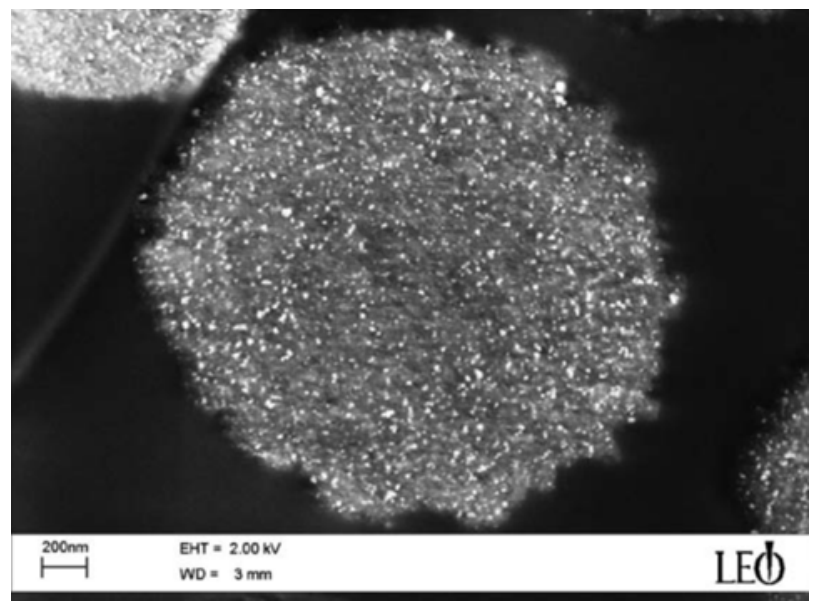

Figure 10: SEM image of a $2.8 \mu \mathrm{m}$ diameter Dynabead. Bright spots indicate the presence of iron oxide nanoparticles. Image reproduced from [45]. 


\subsubsection{Applications of magnetic nanoparticles and microbeads}

Magnetic nanoparticles have a large number of established and potential applications. Iron oxide nanoparticles have attracted significant interest, as they exhibit good magnetic properties and are relatively inexpensive to produce. Additionally their stability and low toxicity make them suitable for use in biomedical applications.

Iron oxide particles have been used in recording and data storage applications for several decades [47]. In general a substrate is coated with a dispersion of the particles in an organic binder. The magnetisation state of individual particles or clusters may then be changed or read using an external probe, such as the recording and playback heads of a video cassette recorder. It is important that the magnetisation is stable in order to prevent data loss, meaning that the material used should have a reasonably high coercivity. For this reason, the iron oxide particles are often coated or doped with low levels of cobalt to increase the coercivity of the material [20]. Increasing storage capacity can be achieved by maximising the bit density of the storage medium. Bit density describes the amount of data that may be stored in a given area, and can be maximised by reducing the size of the magnetic particles. The development of iron oxide nanoparticles of uniform size has therefore been important to the improvement of storage devices. Additionally, iron oxide nanoparticles in a nonmagnetic matrix undergo substantial changes in electrical resistance when exposed to small magnetic fields. This effect, known as giant magnetoresistance, has been exploited in the manufacture of ultra-sensitive recording heads and magnetometers $[11,48]$.

The use of iron oxide nanoparticles in ferrofluids has been extensively developed for both commercial and academic applications. Ferrofluids contain a high concentration of superparamagnetic nanoparticles, stabilised by a surfactant [49]. The particles remain dispersed even when magnetic field is applied, making the solution behave like a magnetic fluid. This behaviour can be clearly seen in Figure 11, in which a droplet of ferrofluid is placed near the pole of a bar magnet. The fluid conforms to the flux lines of the magnet, producing a spiked pattern. The ability to trap ferrofluids using a magnetic field means they are frequently used in magnetically-actuated seals, for example in computer hard disk drives [50]. Ferrofluids are also commonly used to dissipate heat in 
loudspeakers, by exploiting the decrease in magnetic susceptibility of the ferrofluid with increasing temperature. A magnet is used to draw cold fluid towards the speaker coil, thereby displacing warm fluid towards a heatsink. Applications of ferrofluids currently under development include magnetically deformable mirrors [51] and tools for eye surgery [52].

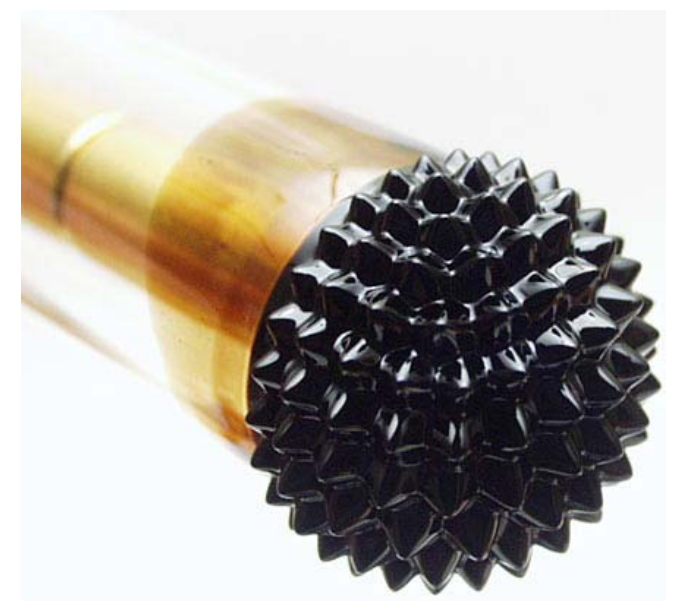

Figure 11: A droplet of ferrofluid near the pole of a permanent magnet [53]. The fluid interacts with the magnetic field, altering both the shape of the droplet and the magnetic flux distribution.

The use of magnetic microbeads for in vitro applications is fairly well developed. A number of manufacturers provide beads and separation systems for cell separation $[54,55]$. These systems consist of magnetic microbeads with surfaces functionalised to bind chemically to a particular target cell type, and a magnetic column to extract the beads. These systems have been used to enrich proteins [56], as well as to remove contaminants from water [57]. The superparamagnetic nature of the beads is crucial to the success of the process, as it is necessary to redisperse the beads in solution following their extraction. If the beads were ferromagnetic, they would remain agglomerated even after the magnetic field was removed.

Magnetic nanoparticles also show great promise in a number of in vivo applications. Here the small size of the particles is important, as it enables transport through the tissues and organs of the body without the risk of forming blockages in blood vessels [24]. Superparamagnetic particles are preferred, as they minimise the risk of particles forming large clusters in the body. 
Magnetite nanoparticles have been used as contrast agents for magnetic resonance imaging (MRI) [58,59]. They are referred to as $\mathrm{T}_{2}$ contrast agents, because they generate variations in the local magnetic field that reduce the $T_{2}$ (spin-spin) relaxation time of water molecules in the surrounding tissue. This reduction in $\mathrm{T}_{2}$ leads to dark spots in MRI images, allowing one to identify where the nanoparticles have accumulated. Currently most MRI contrast agents rely on passive targeting, in which the nanoparticles are taken up more readily by tumour cells than healthy cells due to differences in their composition [60]. Sensitivity may be further improved through active targeting, in which the nanoparticle surface is functionalised to selectively bind to the target cell [61].

In addition to diagnosis, magnetic nanoparticles have the potential to be used in the treatment of certain medical conditions. Magnetic nanoparticles could be used to transport drugs to specific regions of the body, as selected by an applied magnetic field [62]. This would allow the total dosage of a drug to be minimised, reducing damage to healthy tissues. Alternatively, nanoparticles could be used to generate localised heating in an AC magnetic field, thus destroying tumour cells through hyperthermia [63]. Heat is

produced through energy losses during the rotation of the magnetisation within the nanoparticles. Nanoparticles are capable of producing far more heat than microparticles through this process, because their magnetisation changes entirely through moment rotation rather than domain wall movement [64].

\subsection{Microfluidics}

Microfluidics is the study of the controlled flow of liquids or suspensions in submillimetre-sized channels [4]. At this scale, interesting flow properties are exhibited and may be exploited. Although basic theory has been understood for decades, interest in the development of microfluidic devices has increased dramatically over the last 15 years, due to the emergence of lab-on-a-chip (LOC) systems as a new field of research. These systems aim to integrate multiple laboratory-scale processes and detectors onto a single silicon or polymer chip, and show promise for applications in biomedicine and other fields. The extensive library of microfabrication techniques developed by the microelectronics industry has also facilitated the development of silicon and glass-based LOC systems. More recently, the introduction of various polymer-based fabrication 
techniques has reduced fabrication time and costs, allowing more laboratories to conduct research into microfluidics.

In this section, key advantages and applications of the aforementioned LOC systems will be summarised. Two important properties of the fluid flow in a microchannel, laminar flow and diffusive mixing, will then be introduced. Finally, common techniques to fabricate microfluidic devices and methods of regulating fluid flow will be discussed.

\subsubsection{Lab-on-a-chip systems}

Lab-on-a-chip (LOC) systems attempt to integrate miniaturised fluid channels and detection systems onto a silicon, glass or polymer chip. They are the fluidic analogue to micro-electromechanical systems (MEMS), which feature miniaturised electrical and mechanical components on a single chip [65]. Miniaturisation of laboratory-scale processes onto a microfluidic chip carries several advantages. The solution volume required is greatly reduced, as the volume of a microchannel is orders of magnitude less than that of a test tube. Volumes as low as $10^{-18}$ litres may be handled [3]. This is of particular value to biomedical research, where it may be very costly or impossible to produce reagents in large quantities. Smaller volumes are also associated with faster reaction times and more efficient detection schemes [4].

LOC systems are expected to allow complicated testing to be easily conducted during field-work. The devices are highly portable, allowing testing to be performed on-site, rather than transporting samples back to a lab. This would be highly useful for applications in remote locations, or where samples may degrade if not immediately tested. Process sequences may be fully automated, allowing non-experts to carry out testing. Finally, much like microelectronic systems, LOC systems may be cheaply massproduced. This means that it is generally affordable to use chips on a disposable basis.

Much of the early research into LOC systems in the 1990s was driven by the United States Defence Advanced Research Projects Agency (DARPA) [3]. Programmes focused on the development of chips that could be used to test for chemical and biological agents. The unique advantages offered by microfluidics (high sensitivity, fast reaction times, portability and low cost) make the technology ideal for such an 
application. The rapid growth of molecular biology since the 1980s also provided demand for LOC devices for DNA sequencing [66], cell culture [67,68] and other applications. Since then potential applications have diversified, to include the manipulation of magnetic materials [69] and the formation of monodisperse bubbles and droplets [70].

\subsubsection{Laminar flow}

Under normal conditions, fluid flow through a microfluidic channel is laminar, meaning no turbulence is present. To understand why this is the case, consider the flow of an incompressible fluid, which is described by the Navier-Stokes equation [49]

$$
\rho\left(\frac{d \vec{v}}{d t}+\vec{v} \cdot \nabla \vec{v}\right)=-\nabla p+\eta \nabla^{2} \vec{v}+\vec{f},
$$

where $v$ is the flow velocity, $\rho$ is the fluid density, and $\eta$ is the fluid viscosity. The lefthand side of Equation (11) represents the change in momentum experienced by an elemental fluid volume. The right-hand side of the equation states that this change in momentum arises from the pressure drop ( $1^{\text {st }}$ term) as well as the frictional forces on the liquid $\left(2^{\text {nd }}\right.$ term). The $3^{\text {rd }}$ term represents any remaining forces on the fluid, such as gravitational or centrifugal forces, which are often sufficiently small to neglect.

In 1883 Reynolds [4] noted the importance of the ratio between the kinetic energy stored in the fluid to the energy lost through friction. This ratio simplifies to what is now referred to as the Reynolds number

$$
\operatorname{Re}=\frac{\rho v L}{\eta}
$$

where $L$ is the smallest length scale of the channel. The Navier-Stokes equation may then be rewritten without dimensions as

$$
\frac{d \vec{v}}{d t}+\vec{v} \cdot \nabla \vec{v}=-\nabla p+\frac{1}{\operatorname{Re}} \nabla^{2} \vec{v}+\vec{f}
$$

When Re is very large, the frictional term becomes insignificant, indicating that the inertia of the fluid will dominate. Small eddy currents will grow unchecked, and turbulent flow will be observed. When the number is small however, viscous effects begin to dominate, and damp any eddy currents before they can grow. Under these 
conditions the flow is laminar. The differences between turbulent and laminar flow are illustrated in Figure 12. In the laminar flow regime, the fluid moves in parallel layers which do not mix. The pattern of flow lines will not vary with time once it has been established. By contrast, turbulent flow features chaotic swirling flow patterns, and the overall flow pattern will vary significantly with time.

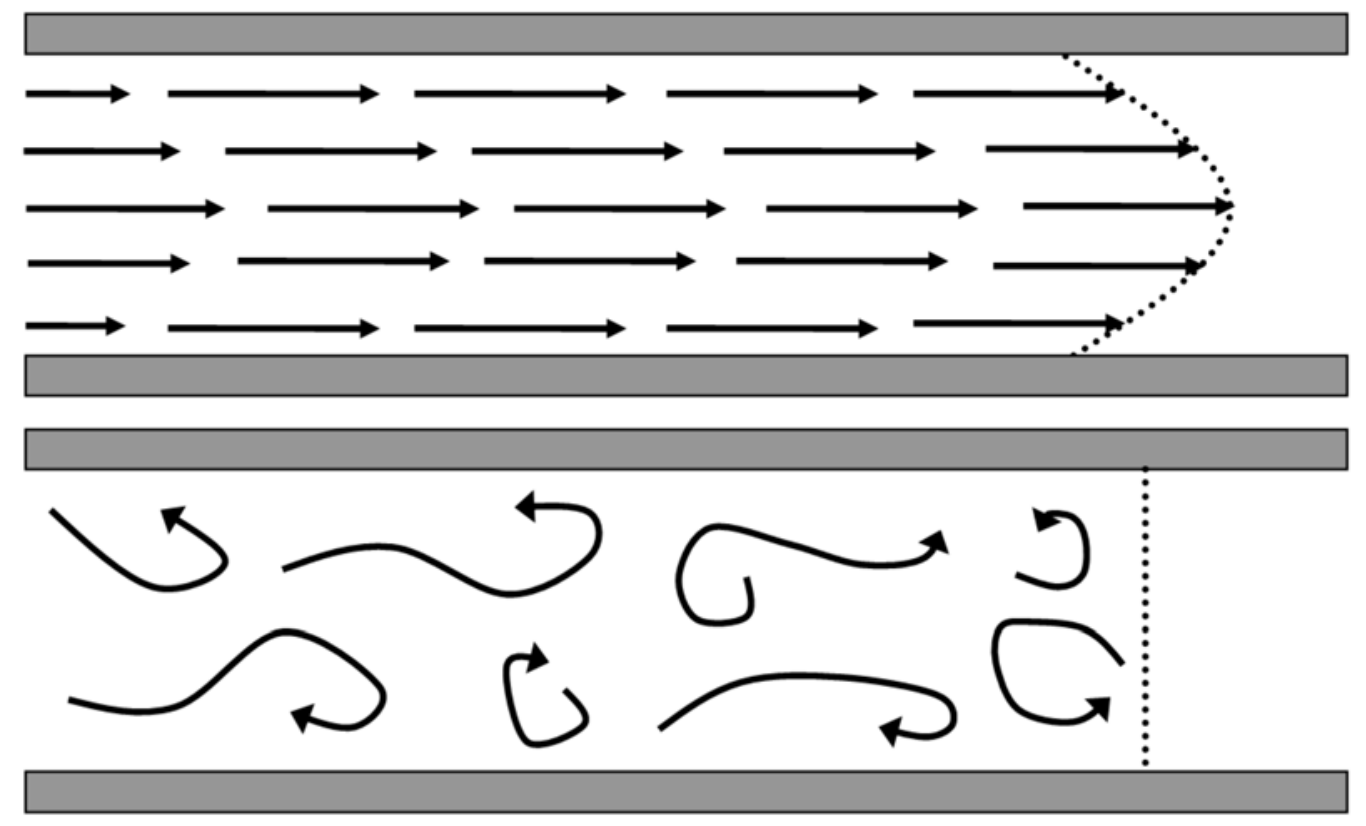

Figure 12: Schematic of fluid flow in a straight pipe when the flow is laminar (top) and turbulent (bottom). The dotted line indicates the position of the fluid front.

The Reynolds number allows us to predict the nature of the flow in a channel. For a straight pipe, laminar flow will dominate when Re is less than 2300. The flow becomes completely turbulent by the time Re exceeds 4300 [71]. For intermediate values of Re the flow is in a transition regime, displaying both laminar and turbulent flow behaviour. For a microfluidic channel of height $150 \mu \mathrm{m}$, supporting a flow of water $(\rho=0.998$ $\mathrm{g} / \mathrm{cm}^{3}, \eta=1 \mathrm{mPa} \cdot \mathrm{s}$ ) at a flow velocity of $2 \mathrm{~mm} / \mathrm{s}$, the Reynolds number is just 0.3 . It is therefore clear that laminar flow is dominant in microfluidic devices.

Because the Reynolds number is very low for microfluidic devices, it is also common to assume that lateral convection between the fluid streamlines is negligible, so that $\vec{v} \cdot \nabla \vec{v}=0$. Usually the input flow is well controlled and should be stable with time, so we can also assume that $d \vec{v} / d t=0$. Under these conditions Equation (13) simplifies to 


$$
\nabla p=\eta \nabla^{2} \vec{v} .
$$

Equation (14) shows that the pressure force driving the fluid through the channel is exactly balanced by friction, and therefore the fluid is perfectly damped. This extreme case of laminar flow demonstrated at very low Reynolds numbers is referred to as creeping or Stokes flow.

If two separate flows are introduced into a single microchannel, turbulent mixing cannot readily occur, due to the strong viscous forces present. The solutions will instead flow side by side, with mixing occurring only by the slow process of diffusion. This tendency can prove a hindrance in applications which require multiple fluid components to be mixed. Research into techniques to introduce localised turbulent mixing such as channel width and curvature changes [72], networks of posts [73], magnetic particle 'stirrers' [69] and deep wells [74] has helped to combat this problem. On the other hand, the ability to have two flows in direct contact without mixing is attractive for purification applications, including the magnetic separation systems designed for this project.

\subsubsection{Diffusion}

In a microfluidic channel diffusion is the primary mixing mechanism. Diffusion is an entropy-driven process, in which the random Brownian motion of particles will tend towards a uniform particle distribution. This behaviour is clearly seen when a drop of dye is added to water. Even in the absence of convection the dye will gradually spread out, until eventually the whole body of water has changed to a uniform colour.

In two dimensions the average distance a particle is expected to travel from the origin (the diffusion length) is given by [4]

$$
\ell=\sqrt{2 D t},
$$

where $D$ is the diffusion coefficient characteristic of the particle, and $t$ is the time elapsed since the particle was at the origin. The diffusion coefficient for a hard sphere of radius $r$ is given by the Stokes-Einstein relation [75]

$$
D=\frac{R T}{6 \pi N \eta r},
$$


where $R$ is the universal gas constant, $T$ is temperature, $N$ is Avogadro's number and $\eta$ is the dynamic viscosity of the solvent. For example (in water) $D=4 \times 10^{-13} \mathrm{~m}^{2} \mathrm{~s}^{-1}$ for $1 \mu \mathrm{m}$ latex beads, while $D=8 \times 10^{-11} \mathrm{~m}^{2} \mathrm{~s}^{-1}$ for $5 \mathrm{~nm}$ iron oxide nanoparticles; hence the nanoparticles are expected to diffuse much more rapidly. Equation (16) is often used to estimate particle sizes from experimental measurements of the diffusion coefficient, although modifications are sometimes made to improve its accuracy [76].

We may rearrange the terms of Equation (15), in order to consider the diffusion time needed for a solute particle to traverse a given distance

$$
t_{d i f f}=\frac{\ell^{2}}{D} \text {. }
$$

The diffusion time increases with the square of the length scale, meaning diffusion occurs much faster in a microfluidic device than in macroscopic systems. This has been exploited for applications such as the H-filter [4], which separates materials with different diffusion coefficients by allowing them to migrate across a microchannel. As illustrated in Figure 13, smaller ions diffuse more rapidly than larger molecules, and are therefore preferentially selected. For a channel $100 \mu \mathrm{m}$ wide, the process takes only 5 seconds at room temperature, compared to around 14 hours for the sample to diffuse across the width of a standard test tube.

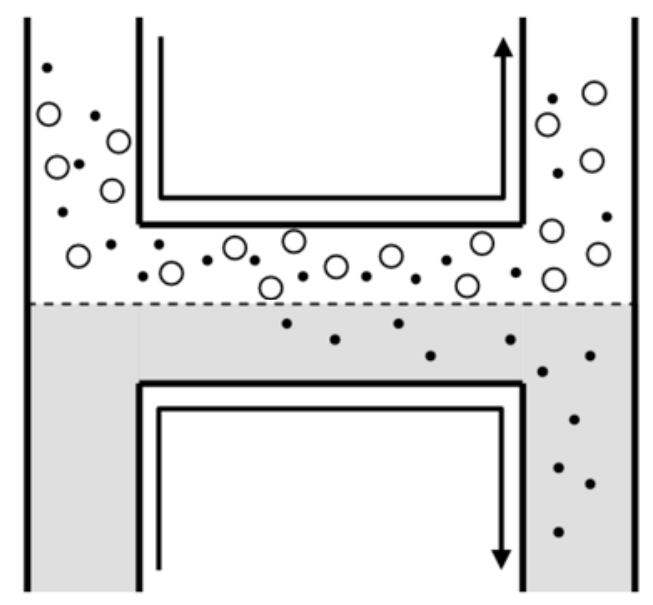

Figure 13: Schematic top-down view of an H-filter, which employs diffusion to separate small ions from larger molecules. The ions (black dots) are able to migrate across the interface between two separate fluid streams much faster than the larger molecules (white circles). Arrows indicate the flow direction of the two fluid streams. Adapted from [4]. 
In applications that do not require mixing to take place, diffusion becomes an undesirable effect that needs to be minimised. In this project, the intention was to transfer particles between adjacent laminar flow streams by applying a magnetic force. Hence thermal diffusion may reduce the selectivity of the method by allowing nonmagnetic particles to diffuse into the collection stream.

We may estimate the severity of this effect, by considering the diffusion of particles from the fluid interface into the buffer stream. It is assumed that the particle concentration at the interface remains at a constant value $c_{0}$. The concentration at a displacement $x$ after a time $t$ is given by [4]

$$
c=c_{0} \operatorname{erfc}\left(-\frac{x^{2}}{\sqrt{4 D t}}\right),
$$

where $\operatorname{erfc}$ refers to the complementary error function, given by

$$
\operatorname{erfc}(s) \equiv \frac{2}{\sqrt{\pi}} \int_{s}^{\infty} e^{-u^{2}} d u
$$

Figure 14 shows the expected concentration profile for spherical $1 \mu \mathrm{m}$ particles at various flow rates, calculated using Equation (18). Flow rates were converted into equivalent transit times by assuming a channel length of $30 \mathrm{~mm}$. From the figure, we see that even at the slowest flow rate diffusion is only on the order of several microns. This compares to a typical channel width greater than $100 \mu \mathrm{m}$, meaning diffusion may be neglected for particles of this size. However, it should be noted that $5 \mathrm{~nm}$ iron oxide nanoparticles may traverse as much as $100 \mu \mathrm{m}$ under the same conditions, meaning faster flow rates would be needed to reduce diffusion to acceptable levels. 


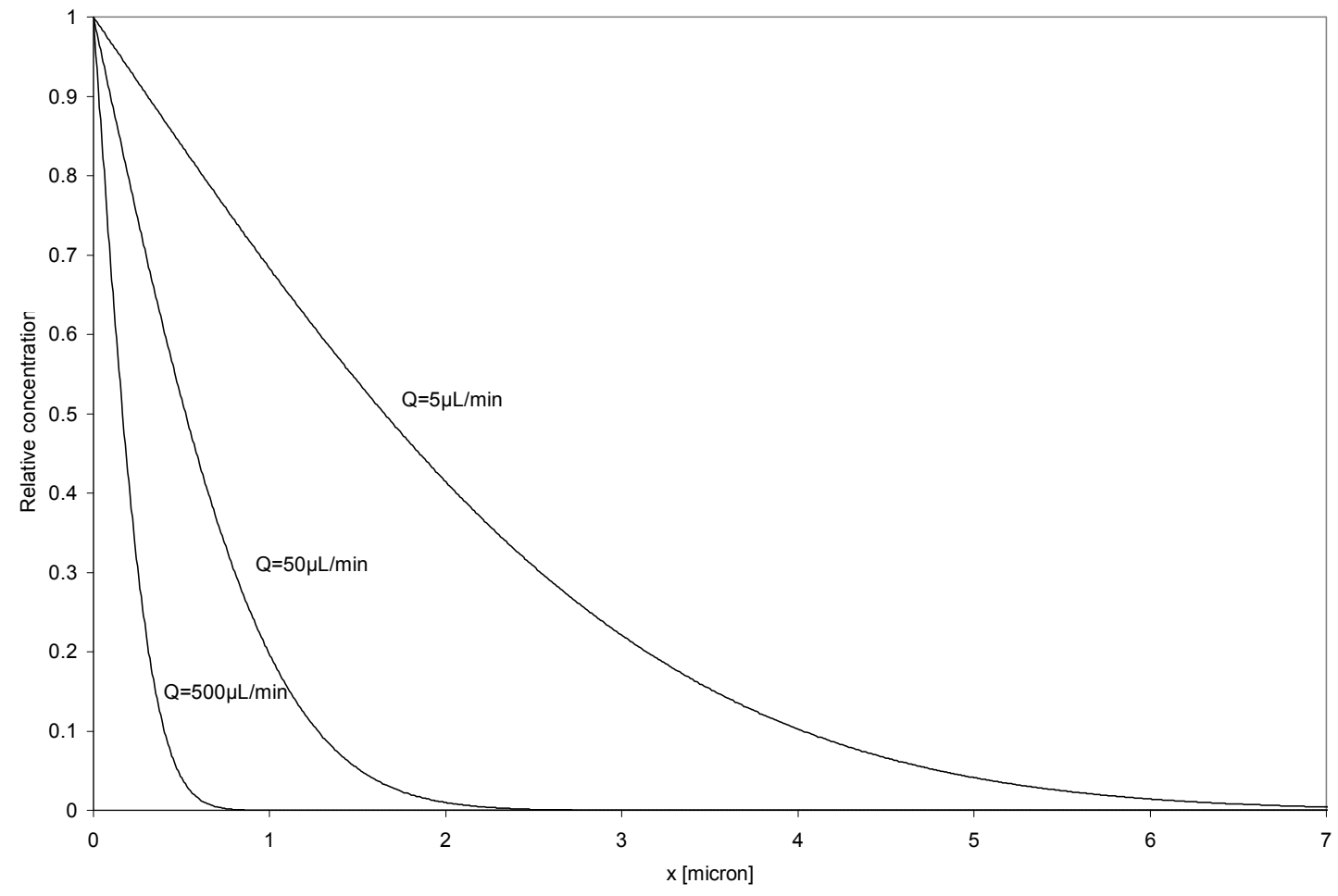

Figure 14: Concentration distribution for $1 \mu \mathrm{m}$ particles diffusing from a point source in 1D. Distributions are shown for multiple flow rates, based on a channel length of $30 \mathrm{~mm}$.

\subsubsection{Device fabrication}

Microfluidic devices have been made from multiple material types, each requiring different fabrication methods. In this section, some of the most popular approaches to device fabrication are briefly introduced.

Microfluidics was initially viewed as an extension of MEMS systems, and hence early devices were fabricated in silicon. This allowed the wealth of fabrication techniques developed for the microelectronics industry to be adapted for making microfluidic structures. Micromachining of silicon is usually achieved through photolithography, followed by wet etching in hydrofluoric or nitric acid [77]. Dry etching processes such as reactive-ion etching, and the more advanced Bosch process, enable high-aspect ratio structures to be developed [78]. Silicon micromachining requires cleanroom conditions to operate, making it unsuitable for small-scale research. Silicon is also too expensive to use in disposable devices, and its opacity makes it incompatible with optical detection methods. Silicon remains a popular material for the creation of templates [79-82], which can then be used to create disposable devices in plastics. 
Glass is a cheaper alternative to silicon, and can be machined using many of the same techniques. Unlike silicon it is optically transparent, allowing the channels to be observed. Glass is compatible with a wide range of solvents, as well as live biological samples. Devices made from glass are durable, making them ideal for reusable devices [83-85]. However, the material and machining costs are still significant, making them impractical for most single-use applications.

Demand for inexpensive disposable microfluidic devices has led to the emergence of plastics as the preferred structural material. As well as reducing the material cost, plastics allow a number of inexpensive and rapid fabrication techniques to be employed.

Laser ablation involves the use of a high-powered ultraviolet or infrared laser to selectively remove material from a thermoplastic. In some cases a lithographic mask is used to determine the regions to be exposed, allowing the entire substrate to be treated simultaneously [86]. It is however more common to use a direct-write process, in which a programmable translation stage is used to draw the desired pattern into the substrate [87]. This reduces the required laser power, at the cost of increased fabrication time. Ablation is a useful technique for prototyping, but throughput issues limit its use for bulk fabrication.

Embossing uses a stamp made from silicon or metal to pattern features into a thermoplastic. A hydraulic press is used to force the stamp against the plastic, which will then mould to the features of the stamp [82]. Room-temperature embossing at higher pressures has also been demonstrated [79]. Embossing is suitable for the rapid reproduction of identical structures, as the same stamp can be repeatedly used. Creating stamps in silicon or metal is time-consuming, which makes the process unattractive for prototyping.

Injection moulding is a popular process for mass-production of microfluidic structures, and is commonly used to produce commonplace plastic products $[88,89]$. In this process a thermoplastic is heated above its glass transition temperature, and the liquid injected at high pressure into a mould. The plastic is then cooled and solidifies, before being 
removed from the mould. Injection moulding allows for excellent feature reproduction, and components can be produced in just a few minutes. However, the need for complex moulding equipment, as well as moulds similar to those used in embossing, makes the process impractical for small-scale fabrication.

The most popular fabrication technique for small-scale research applications is known as replica moulding. This involves pouring a plastic or elastomeric liquid onto the surface of a patterned master. The liquid conforms to the features on the master, and is then cured under moderate heat to form a solid. High temperatures are not required, and the masters may therefore be made from inexpensive materials such as PMMA [90] rather than silicon or metal. For devices requiring strong chemical resistance, thermoset polyesters [91] or polyimides [92] are often used as the moulding material. In applications using only aqueous solution, polydimethylsiloxane (PDMS) is the material of choice [93]. In particular, the optical transparency, low cost, and elastomeric nature of PDMS led to its use in this project. The production of microfluidic devices in PDMS is referred to as soft lithography, and this process is described in further detail in Section 3.1 .

\subsubsection{Flow control}

Most microfluidic systems require a method to introduce a flow of solution into the channels. Often it is required to ensure that the flow is continuous, steady and welldefined. The two most common flow types are pressure-driven flow and electrokinetic flow, which are used with about $90 \%$ of current device applications [94]. Both techniques have their own benefits and drawbacks, which are discussed below.

Pressure-driven (Poiseuille) flow relies on a pumping mechanism in order to create a pressure difference along the length of a microchannel. This pressure difference then leads to a flow from the high-pressure to the low-pressure end of the channel. The pressure difference is commonly applied externally, usually by means of a mechanical syringe pump. A syringe pump drives or withdraws the flange of a syringe at a defined rate which, provided the syringe geometry is well-known, translates to a defined volumetric flow rate. Tubing and luer adaptors (see Section 3.1.4.) are commonly used 
to connect syringes to the fluid channels, although in some cases tubing is directly connected to the microfluidic chip. The driving force of the pump is designed to be steady to ensure a stable flow, although friction or misalignment of the syringe may cause disruptions. This was the flow-control method used in this project, motivated by its availability, simplicity and low cost. A major disadvantage is that considerable pressure is exerted on the device walls, which may rupture the channels.

It is interesting to note that the velocity of the fluid is not uniform across the width of the channel. It instead follows a parabolic distribution, with the fluid flowing fastest at the centre [95], as shown in Figure 15. This arises due to the no-slip condition, which holds that the fluid velocity at the channel walls is zero [96]. It has been suggested that a small amount of slip occurs in practice, depending on the hydrophobicity of the walls [97], but the effect on the flow profile is insignificant.

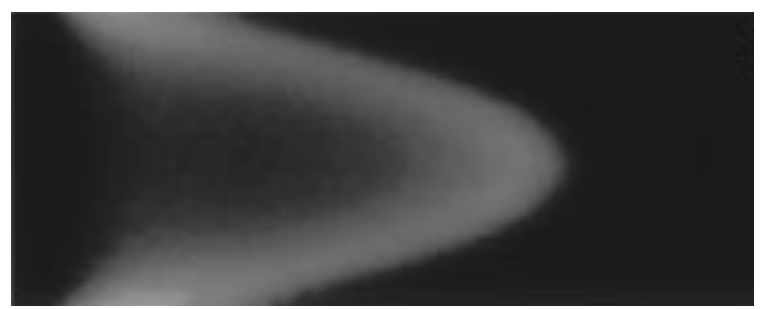

Figure 15: Image of pressure-driven flow in a $100 \mu \mathrm{m}$ diameter silica capillary, $231 \mathrm{~ms}$ after releasing the dye. A parabolic flow profile is obtained [95]. Flow direction is from left to right of image.

Research into the miniaturisation of pressure-driven flow systems is ongoing, in order to provide pumping to integrated Lab-on-a-chip devices. Nagel et al [98] impregnated a PDMS microfluidic device with magnetic microparticles, enabling a flow to be actuated using an external magnet. Miniaturised pressure-driven flow has also been demonstrated by Unger et al [99], by using dry channels as pneumatic valves in a PDMS device. More advanced techniques, such as piezoelectric-actuated pumps [100] driven by an external voltage, have also been demonstrated. Integrating moving parts into microfluidic devices has proven difficult, due to problems with mechanical fatigue and defects [101]. 
Electrokinetic flow, also known as electro-osmotic flow, relies on the polarity of the fluid itself in order to create a flow. Interactions between the channel walls and solvent will often produce local surface charges [101], which then attract opposing charges from the solvent, forming what is known as an electric double layer (EDL). This layer is of the order of nanometres in width, with the bulk solution remaining neutral. If an electric field gradient is applied along the length of the channel, the ions in the EDL will migrate to one electrode. Viscous forces between the molecules will then drag the bulk solution along with it. Provided the channels are open at either end to prevent backflows, this effect gives a uniform flow profile across the width of the channel [95], as shown in Figure 16. The no-slip condition is still obeyed, but the transition occurs over the much smaller EDL width as opposed to the entire width of the channel. Because electroosmosis obviates the need for moving parts to create a flow, it readily lends itself to miniaturisation. All that is required is a voltage source that can sustain a sufficient electric field gradient along the channel.

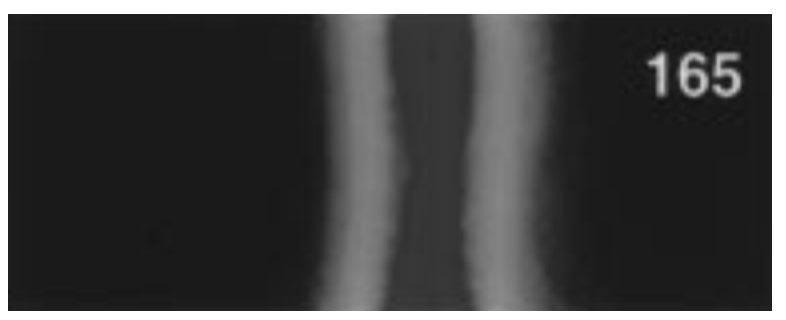

Figure 16: Image of electro-kinetic flow in a $100 \mu \mathrm{m}$ diameter silica capillary, $165 \mathrm{~ms}$ after releasing the dye. A uniform flow profile is obtained [95]. Flow direction is from left to right of image

Although pressure-driven flow and electrokinetic flow are the most commonly used methods, several alternatives exist. Centrifugally-induced pressure can be used to drive small quantities of fluid. The so-called Lab-on-a-CD [102] system uses channels patterned on a substrate shaped like a compact disc, allowing a standard CD drive to apply the force needed. Surface-tension driven flow exploits the large capillary forces present in microchannels to induce flow. The simplest case is passive pumping [103], in which the fluid spontaneously wets the channel. Continuous capillary pumping can also be realised, by using electric fields to modulate the surface energy of the channels [104]. Gravity has been successfully used to drive flows in microfluidic channels [105], although the flow rate cannot be controlled, and in most cases surface tension will 
prevent flow. Finally, acoustic waves have shown promise for controlling fluid flow, but the technique is so far underdeveloped [106].

\subsection{Forces on a magnetic particle}

Magnetic particles suspended in a fluid are subject to several competing forces during a separation experiment. The most important of these are gravity, fluid drag and the applied magnetic force. These forces in particular will influence the rate at which magnetic separation can be achieved, as well as the length of time for which particles will remain in suspension without settling. In this section, basic mathematical descriptions for estimating the strength of these forces will be discussed, and the balance of these forces used to predict the velocity of the magnetic particles. In addition, the significance of Brownian motion and particle agglomeration will be considered.

\subsubsection{Gravity}

In all instances a particle will experience a net gravitational (or buoyancy) force of magnitude

$$
\vec{F}_{g}=V_{p} \Delta \rho \vec{g}
$$

where $V_{p}$ is the volume of the particle, $g$ is the gravitational force and $\Delta \rho$ is the difference in density between the particle and surrounding medium. Gravity does not contribute to the separation process directly, as the applied magnetic field was used to direct particles in the plane normal to the gravitational field. Gravity nonetheless needs to be considered, as it will place an upper limit on the time the particles may spend in a microfluidic channel without settling. For $1 \mu \mathrm{m}$ magnetic beads $\left(\rho=1.8 \mathrm{~g} / \mathrm{cm}^{3}\right)$ the net force on each bead is $4.2 \mathrm{fN}$.

\subsubsection{Fluid drag}

A particle moving through a fluid will experience a drag force, due to friction at the particle-fluid interface. As discussed in Section 2.3.2, the length scales characteristic of microfluidic devices correspond to extremely small Reynolds numbers $(\operatorname{Re}<1)$, meaning it is appropriate to use Stokes' law to simply describe the drag on a particle as [4]

$$
\vec{F}_{d}=6 \pi r_{p} \eta \Delta \vec{u},
$$


where $r_{p}$ is the particle radius, $\eta$ is the dynamic viscosity of the fluid, and $\Delta u$ is the velocity of the particle relative to the fluid. Fluid drag will hinder any separation process, by opposing the motion of particles arising from an applied magnetic field; hence stronger field gradients (or longer separation times) are needed in more viscous media. On the other hand, fluid drag reduces the rate at which particles settle under gravity, allowing for longer experimental times.

\subsubsection{Magnetic force}

The magnetic force experienced by the particle is given by [107]

$$
\vec{F}_{\text {mag }}=\mu_{0} \int_{V}(\vec{M} \cdot \nabla) \vec{H}_{0} d v
$$

where $M$ is the particle magnetisation and $H_{0}$ is the externally applied field. The integral is taken over the volume of the particle. Because the particles used in this project are of size $5 \mu \mathrm{m}$ or less, we can assume that the applied field is constant over the volume of the particle, and hence Equation (22) simplifies to

$$
F_{\text {mag }} \approx \mu_{0} V(M \cdot \nabla) H_{0}=V M(H) \vec{G} .
$$

The magnetisation $M(H)$ is a non-linear scalar function, corresponding to the magnetisation curve of the material. We have also used the effective field gradient, defined by Smistrup [108] as

$$
\vec{G} \equiv \mu_{0}\left(\frac{\vec{H}}{H} \cdot \nabla\right) \vec{H} .
$$

Equation (23) illustrates the direct dependence of the magnetic force on the gradient of the applied field. A uniform field would induce a torque, but no translational motion, regardless of its strength. The definition stated in Equation (24) is a generalisation to three dimensions, and includes cases where the applied field possesses finite curl. In this project however, field gradient data from simulations could only be exported for positions along a tangent line. Calculations were therefore made in one dimension, and assumed a field with zero curl. Under these conditions, the bracketed term in Equation (24) is always equal to 1 , and the gradient operator may be replaced by a simple derivative, yielding

$$
G=\mu_{0} \frac{d H}{d x}
$$


In the case where the applied field $H$ is in units of tesla, the field gradient $G$ is expressed in units of $\mathrm{T} / \mathrm{m}$. Because $G$ is independent of particle type, it is commonly used to provide a rough comparison between different separation systems.

The use of Equation (23) to predict particle motion requires knowledge of the magnetisation behaviour of the material. This can be readily ascertained through magnetometry, but this adds an often unnecessary layer of complexity to the process. Considering the low and high-field limits of Equation (23) often allows us to estimate the magnetic force using less information about the material.

For superparamagnetic materials in low applied fields, the magnetisation curve is essentially linear. Hence $M=\chi H$, where the magnetic susceptibility $\chi$ is constant. Using this, along with the result of Equation (25), Equation (23) may then be rewritten as

$$
F_{\text {mag }}=\frac{1}{2} \mu_{0} V \chi \frac{d}{d x} H^{2} .
$$

The low-field limit expressed in Equation (26) is not appropriate for ferromagnetic materials or intermediate fields, where the magnetisation behaviour is no longer linear. However, it is useful when superparamagnetic materials are manipulated using small electromagnets [109-111], from which the stray field is generally small.

A ferromagnetic or superparamagnetic material will approach magnetic saturation at high fields. Therefore, provided the applied field is large enough, we can assume $M(H)=M_{s}$, where $M_{s}$ is the saturation magnetisation of the material, and Equation (23) simplifies to

$$
F_{\text {mag }}=\mu_{0} V M_{s} \frac{d}{d x} H
$$

The systems used in this project all rely on strong permanent magnets to provide a field, with surface fields in excess of $0.4 \mathrm{~T}$. Hence in all cases it was assumed that Equation (27) was the most appropriate for force calculations. In a readily-attained field gradient of $60 \mathrm{~T} / \mathrm{m}$, the force on a MyOne magnetic bead $\left(\mathrm{V}=6.05 \times 10^{-19} \mathrm{~m}^{3}, \mathrm{M}_{\mathrm{s}}=4.76 \times 10^{4}\right.$ $\mathrm{A} / \mathrm{m})$ is approximately $1.7 \mathrm{pN}$. 


\subsubsection{Brownian motion}

As discussed in Section 2.3.3, particles in solution will undergo random thermal motion, due to collisions with surrounding particles. In the absence of other forces, a given particle will not tend towards a particular direction, but will nonetheless move in a random walk away from the origin (see Equation (15)). If this motion is comparable to or larger than the magnetically induced motion, particle capture may be compromised.

A detailed treatment of the effects of Brownian motion on magnetic separation is provided by Friedman and Yellen [112]. The authors considered the work done by gravitational and magnetic forces in moving a particle over a distance of one diameter. They then compared this to the thermal energy, which is given by

$$
E_{\text {thermal }}=k T \text {, }
$$

where $k$ is Boltzmann's constant and $T$ is temperature. Thermal motions are randomly oriented, rather than acting coherently in a single direction. Gravitational and magnetic forces, on the other hand, are applied consistently in one direction. We can therefore assume these forces dominate the motion of the particle, provided the work done is comparable to or larger than the thermal energy. At room temperature, the thermal energy is equal to $4.1 \times 10^{-21} \mathrm{~J}$. As stated in Section 2.4.1, the gravitational force on a 1 $\mu \mathrm{m}$ magnetic bead is $4.2 \mathrm{fN}$, meaning $4.4 \times 10^{-21} \mathrm{~J}$ of work is done by the gravitational field. These energies are comparable, meaning Brownian motion may slow down the sedimentation of the beads. A readily-achieved magnetic force of $1.7 \mathrm{pN}$ (see Section

2.4.3) corresponds to $1.8 \times 10^{-18} \mathrm{~J}$ of work. This is several orders of magnitude greater than the thermal energy, indicating Brownian motion will have a negligible impact on magnetic separation for this type of particle.

\subsubsection{Particle agglomeration}

As discussed in Section 2.4.1, the gravitational force on a particle increases with its volume. In a solution containing many particles, the possibility of particles collecting into large agglomerates is a concern, as this may lead to very rapid sedimentation. Particles continually undergo collisions, meaning agglomeration is expected if the contact energy between particles dominates the thermal energy. A number of factors, including electrostatic or Van der Walls attraction, may contribute to the contact energy. 
However, it is assumed here that for magnetic particles this energy is dominated by the magnetic dipole attraction [49], as given by

$$
E_{d d}=\frac{1}{12} \mu_{0} M^{2} V
$$

By considering the ratio of the thermal energy (see Equation (28)) to the dipole energy, we can then estimate the maximum particle diameter that will not agglomerate as [49]

$$
d<\left(72 k T / \pi \mu_{0} M^{2}\right)^{1 / 3} .
$$

It should be noted that the dipole energy is zero if the particles have zero magnetisation, meaning true superparamagnetic particles should not agglomerate in the absence of an applied field. Ferromagnetic particles will however have a finite remanence, which may promote agglomeration.

\subsubsection{Particle velocity}

The results of Sections 2.4.1-2.4.3 may be used to estimate the velocity of a magnetic particle in a separation system. Specifically, the balance between the magnetic and fluid drag forces on a particle is considered. It is common to neglect particle inertia, on the grounds that terminal velocity is reached almost instantaneously at this scale [112]. The characteristic length scales of the field gradients used in this project are of the order of millimetres, essentially constant on the micron scale, meaning that the particle will always travel at its terminal velocity.

At terminal velocity, the fluid drag and magnetic forces are balanced, hence setting Equations (21) and (27) equal and solving for velocity leads to

$$
u_{\text {mag }}=\frac{F_{m a g}}{6 \pi \eta r} \approx \frac{V M_{s} G}{6 \pi \eta r} .
$$

The equation has been simplified by assuming the high-field form of $F_{\text {mag }}$, although any form may be used. It is interesting to consider the ratio in velocities between two particle types in the same separation system, which in the high-field limit is given by

$$
\frac{u_{1}}{u_{2}}=\left(\frac{M_{s, 1}}{M_{s, 2}}\right)\left(\frac{r_{1}}{r_{2}}\right)^{2} \text {, }
$$


where $M_{s, 1}$ and $M_{s, 2}$ are the saturation magnetisations of particles 1 and 2 respectively, and $r_{1}$ and $r_{2}$ are the particle radii. If all particles have the same magnetisation, then a particle with twice the radius will move at four times the speed in the applied field. If all particles are the same size, then a particle with twice the saturation magnetisation will move twice as fast in the field. The significance of this result is that magnetic separation may be used to isolate particles of a particular size and/or magnetisation. The ratio described in Equation (32) is commonly called the magnetophoretic mobility, as it allows us to compare the ease with which different particle types may be separated in a given field gradient.

\subsection{Magnetic Separation}

Magnetic separation refers to the isolation of a material from one or many others, achieved by exploiting differences in their magnetisation. In the context of this thesis, the term specifically refers to the extraction of micron and sub-micron sized ferromagnetic and superparamagnetic particles from a diamagnetic solution. The motivation for such a process can be either the enrichment of a sample of magnetic particles, or the isolation of a target to which the magnetic particles selectively bind.

This section will provide an overview of the current techniques and systems available for the separation of magnetic microparticles and nanoparticles. The first subsection will introduce so-called 'benchtop' systems, which offer relative design simplicity and high throughput, but lack sensitivity. The second subsection will discuss various microfluidic systems, which are more complicated, but offer significant sensitivity and portability improvements. All the systems discussed here rely on creating a sufficiently large magnetic field gradient to alter the flow of magnetic particles in solution.

\subsubsection{Benchtop systems}

The simplest example of a benchtop magnetic separation system is illustrated in Figure 17. A magnet is used to extract magnetic carriers from a test tube of solution, by causing agglomeration of the particles against the sidewall, allowing the supernatant to be discarded. In some biomedical applications, magnetic particles are functionalised to bind to specific cell types, allowing the concentration of nonmagnetic cells. This process, known as magnetic solid-phase extraction (MSPE), has been used to refine red blood 
cells, cancer cells and bacteria [113]. This approach features relatively weak field gradients (1-6 T/m [114]), meaning relatively high particle concentrations and/or capture times are needed for effective separation.

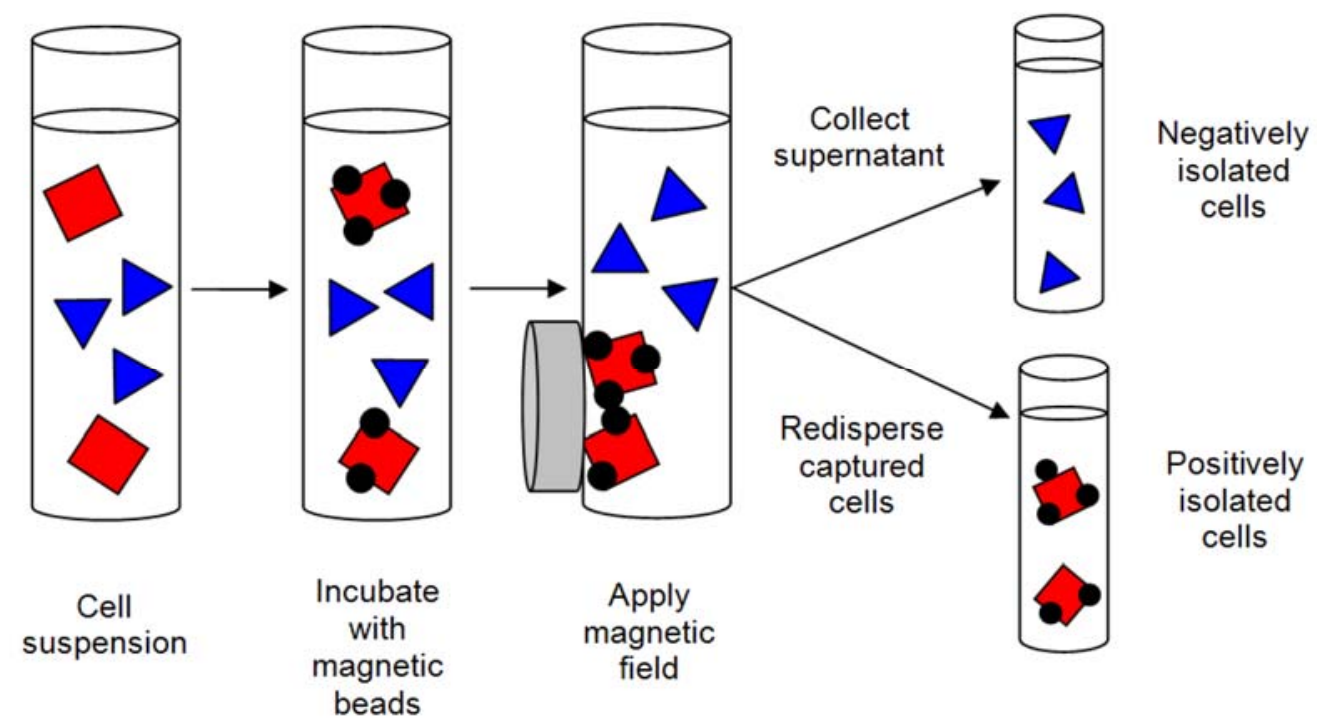

Figure 17: Schematic representation of the magnetic separation of cells. Magnetic microbeads are added to a suspension of cells. The surfaces of the beads are functionalised to chemically bind to a single cell type. A magnet draws the magnetic beads out of solution, along with the bound cells. The supernatant is then removed and the captured cells redispersed.

High-gradient magnetic separation systems (HGMS) were first introduced in the mid1970s [2] for the removal of large particles from industrial wastes. Their ability to capture even sub-micron particles meant they also showed potential for use in laboratory processes [115]. HGMS systems generally utilise a mesh of steel wool, wire or ball bearings, creating very strong local field gradients in the presence of an applied field. Using the same field strengths as MSPE, field gradients of 10-100 T/m can be achieved [114]. The operating principle of HGMS is illustrated in Figure 18. The large field gradients make it possible to capture particles as the fluid flows through the magnetised mesh. The applied field is then removed, and the particles recovered. One of the best examples of a modern-day HGMS system is the commercially available range of MACS separators (Miltenyi Biotech, Germany [54]) used in biotech research. 


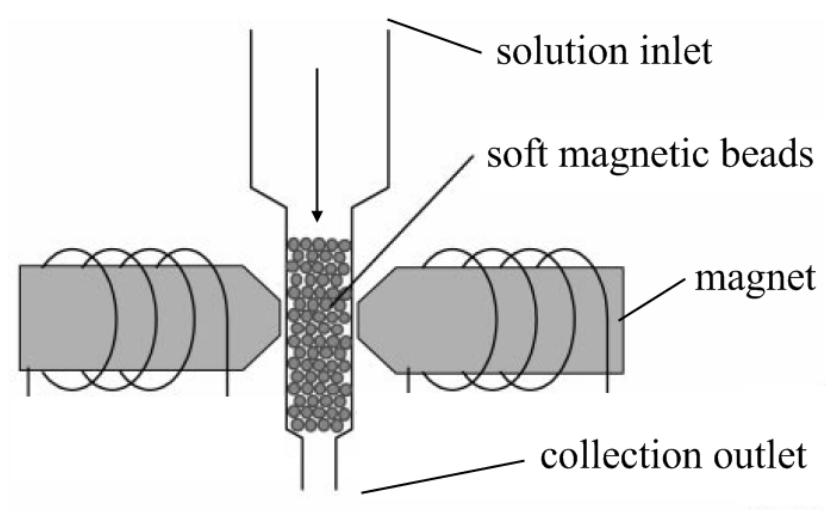

Figure 18: Operating principle of a typical high-gradient magnetic separation (HGMS) system. Flux from the external magnet is concentrated by the soft magnetic elements inside the column to provide large field gradients. Image adapted from [116].

HGMS do carry limitations that may limit their use. Particle build-up on the mesh leads to increased adsorption of incoming particles [117]. Therefore, as the run proceeds efficiency will increase, as magnetic particles are more likely to be captured. At the same time, selectivity will decrease, as non-magnetic particles are also more likely to be caught. Particles may also become permanently trapped if the mesh material has a significant magnetic remanence.

Modifications to HGMS to allow fractionation of magnetic fluids have been developed in recent years, allowing samples with more uniform magnetic properties to be prepared. This is in contrast to standard HGMS, which is designed to capture all magnetic material. A system designed by Rheinlander et al. [116] used a powerful electromagnet (B 1T) in place of a permanent magnet, allowing the field to be decreased in steps. Particles of high magnetophoretic mobility remained bound to the mesh down to lower applied fields. Such systems have been used to optimise nanoparticle solutions for hypothermia research $[118,119]$.

An alternative approach to achieving very large magnetic field gradients for separation was demonstrated by Todd et al. [120]. The distance the particles were required to migrate was reduced by using a multistage separator design, in which magnets of increasing strength were brought very close to the fluid. Field gradients were reduced compared to HGMS $(<15 \mathrm{~T} / \mathrm{m})$, requiring long dwell times of 15 minutes per step, but 
the process was entirely automated. While effective, this complicated design is likely to be expensive, and is not portable.

\subsubsection{Microfluidic systems}

As discussed in Section 2.3.1, microfluidic systems are appealing in two regards. Firstly, the small length scales lead to laminar flow, meaning mixing occurs solely by diffusion. Secondly, they allow for the possibility of performing a series of laboratory processes on a single chip, requiring a minimal solution volume. It is also substantially easier to produce a strong magnetic field gradient across a microfluidic channel than a test tube, due to the smaller length scales involved. This section will describe the main types of microfluidic magnetic separation systems, and present some notable examples.

It is helpful to characterise the various device design approaches on the basis of a few key aspects. The first is the method by which the particles are collected, which leads to the following three categories:

- Particle capture - Particles introduced are exposed to overwhelming field gradients, causing settling of the particles against the channel floor or sidewalls, and thus removing them from the solution. The field gradient is subsequently removed, allowing the particles to be flushed out of the device. Particle capture devices generally feature simpler designs, as only a single channel is required. Fine-tuning of the applied field gradient is also not needed, as the maximum achievable field gradient is generally used. A drawback of this approach is that particles may eventually build up and clog the channel. Devices may also be restricted to use with paramagnetic or superparamagnetic material, as ferromagnetic materials may remain agglomerated even when the field is removed.

- Continuous flow - Particles introduced are exposed to a smaller field gradient, sufficient to divert the particles into an adjacent fluid stream without removing them from the solution. Material is thus collected from the device at the same rate it is introduced. Continuous flow separation ideally does not lead to particle build-up, and is therefore compatible with large volumes of material. The possibility of sorting multiple particle types is also afforded. However these 
advantages come at the cost of more complicated channel geometries, and the need to fine-tune the field gradient to avoid inadvertently trapping particles.

- Particle transport - Particles are no longer moved through the microfluidic channels by the fluid flow, but instead transported from one region to the next by a series of magnetic field gradients. Fine control is required to accurately manipulate the particles, meaning electromagnets are invariably used to provide the field gradient. Designs of this type are of particular interest for use in complex Lab-on-a-Chip systems, where avoiding the need for an integrated pumping mechanism is desired.

The second key design aspect is the source of the field gradient. The three most common sources are

- Permanent magnets - The simplest example of this approach involves placing a handheld magnet in the vicinity of the device. Large fields $(>0.3 \mathrm{~T})$ are easily obtained, although in order to achieve large gradients it is preferable to integrate miniature magnets into the device itself. Field gradients in excess of $100 \mathrm{~T} / \mathrm{m}$ can be obtained, with no heat produced. However, fine positioning of the magnets can be challenging, and the field may not be switched off without physically moving the magnet away from the channel.

- Electromagnets - Many processes used to fabricate microfluidic devices are based on processes used in microelectronics, meaning that precise integration of current lines can be readily achieved. These features can be positioned to the channel to provide a field gradient. The field gradient may be easily controlled by varying the applied current, but in most cases Joule heating will limit the maximum gradients that can be achieved to a few tens of $\mathrm{T} / \mathrm{m}$. In addition, the fields produced are generally too small to magnetically saturate the particles, further reducing the strength of the magnetic force.

- Passive structures - These consist of soft magnetic features integrated into the device. These features provide no attractive force unaided, but will concentrate an external field to produce strong localised field gradients. In this sense, the operating principle is similar to that of HGMS, which was introduced in Section 2.5.1. These systems present an appealing middle ground between the first two 
approaches, as there is no need to physically touch the device to turn the field gradient on and off, and no heating problems are encountered. Integration of these elements may however require techniques such as metal evaporation, which can be costly to implement.

A non-exhaustive literature summary of microfluidic magnetic separation systems is presented in Table 1. References are categorised according to the design approaches discussed above. Systems of particular interest are also briefly discussed.

\begin{tabular}{|c|c|c|}
\hline \multirow{2}{*}{ Type } & Gradient source & References \\
\hline \multirow{4}{*}{ Capture } & Permanent magnet & {$[121,122]$} \\
\cline { 2 - 3 } & Electromagnet & {$[123-128]$} \\
\cline { 2 - 3 } & Passive & {$[129-132]$} \\
\hline \multirow{3}{*}{ Continuous } & Permanent magnet & {$[133-138]$} \\
\cline { 2 - 3 } & Electromagnet & {$[109,110,132]$} \\
\cline { 2 - 3 } & Passive & {$[113,139-142]$} \\
\hline Transport & Electromagnet & {$[143-150]$} \\
\hline
\end{tabular}

Table 1: Some notable examples of microfluidic magnetic separation systems, organised by the separation method and the source of the magnetic field gradient.

Xia et al. [113] described a PDMS-on-glass system for continuous separation of magnetic beads, which is presented in Figure 19. The solution containing the particles and a separate buffer solution were brought into direct contact without mixing. Application of a magnetic field gradient then drew the magnetic beads into the buffer solution. A microfabricated layer of $\mathrm{NiFe}$ concentrated the magnetic field from a permanent magnet, producing field gradients of up to $250 \mathrm{~T} / \mathrm{m}$ inside the fluid channel. The device is biocompatible, and was used to recover live E-Coli bacteria bound to magnetic beads.

$\mathrm{Bu}$ et al. [129] presented a system for trapping magnetic beads which is suitable for high-throughput applications. As shown in Figure 19, a single wide fluid channel was 
situated above an array of Permalloy strips. Small permanent magnets positioned under the device drew the beads towards the channel floor, and also magnetised the Permalloy strips. The magnetised strips then provided very strong short-range field gradients, which trapped the particles and prevented them from being swept away by the fluid flow. Because the particles are drawn towards the bottom of the channel, the channel can be made very wide without affecting the separation efficiency. Flow rates as large as $1 \mathrm{~mL} / \mathrm{min}$ may therefore be used with this device.
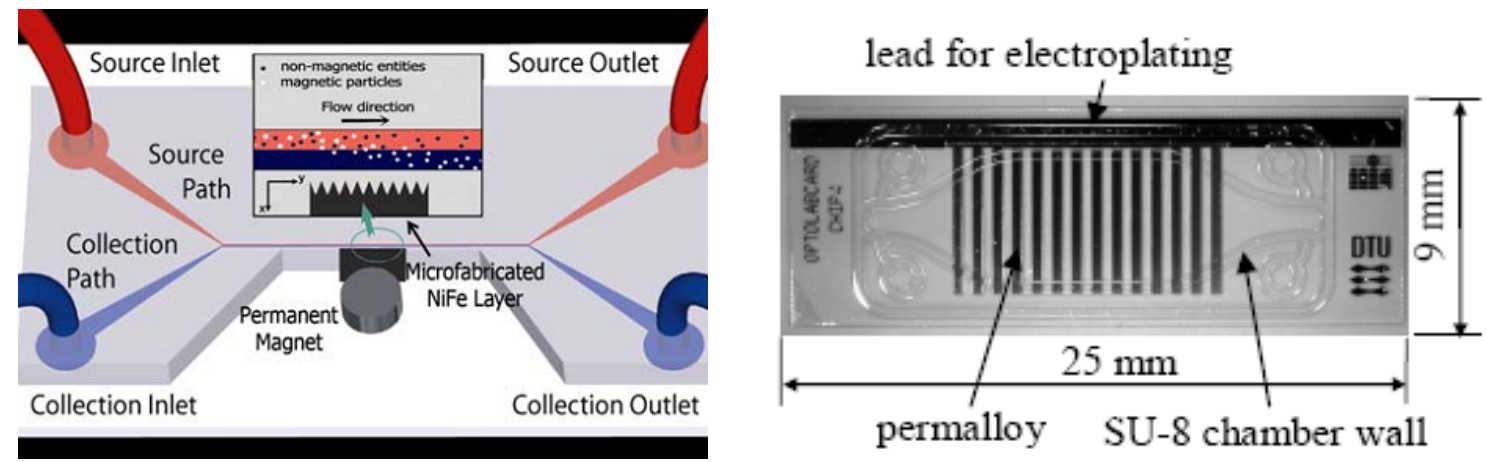

Figure 19: (Left) Operating principle of a continuous magnetic separation device designed by Xia et al. [113]. (Right) Top-down view of a bead capture system developed by Bu et al. [129].

Siegel et al. [110] demonstrated a convenient method for producing on-chip electromagnets in PDMS without expensive microfabrication techniques. The method involves patterning additional channels into the PDMS, and then injecting these with liquid solder. Once solidified, the solder forms a wire, which will produce a magnetic field when a current is passed through it. This allows electromagnets to be produced in very close proximity to the fluid channels, which partly compensates for the small fields produced. The group successfully diverted a stream of superparamagnetic beads into a single outlet of a channel junction using this technique.

A continuous separation system capable of sorting magnetic beads on the basis of size and/or magnetic moment was developed by Pamme et al. [134]. As shown in Figure 20 the device contained multiple inlets and outlets. The solution containing the sample was introduced through a single inlet, with a buffer solution pumped into the remaining inlets. Several permanent magnets were placed near the chip, and supplied a field gradient perpendicular to the direction of fluid flow. Magnetic particles were drawn towards the magnets, and exited the device at one of the collection outlets. Larger or 
strongly magnetic particles exited closer to the magnet than smaller or weakly-magnetic particles.

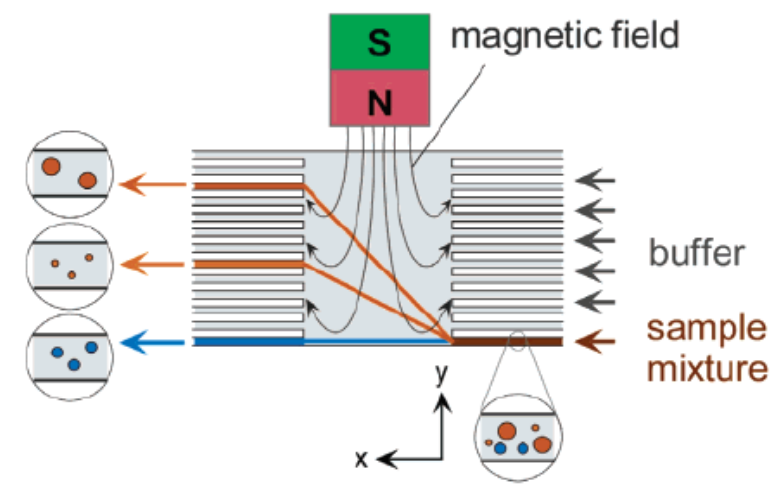

Figure 20: Schematic of the continuous magnetic separation system developed by Pamme et al. [134]

Ramadan et al. [125] present a thorough analysis of multiple coil designs for the capture of magnetic beads in solution. A complete microfluidic system suitable for the capture of biological cells based on these coils was later developed [126]. This system generated field gradients as large as $3 \times 10^{4} \mathrm{~T} / \mathrm{m}$, which enabled around $80 \%$ of the magnetic beads to be captured [126]. At the same time another system was developed, which used a series of coils to enable transport of magnetic beads along the length of a microfluidic channel [145]. Similar systems developed by Lee et al. [147] used a wire matrix to manipulate individual magnetic beads. By controlling the wire currents, single or multiple peaks in the magnetic field could be generated and independently moved in two dimensions. Magnetic beads are attracted to the field maxima, allowing them to be controlled with micrometre precision. As shown in Figure 21, this system was capable of manipulating individual yeast cells bound to magnetic beads $[149,150]$.
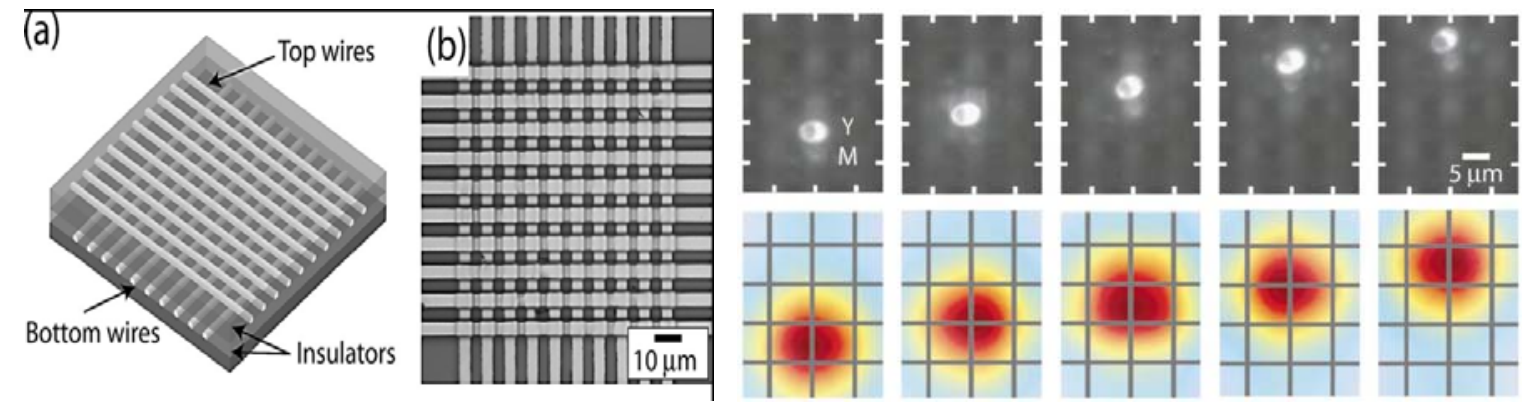

Figure 21: (Left) Matrix design used by Lee et al. [149] for manipulating magnetic beads. (Right) Transport of a single yeast cell (Y) attached to a magnetic bead (M). Simulations of the magnetic field distribution are shown under each frame, confirming that the bead follows the peak in the magnetic field. 


\section{Experimental}

This chapter provides details of the experimental techniques used during this project. The development of microfluidic devices by soft lithography is first discussed in Section 3.1. Magnetic nanoparticles were used in some of the magnetic separation experiments, and their synthesis is therefore described in Section 3.2. This is followed by a summary of the methods used to control the flow of solution in the channels of microfluidic devices, and the techniques used to characterise magnetic nanoparticles, microbeads and liquids. Finally, the procedures used to examine the effect of microfluidic channels on fluid flow, as well as their use in magnetic separation, are presented in Section 3.6.

\subsection{Microfluidic device design and fabrication}

A core aspect of the experimental work conducted during this project was the development of a microfluidic system suitable for magnetic separation. Microfluidic devices were produced using a low-cost process known as soft lithography. This involves the creation of a permanent 'master' using a photoresist, which is then used to mould a 'replica' in a suitable polymer. The approach used here is similar to that described in detail by Duffy et al. [5] and McDonald et al. [151]. Devices were produced in standard laboratory conditions, without the use of cleanroom facilities or specialised equipment.

The experimental procedure was based on a protocol developed by Chooi [152], and the reader is referred to the resulting Honours project report for further details of the method. The content of this section will be limited to a general overview, with emphasis placed on changes made to improve the robustness of the resulting microfluidic devices. Many of these changes were required in order to achieve consistent results when using the devices for the separation of magnetic particles. A process flow diagram, which summarises the key steps of the procedure, is presented in Figure 22. 


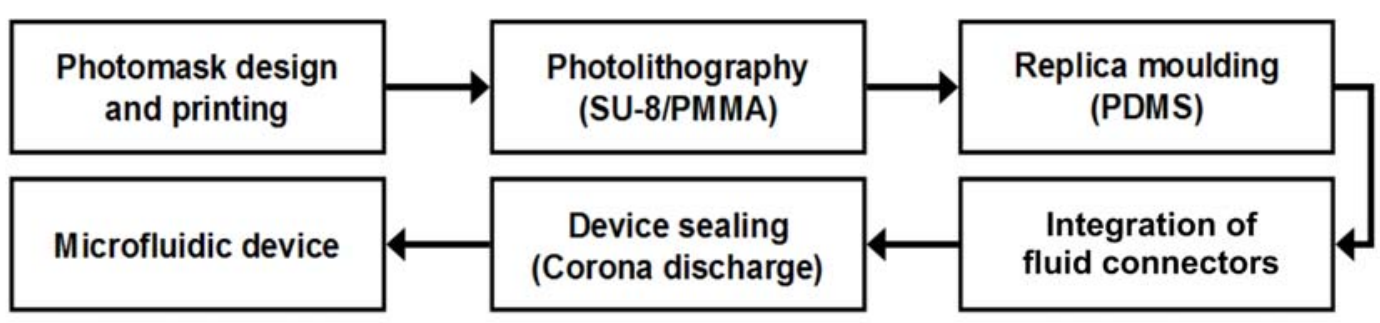

Figure 22: Process flow diagram for microfluidic device fabrication

\subsubsection{Photomask design}

Fabrication of masters for soft lithography was achieved using a photoresist, which is exposed to ultraviolet light to define the shapes and positions of features. This requires the use of a photomask, which exposes selected regions of the photoresist to the UV source while shielding the remainder.

Photomasks used in microfabrication are usually made by coating chrome onto a transparent silica sheet. This allows very fine features to be defined with excellent contrast, but the masks are prohibitively expensive. Glass photomasks are more affordable, but still cost over $\$ 100$ per mask. These costs were avoided by simply printing the mask design onto an overhead transparency. The resolution achieved is inferior to standard photomask processes, but is adequate for producing features larger than $\sim 100 \mu \mathrm{m}$.

Photomask patterns were designed in Microsoft Visio [153], which was a low-cost alternative to more specialised CAD software. Because the designs are printed directly onto an overhead transparency, the use of Visio is appropriate for the task. An example of a photomask design produced in Visio, along with the resulting printout, is shown in Figure 23. The channels are defined by transparent regions in the photomask. This is because a negative photoresist was used, which is cross-linked by UV exposure. Photoresist obscured by the dark regions of the photomask will not cross-link, and will be dissolved during subsequent development. 

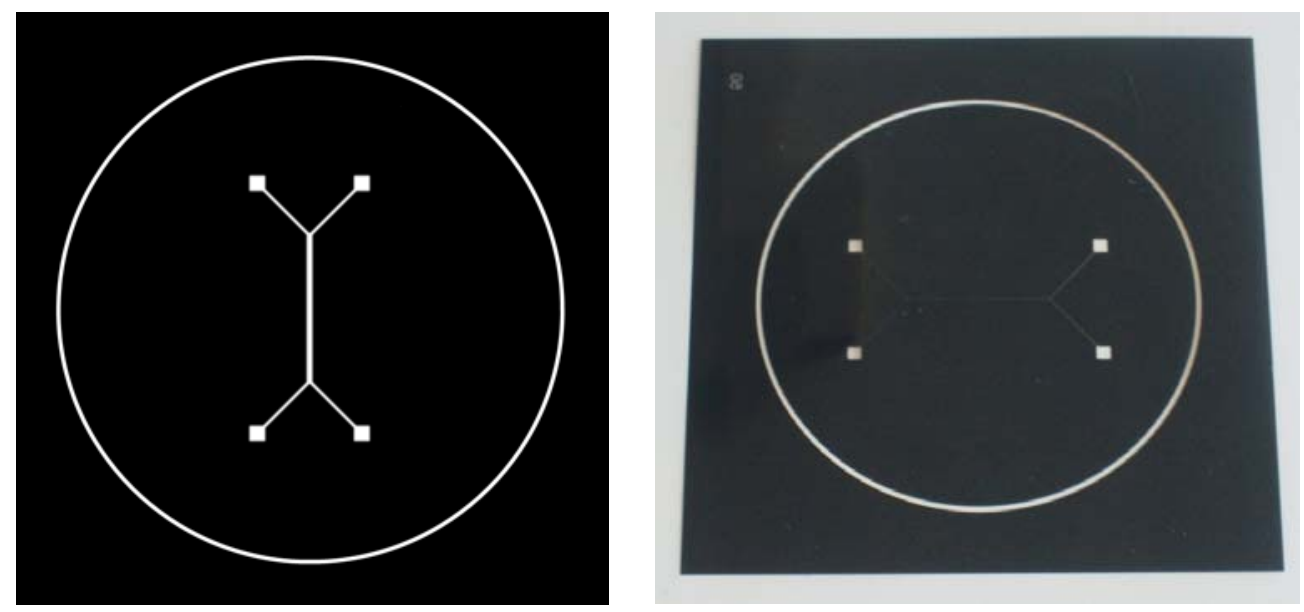

Figure 23: (Left) Photomask design created in Microsoft Visio. (Right) Resulting photomask after printing the design onto an overhead transparency. The ring is present to aid positioning of the photomask before exposure.

It can be seen that while the printout is a reasonable replica of the design, the contrast is somewhat reduced. This is not ideal, as a small amount of light may penetrate the mask and make the photoresist more difficult to develop. In order to maximise the contrast, the printer was set to 'High Resolution' mode, and the colour balance enhanced. Both measures increased the pixel density during printing, thus increasing the contrast. Photomasks produced under these conditions did not appear to allow unintended exposure of the photoresist.

\subsubsection{Fabrication of SU-8/PMMA masters}

The photolithographic procedure used to create microfluidic masters is summarised in Figure 24. A uniform layer of SU-8 photoresist is applied to a polymethyl methacrylate (PMMA) wafer by spin coating. The layer is partially cured by baking, before exposing the photoresist to UV light in the presence of a photomask. Photoresist exposed to UV light will rapidly cross-link upon further baking, preventing it from being removed when the wafer is immersed in a developer solution. The photoresist features retained after development will define the positions of the channels when the master is used as a mould. 


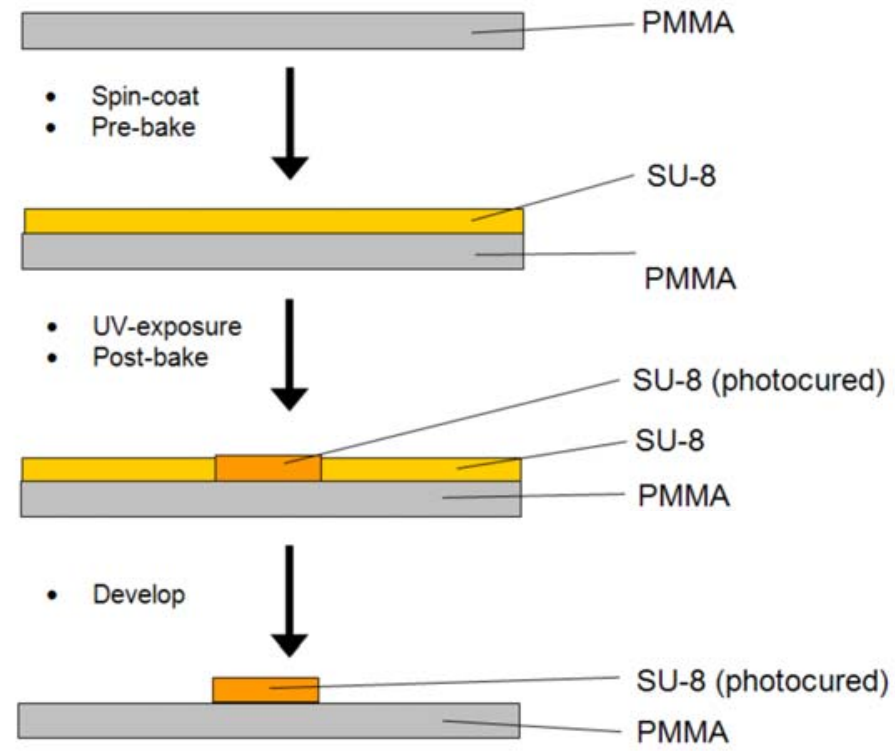

Figure 24: Fabrication of masters in the SU-8/PMMA system.

SU-8 is a commercially available photoresist produced by MicroChem [154] which is commonly used for microchannel fabrication [155,156]. As can be seen in Figure 25, each monomer contains eight epoxy rings. The acute bonding angles within the rings are energetically unfavoured, making the groups highly reactive. Triaryl sulfonium salt is added to the photoresist, and attacks the epoxy rings following UV-exposure [156]. Once opened, the epoxy groups allow polymerisation to occur. The large number of epoxy groups on each monomer enables substantial cross-linking. This gives rise to excellent mechanical and chemical resistance, facilitating the creation of high aspect ratio structures [157]. SU-8 is provided in a number of formulations of differing viscosity, allowing the thickness of the photoresist to be controlled. All work was performed using SU-8 2150, which should give a resist thickness of around $280 \mu \mathrm{m}$ when spun onto a silicon substrate at 2000 RPM [158]. Silicon was not used as the substrate in this project, meaning this thickness is merely a rough guide. 


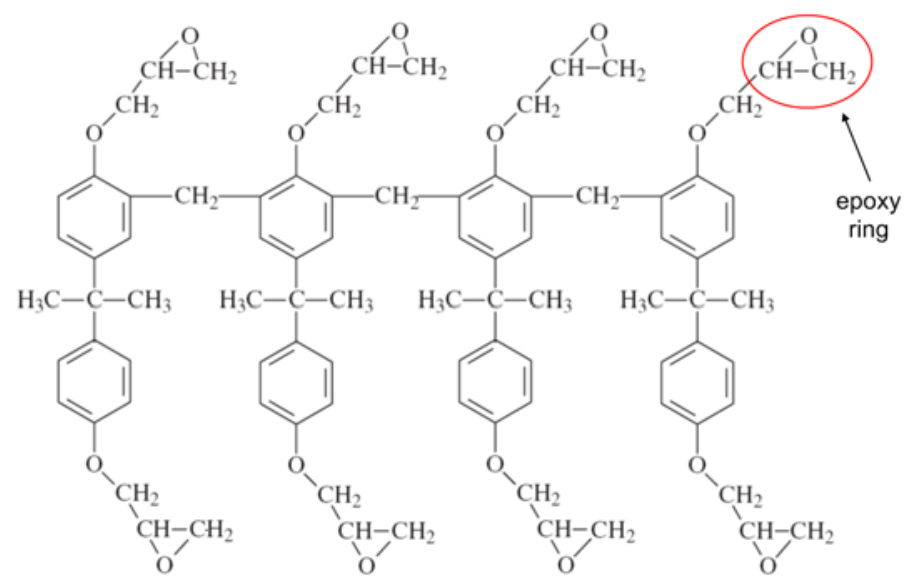

Figure 25: Structure of SU-8 photoresist monomers [90]. The monomer contains 8 epoxy rings, which allow for effective polymerisation.

Silicon is commonly used as the substrate for SU-8, largely due to its utility in microelectronics applications. However, in microfluidics fabrication the electronic properties are irrelevant, and silicon has a number of drawbacks. The most significant is that silicon is expensive to manufacture and machine into wafers. The surface energy of silicon is also very low, meaning that bonding between SU-8 and silicon is rather weak. The photoresist is therefore likely to detach from the silicon substrate when the master is being used.

In this project polymethyl methacrylate (PMMA or acrylic) was used in place of silicon. Bubendorfer et al. [90] recently detailed the use of polymethyl PMMA as a substrate, and found that SU-8 bonded very strongly to this material. Masters created using this method were therefore much more resistant to delamination than silicon. Material and machining costs for PMMA are also markedly lower than for silicon, helping to reduce fabrication costs. Acrylic does have some drawbacks, including a low glass transition temperature of $120^{\circ} \mathrm{C}$ [92], low thermal conductivity and poor solvent resistance. Lithographic procedures for SU-8 provided by the manufacturer also assume a silicon substrate, making it necessary to modify the standard protocol.

PMMA was supplied in sheets, which were then cut down into circular 2" or 3" wafers. Initially the wafers were machine-cut in the SCPS mechanical workshop. This left grease residues on the surface, making it necessary to clean the wafers using detergent 
and isopropanol. Even after thorough cleaning, the bond between the SU-8 and PMMA was often poor, causing channel features to detach from the wafer. More effective solvents such as butanone could not be used, as they rapidly damaged the PMMA surface. Major improvements were seen when a laser-cutting procedure was introduced. This avoided the need for grease to be applied to the PMMA, and even allowed the protective plastic to be left on. The new procedure greatly improved the cleanliness of the substrate, which in turn significantly improved the durability of the masters.

Fabrication of masters was performed in darkroom conditions, to minimise unwanted exposure of the photoresist to UV light. The procedure encompasses coating, baking, exposure and development steps, as outlined below:

\section{Coating}

A uniform layer of SU-8 was applied to the wafer using a WS-400-6NPP-LITE spincoater (Laurell Technologies [159]). Several millilitres of the photoresist was decanted onto the wafer, taking care to minimise bubbles. Best results were obtained by applying a slight excess of photoresist. The wafer was then spun at 500 RPM for 10 seconds to spread the photoresist, and then at 2000 RPM for 30 seconds to achieve the desired thickness. Small amounts of SU-8 would usually stick to the edges and underside of the wafer; these were removed using Edge Bead Remover (MicroChem [160]) and a lintfree cloth.

\section{Pre-bake}

In order to reduce the concentration of solvent present, the SU-8 is heated on a hotplate before UV exposure. This solidifies the photoresist layer, and increases the density of epoxy groups to enhance cross-linking. The wafer was heated at $75^{\circ} \mathrm{C}$ for 7 minutes, and then at $105^{\circ} \mathrm{C}$ for a further 45 minutes. This two-step process is aimed at minimising thermal stresses in the substrate. The wafer was covered during heating, to prevent dust particles falling into the SU-8. Once the pre-bake was completed, the wafer was allowed to cool to room temperature on the hotplate. 


\section{Exposure}

Exposure to UV light activates the triaryl sulfonium salt in the photoresist, causing it to open the epoxy rings on the SU-8 monomers and allow cross-linking. Using a photomask allows us to selectively expose the photoresist, with unexposed regions being removed during subsequent development. Exposure was performed using an AZ210 UV exposure unit (Mega Electronics [161], which has an output of 4-5 mW/ $\mathrm{cm}^{2}$. For an SU8 layer $150 \mu \mathrm{m}$ thick, a dosage of $260 \mathrm{~mJ} / \mathrm{cm}^{2}$ is recommended [158], which corresponds to an exposure time of roughly 60 seconds. The photoresist-coated wafer was manually aligned with the OHT photomask, and placed in the centre of the exposure unit. A layer of card was placed underneath the wafer to minimise reflections.

\section{Post-bake}

Following UV exposure, gradual polymerisation of the photoresist will occur. In order to accelerate this process, a second baking step was employed. The wafer was heated to $70^{\circ} \mathrm{C}$ for 5 minutes, before raising the temperature to $105^{\circ} \mathrm{C}$ for a further 20 minutes. The wafer was then left to cool down to room temperature on the hotplate before development.

\section{Development}

Unexposed photoresist was removed at room temperature using a chemical developer. A proprietary SU-8 developer (MicroChem [162]) was used for this process. The wafer was immersed in a Petri dish containing the developer, and the solution vigorously swirled over the wafer surface. The development time needed depends on the resist thickness, but in most cases four immersion cycles of two minutes length was sufficient. In between these cycles, developed SU-8 was rinsed off using isopropanol. It was common for some of the SU-8 to remain stuck to the wafer, and this was removed using gloved fingers.

\subsubsection{Replica moulding in PDMS}

Once a master has been fabricated, it can be used repeatedly to create microfluidic structures by moulding a polymer to the surface. In this project, moulding was performed in polydimethylsiloxane (PDMS). This material is one of the most developed polymers for microfluidics [163], as it possesses a number of unique and useful 
properties. Its low surface tension enables it to conform to fine features on moulds, making it ideal for soft lithography. It is transparent to wavelengths above $230 \mathrm{~nm}$, making it compatible with optical and spectroscopic detection methods. It is also nontoxic and gas-permeable, making it suitable for biological applications. The biggest drawback of PDMS is that many organic solvents can diffuse into the polymer network, causing reversible swelling [164].

As can be seen in Figure 26, PDMS consists of a Si-O backbone with two methyl groups on each $\mathrm{Si}$ atom. Weak bonding between the chains makes uncured PDMS a viscous liquid, whose unique flow properties make it able to be cast against a mould with submicron fidelity [163]. A curing agent can then be used to induce cross-linking, converting the material to an elastic solid. Once initiated, curing will proceed very gradually at room temperature. In most cases the sample is heated to accelerate the curing process.

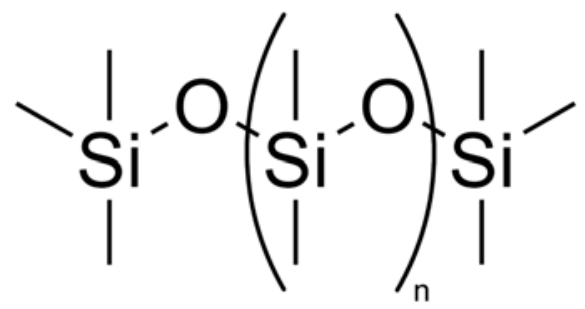

Figure 26: Molecular structure of polydimethylsiloxane (PDMS).

A Sylgard 184 Silicone Elastomer Kit (Dow Corning [165]) was used in the fabrication of all microfluidic devices. This kit consists of a PDMS base and a separate curing agent. Mixing the two components together will initiate cross-linking and eventually produce a solid PDMS elastomer. This two-component formulation extends the useful shelf-life, by preventing premature curing. The components should be mixed at a ratio of 10 parts base to 1 part curing agent (by mass) to obtain a flexible but solid material.

Mixing was performed in a disposable plastic cup, as the removal of cured PDMS from glassware is difficult. The components were mixed thoroughly by stirring for a few minutes with a glass stirring rod, to ensure homogeneous curing of the PMDS. Stirring inevitably leads to the introduction of bubbles, which must be removed. Bubbles present 
near the channels may cause leaks, and will also obscure visual observation of the channel. Bubbles were effectively removed by exposing the mixture to low vacuum using a rotary pump for approximately 30 minutes.

Once it has been mixed and degassed, the PDMS can be poured directly onto the surface of the SU-8 master. The PDMS was poured slowly to avoid introducing new bubbles. In order to prevent the liquid PDMS from flowing off the wafer, the edges were sealed off with aluminium foil. The wafer was then placed on a hotplate, and the PDMS was allowed to cure for 1-2 hours at a temperature of $75^{\circ} \mathrm{C}$. The actual time required depended on the thickness of the PDMS layer. Once curing had completed, the wafer was removed from the hotplate and allowed to cool to room temperature. The PDMS could then be peeled off the master and prepared for sealing.

Each device consisted of two layers of PDMS. The first was cast against a master containing the channel features. This layer was only a few millimetres thick, meaning the edges could be easily trimmed after moulding. A second sealing layer was cast against a flat PMMA wafer. This layer was around one centimetre thick, making it difficult to trim the edges cleanly. A casting mould was designed so that this layer could be cast with a cleaner edge, making subsequent trimming unnecessary. As pictured in Figure 27, the mould consisted of an aluminium block with a recess to hold the PMMA wafer. This recess was $9 \mathrm{~mm}$ deep, sufficient to allow a thick layer of PDMS to be poured onto the wafer. A $20 \mathrm{~mm}$-diameter hole was cut into the base, so that the wafer could be pushed out of the mould once the PDMS had cured.
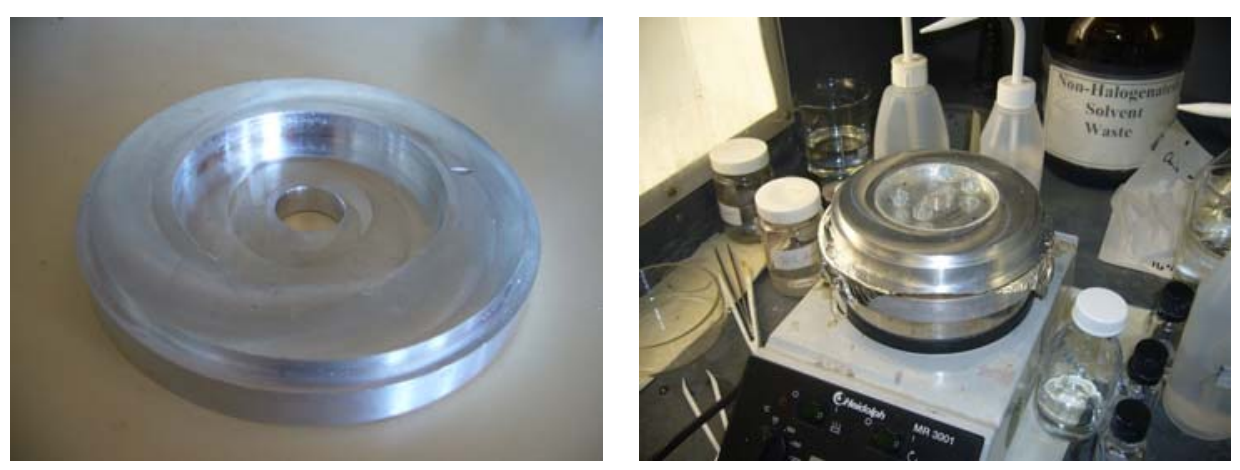

Figure 27: (Left) Aluminium casting mould used for curing PDMS layers with a defined edge. (Right) PDMS curing on a hotplate, using the mould. 


\subsubsection{Fluid connections}

A key aspect of any microfluidic device is the interface between the solution reservoirs (usually syringes) and the channels themselves. It is important that these interfaces are pressure-tight, in order to prevent leaks and bubbles forming. It is also desirable to minimise the volume of the connections, particularly if the solutions to be used are expensive or scarce. Finally, the connections should be inexpensive, so that they may be used on a disposable basis.

In this project $1 / 16 "$ barbed female luer adaptors (Cole Parmer [166]) were used as disposable fluid connectors. In most cases the connectors were made of polyvinyl chloride, although nylon versions were also used. A method was needed to attach the connectors to the PDMS. Adhesives were likely to be unreliable, as many do not stick well to hydrophobic surfaces like PDMS. The ability of PDMS to stretch and bend may also compromise the bond. The luer connectors were instead embedded directly into PDMS, relying on a conformal seal alone.

Conformal sealing was initially achieved by placing the connectors on a flat PMMA wafer, and then slowly pouring PDMS onto the wafer. After curing, the connectors would be rigidly embedded in a solid block of PDMS. A thin PDMS skin would typically form on the underside of the connectors, but this was readily trimmed away using a scalpel. The PDMS surrounding the connector could then be irreversibly bonded to the device by plasma oxidation (as described in Section 3.1.5). Holes were first

defined in the top layer of the microfluidic device, so that fluid could pass from the connectors into the channel. The resulting configuration is shown in Figure 28. 

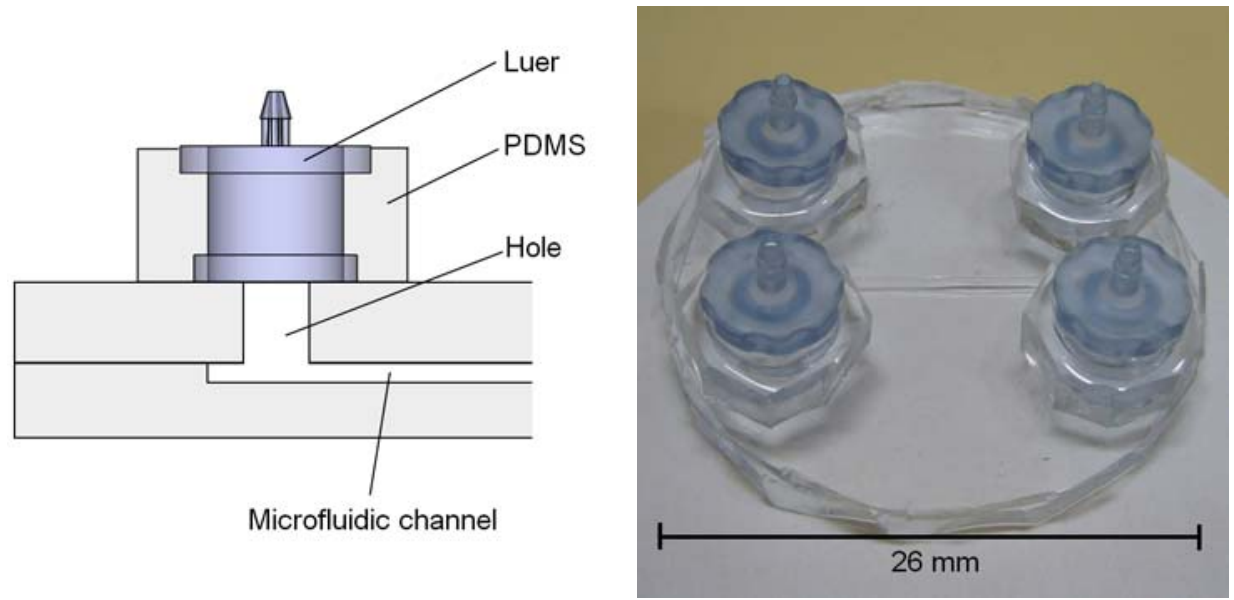

Figure 28: (Left) Fluid connection layout used in early microfluidic devices. (Right) An example of a microfluidic device with this connector layout

This layout was generally adequate, but had a number of drawbacks. The extra process steps increased the time needed to make each device. The connectors protruded significantly from the device surface, meaning they could obstruct the objective of a microscope when attempting to observe the channel. The dead volume was also unnecessarily large, due to the need to create a hole in the top PDMS layer. Finally, failure could potentially occur at either the PDMS-PDMS bond or the PDMS-luer bond. It is preferable to have a single interface, to reduce the number of potential points of failure in the device.

The final device designs still relied on conformal contact between the PDMS and the luer connectors. However, the connectors were embedded directly in the sealing layer of PDMS, as shown in Figure 29. In order to ensure the connectors were accurately placed, a felt-tip pen was used to mark the desired positions on the underside of the wafer. Best results were obtained when the thickness of the sealing layer was increased so that the connectors were embedded up to the base of the barb, as shown in the diagram. This prevented the connectors from being twisted out of place when handled, and also increased the overall rigidity of the device itself. 

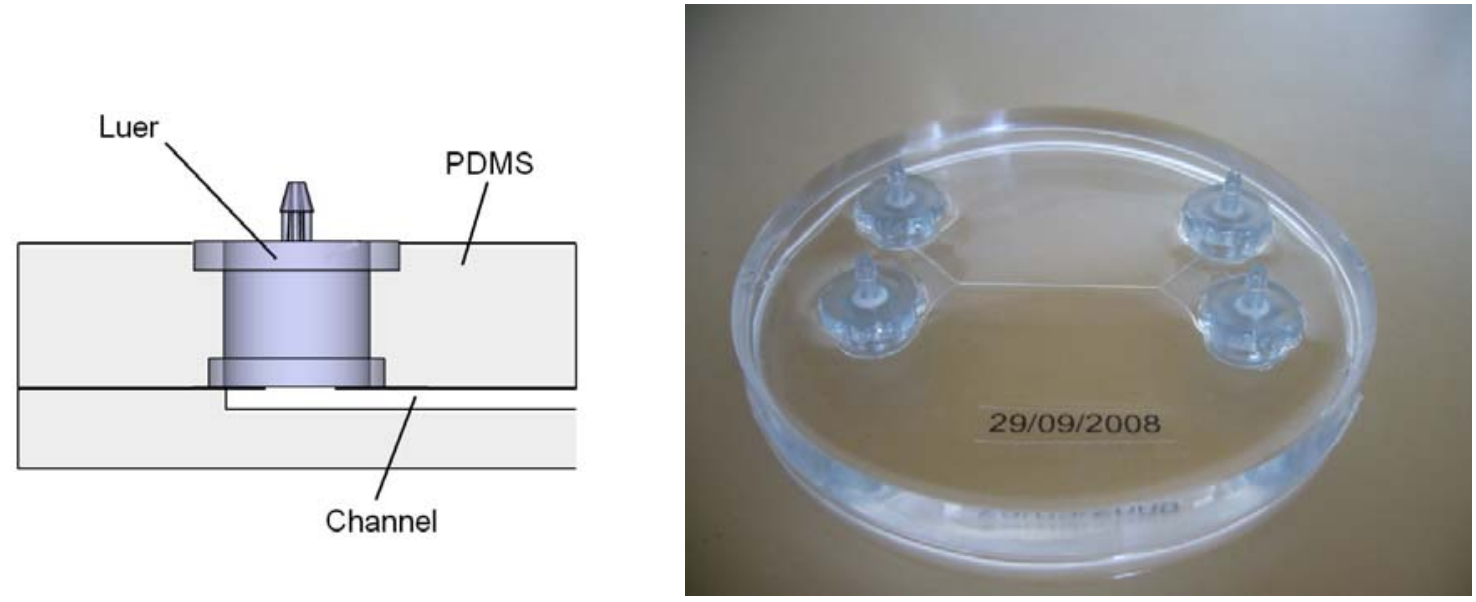

Figure 29: (Left) Fluid connection layout used in more recent microfluidic devices. (Right) An example of a microfluidic device with this connector layout.

This configuration reduced or eliminated all of the aforementioned drawbacks of the original layout. Dead volumes were reduced to that of the luer connectors themselves $(\sim 100 \mu \mathrm{L})$. Fabrication time was also reduced to that of making a device with no connectors at all. Finally, the connectors did not protrude above the surface of the device, making it easier to observe the channels using a microscope.

\subsubsection{Device sealing}

In order to create a finished device from the PDMS halves made by replica moulding, a reliable method to bond the pieces together was required. It is desirable that this method produces a reproducible bond, which is strong enough to withstand the pressure generated by the syringe pumps. It is also crucial that the technique does not negatively affect the channel itself.

The earliest bonding technique used was simple conformal contact between layers. The flexibility of the PDMS allows for extremely good contact between the two 'flat' surfaces, allowing for significant van der Waals attraction [167]. This sealing is reversible yet watertight, but will fail at pressures greater than $35 \mathrm{kPa}$ [151]. The use of adhesives produces bonds capable of withstanding pressures as high as $700 \mathrm{kPa}$ $[168,169]$. However, the risk of impregnating microchannels with the adhesive is a major problem. Partially curing the PDMS layers, before bringing them into contact, gives a strong but less reproducible bond [170]. Altering the ratio of PDMS base to 
cross-linking agent can produce a bond upon curing [99], which is watertight up to 500 $\mathrm{kPa}$ [168]. A drawback of this method is that the final product is softer and stickier than PDMS mixed at the standard ratio.

The most popular irreversible bonding technique is surface modification by plasma treatment. This was introduced by Duffy et al. [5], and involves exposing the PDMS layers in a plasma cleaner, before bringing the surfaces into conformal contact. A permanent bond will rapidly form, which can withstand pressures of $175-500 \mathrm{kPa}$ [168]. It is thought that exposure to the plasma exposes silanol groups at the surface, reducing the hydrophobicity of the surface. Over time the surface will recover, by undergoing polymerisation with the opposing surface [171]. The method depends on a vacuum to operate, increasing cost and reducing flexibility. Interest in plasma bonding has been refreshed by the introduction of a portable corona discharge system. This system works by ionising the air surrounding an electrode, producing a localised plasma that is stable at atmospheric pressure. Poorer control is achieved over the plasma dosage, but it has been found that the bond quality is relatively insensitive to the treatment conditions [172]. Bond strengths of 210-375 kPa may be achieved, making the system competitive with large-scale plasma cleaners [168]. Although the corona system is less effective at activating the surfaces, it allows surfaces to be brought into contact more quickly after treatment. Bonding occurs over several hours, meaning that the layers may be peeled off and repositioned if required.

In this project, a BD20-AC Corona Discharge Unit (Electro-Technic Products Inc, Chicago) was used to seal PDMS microfluidic devices by plasma oxidation. The procedure used was based on that used by Chooi [152]. The voltage knob was adjusted to produce a diffuse plasma (pictured in in Figure 30) around the 2" electrode. Care was taken to avoid excessive sparking from the electrode, which may degrade the PDMS surface. Surfaces to be treated were first cleaned, both to improve interfacial contact on bonding, and to prevent dust becoming trapped in the device channels. Initially the surfaces were cleaned using ethanol or isopropanol, before drying with a lint-free cloth. This process was later replaced by adhesive tape, which was found to be much more effective at lifting dust particles off the surface. Treatment using the corona unit was carried out immediately, with the electrode held at a distance of several millimetres from 
the surface. The duration of plasma treatment was variable, but was generally conducted for 20-30 seconds per side. The electrode was swept over the surface during this period, to ensure the dosage was even over the surface. Following treatment, the two halves of the microfluidic device were aligned by eye, and pressed together by hand. The device was left to stand overnight before use, to ensure that bonding was complete. Pressure was often applied by placing weights on top of the device, but it became apparent that this was unnecessary. PDMS conforms very effectively to minor imperfections on the opposing surface, meaning that simple contact was sufficient to allow a bond to form.
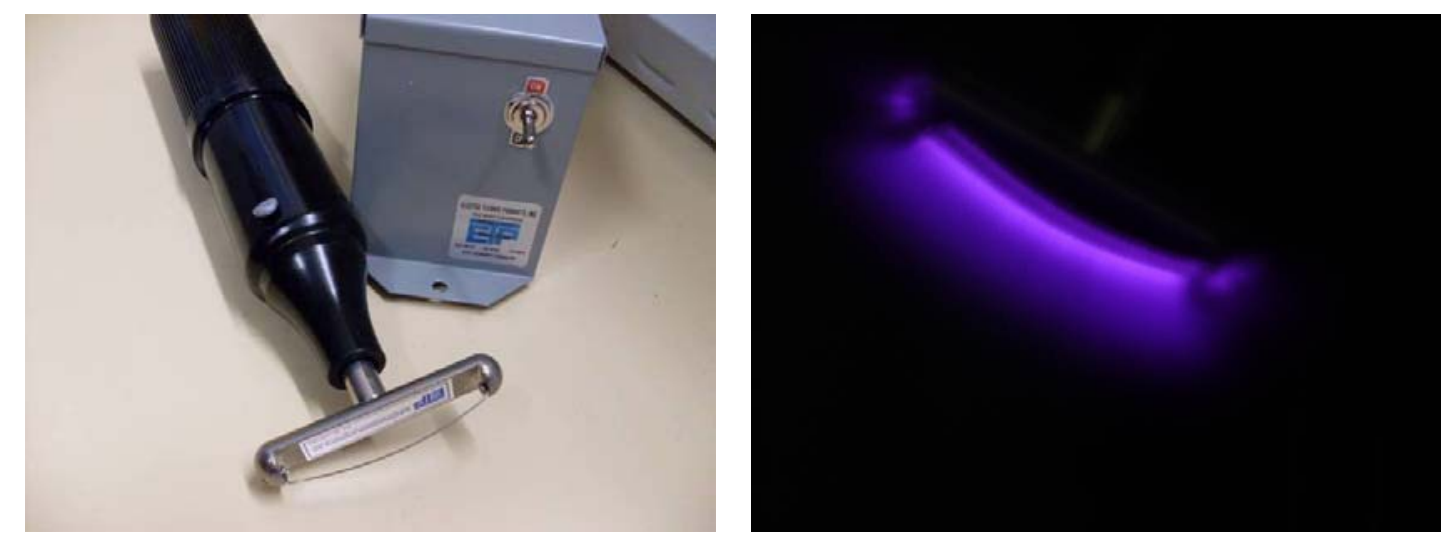

Figure 30: (Left) BD20-AC corona discharge unit used for plasma treatment of PDMS, showing electrode and power supply. (Right) Corona formed around 2" electrode during operation (photo taken using a 15 second exposure)

To investigate the effect of the corona on the PDMS surface, contact angle measurements were performed. Figure 31 shows a water droplet on a clean PDMS sample both before and after plasma treatment. The same sample 12 hours later is also shown. The droplet is slightly more spread out on the surface directly after the treatment, indicating a small reduction in the hydrophobicity of the surface. The contact angle was measured to within 5 degrees by image analysis, using the DropSnake [173] plug-in to the freeware program ImageJ [174]. Native PDMS showed a contact angle of $108^{\circ}$, which agreed within uncertainty with a value of $105^{\circ}$ reported by Haubert et al [172] for the same elastomer kit. After treatment the angle decreased to $83^{\circ}$, before eventually recovering to $104^{\circ}$. The reduction in contact angle was small compared to typical values of $30^{\circ}$, seen after treatment in a plasma cleaner [90]. However, the angle achieved is consistent with a small dose from the corona system $\left(<92^{\circ}\right)$ [172]. Longer exposure times are expected to lead to greater reductions in the contact angle, but also tend to 
generate a brittle silica layer on the surface [175], which can compromise contact between the surfaces.
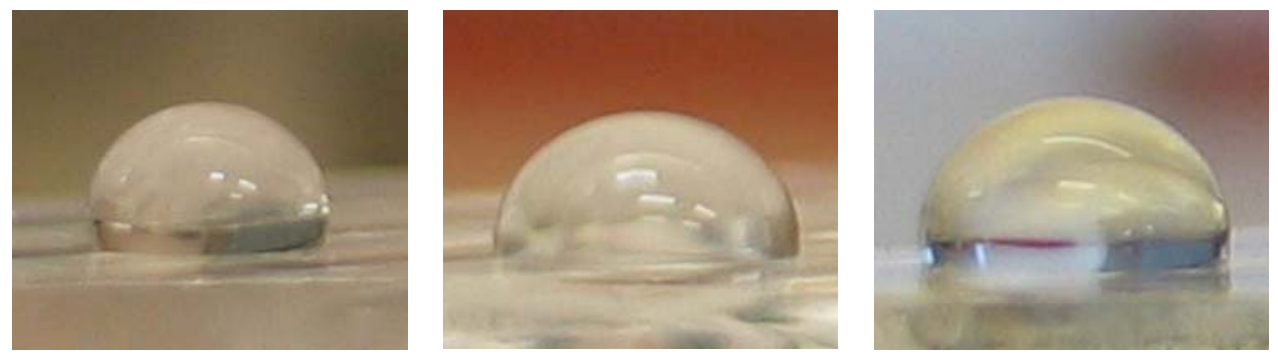

Figure 31: Water droplet on PDMS before corona treatment (left), immediately following treatment (centre) and 12 hours after treatment (right). The contact angle is slightly reduced after the treatment, but recovers to its original value within the 12 hour period.

Despite the relatively small change in contact angle resulting from the plasma treatment, assembled devices generally showed extremely strong bonding between the layers. It was impossible to peel the pieces apart without causing tearing in the PDMS itself. In some cases the bond would fail in certain regions, due to poor conformal contact between the surfaces. This was an issue when attempting to sandwich objects such as identification labels in between the layers, as these would often disrupt contact in the surrounding region and prevent bonding. If a meniscus formed at the edge of one of the PDMS layers, this could also pull the layers apart before a bond could form. Therefore it was important to first trim the layers, so that they were as flat as possible.

\subsubsection{Magnetic field gradient application}

A crucial aspect of a magnetic separation device is the application of a sufficiently large magnetic field gradient. It is this gradient which will allow particles to be captured or transferred from their original solution. In this project, three alternative separator designs were developed, each using a different approach to supplying this field gradient. The modifications to the device fabrication method will be discussed for each of these designs.

\section{Embedded magnet system}

The simplest method to supply a magnetic field is to place permanent magnets near the microfluidic channel. In most cases these are strong NdFeB magnets, which produce 
very large magnetic flux densities ( 500 $\mathrm{mT}$ [69]) at the pole surfaces. However, because permanent magnets tend to be much larger than the width of a microchannel, this does not translate into a large field gradient across the channel. In order to improve the field gradient that may be achieved, the size of the permanent magnets should be reduced. This does not reduce the surface flux density, but the field will decay over shorter length scales, producing a larger field gradient in the channel.

Miniature $\mathrm{NdFeB}$ cylindrical magnets were purchased in bulk from Frenergy [176]. As shown in Figure 32, these magnets have a diameter of $2 \mathrm{~mm}$ and are $1 \mathrm{~mm}$ thick. They are magnetised through the flat faces. The magnets cost only 10-20 cents each, making them suitable for use in disposable devices.
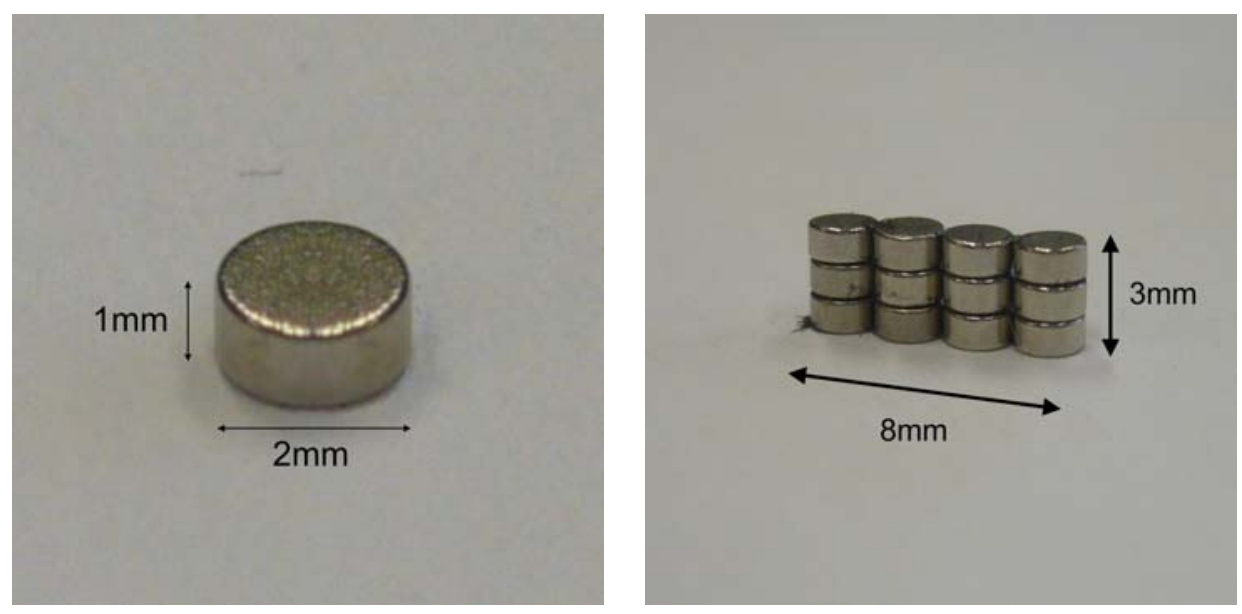

Figure 32: (Left) Miniature cylindrical magnets used to produce a magnetic field gradient in the embedded magnet system. The magnetic poles are on the flat faces. (Right) 12-magnet grid assembled from the magnets, for use in microfluidic devices.

The magnets were brought into close proximity with the channel by embedding them directly in the PDMS. The magnets were placed on the SU-8/PMMA master at the desired position, before pouring liquid PDMS onto the mould. In order to prevent them from rolling around on the wafer, the magnets were assembled into the grid pattern shown in Figure 32. This grid was rested flat on the wafer, with the magnetic poles orientated perpendicular to the channel length. The magnetisation directions of adjacent columns of magnets were antiparallel, as strong magnetic repulsion prevents a parallel configuration. 
The PDMS was poured slowly onto the mould, to avoid displacing the magnets from their intended position. Bubbles in the PDMS would often become trapped between the magnets and features on the master. These bubbles could cause the channel to leak, and so a needle was used to remove them. The PDMS was then allowed to cure on the wafer as normal, securing the magnets in place.

When using the corona discharge unit to treat the PDMS surfaces for sealing, the metallic surface of the magnets tended to draw sparks from the electrode. This did not appear to cause any damage to the magnets, but may reduce the dosage of plasma received by the surrounding PDMS. In spite of this however, the PDMS layers were still found to form a watertight seal around the magnets.

\section{Magnet-micrometer system}

Embedding magnets directly into the PDMS made it impossible to deactivate the field gradient applied across the microchannel to release trapped particles. An alternative device design was therefore sought for practical use. The ability to reduce or eliminate the magnetic field when required was achieved by positioning permanent magnets outside the device. The magnets could not be brought as close to the channel as when they were embedded, meaning the maximum field gradient was reduced, but the magnets could be readily moved.

A system was developed that allowed the distance between a small permanent magnet and the fluid channel to be accurately controlled. Referred to from here on as the magnet-micrometer system, the device is pictured in Figure 33. The microfluidic device rested on a Perspex stage, which had a micrometer installed in its base. Adjusting the micrometer allowed precise movement of a metal arm across the width of the stage. The magnet was then attached to the arm, allowing the magnetic field inside the microchannel to be varied in a controlled manner. The thickness of the upper PDMS layer however prevented the magnet from being moved near the channel. In order to allow closer positioning of the magnet, one of two alterations was made to the device. The first option was to mould the top PDMS layer in two pieces, leaving a notch across the width of the device. A thin $(<1 \mathrm{~mm})$ intermediate layer of PDMS was used to keep the channel sealed. However this only allowed the magnet to be swept from above the 
channel. It was found in preliminary testing that particles tended to be drawn towards the top of the channel, instead of across to one side. This approach was therefore abandoned, in favour of cutting a notch into the device after fabrication. The resulting notch extended through both the top and bottom PDMS layers, so that the magnet could approach the channel from side-on. A drawback of this method is that the notch must stop well short of the channel in order to prevent leaks.
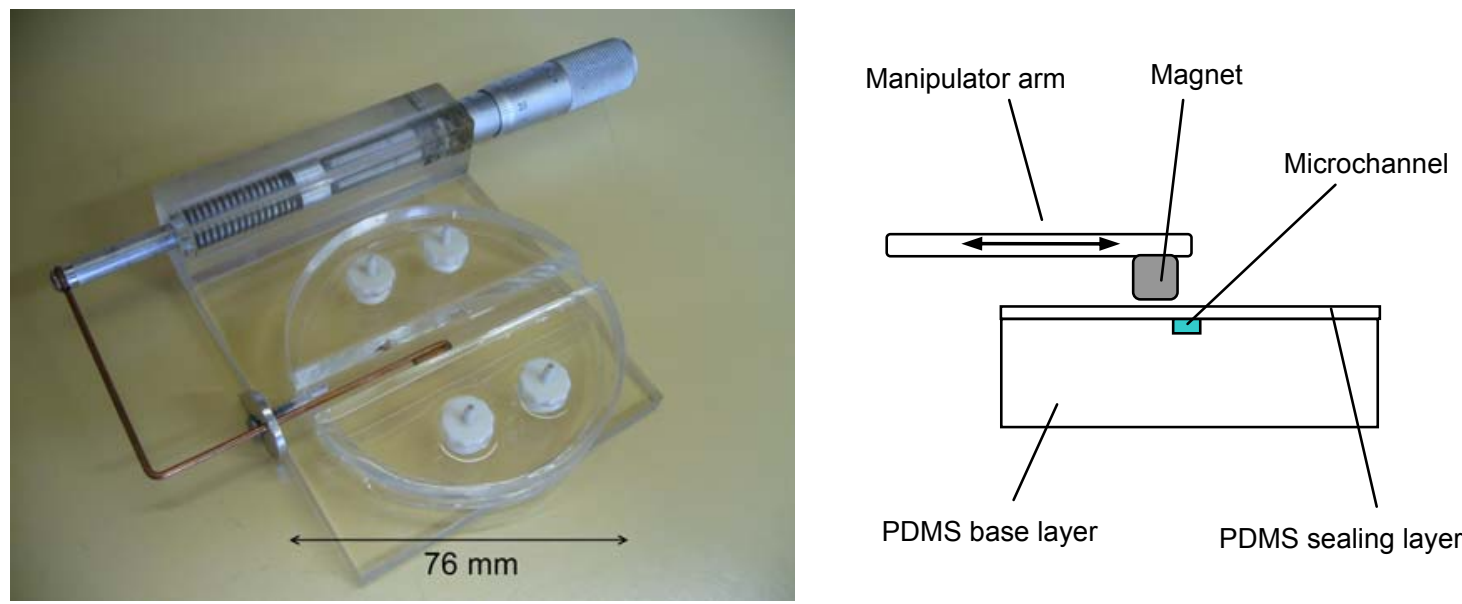

PDMS base layer

PDMS sealing layer
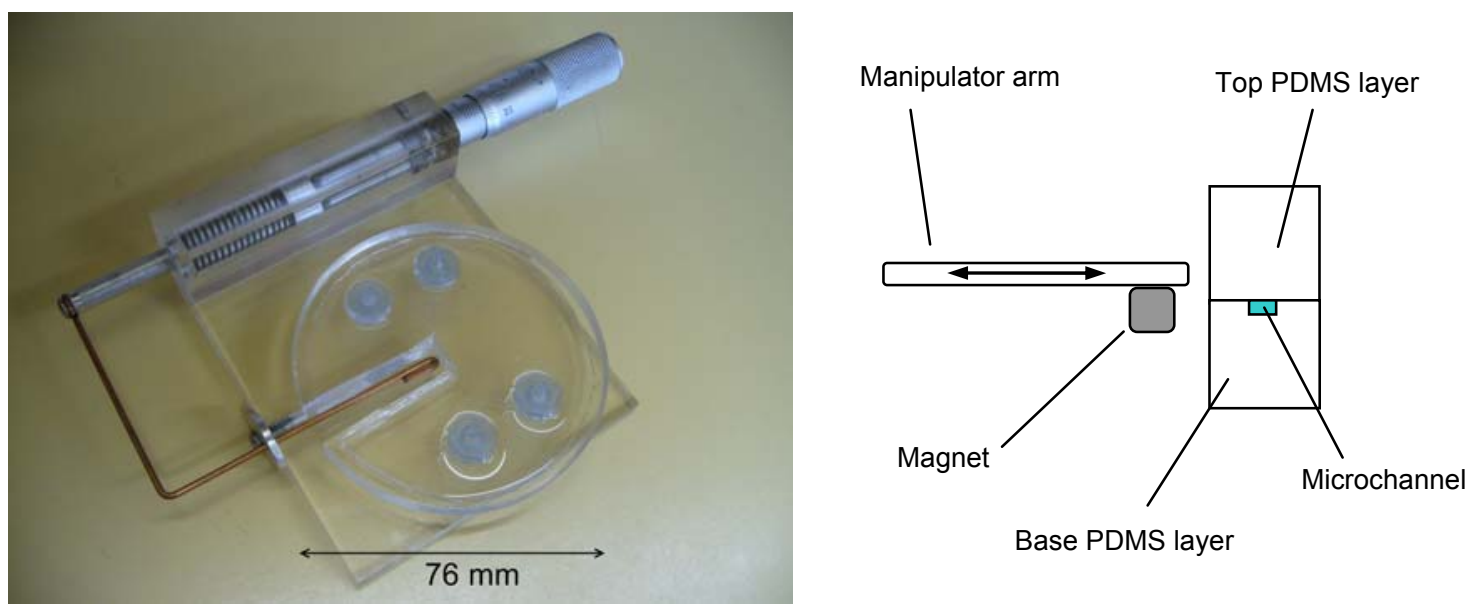

Figure 33: Microfluidic devices installed on a stage, allowing the relative position of a 3 mm wide cubic magnet to be manipulated using a micrometer. Two versions of the microfluidic device are shown, with schematic cross-sections.

Magnets similar to those used in the embedded magnet system were also purchased from Frenergy [176]. As shown in Figure 34 these magnets were cubic in shape, with a width of $3 \mathrm{~mm}$. A single magnet was attached directly to the metal arm of the micrometer setup, by relying on the magnetic attraction between the arm and the magnet. The magnet was orientated so that its pole was directed towards the side of the microfluidic 
channel. A possible drawback of this setup is that the metal arm may act as a flux guide, reducing the magnetic field strength inside the microchannel. Replacing the metal arm with a nonmagnetic material and securing the magnet with glue would overcome this limitation.

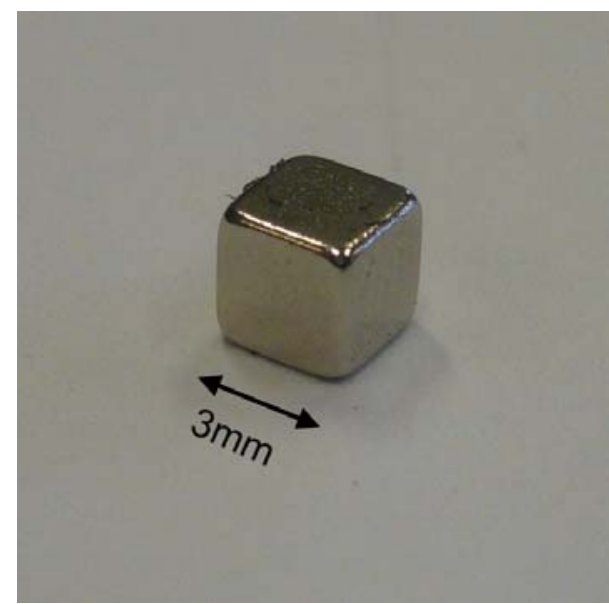

Figure 34: Miniature cubic magnets used to produce a magnetic field gradient in the magnet-micrometer system.

\section{Metglas system}

The third and final system developed made use of a soft magnetic material, which could be magnetised by an external magnetic field. This combines two advantages of the earlier systems. Firstly, the material can be permanently embedded in the device, in close proximity to the channel. At the same time the material will not produce a field gradient in the absence of an external field, allowing captured particles to be released when needed.

The soft magnetic material used in this project was an amorphous metal referred to as Metglas 2826MB [177]. This alloy contains over $80 \%$ iron and nickel by weight, making it strongly ferromagnetic [178]. However, the metal possesses a glass-like structure with no long-range order, which means it has a much lower coercivity than crystalline metals [179]. The magnetisation curve in Figure 35 confirms that the Metglas is magnetically soft. The width of the hysteresis loop is quite small, with a coercivity of just 15 gauss. The material should therefore not produce a significant field gradient in the fluid channel in the absence of an external field. Magnetic saturation is approached at relatively low fields, meaning permanent magnets can be used to activate the strips. 


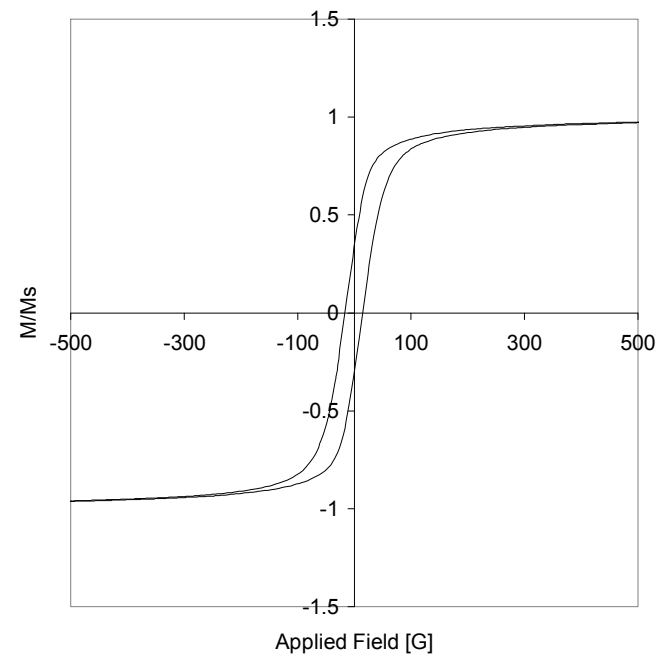

Figure 35: Magnetisation curve for Metglas 2826MB alloy, normalised to the saturation magnetisation of $0.8 \mathrm{~T}$. The small coercivity ( $\sim 15$ gauss) is typical of amorphous metals.

When designing the device, it was intended that it be used in a uniform field. This would mean that only the Metglas elements themselves would give rise to any translational forces on the particles. Unfortunately, no setup that could provide a sufficiently strong uniform field could be found. Smistrup et al. [130] used a metal frame that could direct the flux from an array of permanent magnets through a small area, producing a uniform field of 210 gauss. Using such an arrangement would be expected to improve the results obtained using the Metglas system, but was not used in the course of this project. Separation experiments were instead conducted using a $3 \mathrm{~cm}$ diameter $\mathrm{NdFeB}$ magnet to magnetise the Metglas strips.

As can be seen in Figure 36, the Metglas is supplied in thin ribbons several centimetres in width. The nominal thickness is just $30 \mu \mathrm{m}$ [177], making it an ideal replacement for thin magnetic layers formed using expensive processes such as metal evaporation and electroplating. The ribbon may be cut into the desired shape using scissors or a guillotine. In this project, rectangular strips $2-3 \mathrm{~mm}$ in width and $10-20 \mathrm{~mm}$ in length were typically used. 

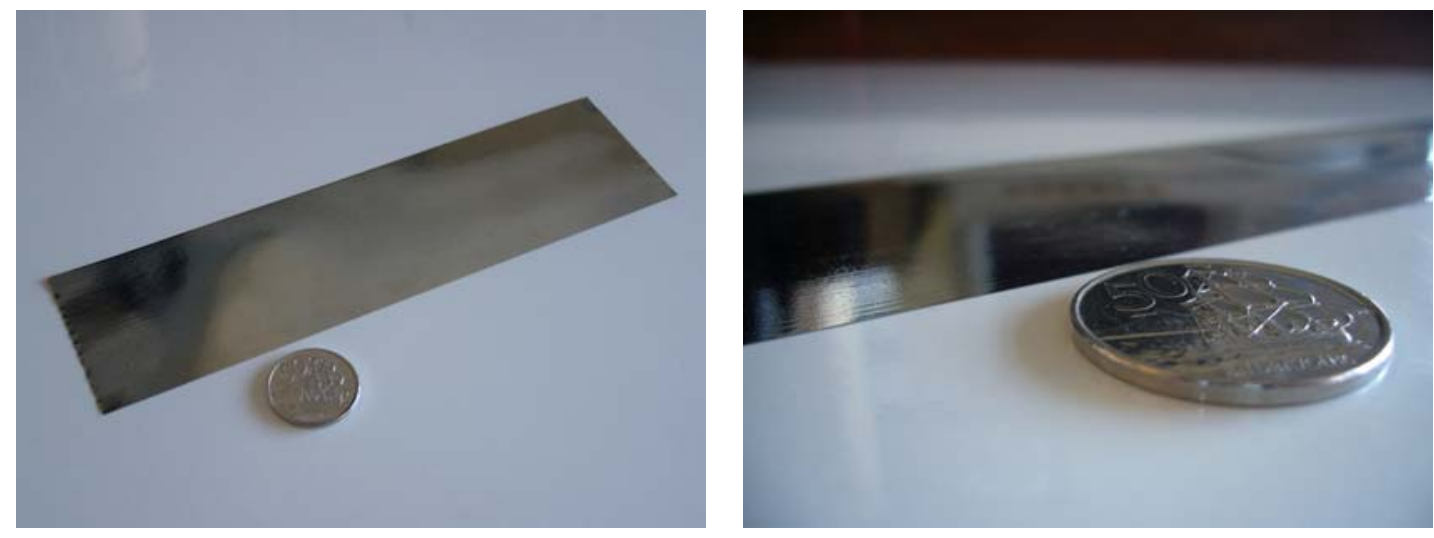

Figure 36: A ribbon of the amorphous metallic alloy Metglas 2828MB, used as a soft magnetic material for microfluidic magnetic separation. A 50 cent coin is included as a size reference.

Once the Metglas had been cut to the desired size, the strips were placed on the moulded PDMS replica, next to the fluid channel. The PDMS was then oxidised by corona discharge and sealed with a second oxidised PDMS layer. The Metglas strips caused sparking from the electrode, but this did not appear to damage the material. Unfortunately it was found that despite the minimal thickness of the Metglas, the two PDMS layers could not make sufficient contact to be properly sealed in the area around the strips. This led to a small bubble around each of the strips, which could cause leaks from the microchannel if the Metglas was placed too close.

In order to prevent the Metglas from compromising the bonding between the PDMS layers, a new master with recesses for the Metglas strips was designed. This also facilitated more reproducible placement of the strips. In order to determine the closest spacing between the recesses and the channel that would still keep the features separate, a master with multiple spacings was first created. The photomask design for this master is shown in Figure 37. The labels next to each recess indicate the width of the gap between it and the fluid channel. It is important to note that because features patterned in the SU-8 by UV exposure are somewhat larger than the size defined by the photomask, the actual spacing between features will be smaller than the labels suggest. A dye solution was driven through the channel of a PDMS replica of this master, and it was observed that the recesses remained fluid-tight provided they were defined to be at least $300 \mu \mathrm{m}$ from the channel on the photomask. 

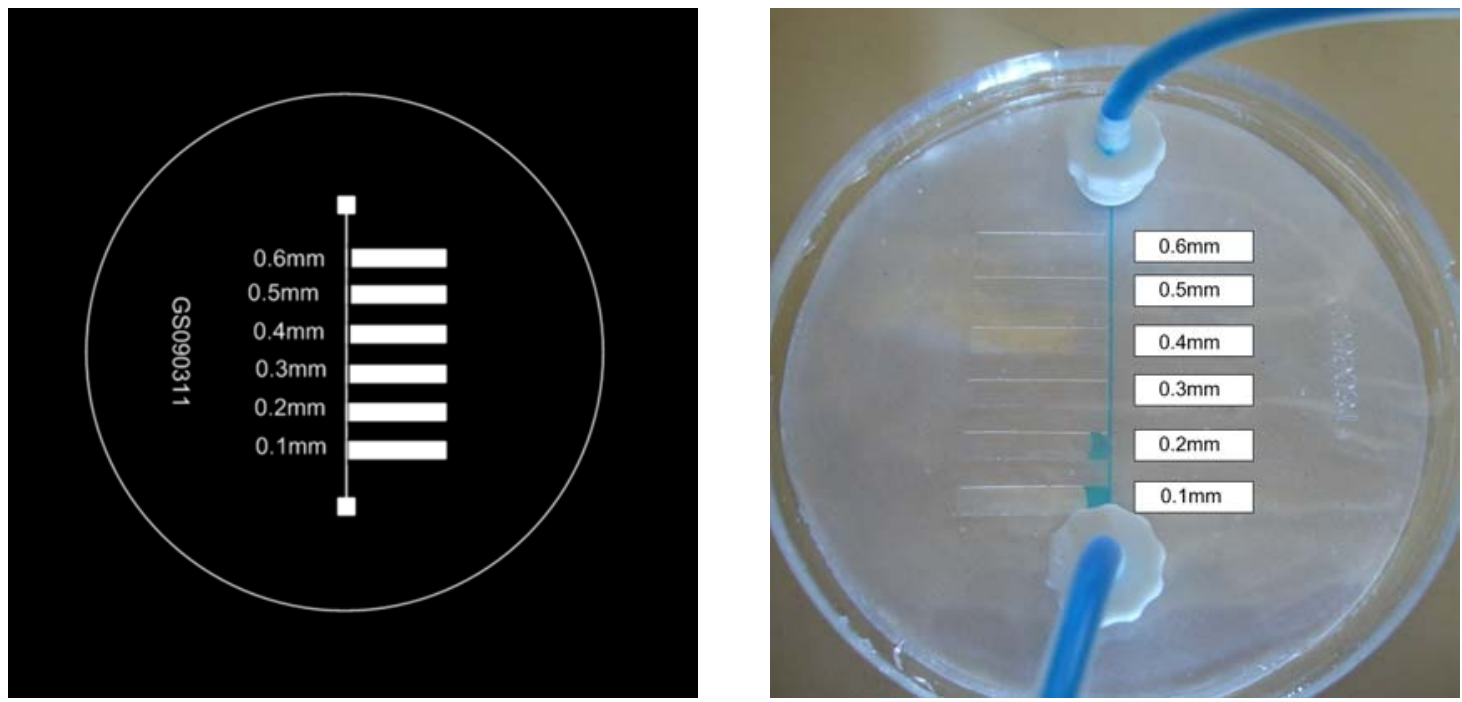

Figure 37: (Left) Photomask design for the master used to determine the optimal spacing between the recesses and the channel. (Right) Dye was forced through the channel of a PDMS replica. Leaking did not occur for spacings (on the photomask) of $300 \mu \mathrm{m}$ or greater.

Once the minimum feature spacing was determined, a master with multiple recesses for holding the Metglas strips was created. Displayed in Figure 38, this master contained five recesses, each spaced $300 \mu \mathrm{m}$ from the channel. As will be discussed in Section 4.3.7, using this photomask actually produced recesses spaced $200 \mu \mathrm{m}$ from the channel, due to the properties of the UV source used. Final magnetic separation devices were then produced in PDMS using the same process discussed earlier. It was found that the introduction of recesses for the Metglas greatly improved the sealing of the PDMS, allowing the Metglas to be brought closer to the channel than was previously possible. 

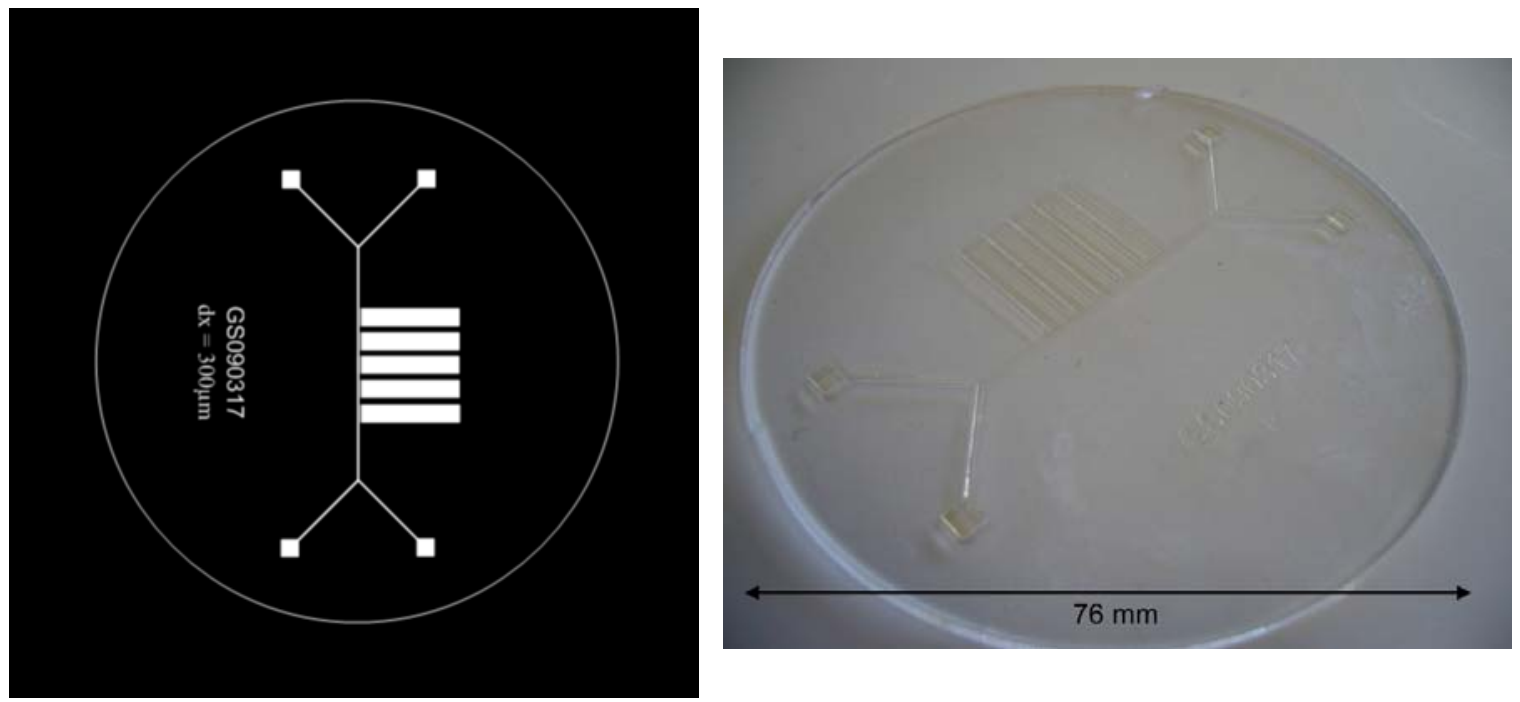

Figure 38: (Left) Photomask design used for fabrication of the Metglas magnetic separation device. (Right) SU-8/PMMA master produced using the photomask.

\subsection{Synthesis of magnetic nanoparticles}

Iron carbide, iron and iron oxide magnetic nanoparticles were synthesised using a solution phase synthesis method developed by Cheong [39], which is described in this section. This is followed by an explanation of the methods used to recover particles from the solution following the reaction. It was necessary to recap the nanoparticles to enable dispersion in aqueous solution, so that they could be used for experiments in PDMS devices. The section closes with a description of the techniques used to achieve this.

\subsubsection{Thermal decomposition}

As discussed in Section 2.2.1, preparation of magnetic nanoparticles in solution involves a gradual approach to saturation of the solution. Precipitation of the solute will then commence through the nucleation of fine particles. Ideally, only a single nucleation event occurs, and particle growth continues through diffusion of material to the particle surfaces. This separation of particle nucleation and growth allows for the synthesis of monodisperse nanoparticles.

In this project, the iron complex $\mathrm{Fe}\left(\mathrm{C}_{5} \mathrm{H}_{7}\right)_{2}$ was used as the organometallic precursor. The compound is similar to ferrocene, which has the molecular formula $\mathrm{Fe}\left(\mathrm{C}_{5} \mathrm{H}_{5}\right)_{2}$. However, the presence of extra hydrogen atoms on the molecule gives rise to a less stable structure, which will decompose at lower temperatures. As the material breaks 
down, it gradually increases the iron concentration in the solution. The complex was supplied in powder form by Cheong [39], and was stored under refrigeration in an evacuated bottle to protect it from oxidation.

The reaction must be carried out in an organic solvent, to suppress oxidation of the particles during growth. Toluene was initially used, due to its low polarity and cost. Toluene also has a reasonably high boiling point of $116^{\circ} \mathrm{C}$, allowing high reaction temperatures to be used. Mesitylene was later used in place of toluene, due to its higher boiling point of $165^{\circ} \mathrm{C}$. This allowed the reaction temperature to be further increased. As Figure 39 shows, the structures of toluene and mesitylene are very similar, meaning the reaction environment should remain otherwise unchanged.

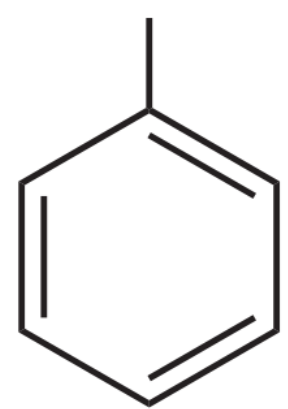

toluene

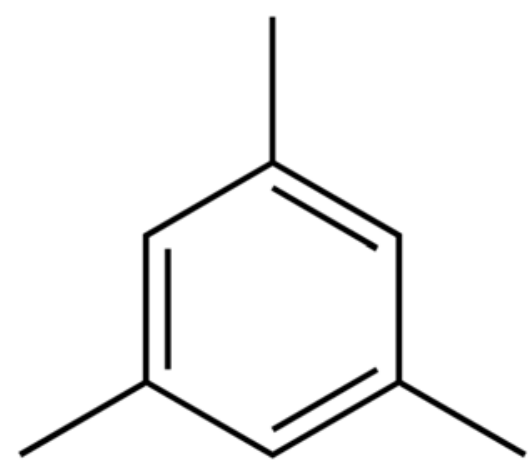

mesitylene

Figure 39: Molecular structure of toluene and mesitylene. Mesitylene has two extra methyl groups attached to the benzene ring, which give rise to a higher boiling point.

Oleylamine is added to the reaction mixture as a surfactant. The surfactant molecules 'cap' the surface of the particles during growth. This prevents aggregation, mediates the diffusion of material to the particle surface during growth, and protects the end product against oxidation.

The quantities of reactants used in most cases are listed in Table 2. The ratio of surfactant to solvent is of particular importance. When altering the solution volume, this ratio was kept constant. Each reactant was flushed with flowing nitrogen gas for approximately 20 minutes to displace oxygen. The solution was then mixed in a sample vial, before being transferred to the Fisher-Porter (FP) bottle pictured in Figure 40. The bottle featured gas fittings to allow evacuation or pressurisation of the vessel. A 
secondary vial was used to hold the solution if very small volumes were used, but in most cases the solution was poured directly into the bottle.

\begin{tabular}{|c|c|c|}
\hline Role & Reactant & Standard quantity \\
\hline Fe source & $\mathrm{Fe}\left(\mathrm{C}_{5} \mathrm{H}_{7}\right)_{2}$ & $0.8 \mathrm{~g}$ \\
\hline Surfactant & Oleylamine & $4 \mathrm{~mL}$ \\
\hline Solvent & Mesitylene & $16 \mathrm{~mL}$ \\
\hline
\end{tabular}

Table 2: Standard quantities of reactants used in the synthesis of iron carbide/iron/iron oxide magnetic nanoparticles

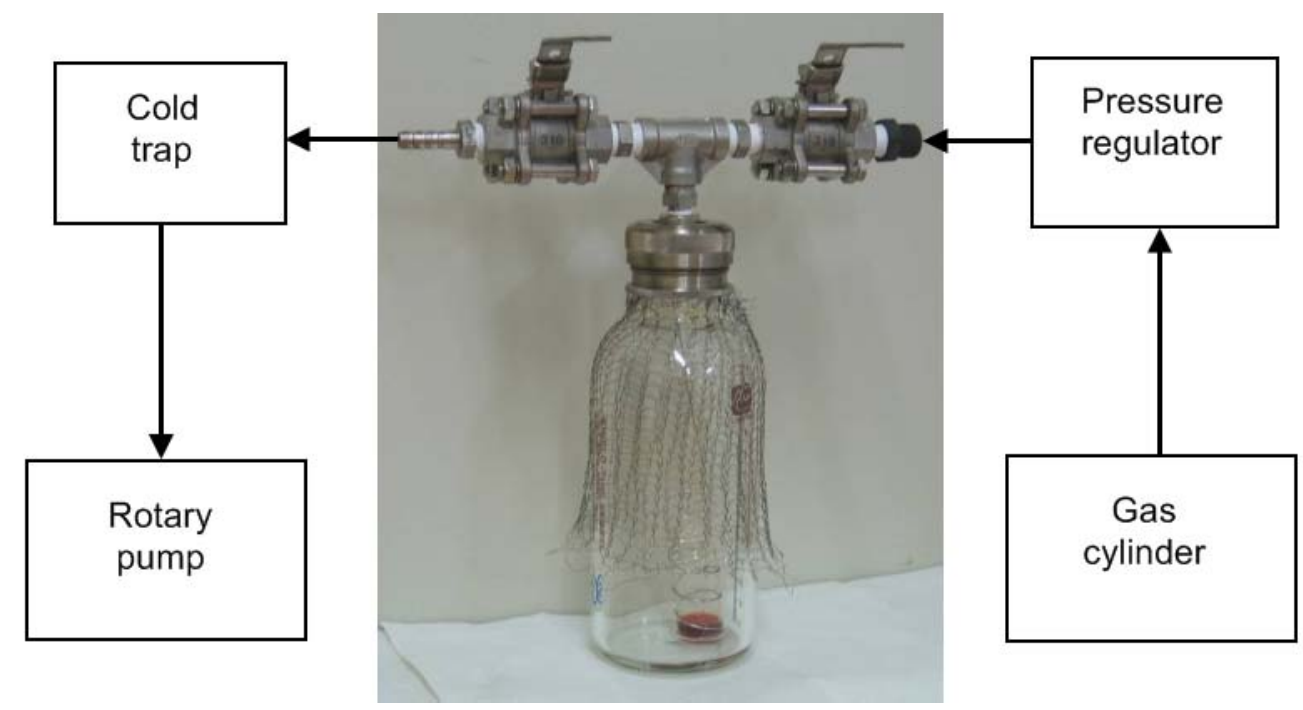

Figure 40: Fischer-Porter reaction vessel, with gas fittings to allow connection to a gas source and vacuum pump. The arrangement of components to allow evacuation and introduction of hydrogen gas is indicated.

The reaction must be conducted in a reducing atmosphere, and hence the FP bottle was connected to a hydrogen source and rotary pump, as represented in Figure 40. A regulator connected to the hydrogen cylinder allowed the incoming gas pressure to be controlled. The rotary pump was connected via a cold trap, to prevent organic vapours damaging the pump. The bottle was evacuated, and then filled with hydrogen to a gauge pressure of $100 \mathrm{kPa}$. This process was repeated 3 times to ensure all oxygen had been flushed out of the vessel. The bottle was then sealed and transferred to a furnace. The reaction was left to proceed at a temperature of $130^{\circ} \mathrm{C}$ for a period of 50 hours. 
Once the reaction had run to completion, the FP bottle was removed from the furnace and allowed to cool down to room temperature before opening. The solution was extracted using a pipette, and the bottle rinsed with toluene to collect any residues. The solution that was collected contained excess surfactant and unconsumed reagent, making it necessary to purify the solution.

\subsubsection{Manual purification}

The reaction described in Section 3.2.1 is expected to produce a small quantity of iron carbide and iron nanoparticles, and a larger quantity of smaller iron oxide nanoparticles. Although the reaction solvents were flushed with nitrogen, enough oxygen still remained dissolved to oxidise the smaller particles. It was desirable to obtain pure samples of each particle type from the reaction product.

The iron and iron carbide particles are expected to be more strongly magnetic than the iron oxide, and therefore magnetic separation was employed for their collection. A large Alnico magnet was placed next to the vial containing the solution. As shown in Figure 41 , over a period of a few minutes the iron and iron carbide particles were drawn towards the sidewall, while the rest of the material remained dispersed in the solution. The solution could then be removed using a pipette, and the captured particles redispersed in pure toluene. This process was usually repeated twice, as further washes removed too much surfactant and caused permanent aggregation to occur. The particles were then either left in solution, or the solvent was allowed to evaporate leaving a powder. 

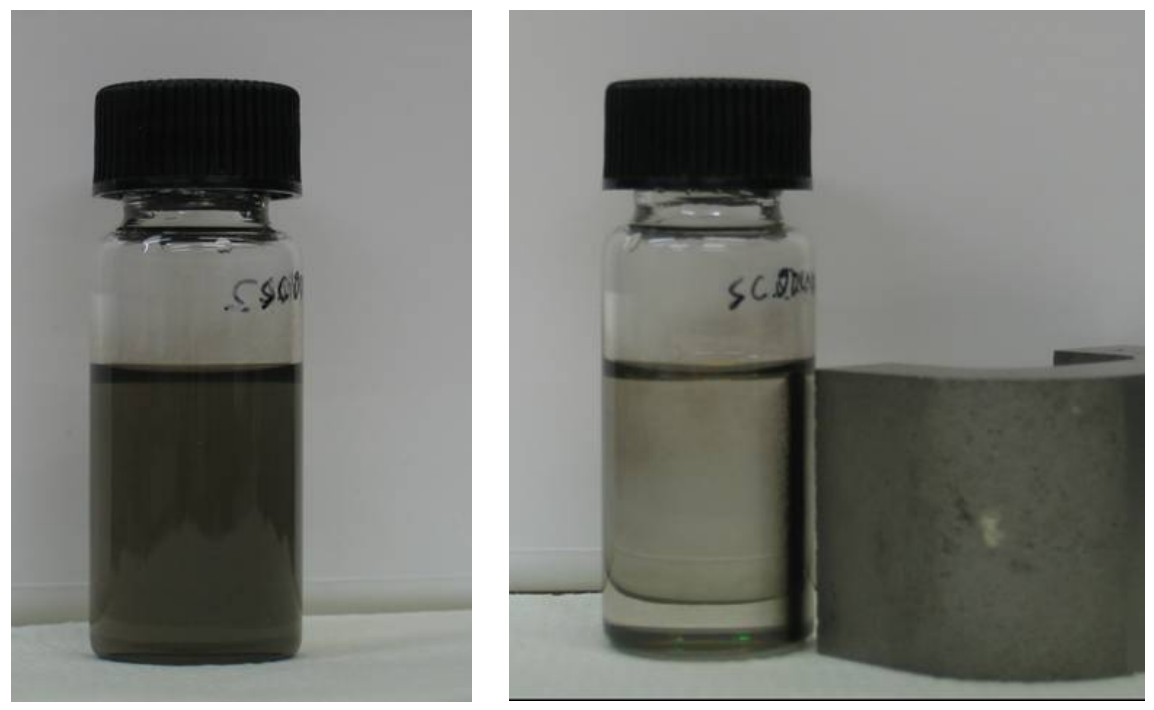

Figure 41: Capture of iron and iron carbide magnetic nanoparticles against the sidewall of a sample vial using a magnet. Here the solution is already purified, and the solvent therefore becomes transparent.

Once the iron and iron carbide particles had been removed, the iron oxide particles were collected by centrifugation. A centrifuge increases the effective gravitational force on the sample, and hence will cause the dense iron oxide nanoparticles to rapidly settle at the bottom of the sample holder. Sedimentation was further promoted by dispersing the particles in a 1:1 mixture of toluene and methanol. The reduced solubility of the capping agent in methanol reduces the stability of the dispersion. Centrifugation was performed at 4000 RPM for 10 minutes, and the solution immediately decanted. The particles were then redispersed in a toluene/methanol mixture, and the process repeated to remove excess capping agent. A total of three steps was usually found be adequate. Further purification risked removing too much surfactant and promoting particle aggregation. The particles were either left in solution, or the solvent evaporated to yield a powder.

During both forms of purification, nitrogen gas was bubbled through all solvents used to reduce their oxygen content. This was of particular importance when handling iron and iron carbide nanoparticles, as they are only expected to be oxidised near their surface. Further oxidation may therefore occur if steps are not taken to prevent it. The much smaller iron oxide particles are oxidised through their full volume, and therefore protection against further oxidation is relatively unimportant. The iron oxide may gradually change from $\mathrm{Fe}_{3} \mathrm{O}_{4}$ or $\gamma-\mathrm{Fe}_{2} \mathrm{O}_{3}$ to the magnetically weak $\alpha-\mathrm{Fe}_{2} \mathrm{O}_{3}$, but the spinel phases are expected to remain stable for a number of years [180]. 


\subsubsection{Recapping of particles for use in aqueous solvents}

Iron and iron carbide nanoparticles were recapped using a ligand-exchange process developed by Ferguson [181]. Dimercaptosuccinic acid (DMSA) was used to replace oleylamine as the capping agent, due to its stability in aqueous solution and its biocompatibility. Figure 42 shows the structure of DMSA, including the carboxyl groups that coordinate to the nanoparticle surface.

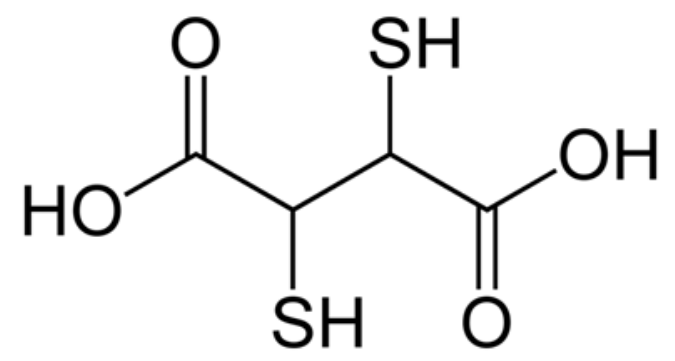

Figure 42: Molecular structure of dimercaptosuccinic acid (DMSA)

DMSA was supplied in powder form, and was weighed out in a 1:2 mass ratio to the iron carbide powder. The DMSA was dissolved in $5 \mathrm{~mL}$ of dimethyl sulfoxide (DMSO), while the iron carbide was dispersed in hexane. Hexane is only slightly soluble in DMSO, so the hexane formed a layer on top of the DMSO when the liquids were mixed. A single drop of triethylamine (TEA) was added to the mixture to raise the $\mathrm{pH}$ to approximately 9. Under basic conditions the carboxyl groups deprotonate, producing localised negative charges. The electrostatic repulsion produced by these charges help protect against particle aggregation.

In any surfactant-stabilised dispersion, capping molecules constantly detach from the surface, and are replaced with others in the solution. At the interface between the hexane and DMSO, DMSA molecules can bind to the particle surfaces as the oleylamine molecules detach. These particles can then migrate across the boundary into the DMSO. The bilayer mixture was sonicated for 6-7 hours to promote this process. Upon completion, most of the particles had left the hexane layer and occupied the DMSO layer.

Following ligand exchange, the hexane layer was removed by pipette. This was replaced with an equal volume of ethanol, which is miscible with DMSO. The particles were 
extracted using a magnet, and redispersed in $10 \mathrm{~mL}$ of ethanol, thereby cleaning away the DMSO. Another drop of TEA was added to restore the high $\mathrm{pH}$, and the solution was then sonicated overnight.

Using the described process yielded particles which were readily dispersed in ethanol or distilled water. However, many of the particles formed aggregates, which caused the solution to sediment within one hour. This is thought to have occurred due to excessive dilution of the solution, which reduced the ratio of surfactant molecules to particles. For this reason, particles prepared by Ferguson [181] using the same process were instead used for magnetic separation experiments.

\subsection{Flow control}

A critical component of almost all microfluidic systems is the manipulation of the fluid flow in the channels of the device. This is usually achieved using an external pumping mechanism such as a syringe pump. This section describes the pumping setup used in characterising the flow in microfluidic channels, as well as in magnetic separation experiments. The use of pressure-driven flow will place physical strain on any microfluidic device, and hence a basic mathematical treatment of this pressure build-up is also presented.

\subsubsection{Experimental setup}

Pressure-driven flow was used in all experiments, due to its availability and simplicity. As discussed in Section 2.3.5, a pump is used to create a pressure gradient down the length of the channel. The fluid will then be propelled from regions of high to low pressure. In this project, a pair of NE-1000 syringe pumps (New Era Pump Systems [182]) was used. A photo of the pumps is shown in Figure 43, without syringes installed. The pumps feature a front-end panel, which enable the user to specify the desired flow rate and the syringe diameter. This information is used to automatically determine the driving rate of the pump, which forces the plunger into the syringe. Control is also granted over the total volume dispensed, and whether to dispense or withdraw solution. The range of flow rates that can be produced using the pumps is dependent on the syringe diameter. For $1 \mathrm{~mL}$ syringes, which were used in most experiments, flow rates as low as $0.01 \mu \mathrm{L} / \mathrm{min}$ and as high as $880 \mu \mathrm{L} / \mathrm{min}$ could be achieved. 


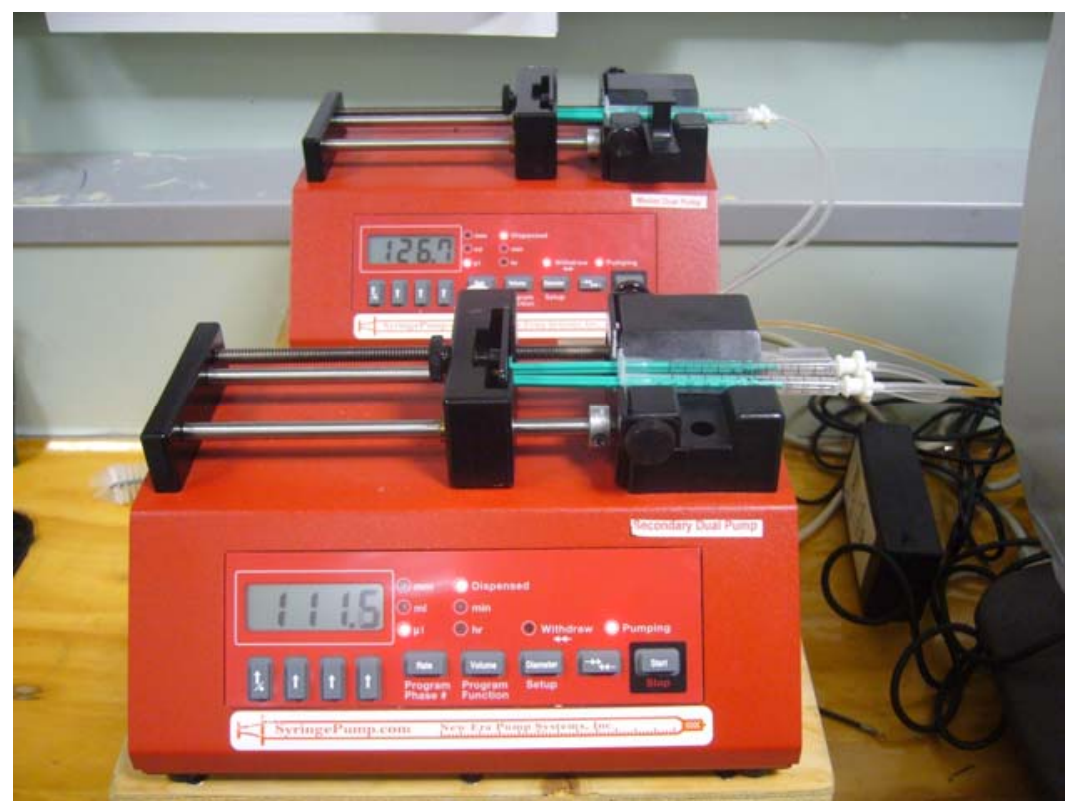

Figure 43: NE-1000 syringe pumps used for producing fluid flow in microfluidic devices.

In most experiments, connections between the syringes and the PDMS device were made using 1/16" diameter silicone C-Flex tubing (Cole-Parmer [183]). This tubing was selected as it was relatively inexpensive, and had properties suitable for most experiments. It is highly flexible, and capable of withstanding pressures of over $150 \mathrm{kPa}$. It is also transparent, allowing the fluid to be observed directly. Durability was also good, allowing stock to be used for multiple experiments. Tubing was connected to both the syringes and the device using 1/16" barbed luer adaptors. PTFE tubing (Cole-Parmer [184]), was briefly used when working with organic solvents, to which the silicone tubing shows poor resistance. However its inflexibility made positioning equipment difficult, and often damaged the luer adaptors.

In early experiments, syringes feeding the two inlets into the microfluidic device were installed on separate syringe pumps. Tubing was then run from the device outlets into collection or waste vessels. This allowed the flow rates delivered into each inlet to be controlled independently. However, this caused problems if bubbles entered the channel. Any bubbles that formed would be swept down the central channel, but would then become pinned in one of the two outlet channels and produce an obstruction. Fluid that would normally have exited at this outlet would instead exit at the opposite, 
unobstructed outlet. While it is possible to minimise the formation of bubbles, it is unlikely that they will be eliminated entirely. This prompted the use of a new configuration, in which one syringe pump was used to drive both input syringes. The second pump was used to withdraw the solution at the outlets into collection syringes at the same flow rate. This sacrificed the ability to set different flow rates for the buffer and solution streams, but rapidly cleared any blockages of the channels by bubbles. An additional benefit is that the withdrawal of solution at the outlets will supply a negative pressure, meaning the channels only experience atmospheric pressure during steadystate flow. This configuration, shown in Figure 44, was used in all magnetic separation experiments where fractions were needed for quantitative analysis.

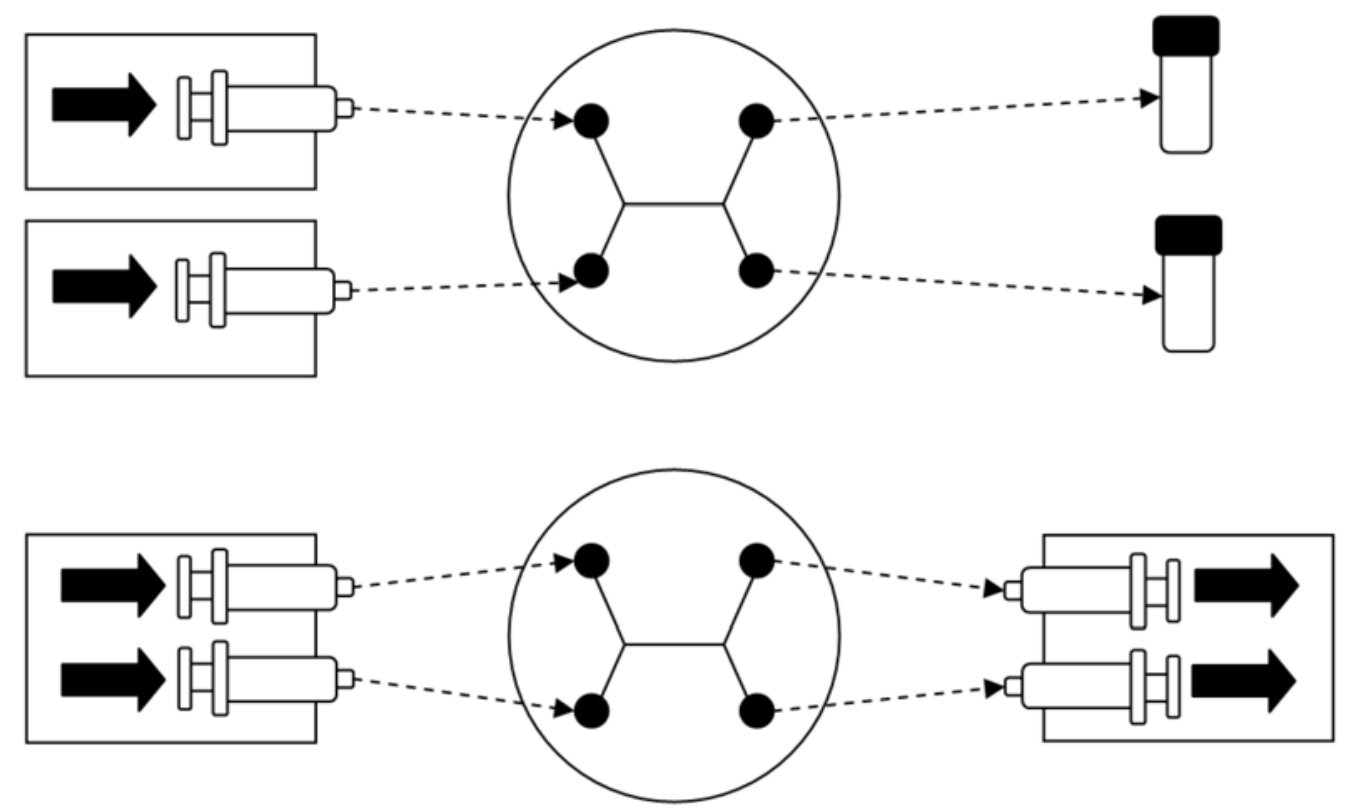

Figure 44: Diagram illustrating the two syringe pump configurations used in the project to establish a laminar flow in a microfluidic device. In the first configuration (top), the input syringes are independently driven, while solution exiting the device is collected in vials. In the second configuration (bottom), the input syringes are driven on the same pump, with the second used to withdraw solution from the outlets to syringes. The second configuration was favoured due to its ability to overcome blockages by bubbles.

\subsubsection{Pressure difference and hydraulic resistance}

When using pressure-driven flow, it is important to consider the magnitude of the pressure difference $\Delta p$ that is required. If this pressure is too great, severe damage may be inflicted on the device. For steady-state flow of an incompressible fluid in a straight channel, this is given by the Hagen-Poiseuille law [4] 


$$
\Delta p=R_{\text {hyd }} Q
$$

where $R_{h y d}$ is the hydraulic resistance of the channel and $Q$ is the volumetric flow rate. The hydraulic resistance is dependent on both the size and shape of the channel crosssection, as well as its length. Regardless of channel shape, the resistance increases with the inverse cube of cross-sectional area, meaning a much greater pressure difference is required to maintain a flow in a microchannel compared to a conventional pipe. The channels in devices produced for this project were approximately rectangular in shape, for which the hydraulic resistance is given by [4]

$$
R_{h y d}=\left(\frac{12 \eta L}{1-0.63(h / w)}\right) \frac{1}{h^{3} w}(h<w),
$$

where the channel has height $h$, width $w$ and length $L$, as illustrated in Figure 45. The fluid has a dynamic viscosity given by $\eta$, equal to $1 \mathrm{mPa} \cdot \mathrm{s}$ for water. In this project channel dimensions of $h=150 \mu \mathrm{m}, w=300 \mu \mathrm{m}$ and $L=25 \mathrm{~mm}$ were typical. This channel produces a hydraulic resistance of $4.3 \times 10^{11} \mathrm{~Pa} \cdot \mathrm{s} \cdot \mathrm{m}^{-3}$. Equation (33) then implies a pressure difference of $36 \mathrm{~Pa}$ is required to achieve a flow rate of $5 \mu \mathrm{L} / \mathrm{min}$, which was the slowest flow rate used in this project. The largest flow rate used was $500 \mu \mathrm{L} / \mathrm{min}$, which requires a pressure difference of $3.6 \mathrm{kPa}$. As will be discussed in Section 4.3.3, the final generation of microfluidic devices fabricated during this project were capable of withstanding much larger pressures than this. Equation (34) does not consider the added force needed to wet the hydrophobic surface of the PDMS with a hydrophilic liquid (such as water). It also does not provide the magnitude of pressure spikes, which should be produced when the flow is abruptly stopped and started. Finally, if more viscous solutions are flowed through the device, greater pressures will result.

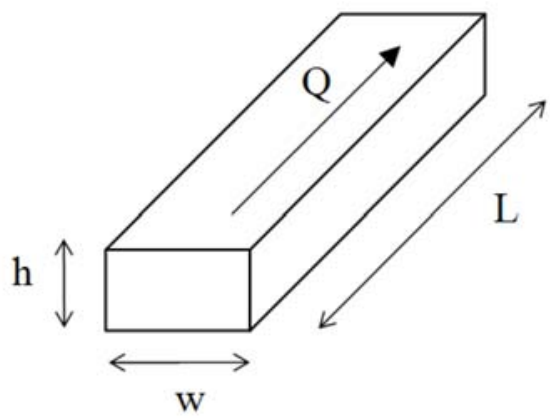

Figure 45: Definition of dimensional parameters for Equation (34) for a rectangular channel cross-section. The direction of fluid flow is indicated by $Q$. 


\subsection{Particle and solution characterisation}

In this section, important techniques used to characterise the properties of particles and solutions are summarised. Vibrating Sample Magnetometry (VSM) and the Superconducting Quantum Interference Device (SQUID) were used to measure the magnetisation behaviour of magnetic microbeads and nanoparticles. Operating principles and sample preparation for these methods are therefore first discussed. Ultraviolet-Visible (UV-Vis) spectroscopy was used to measure the concentrations of magnetic bead suspensions and dye solutions, and is therefore also outlined.

\subsubsection{Vibrating Sample Magnetometry (VSM)}

The Vibrating Sample Magnetometer (VSM) was introduced by Foner [185] in 1959, and quickly became a popular tool for the measurement of magnetisation curves, as it is relatively simple and inexpensive to use. The general layout of a VSM is shown in Figure 46. The sample is magnetised by placing it in the spatially-uniform field of a large electromagnet. The magnitude of this field is monitored using a Hall probe, and the electromagnet current may be adjusted to change the field. This allows the magnetisation curve of the material to be constructed. The sample magnetisation at a given value of the applied field is measured by exploiting Faraday's Law of Induction, which states that a changing magnetic field will produce an electric field. This changing magnetic field is generated by vibrating the sample at a known amplitude and frequency, usually using a piezoelectric material. The stray field of the sample will then change as a function of time, producing a current in the detection coils at the frequency of the vibration. The periodicity of the induced current allows lock-in amplification techniques to be used, greatly increasing the signal-to-noise ratio of the system. As a result, magnetic moments as small as $10^{-7} \mathrm{~J} / \mathrm{T}$ can be detected using an optimised system [186]. 


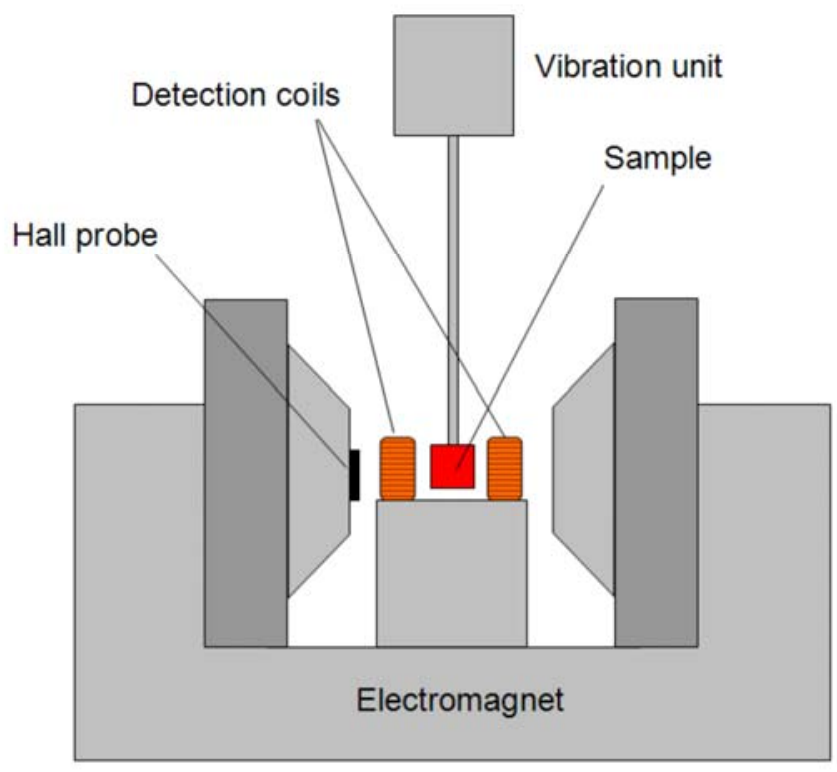

Figure 46: Schematic diagram of a Vibrating Sample Magnetometer (VSM).

In this project, magnetisation curves were measured using a VSM (Lakeshore Cryotronics [186]) located at Industrial Research Ltd, which has a sensitivity of $10^{-6} \mathrm{~J} / \mathrm{T}$. The electromagnet is able to supply fields of $\pm 1 \mathrm{~T}$. All samples were measured in powder form. A known mass was transferred into the longer half of a size 2 gelatin capsule (diameter $6.4 \mathrm{~mm}$ ). The capsule was then packed with Teflon tape to immobilise the particles, as movement inside the capsule could affect measurements. The capsule was then pressed tightly onto the end of a PMMA stub, which itself was screwed onto the end of the VSM sample rod. It should be noted that all materials used in the mounting process are nonmagnetic, meaning they make only a weak diamagnetic contribution to the measured magnetic moment. This contribution was found to be at most on the order of $10^{-6} \mathrm{~J} / \mathrm{T}$, which coincides with the inherent noise level of the instrument.

\subsubsection{Superconducting Quantum Interference Device (SQUID)}

The Superconducting Quantum Interference Device (SQUID) is one of the most sensitive magnetometers available, with the potential to detect fields as low as $10^{-15}$ tesla [187]. The instrument makes use of low-temperature superconducting coils, and therefore requires the use of liquid helium to operate, making it much more costly than the VSM. Nonetheless the improved sensitivity, and ability to measure the temperature dependence of the sample magnetisation, makes it a preferred option in some cases. 
In this project a Magnetic Property Measurement System (Quantum Design Inc [188]) at Industrial Research Ltd was used to perform SQUID measurements. This system has a sensitivity of $10^{-9} \mathrm{~J} / \mathrm{T}$. The detection system of the SQUID is shown in Figure 47 . The sample is moved vertically inside a small chamber, which is surrounded by a set of superconducting pickup coils. Both are encapsulated in a liquid helium jacket, which keeps the coils below their superconducting transition temperature, and also enables optional cooling of the sample. A superconducting wire bundle forms a larger coil around the jacket, which is used to provide the applied field. Using a superconducting coil allows applied fields of up to $7 \mathrm{~T}$ to be used.
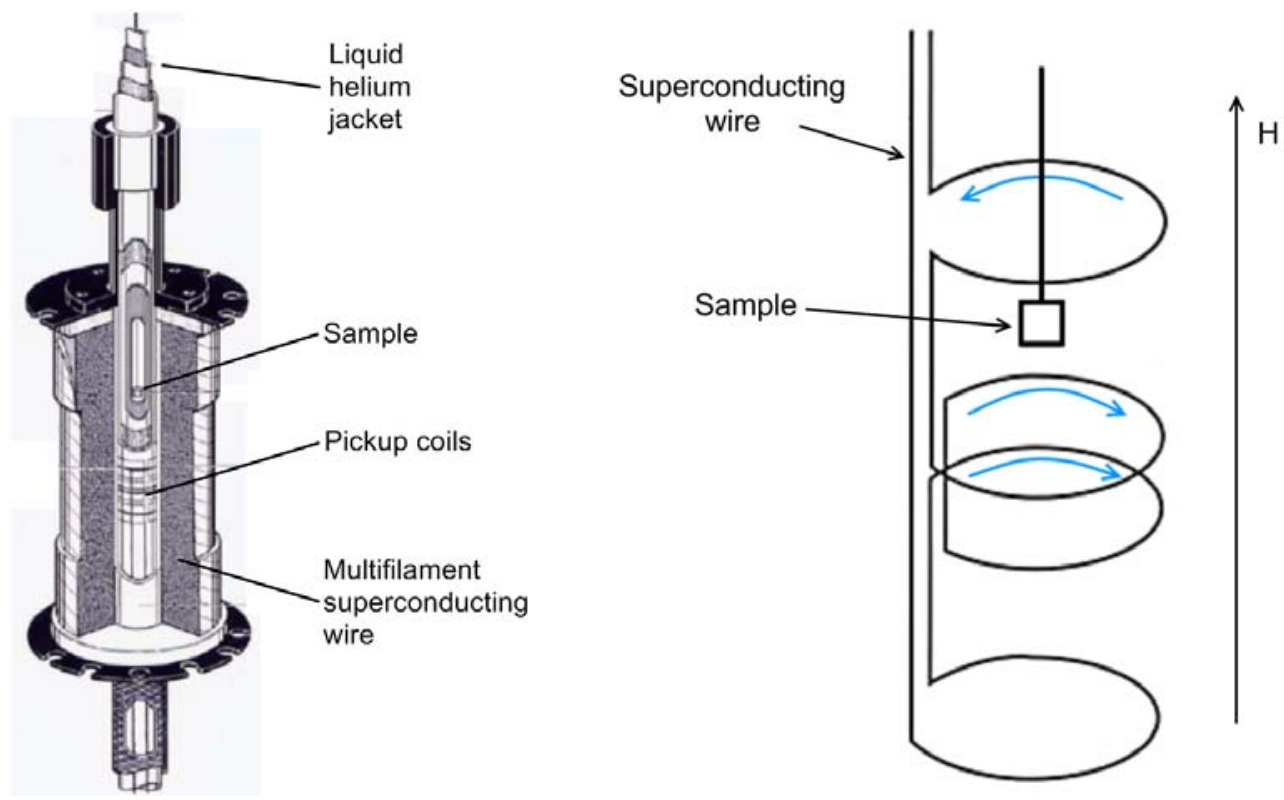

Figure 47: (Left) Cross-sectional view of a SQUID sample chamber, showing superconducting wire for applying a magnetic field, and superconducting pickup coils for detection of the sample magnetisation. (Right) The change in magnetic flux as the sample moves through the pickup coils induces a change in current, indicated by the blue arrows. Image adapted from [189].

The coil arrangement is also shown in Figure 47. Each measurement of the magnetisation involves moving the sample vertically through the coils. As the sample is moved, the resulting change in magnetic flux induces currents in the coils. Because the coils have no resistance, these currents will not dissipate after the sample stops moving. It is only strictly necessary to use a single coil, but the counter-turns in the arrangement shown help to cancel out unwanted variations in the applied field. 
Using the term SQUID to describe the magnetometer is somewhat misleading, as the SQUID itself is merely one part of the detection electronics. The SQUID is connected to the superconducting coil loop, and acts as an extremely sensitive current-to-voltage converter. For the coil arrangement shown, the voltage output as the sample moves through the coils ideally follows the form in Figure 48. Computer software is typically then used to calculate the magnetisation from the voltage output. The fit typically assumes a magnetic dipole, so it is important that the sample is small compared to the coils.

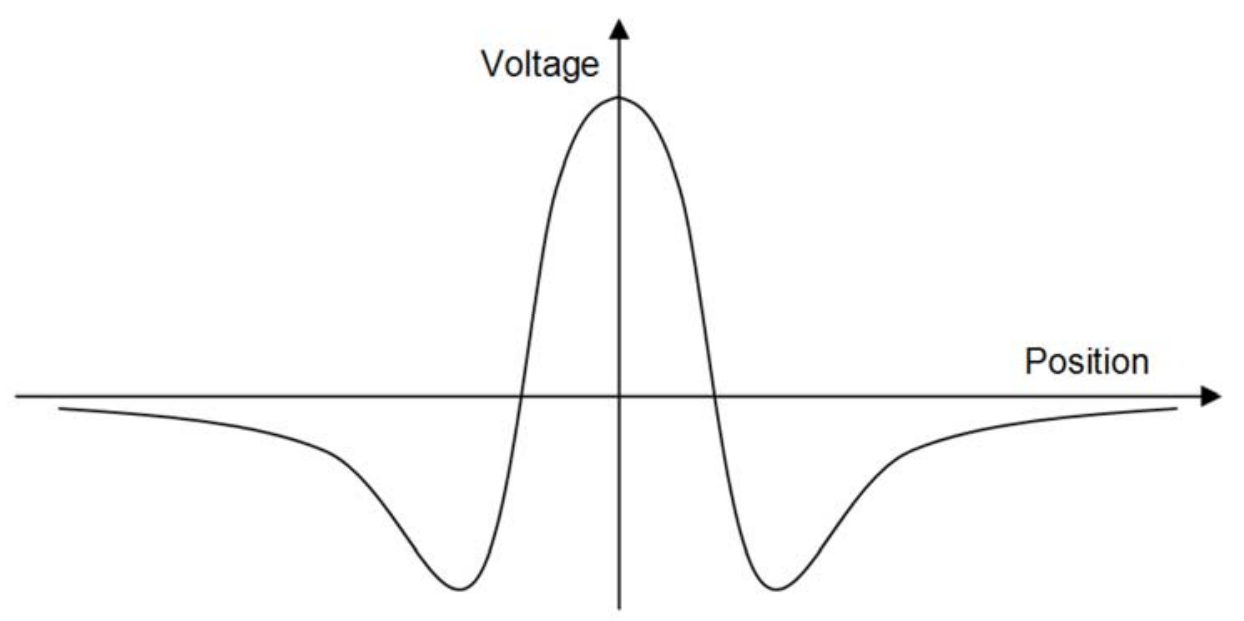

Figure 48: SQUID output voltage as a function of sample position, relative to the midpoint of the coils.

All samples measured using the SQUID were prepared in powder form, as was the case for the VSM measurements. However, the lower noise of the SQUID makes minimising the diamagnetic background from the sample holder more important than it was for VSM. A known mass of sample was transferred into the cap of a size 4 capsule (diameter $5.35 \mathrm{~mm}$ ). The body of the capsule was then pushed into the cap to immobilise the powder, and a hot wire used to weld the capsule shut. The sealed capsule was then inserted into a plastic drinking straw, along with the two halves of a second straw. The outer straw was then attached directly to the sample rod. The final configuration is shown in Figure 49. When this configuration is used the magnetic powder is contained to a small volume, meaning the magnetic dipole assumption is justified. Additionally, the sample holder arrangement is essentially a pair of plastic straws on the same axis. The straws are longer than the pickup coil arrangement, meaning their net contribution to the magnetic moment will cancel out. 


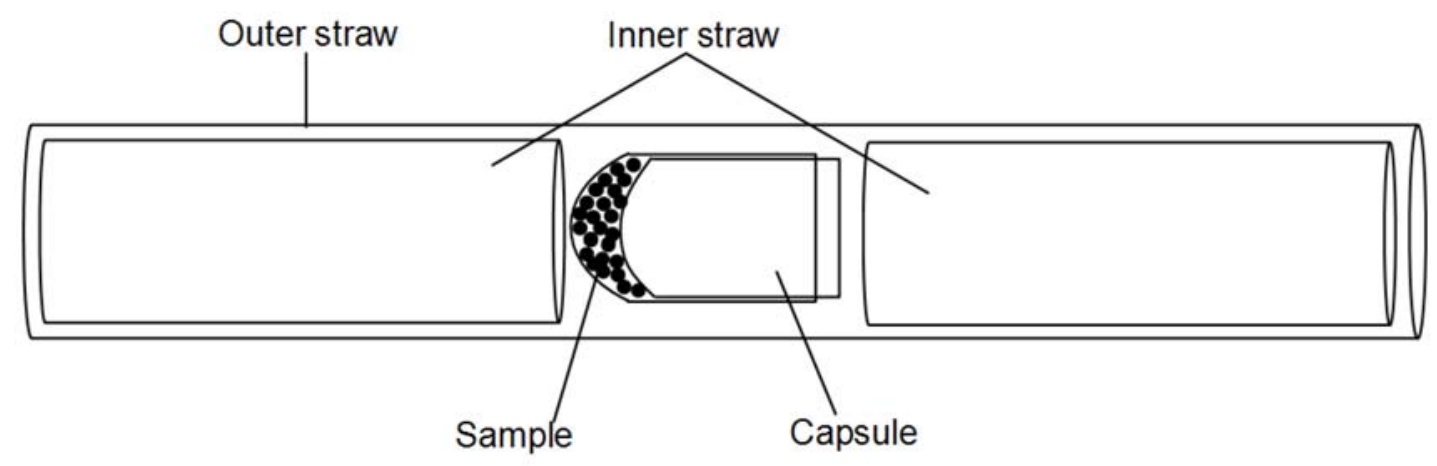

Figure 49: Sample holder configuration used for SQUID measurements.

\subsubsection{UV-Visible spectrometry}

UV-Visible spectrometry was used extensively to determine the concentration of bead dispersions and dye solutions. Light is passed through the sample to a detector. The beam is passed through a diffraction grating either before or after entering the sample, allowing transmission to be measured as a function of wavelength. It is often useful to instead consider the absorbance of the solution, given by

$$
A=-\log _{10}\left(I / I_{0}\right),
$$

where $I$ and $I_{0}$ are the intensities of the transmitted and incident light respectively, as represented in Figure 50.

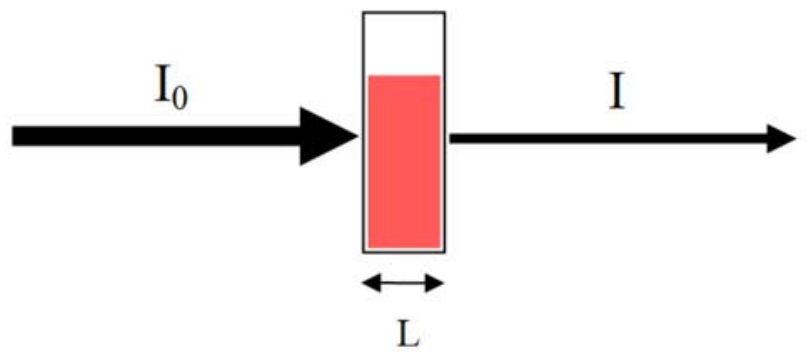

Figure 50: Attenuation of light by an absorbing solution. Incident light of intensity $I_{0}$ is partially absorbed by the solution, and the beam exits with a reduced intensity $I$. The attenuation depends on the sample, as well as the path length $L$.

$\mathrm{UV}-\mathrm{V}$ is is often used to determine the composition of solutions, particularly those containing transition metal complexes. Alternatively, the absorbance of a known solution at a single wavelength can be related to the concentration using the empirical Beer-Lambert law

$$
A=\varepsilon \cdot c \cdot L,
$$


where $\varepsilon$ is the molar absorptivity of the material, $c$ is the concentration and $L$ is the path length. This linear relationship between concentration and absorbance is followed closely in practice for sufficiently dilute solutions. Deviations from linearity occur at higher concentrations, making it necessary to dilute the sample before measurement. In this project, measurements were mostly performed on dispersions of microparticles. At high concentrations, an increasing number of particles will cast a shadow on others, leading to an underestimate of the bead concentration. Additionally, as the absorbance becomes large, the effect of stray light becomes increasingly significant.

Concentration measurements were performed using a Cary 100 UV-Visible spectrophotometer. As can be seen in Figure 51, this is a dual beam spectrometer. One beam is passed through a cuvette containing the sample, with the other being directed through a reference sample. This allows any absorption or scattering by the cuvette or solvent to be subtracted from the absorbance measurement. All measurements were performed using $3.5 \mathrm{~mL}$ optical glass cuvettes (Carl-Roth [190]), which have a path length of $1 \mathrm{~mm}$. These transmit light more effectively than disposable plastic cuvettes, increasing the sensitivity of measurements. However, the glass will absorb light at wavelengths below $320 \mathrm{~nm}$, making it important to conduct measurements outside this range.
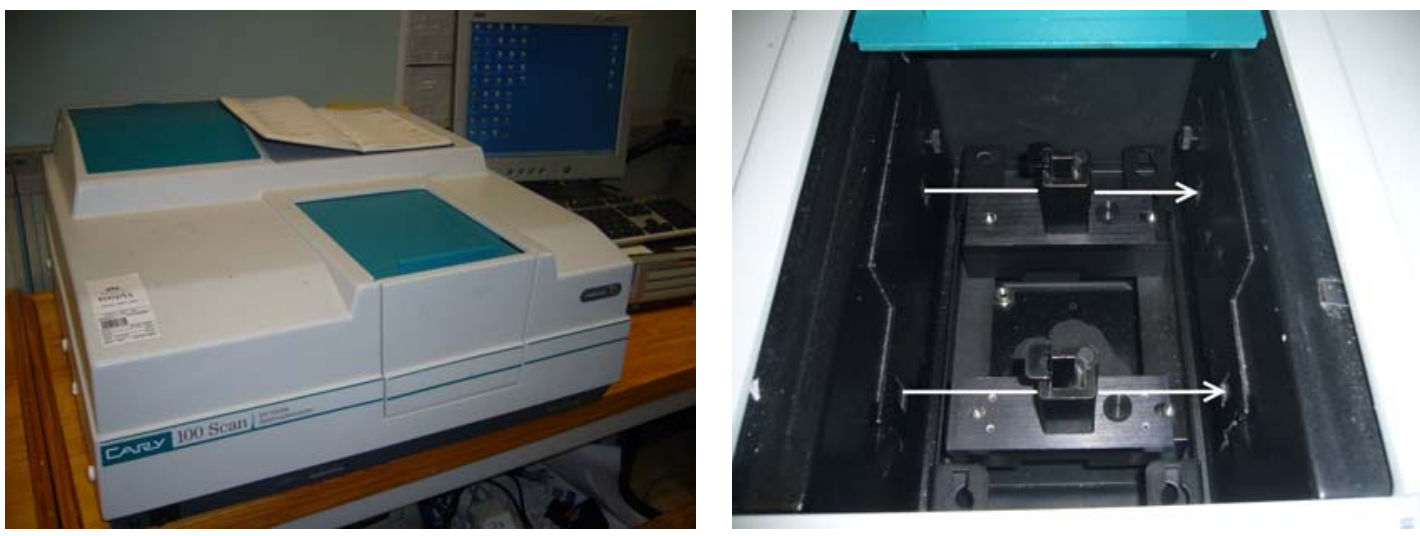

Figure 51: (Left) Cary 100 UV-Visible spectrometer used to measure the concentration of dye solutions and dispersions of microbeads. (Right) Top view of sample chamber, showing cuvette holders for reference and target samples. Beam paths are indicated by white arrows.

In order to determine the range of concentrations for which the Beer-Lambert law could be applied, calibration curves were constructed for all solutions to be measured. The calibration for the dye solution used in Section 4.3.2 is reproduced in Figure 52. The dye 
possessed a broad absorption peak centred at $507 \mathrm{~nm}$, and hence measurements were performed at this wavelength. A strong linear relationship between absorbance and concentration is observed provided the absorbance is less than 2. It was found that the absorbance would tend towards a constant value if the concentration was further increased. In light of this behaviour, investigation of the flow behaviour in a microchannel conducted in Section 3.5.2 used dye at a maximum concentration of 1.25 $\mu \mathrm{L} / \mathrm{mL}$.

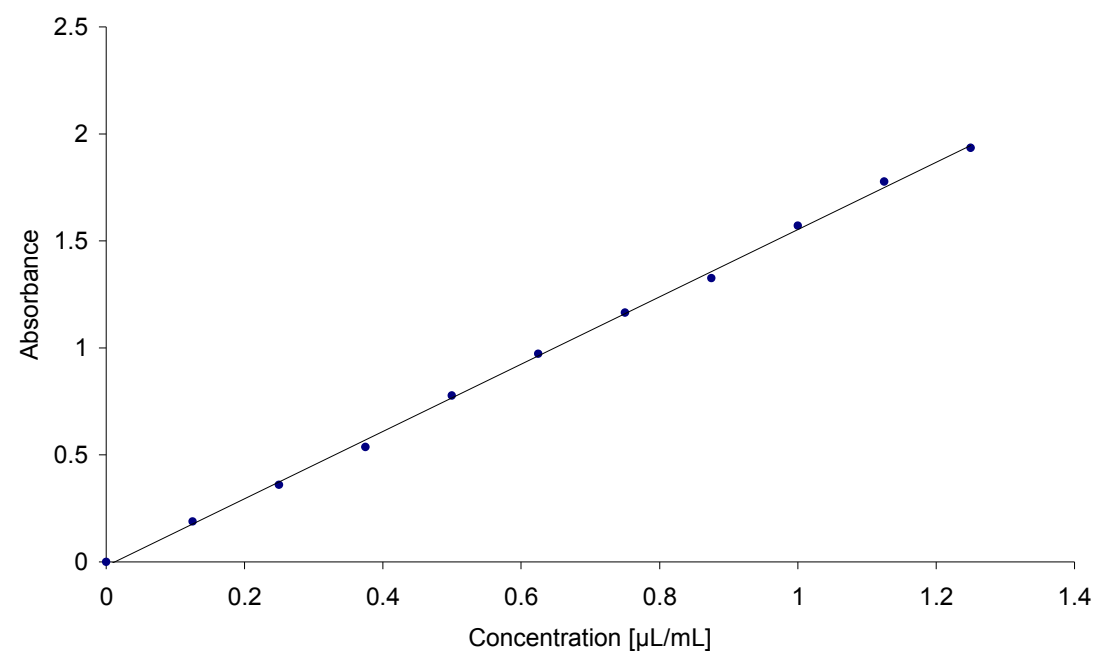

Figure 52: Calibration curve for food dye solution, showing a linear relationship between absorbance and concentration. At higher concentrations saturation in the response begins to occur.

Similar curves obtained for Dynabead MyOne and Polysciences Polybeads microbeads are shown in Figure 53. Measurements were performed at wavelengths giving maximum absorption (605 $\mathrm{nm}$ for the Dynabeads and $320 \mathrm{~nm}$ for the Polybeads). The BeerLambert law is once again followed for values of the absorbance below 2. Saturation of the absorbance would occur at higher concentrations, as was seen previously with the dye solution. It should be noted that higher concentrations may still be quantified by first diluting the solution. Provided the dilution factor is known, the original concentration can then be easily determined.

The calibration results presented in this section make it clear that UV-Vis spectrometry is a useful tool for determining the concentration of the solutions. This was particularly significant for measurements of the magnetic separation efficiency for Section 4.4. Alternative methods include magnetometry or mass measurements, which both require 
larger amounts of material to yield accurate results. Sample preparation is also more time consuming, meaning fewer experiments could have been conducted.
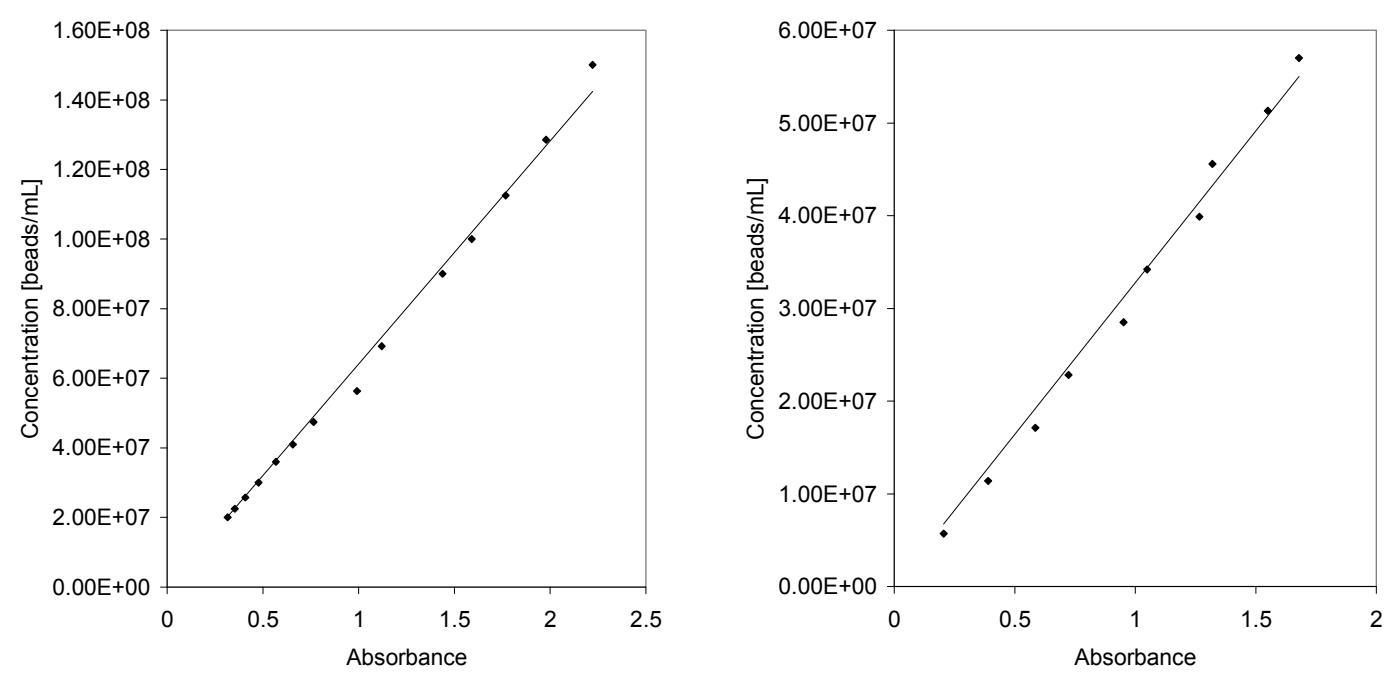

Figure 53: Calibration curves for Dynabeads MyOne (left) and Polysciences Polybeads (right), showing a linear relationship between absorbance and concentration. At higher concentrations saturation in the response begins to occur.

\subsection{Flow characterisation}

In Section 2.3.2 it was predicted that the Reynolds number is very low in a microfluidic channel, meaning the fluid flow within the channels should be laminar. The design of the magnetic separation device for this project aimed to leverage this effect. In order to confirm laminar flow was actually present in the device geometry used, experiments were conducted to investigate the flow of both dye and microparticle solutions in the device.

In this section, the protocol for these experiments will be outlined. Details of the sample preparation methods are discussed, followed by a description of the flow experiments themselves. Finally, measurements of mixing in the microchannels using microscopy and spectroscopy are described.

\subsubsection{Sample preparation}

Tests to directly image fluid flow in the microchannels were performed using a concentrated dye solution. The dye used was a commercially available red food dye (Queen Fine Foods [191]). The solution was prepared by diluting several drops of the 
dye in $10 \mathrm{~mL}$ of distilled water. It was not necessary to know the exact concentration of the dye in this case, as no quantitative analysis was to be performed. It was however required that the dye solution be made up to a high concentration, in order for the very small volume present in the microchannel to remain visible.

Subsequent tests, in which fractions were collected for UV-Vis spectroscopy, required a known dye concentration. The concentration needed to be sufficiently small to avoid nonlinearity in the spectrometer response. Solutions were prepared in distilled water at a concentration of $1.25 \mu \mathrm{L} / \mathrm{mL}$ using a micropipette. The pipette was only accurate down to volumes of $50 \mu \mathrm{L}$, so the solution was diluted down to the target concentration in steps.

To ensure similar flow behaviour is displayed by a dispersion of particles, quantitative experiments were also performed using a suspension of $1 \mu \mathrm{m}$ polystyrene latex beads (Polysciences Inc [192]). The beads are supplied in aqueous suspension, and are stable when diluted in distilled water. Like the food dye solution, this suspension must be sufficiently dilute to avoid nonlinearity in the spectrometer response. A $3 \mu \mathrm{L} / \mathrm{mL}$ suspension of the beads was therefore prepared, using the same process described for the food dye. The concentration is equivalent to a particle concentration of $1.4 \times 10^{8}$ beads/mL. It was important to ensure that no sedimentation of the beads occurred during the experiments, as this could skew the results. However, it was found that the beads remained dispersed for many hours, well beyond the length of an experimental run. If the suspension was used for an experiment several days after preparation, the beads could be easily re-dispersed by vigorously swirling the solution bottle.

\subsubsection{Experimental protocol}

In all flow characterisation experiments, the target solution was flowed through one inlet at a constant flow rate, with distilled water flowed through the second inlet at the same flow rate. In cases where images of the flow were taken, solution exiting the device was discarded. If fractions were to be collected, the device outlets were connected to sample vials, as pictured in Figure 54. The configuration used is described in more detail in Section 3.3.1, where attention was drawn to the issue of bubble formation in the 
channels. This can in some cases obstruct a single outlet, and divert solution into the adjacent outlet. In hindsight it would have been preferable to apply negative pressure at the outlets to prevent this occurring. However, there was found to be no difference in volume between the two fractions collected at each outlet in these cases, suggesting no such obstructions occurred. It is likely that the high flow rates used prevented bubbles from becoming trapped in the channel.

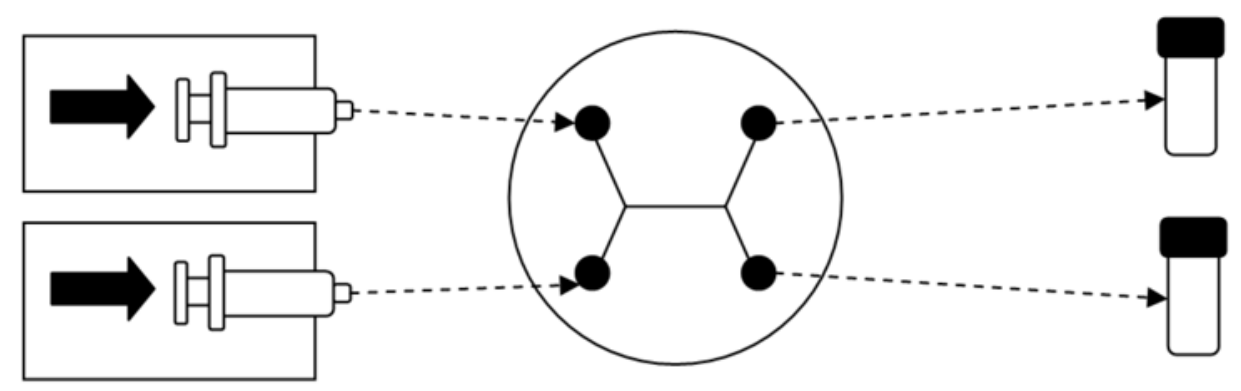

Figure 54: Syringe configuration used for laminar flow characterisation experiments. Fractions were collected in sample vials for spectroscopy. Image reproduced from Figure 44.

Experiments to image the flow of dye through the channels were performed at 250 $\mu \mathrm{L} / \mathrm{min}$ and $500 \mu \mathrm{L} / \mathrm{min}$, using $1 \mathrm{~mL}$ syringes. Runs to obtain fractions for spectroscopy required a larger solution volume, meaning $3 \mathrm{~mL}$ (dye experiments) or $5 \mathrm{~mL}$ (bead experiments) syringes were used in these trials. The increase in volume also helped to reduce the significance of dead volumes in the microfluidic device, which would otherwise lead to a reduction in the measured solution concentration.

\subsubsection{Observation methods}

Images were captured using an optical transmission microscope, with a Nikon D200 digital camera attachment. The microfluidic device was placed on the microscope stage throughout the run. The channel was observed and imaged using a $4 \mathrm{x}$ objective. Higher magnifications were available but unused, as the amount of light transmitted was inadequate. Before experiments were started, adhesive tape was used to clean the outer surfaces of the PDMS.

During each experiment pictures were taken at the midpoint of the central channel, as well as at both the inlet and outlet junctions. It was necessary to physically move the device on the microscope stage (by hand) to observe different regions of the channel, 
which temporarily disrupted the flow balance. The balance between the dye and buffer flows would however recover within a few seconds. Whenever the device was moved, time was allowed for the flow to stabilise before taking an image.

During experiments where fractions were to be collected for analysis, the microfluidic device was not placed on the microscope stage. The device was instead rested on a benchtop next to the pumps. This provided a more stable surface, helping to minimise disruptions to the flow.

The concentration of collected fractions was measured using UV-Vis spectroscopy. Details of the method were provided in Section 3.4.3. The dye used was found to have a broad peak in absorbance in the visible region, centred at $\lambda=507 \mathrm{~nm}$. The absorbance of samples at this wavelength was measured, and converted to an equivalent concentration using the calibration curve presented in Figure 52. Fractions from experiments using Polybead microspheres were characterised in a similar fashion. Absorbance was measured at a wavelength of $320 \mathrm{~nm}$, and related to concentration using Figure 53.

\subsection{Magnetic separation}

In this section, experiments to determine the magnetic separation efficiency of the microfluidic devices are introduced. Several different solutions of magnetic and nonmagnetic particles were prepared for various experiments, and these are summarised first. This leads into a description of the basic protocol for separation experiments, including the collection of fractions. The section closes with a discussion of the techniques used to qualitatively and quantitatively observe the magnetic separation efficiency.

\subsubsection{Sample preparation}

Most of the magnetic separation experiments were performed using Dynabeads MyOne $1 \mu \mathrm{m}$ magnetic beads (Invitrogen [193]). These were purchased in $2 \mathrm{~mL}$ quantities, which were supplied in aqueous suspension. The nominal particle concentration is $(9.5 \pm 2.5) \times 10^{9}$ beads $/ \mathrm{mL}$, or $10 \mathrm{mg} / \mathrm{mL}$ [193]. The vial was shaken for a couple of minutes before extracting the solution, to ensure the beads were well dispersed. The beads are heavier than water, and would settle to the bottom of the vial if left standing 
for more than a few hours. The beads were transferred from the vial into a $1.5 \mathrm{~mL}$ microcentrifuge tube using a micropipette. The solution was then diluted in distilled water to obtain the desired bead concentration.

In most quantitative experiments, a mixture of 1 part bead solution to 11 parts water was used. This should give a bead concentration of $8 \times 10^{8}$ beads $/ \mathrm{mL}$, although it was found in practice that the resulting concentration was closer to $6.5 \times 10^{8}$ beads $/ \mathrm{mL}$. This concentration was large enough to be measured using UV-Vis spectroscopy without further enrichment. In experiments intended for imaging the separation process, a higher concentration was needed to create sufficient contrast. Therefore, a mixture of 3 parts bead solution to 1 part water was instead used. This should give a concentration of $7 \times 10^{9}$ beads $/ \mathrm{mL}$, although measurements again suggested a slightly lower concentration of $6 \times 10^{9}$ beads $/ \mathrm{mL}$ was obtained. Using such high concentrations meant it was necessary to recycle the solution for multiple runs to conserve material. Gradual depletion of the recycled solution was offset by adding a small amount of fresh solution, to roughly maintain the original solution colour. This reuse of particles led to significant variation in the actual concentration, making direct comparison between separate experiments problematic.

Experiments to separate particles based on their magnetic moment (see Section 4.4.3) required the use of nonmagnetic particles. The Polybead polystyrene microspheres used in Section 3.5 were also used for this purpose. Because these beads are of a similar size to the Dynabeads, fluid drag should be comparable between the types. Polystyrene has a density of $1.05 \mathrm{~g} / \mathrm{cm}^{3}$, only slighter denser than water, meaning sedimentation occurs much slower than for the heavier magnetic beads. Nonetheless, the solution was shaken before use to ensure complete dispersion. Solutions containing 1 part Polybeads : 1 part Dynabeads : 10 parts distilled water were used for the relevant experiments. This corresponds to a Polybeads concentration of $3.8 \times 10^{9}$ beads $/ \mathrm{mL}$, and a Dynabeads concentration of $8 \times 10^{8}$ beads $/ \mathrm{mL}$. Spectroscopic measurements suggested actual concentrations of $3.48 \times 10^{9}$ Polybeads $/ \mathrm{mL}$ and $6.56 \times 10^{8}$ Dynabeads $/ \mathrm{mL}$.

Experiments to separate magnetic beads based on their size were performed using two types of magnetic beads from Spherotech, PM-20-10 and PM-50-10 [194]. These consist 
of iron oxide nanoparticles embedded in polystyrene latex, and have particle diameters of $2 \mu \mathrm{m}$ and $5 \mu \mathrm{m}$ respectively. The beads were supplied in aqueous suspension in 0.5 $\mathrm{mL}$ quantities. The concentration was calculated to be approximately $4 \times 10^{9}$ beads $/ \mathrm{mL}$ for the $2 \mu \mathrm{m}$ beads, and $2 \times 10^{8}$ beads $/ \mathrm{mL}$ for the $5 \mu \mathrm{m}$ beads. The preparation protocol was similar to that used for the Dynabeads, except that the smaller sample holders made it more difficult to agitate the solution. It was found that flicking the sample holder helped to overcome surface tension between the solution and container walls, allowing the particles to be redispersed. Size selection experiments were conducted using a mixture of 1 part PM-20-10 : 1 part PM-50-10 : 20 parts distilled water. This is equivalent to a concentration of $2 \times 10^{8}$ beads $/ \mathrm{mL}$ for the $2 \mu \mathrm{m}$ beads, and $1 \times 10^{7}$ beads/mL for the $5 \mu \mathrm{m}$ beads.

Suspensions of particles were often prepared several hours before use, and in some cases were used more than once. Care therefore needed to be taken to ensure that the particles were redispersed immediately before use. This was best achieved by shaking the microcentrifuge tube holding the sample for a few minutes. A drawback of this method was that air bubbles could form in the solution, which may lead to blockages in the microfluidic channels. Sonication was investigated as an alternative approach to mixing, but the dispersion was generally poorer than that obtained by hand.

Iron and iron carbide nanoparticles were prepared from a dry powder. Samples used were supplied by Ferguson [181], as dispersions prepared by the author sedimented too rapidly. The samples were dispersed in ethanol at a concentration of about $6 \times 10^{13}$ particles/mL. Shaking the sample holder appeared to disperse the particles well, but to maximise the stability of the dispersion the sample was sonicated for several minutes directly before separation.

\subsubsection{Experimental protocol}

The majority of the magnetic separation experiments were performed using the magnetmicrometer system, although a small number were also performed using the Metglas system. No formal experiments were conducted using the embedded magnet system, as 
the inability to remove the magnetic field and flush trapped particles made it a less promising option for testing.

The flow configuration used for all separation experiments is shown in Figure 55. The solution containing the particles was flowed into the channel on the side opposite the magnet. A buffer solution was simultaneously flowed into the channel on the same side as the magnet, to collect particles diverted by the magnetic field. As discussed in Section 3.3.1, using the second syringe pump to apply negative pressure at the outlets helped to prevent bubbles becoming trapped in the channel. This prevented fluid being diverted to the incorrect outlet, but meant the buffer and solution must be flowed at the same rate. Fortunately, in almost all cases there was no need to run the two fluids at different rates.

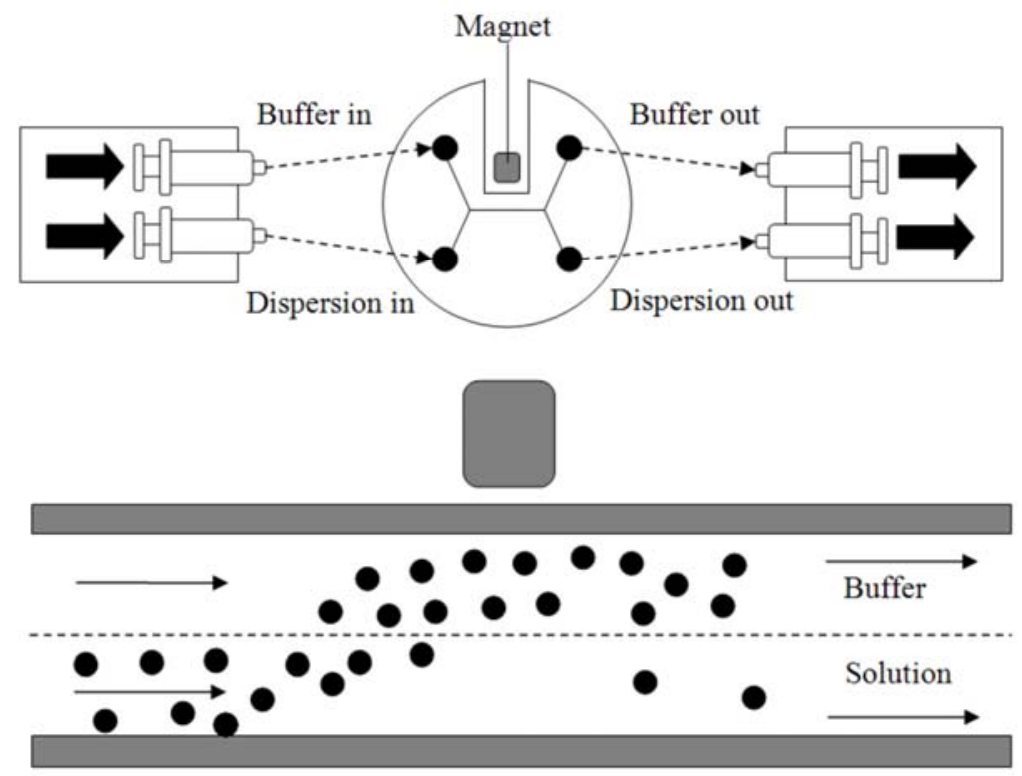

Figure 55: Syringe pump configuration used in all magnetic separation experiments

Before each experiment was performed, the microfluidic device channels and tubing were first flushed. A few millilitres of distilled water was forced through the channel at a very high flow rate $(>2000 \mu \mathrm{L} / \mathrm{min})$. The same process was repeated with air in the syringes, to eject as much of the water as possible.

Use of the micrometer setup allowed the position of the magnet (and therefore the strength of the field gradient) to be modified. In practice this was only used in experiments conducted to investigate the effect of changing the magnetic field gradient. 
In all other cases the magnet was positioned as close as possible, and the flow rate then chosen to optimise the separation efficiency.

\subsubsection{Observation techniques}

In experiments where the sole aim was to image the behaviour of magnetic particles in the applied field, the flow rate was often changed mid-run. The flow rate was adjusted until the majority of particles were seen to migrate across the laminar flow boundary, without getting trapped against the channel sidewall. In some cases the effective field gradient was also adjusted mid-run, by altering the magnet position. Images were taken using a transmission optical microscope with a Nikon D200 camera attachment, as described in Section 3.5.3. All images were taken at $4 \mathrm{x}$ magnification, although focussing was first performed at $16 x$ to improve focus. It was necessary to physically move the microfluidic device in order to enable photographs to be taken at multiple points. Because the body of the device is made from compressible PDMS, direct handling can cause pressure changes in the channel. However, the magnet-micrometer system is supported on a rigid Perspex stage, which allows it to be moved without directly handling the PDMS.

In experiments conducted for quantitative measurements of the separation efficiency, the flow rate was held at a constant value throughout the run. The particle dispersion used for these experiments was too dilute to be directly observed using the microscope, so the microfluidic device was instead rested on the benchtop. Once the run was complete, fractions were collected for spectroscopic analysis, from a number of positions marked in Figure 56. The fractions were given the following labels:

- Original $(O)$ refers to the solution prepared for the experiment. Leftover solution was often used as a standard.

- Inlet (I) refers to particles that sedimented in the solution tubing before entering the microfluidic device.

- Separated_1 (S1) refers to particles that were drawn into the buffer stream and then withdrawn into the outlet tubing or syringe.

- Separated_2 (S2) refers to particles that were drawn into the buffer stream but then settled in the base of the luer connector rather than exiting the device. 
- Unseparated_1(U1) refers to particles that remained in the solution stream and were then withdrawn into the outlet tubing or syringe.

- Unseparated_2 (U2) refers to particles that remained in the solution stream and settled in the base of the luer connector.

Sedimented particles were collected by flushing each luer adaptor with distilled water, and then withdrawing the solution. The volume of each fraction was inevitably different, so direct comparisons of concentration were meaningless. Instead, the particles were precipitated using a magnet, and the solution decanted. The fractions could then be redispersed in equal volumes of distilled water for analysis.

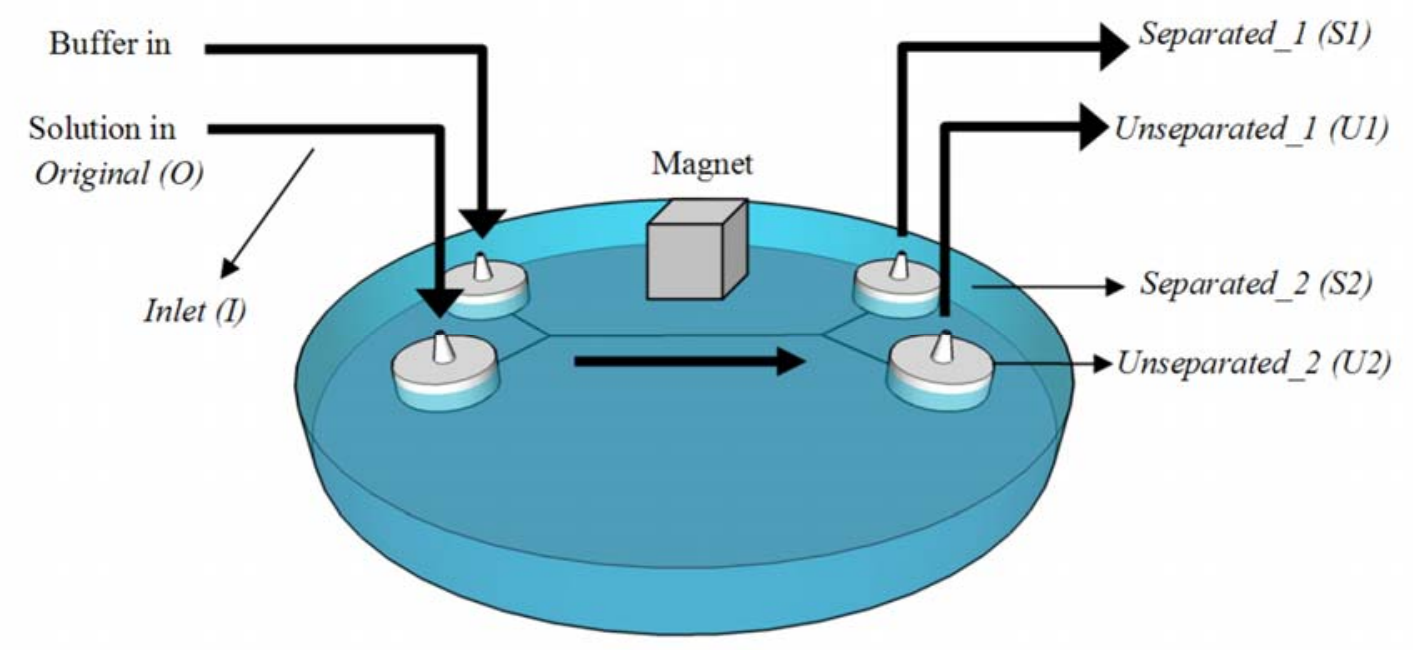

Figure 56: Schematic showing positions from which fractions were collected for spectroscopic analysis of particle concentration. Bold arrows indicate the direction of fluid flow. Fraction names are italicised.

A number of experiments involved the separation of magnetic Dynabeads from nonmagnetic Polybeads. Here it is not sufficient to measure the concentration of each fraction directly, as spectrometry cannot distinguish between the different particle types. To overcome this issue, each fraction was subsequently split into a magnetic and nonmagnetic component. This allowed the concentration of each particle type to be measured independently. A permanent magnet was used to force precipitation of the Dynabeads. The Polybeads would remain dispersed in the solution, allowing them to be transferred to a new holder using a pipette. 
Some experiments were conducted to select one size of magnetic bead over another. The method discussed above for isolating the particle types is not applicable, as both types will respond to the magnet. Relative proportions of the two types were therefore determined for each fraction using optical microscopy. This did not allow the absolute particle concentration to be determined, but changes in the relative concentrations may still be examined. For each fraction, the solution was agitated to ensure a uniform dispersion of particles. A few drops of solution were then deposited on a glass microscope slide, and the liquid was allowed to evaporate. Once the slides were prepared, micrographs were taken at 100x magnification using a backlit optical microscope. For each fraction, micrographs were taken at several positions on the slide. The populations of each particle size were manually counted from these images.

Experiments involving the separation of magnetic nanoparticles required yet another measurement approach. As discussed in Section 3.6.1, the particles were dispersible in aqueous solvents. However, the level of dilution required to collect the volumes needed for spectroscopy was likely to cause precipitation, by reducing the relative amount of surfactant present [181]. The dispersion was instead converted back to a powder, by allowing the ethanol to evaporate, and then weighed on a mass balance. It was expected that the DMSA surfactant would contribute to the measured mass, but the low density of the surfactant compared to the magnetic particles should make this a minor contribution. Furthermore, provided that the microfluidic magnetic separation process does not strip surfactant from the surface of the nanoparticles, this should affect comparisons between collected fractions. 


\section{Results}

In this chapter, key results obtained over the course of this project are presented. Measurements of the physical properties of magnetic nanoparticles and microbeads will be presented in Sections 4.1 and 4.2, with an emphasis placed on VSM magnetic measurements. This will be followed by an assessment of the performance of the various microfluidic devices produced, including the three magnetic separation device designs. Finally, results from magnetic separation experiments will be discussed in Section 4.4, focussing on the separation of magnetic microbeads using the magnet-micrometer system.

\subsection{Properties of synthesised nanoparticles}

\subsubsection{Size and morphology}

As discussed in Section 3.2.2, the synthesis method used to make iron nanoparticles yielded two distinct types of particles. Iron oxide particles less than $10 \mathrm{~nm}$ in size constituted the majority of the product, but a smaller number of iron and iron carbide particles, tens of nanometres in size, were also obtained. These particles have a larger magnetic moment, allowing them to be isolated from the bulk using a permanent magnet. The leftover iron oxide is then removed from the solution by centrifugation.

The iron-oxide particles collected by centrifugation were characterised by TEM imaging. The micrographs shown in Figure 57 indicate substantial particle aggregation has occurred. This aggregation is likely to have resulted from an excessive number of purification steps. This reduces the concentration of surfactant molecules in the solution, meaning particles are less likely to be capped by the surfactant, and thus more likely to come into direct contact with one another. Van der Waals forces between the particles will then lead to irreversible aggregation. It is nonetheless possible to resolve individual particles in the image, particularly near the edges of the clusters, where the particles are in a single layer. 

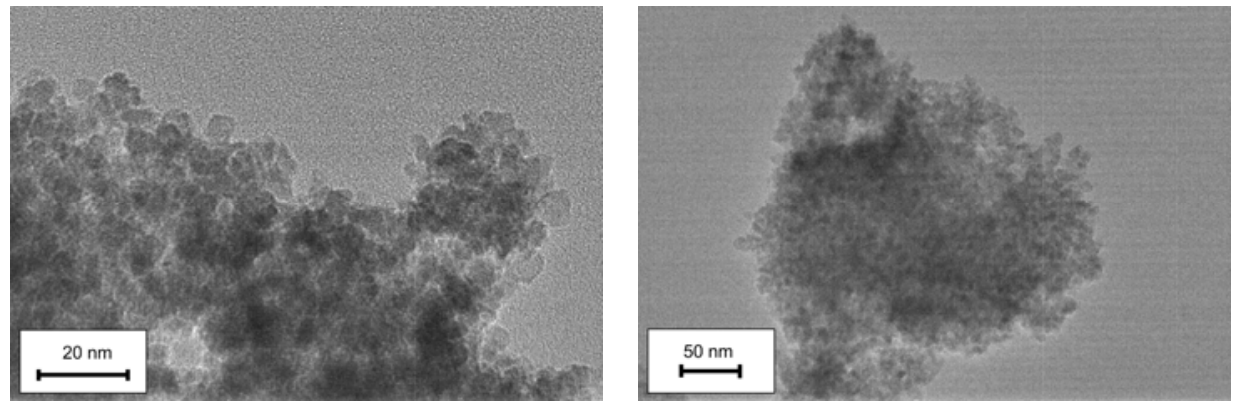

Figure 57: TEM micrographs of iron oxide nanoparticles produced by a thermal decomposition reaction.

Using the left-hand image of Figure 57, a mean particle diameter of (4.8 \pm 1.2$) \mathrm{nm}$ was estimated. From the right-hand image, it can be seen that the particles formed aggregates between 250 and $300 \mathrm{~nm}$ in size. It should be noted that in the original solution this aggregation would not be expected to occur, as an excess of surfactant would be present to keep the particles apart. Electron diffraction experiments performed by Cheong [39] led to the conclusion that the particles were predominantly magnetite $\left(\mathrm{Fe}_{3} \mathrm{O}_{4}\right)$ or maghemite $\left(\mathrm{Fe}_{2} \mathrm{O}_{3}\right)$. Both materials possess an identical spinel structure, meaning that diffraction experiments could not be used to distinguish between them. The bulk powder is generally a dark black colour, rather than brown, suggesting magnetite is the more likely phase. Magnetite contains more Fe(II) sites, leading to a slightly higher bulk saturation magnetisation $\left(\mu_{0} \mathrm{M}_{\mathrm{s}}=0.56 \mathrm{~T}[50]\right)$ than that of maghemite $\left(\mu_{0} \mathrm{M}_{\mathrm{s}}=0.50 \mathrm{~T}\right.$ [195]).

Iron particles collected by magnetic separation were not characterised by TEM as part of this project. However, such characterisation had already been performed by co-workers, on particles produced under comparable conditions. This work is reproduced in Figure 58. From the images, a mean particle diameter of (19.9 \pm 4.3$) \mathrm{nm}$ was estimated. Electron diffraction experiments performed by Cheong [39] suggest that most of the particles are composed of either $\mathrm{Fe}$ or $\mathrm{Fe}_{3} \mathrm{C}$. It is difficult to discount the possibility that other phases of iron carbide are also present, as the diffraction patterns are very similar. However, $\mathrm{Fe}_{3} \mathrm{C}$ is the most stable phase, meaning these other phases should be no more than minor products. The TEM images show an oxide shell approximately $5 \mathrm{~nm}$ in thickness forms around both particle types. 

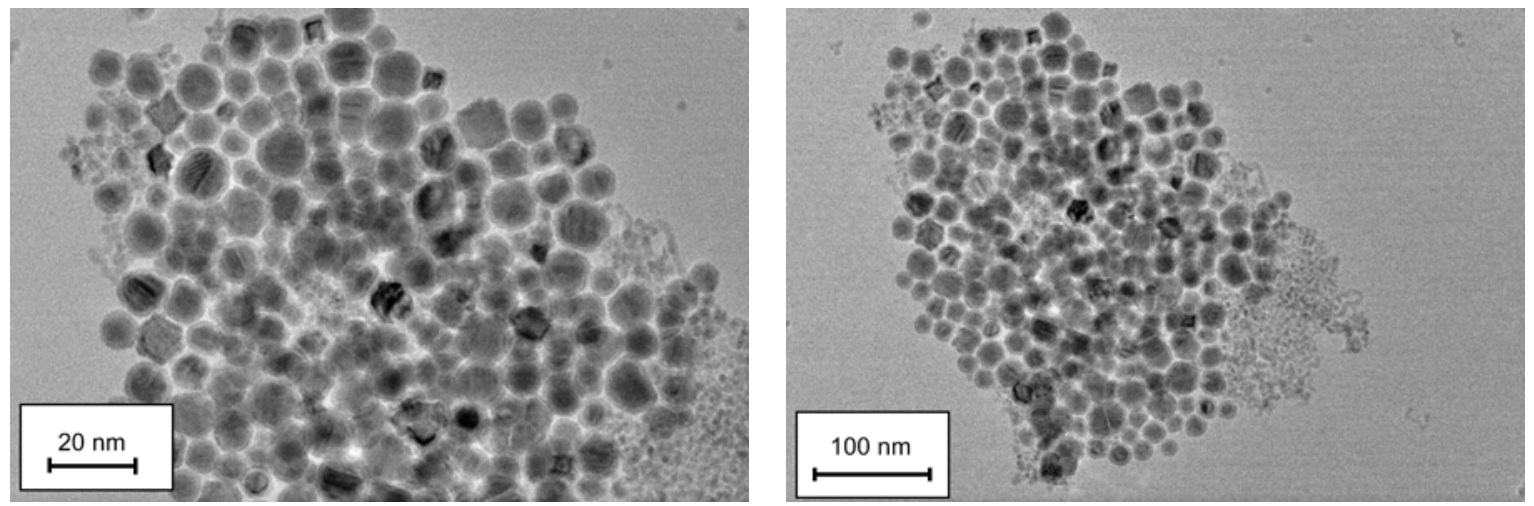

Figure 58: TEM micrographs of iron and iron carbide nanoparticles produced by thermal decomposition.

Smaller iron oxide nanoparticles are also visible.

\subsubsection{Magnetic properties}

The magnetic properties of the nanoparticles were characterised using both SQUID and VSM measurements. VSM enabled the hysteresis curves of the material to be measured, from which the coercivity, remanence and saturation magnetisation could be extracted. SQUID also enabled the temperature dependence of the magnetisation to be examined, which is particularly useful for examining superparamagnetic particles. For a more complete explanation of the operating principles of these techniques, the reader is referred to Sections 3.4.1 and 3.4.2.

The magnetisation curve of the iron and iron carbide nanoparticles is shown in Figure 59. It is clear that the material possesses a finite coercivity and remanence, and that magnetic saturation is achieved within the applied field range of $0.9 \mathrm{~T}(72000 \mathrm{~A} / \mathrm{m})$. This suggests that the particles are ferromagnetic, rather than superparamagnetic. Superparamagnetic particles would not show the hysteresis observed, and would usually require significantly higher fields for saturation to be reached. The key properties obtained from the VSM data are listed in Table 3. A permanent magnet produces a flux density of over $0.3 \mathrm{~T}(24000 \mathrm{~A} / \mathrm{m})$, which is sufficient for these particles to reach saturation. Therefore, it is reasonable to use the high-field approximation (see Equation (27)) when predicting the motion of the particles in the applied field. 


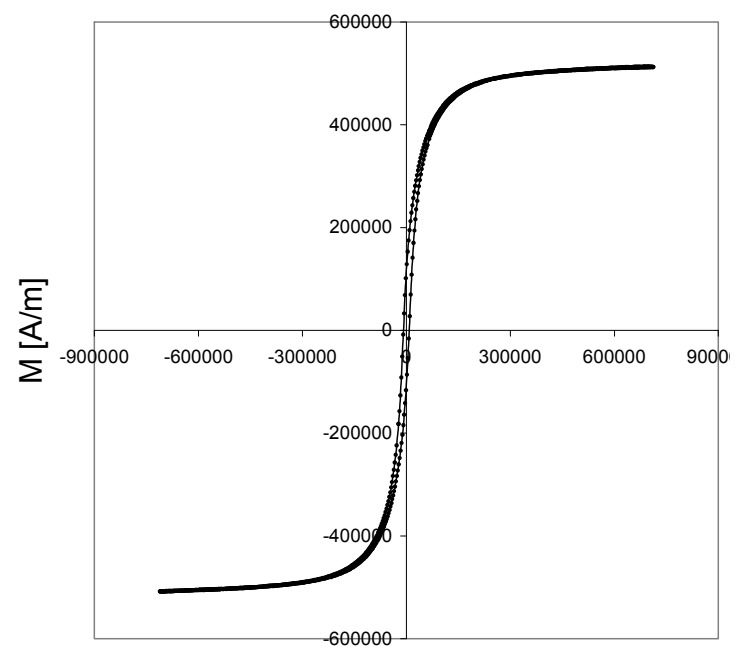

$\mathrm{H}[\mathrm{A} / \mathrm{m}]$

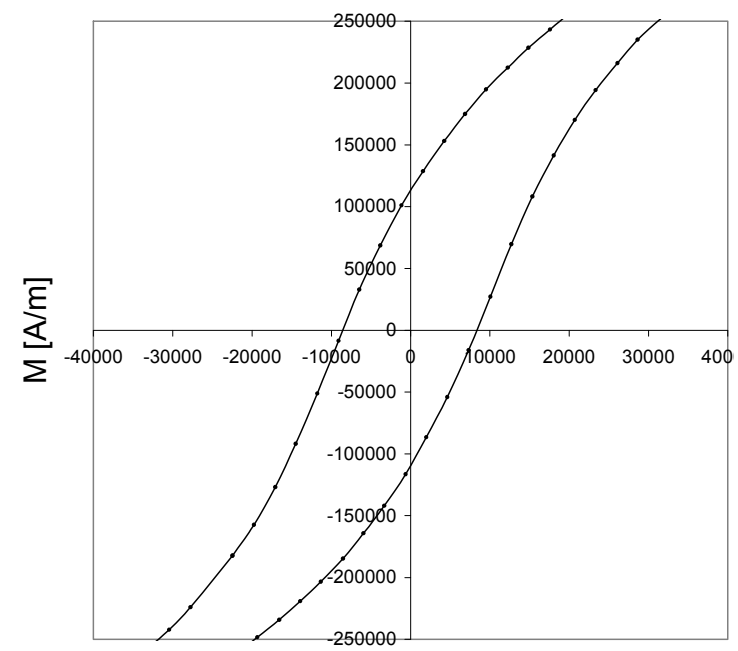

$\mathrm{H}[\mathrm{A} / \mathrm{m}]$

Figure 59: The magnetisation curve for iron/iron carbide nanoparticles. Curves are shown for applied fields up to $0.9 \mathrm{~T}$ (left) and $0.04 \mathrm{~T}$ (right). An iron density of $7.874 \mathrm{gcm}^{-3}$ was assumed when converting to SI units.

\begin{tabular}{|c|c|}
\hline Saturation magnetisation, $M_{s}$ & $5.14 \times 10^{5} \mathrm{~A} / \mathrm{m}$ \\
\hline Coercivity, $H_{c}$ & $8.59 \times 10^{3} \mathrm{~A} / \mathrm{m}$ \\
\hline Remanence, $M_{r}$ & $1.08 \times 10^{5} \mathrm{~A} / \mathrm{m}$ \\
\hline
\end{tabular}

Table 3: Magnetic properties of iron/iron carbide nanoparticles. Values extracted from Figure 59.

The magnetisation curve of the iron oxide nanoparticles is shown in Figure 60. The hysteresis loop of the material is essentially closed, and at low fields the magnetisation is roughly linear. These two properties are characteristic of superparamagnetic particles. We can see in the right-hand curve of Figure 60 that there is an apparent gap in the loop, which is not consistent with superparamagnetism. The small density of measurement points, combined with the asymmetry about the magnetisation axis, prevents a clear conclusion being made. Data at low fields was collected at a much larger sweep rate for this sample than for the iron core-shell particles, which may increase scatter in measurements of the applied field. It is also likely that a small proportion of ferromagnetic contaminants were left behind after the purification step. The key properties measured from the VSM data are listed in Table 4. It should be noted that although a value for the saturation magnetisation has been quoted, the particles are clearly unsaturated within the field range used. This slow approach to saturation is 
commonly seen in paramagnetic materials (see Section 2.1.1), and occurs due to thermal disruption of the dipole moments. This behaviour is often observed in superparamagnetic particles as well, with the alignment of entire particles being affected in place of atomic moments.
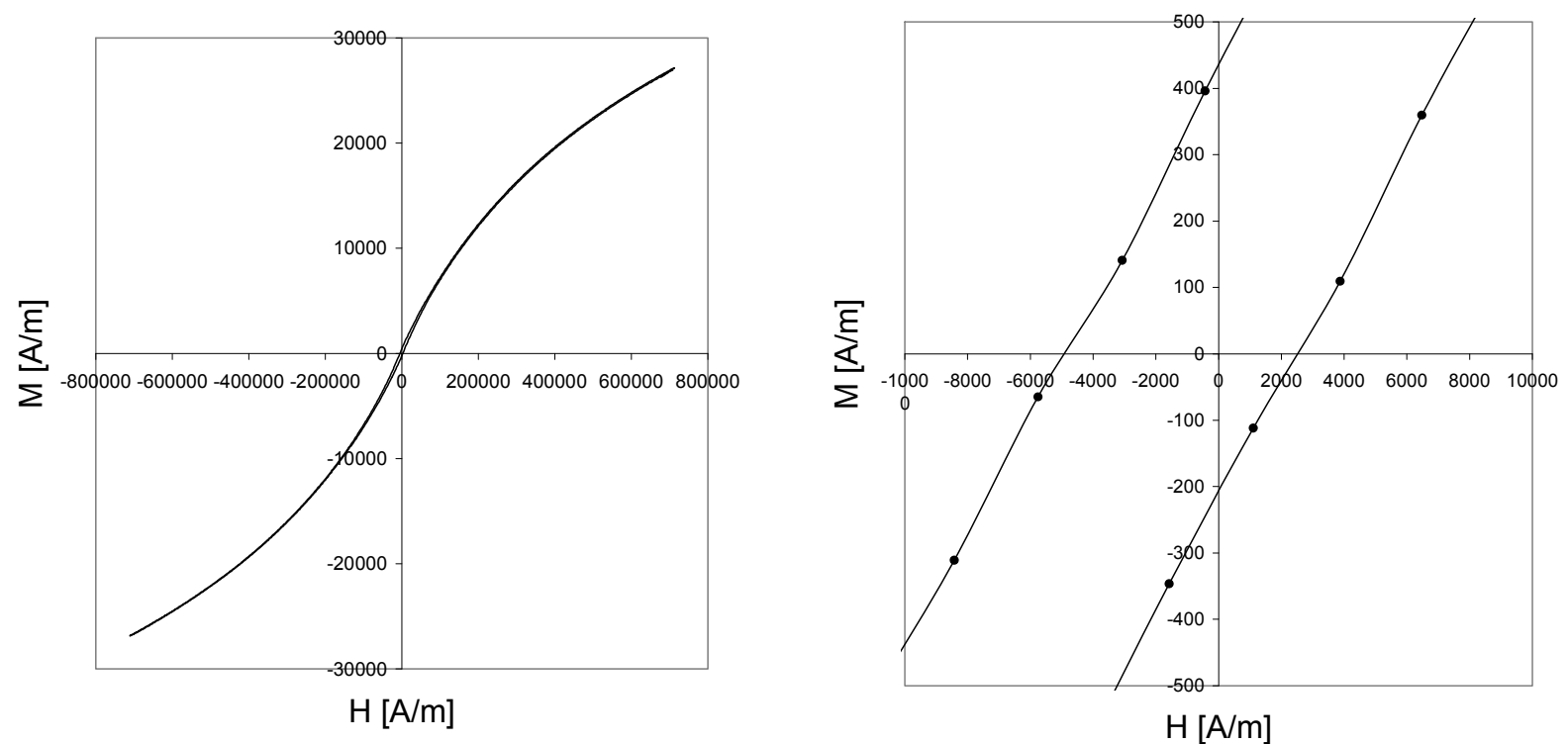

Figure 60: The magnetisation curve for iron oxide nanoparticles. Curves are shown for applied fields up to $0.9 \mathrm{~T}$ (left) and $12.5 \mathrm{mT}$ (right). A density for magnetite of $5.175 \mathrm{~g} / \mathrm{cm}^{3}$ was used when converting to SI units.

\begin{tabular}{|c|c|}
\hline Saturation magnetisation, $M_{s}$ & $2.72 \times 10^{4} \mathrm{~A} / \mathrm{m}$ \\
\hline Coercivity, $H_{c}$ & $3.58 \times 10^{3} \mathrm{~A} / \mathrm{m}$ \\
\hline Remanence, $M_{r}$ & $4.25 \times 10^{2} \mathrm{~A} / \mathrm{m}$ \\
\hline
\end{tabular}

Table 4: Magnetic properties of iron oxide nanoparticles. Values extracted from Figure 60.

Clarification as to whether each particle type is ferromagnetic or superparamagnetic may be gained by looking at the temperature dependence of the magnetisation. As discussed in Section 2.1.4, superparamagnetism is a temperature-dependent effect. The blocking temperature, $T_{B}$, is the temperature at which superparamagnetism sets in. The zero-field cooling (ZFC) and field-cooling (FC) magnetisation curves measured for magnetite particles are shown in Figure 61. Both curves were produced by gradually increasing the temperature in the presence of a small applied field $(H=2.5 \mathrm{mT})$ and recording the magnetic moment. However, the curves differ in the manner in which the sample was first cooled. The FC sample was cooled from room temperature with the field switched on, meaning a permanent magnetisation was induced in the particles. The ZFC sample 
was cooled in the absence of a field, so a superparamagnetic sample would start the run with no net initial magnetisation. Once all particles are unblocked, the ZFC and FC curves will overlap, as the coercivity and remanence both vanish. It is important to note that $T_{B}$ is simply the temperature above which most of the particles are superparamagnetic; some particles make the transition at temperatures above or below this value.

Lu et al. [196] suggested that the derivative of the difference between the ZFC and FC curves gives the distribution of blocking temperatures. This distribution is overlaid on Figure 61, and suggests a dominant blocking temperature of $87 \mathrm{~K}$. A second estimate of $107 \mathrm{~K}$ was obtained by considering the temperature at which the curves essentially meet, indicating almost all the particles are unblocked. Both these values are far below room temperature, confirming that the particles were superparamagnetic under normal conditions.

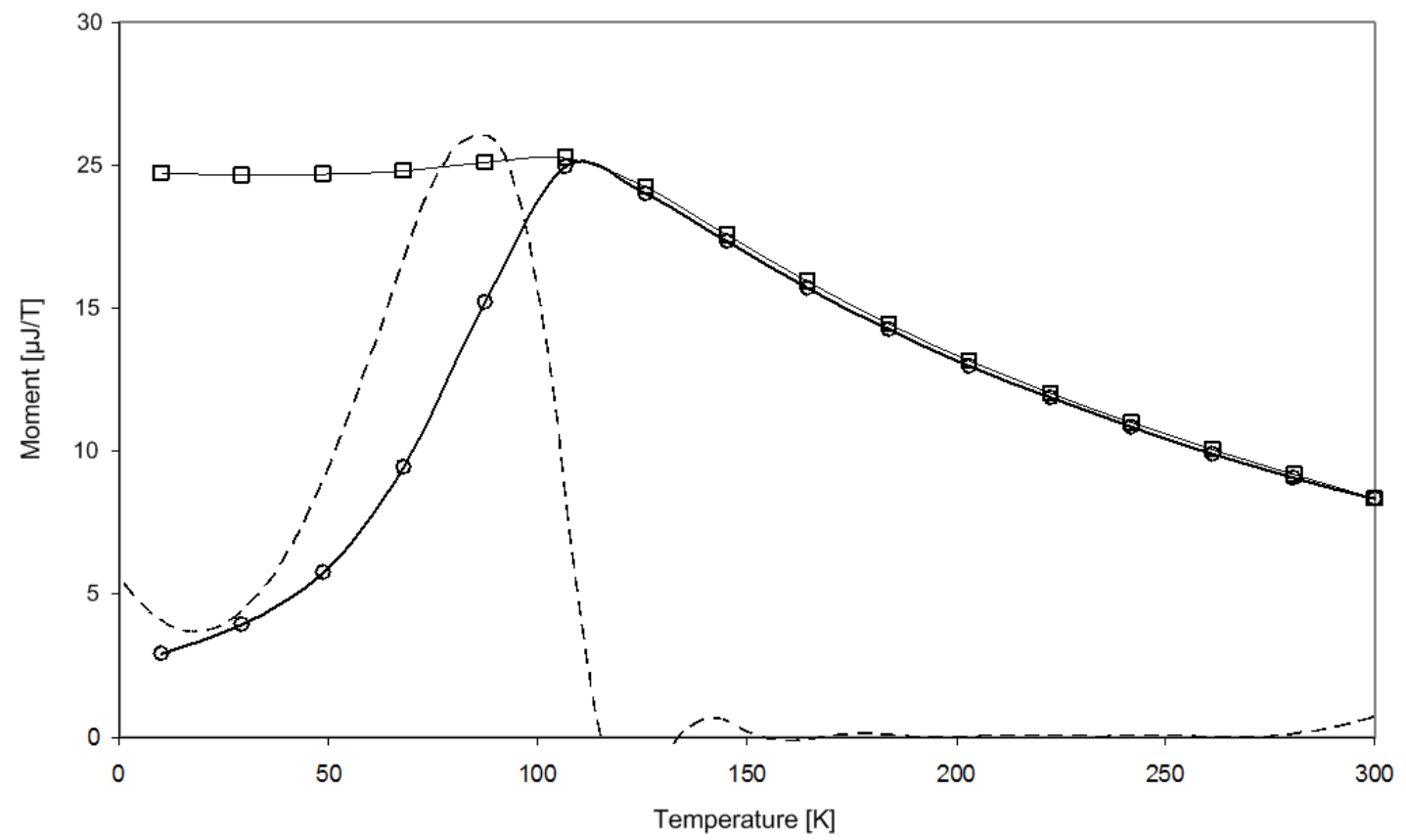

Figure 61: Magnetic moments of the iron oxide nanoparticles, as a function of temperature. Shown is the dependence after first cooling in a $2.5 \mathrm{mT}$ field $(\mathrm{FC}, \square)$ and in zero field $(\mathrm{ZFC}, \circ)$. The $1^{\text {st }}$ derivative of the FC-ZFC represented by a dashed line.

The Arrhenius law, expressed in Equation (8), relates the blocking temperature to particle size. For simplicity, we assume that the only magnetic anisotropy the particles 
experience is the magnetocrystalline component. Equation (8) may then be rewritten in terms of the diameter of a spherical particle as

$$
a \approx\left[\frac{6 k T}{\pi K} \ln \left(\frac{\tau_{m}}{\tau_{0}}\right)\right]^{\frac{1}{3}},
$$

where $a$ is the particle diameter, $K$ is the magnetocrystalline anisotropy energy density, $k$ is Boltzmann's constant and $T$ is the temperature. $\tau_{0}$ is the 'attempt time' of the material, assumed to be $10^{-9}$ seconds (as stated in Section 2.1.4), while $\tau_{m}$ is the time taken for the magnetic measurement, assumed to be 100 seconds. Fortunately, the estimated diameter is relatively insensitive to the exact values of $\tau_{0}$ and $\tau_{m}$.

We may now use the blocking temperature distribution seen in Figure 61 to obtain a distribution of particle sizes, by using Equation (37) to convert values of blocking temperature to the equivalent particle diameters. The conversion requires us to assume a value for the magnetocrystalline anisotropy constant $K$. A value for magnetite of $1.10 \times 10^{4} \mathrm{Jm}^{-3}$ [197] was therefore used. The distribution, as displayed in Figure 62, has a peak at $17.5 \mathrm{~nm}$. This is significantly greater than the value measured by TEM imaging of $(4.8 \pm 1.2) \mathrm{nm}$. The most likely source of this discrepancy is that the assumed value of the anisotropy is too low. If the surface anisotropy of the particles was known, this would be expected to increase the energy barrier, and hence reduce the mean particle size estimate. 


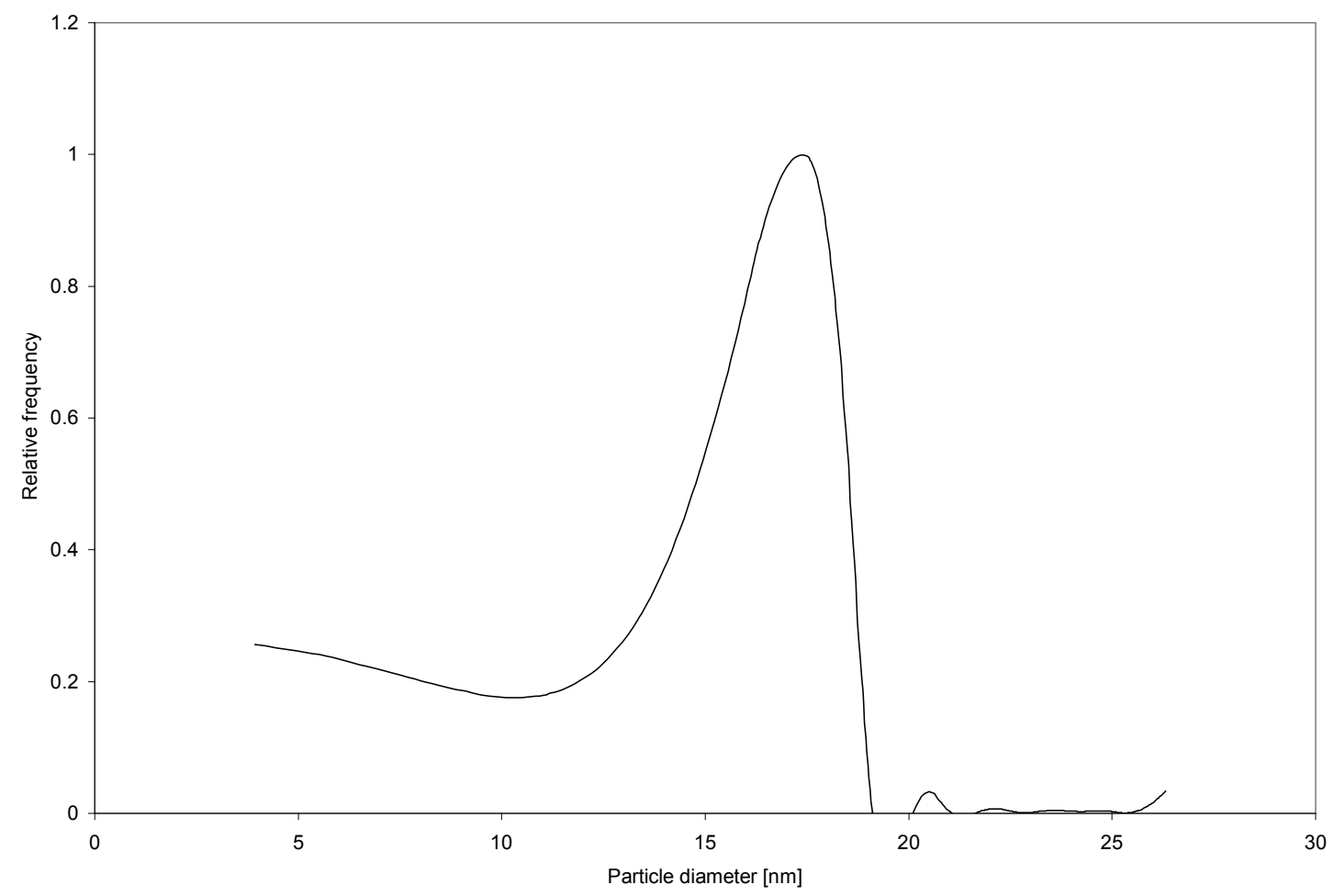

Figure 62: Size distribution for iron oxide particles, calculated from data in Figure 61 using Equation (37). The magnetocrystalline anisotropy was assumed as being that of magnetite, $K=1.10 \times 10^{4} \mathrm{Jm}^{-3}$.

Having confirmed that the iron oxide nanoparticles were superparamagnetic, we could obtain a second estimate of the mean particle size by fitting the form of the Langevin equation for paramagnets (see Equation (4)) to the room-temperature magnetisation data. Figure 63 shows the results of such a fit to the VSM magnetisation data, performed using MATLAB. From the fit, values for the saturation magnetisation of $M_{s}=3.38 \times 10^{4}$ $\mathrm{A} / \mathrm{m}$ and Langevin coefficient of $\alpha=5.84 \times 10^{-6} \mathrm{~m} / \mathrm{A}$ were extracted. Equation (10) then relates $\alpha$ to the particle volume, provided the saturation magnetisation of the bulk material is known. For magnetite this is $\mu_{0} M_{s}=0.56 \mathrm{~T}$ [50], which results in a spherical particle diameter of $4.34 \mathrm{~nm}$. This agrees within uncertainty with the value of $4.8 \pm 1.2$ $\mathrm{nm}$, measured directly by TEM. 


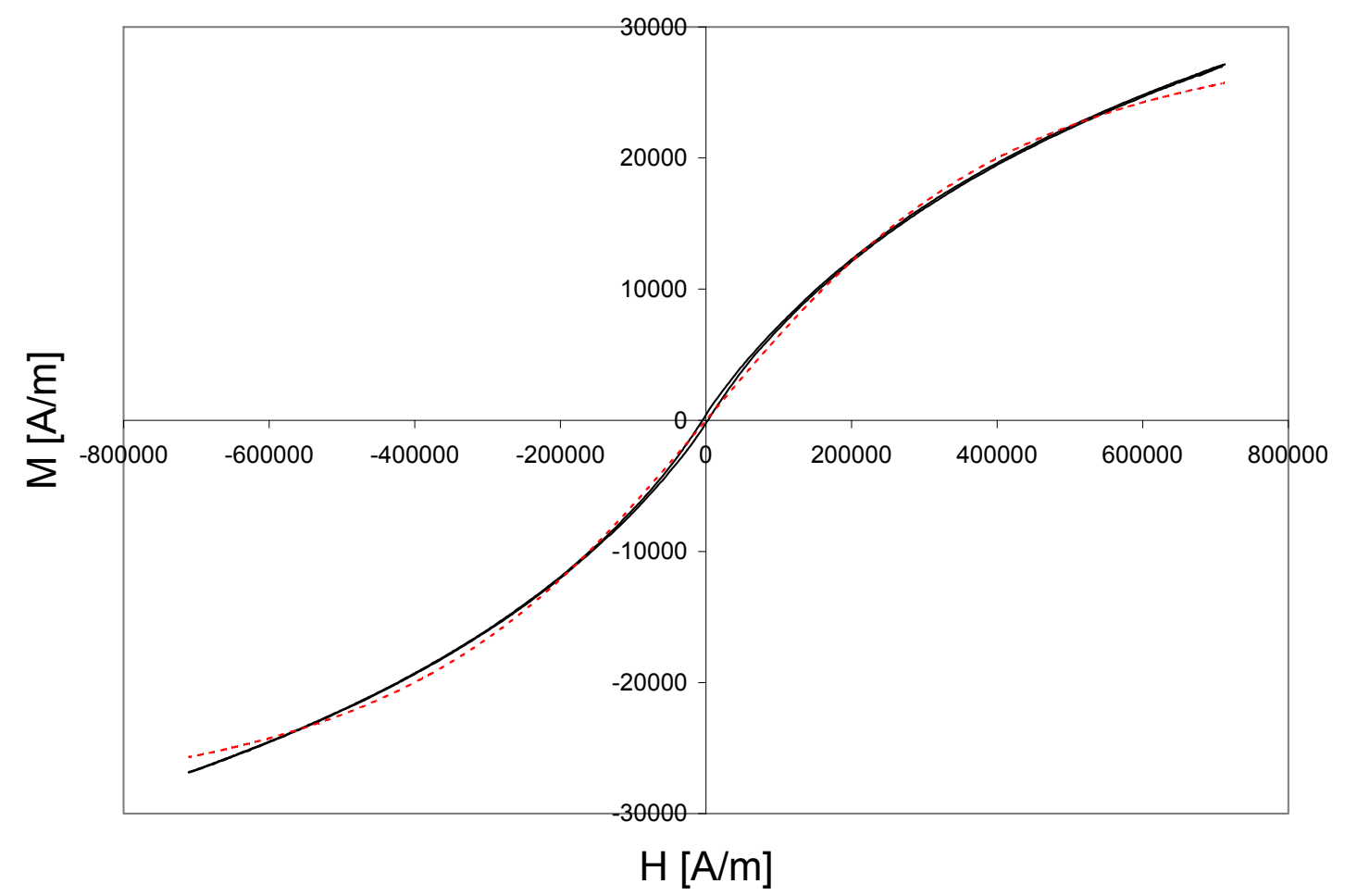

Figure 63: VSM magnetisation data for iron oxide nanoparticles, with Langevin nonlinear fit (red dashed line). Curve fitting was performed using MATLAB.

\subsection{Properties of commercial microbeads}

\subsubsection{Size and morphology}

Three different types of magnetic microbeads were used to varying degrees during this project. It is important to have knowledge of the size and shape of each type, as this will strongly affect the significance of fluid drag, and therefore the velocity of the particles in an applied field. The synthesis methods used by the manufacturers are expected to produce monodisperse particles, meaning a solution containing a single bead type should give predictable behaviour in an applied field. If the particles are polydisperse, some particles will travel much more slowly than others, making separation more difficult.

Images were obtained using optical microscopy. Samples were prepared by placing a few drops of solution on a glass slide using a pipette, and allowing the solvent to evaporate. Images were collected using transmitted rather than reflected light, as this generated stronger contrast. 
Micrographs of Spherotech PM-20-10 and PM-50-10 magnetic beads are shown in Figure 64 . The close packing of the particles suggests that they are highly monodisperse. Diameters of $(4.5 \pm 0.5) \mu \mathrm{m}$ and $(2.7 \pm 0.3) \mu \mathrm{m}$ were measured for the PM-50-10 and PM20-10 particles respectively. These values agree within uncertainty with nominal values of 5.0-5.9 $\mu \mathrm{m}$ and 2.0-2.9 $\mu \mathrm{m}$ reported by the manufacturer [194]. In the images the particles appear as bright spots with a dark outline. This outline is thought to be due to poorer light transmission at the edges of the particles, meaning the thicker outlines on the $5 \mu \mathrm{m}$ particles is due to their increased vertical height. Particle diameters were only measured out to the midpoint of the outline, possibly explaining the underestimate of size for these particles.
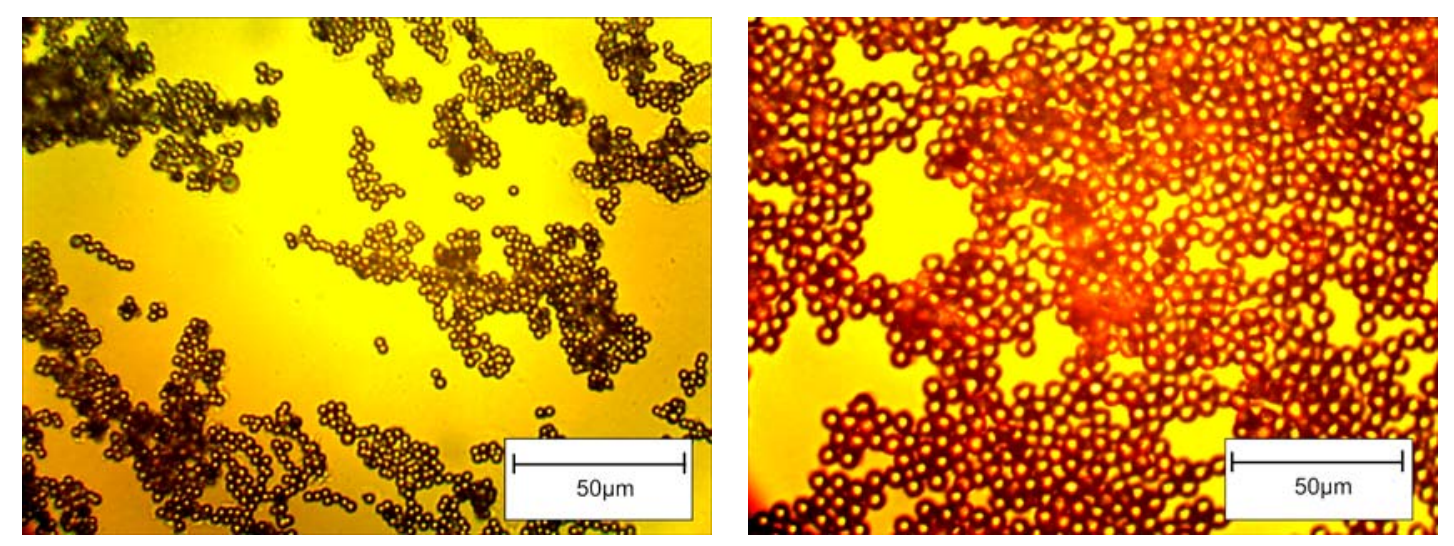

Figure 64: Micrographs of Spherotech PM-20-10 (left) and PM-50-10 magnetic microbeads. Images collected at 60x magnification.

A micrograph of Dynabead MyOne magnetic beads is shown in Figure 65. From the image the bead diameter is estimated as $(1.05 \pm 0.12) \mu \mathrm{m}$. This value is in excellent agreement with reported values of $1.05 \mu \mathrm{m}$ obtained by Fonnum et al. through SEM analysis [45]. 


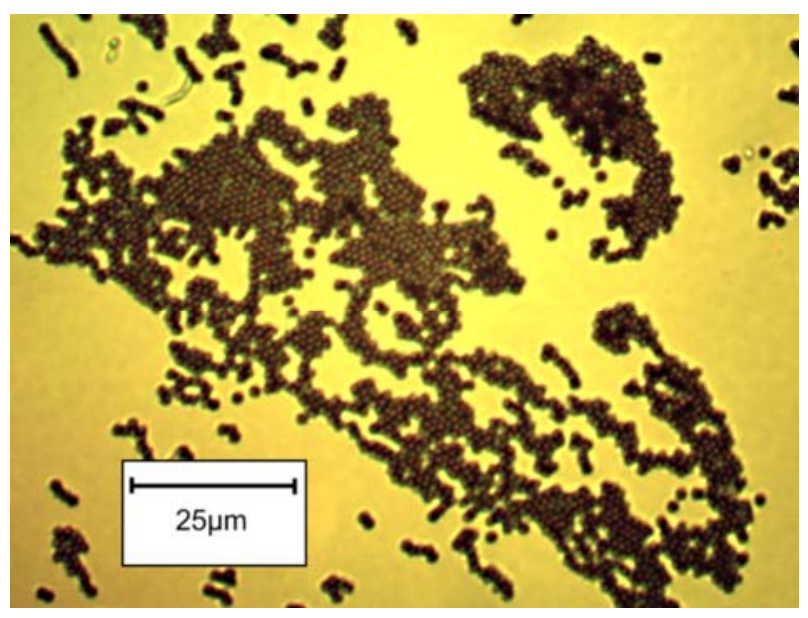

Figure 65: Micrograph of Dynabead MyOne magnetic microbeads at 60x magnification.

\subsubsection{Magnetic properties}

Superparamagnetic microbeads used in this project were characterised by VSM. Samples included Spherotech PM-20-10 (2.5 $\mu \mathrm{m})$ and PM-50-10 (5.5 $\mu \mathrm{m})$ beads, as well as Dynabeads MyOne $(1.05 \mu \mathrm{m})$ beads. This served to confirm the properties reported by the manufacturers and other researchers. Beads from both manufacturers consist of an ensemble of superparamagnetic iron oxide nanoparticles, dispersed in a polymer matrix. As such, data from the magnetisation curves may be used to estimate the sizes of the embedded nanoparticles, but not the sizes of the beads themselves.

\section{MyOne beads from Invitrogen}

The magnetisation data for Dynabeads MyOne particles is shown in Figure 66, and shows clear evidence of superparamagnetism. Key properties from the data are summarised in Table 2. The coercivity of the beads is almost an order of magnitude smaller than that of the synthesised iron oxide nanoparticles measured in Section 4.1.2 (see Table 4). It is interesting that even though the beads were superparamagnetic, they were readily saturated in a 1 tesla field. This is in stark contrast to the behaviour of the iron oxide nanoparticles in Figure 60, which showed a much slower approach to saturation. 

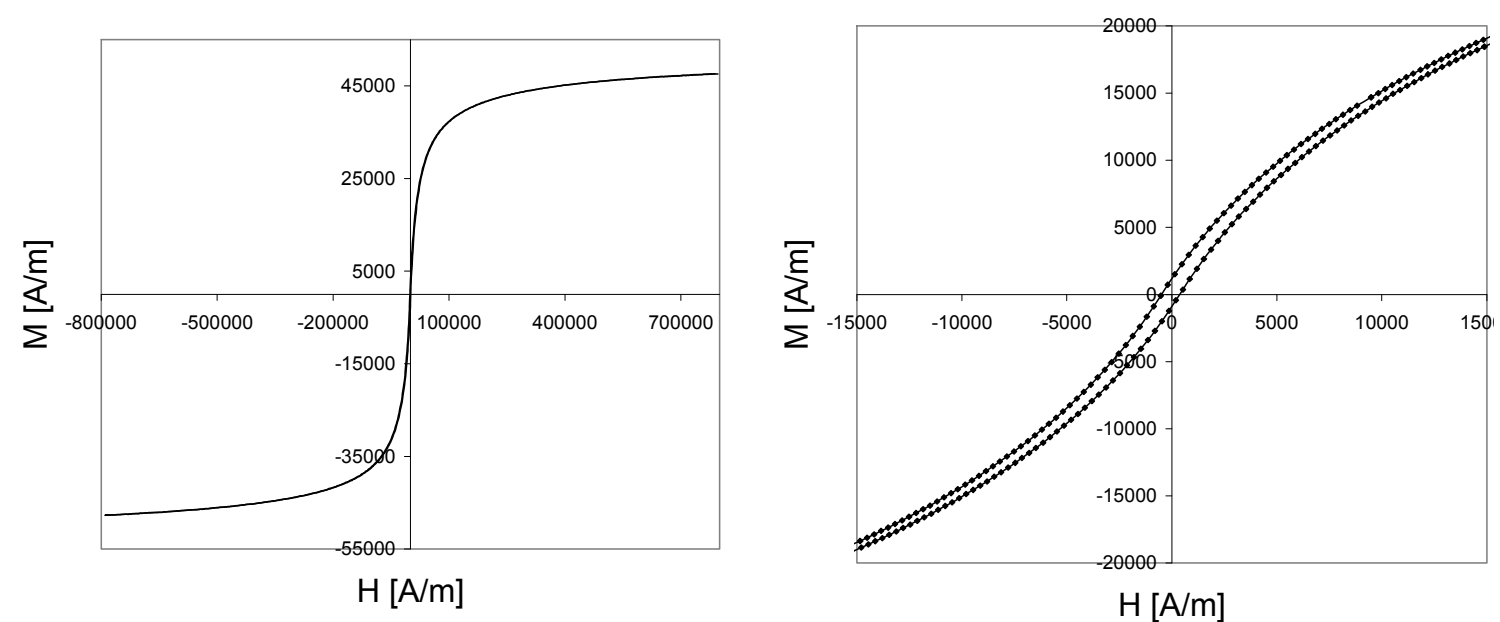

Figure 66: VSM magnetisation data for Dynabeads MyOne $1.05 \mu \mathrm{m}$ microbeads. Curves are shown for applied fields up to $1 \mathrm{~T}$ (left) and $0.02 \mathrm{~T}$ (right). A bead density of $1.7 \mathrm{~g} / \mathrm{cm}^{3}$ [45] was used for conversion to SI units.

\begin{tabular}{|c|c|}
\hline Saturation magnetisation, $M_{s}$ & $4.76 \times 10^{4} \mathrm{~A} / \mathrm{m}$ \\
\hline Coercivity, $H_{c}$ & $4.25 \times 10^{2} \mathrm{~A} / \mathrm{m}$ \\
\hline Remanence, $M_{r}$ & $1.05 \times 10^{3} \mathrm{~A} / \mathrm{m}$ \\
\hline
\end{tabular}

Table 5: Magnetic properties of Dynabeads MyOne microbeads. Values extracted from Figure 66.

The mean size of the nanoparticles present in the microbeads was estimated by fitting the form of Equation (4) to the magnetisation data in Figure 66. This was conducted in MATLAB in an identical manner to that described in Section 4.1.2. From the fit in Figure 67, a saturation magnetisation of $M_{s}=4.722 \times 10^{4} \mathrm{~A} / \mathrm{m}$ and Langevin coefficient of $\alpha=5.938 \times 10^{-5} \mathrm{~m} / \mathrm{A}$ were extracted. Fonnum et al. [45] used Mossbauer spectroscopy to determine that the beads contain maghemite, which has a saturation magnetisation of $\mu_{0} M_{s}=0.50 \mathrm{~T}$ [195]. Using this information with Equation (10) gave a mean particle diameter of $9.77 \mathrm{~nm}$. This is larger than the average value of $7.5 \mathrm{~nm}$ obtained from Xray diffraction and magnetic analysis by Fonnum et al. [45]. In their work they suggested that the nanoparticles in each bead tend to form clusters up to $20 \mathrm{~nm}$ in size, which could lead to an overestimate of the individual particle size if the particles interact. 


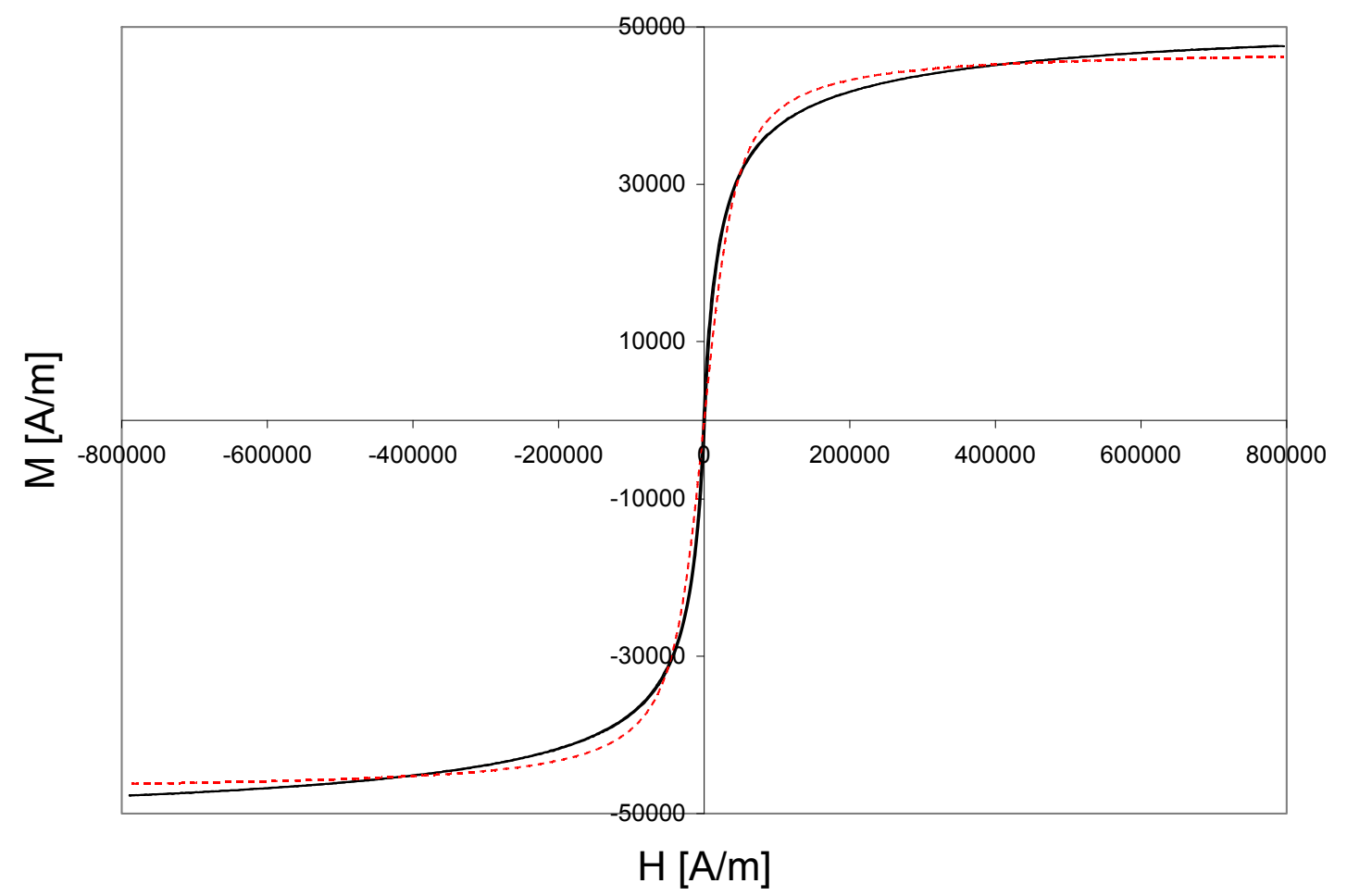

Figure 67: VSM magnetisation data for Dynabead MyOne microbeads, with Langevin nonlinear fit (red dashed line). The fit was performed using MATLAB.

\section{PM-20-10 and PM-50-10 beads from Spherotech}

The magnetisation curves for PM-20-10 and PM-50-10 beads supplied by Spherotech are shown in Figure 68. Key parameters obtained from the curves are shown in Table 6. The particles show superparamagnetic behaviour, as claimed by the manufacturer, although both bead sizes exhibit a slight hysteresis. The coercivity is similar between the two bead sizes, which is to be expected as they should contain the same magnetic material. The fundamental difference between the two bead types is that the saturation magnetisation of the $2 \mu \mathrm{m}$ beads is considerably larger than that of the $5 \mu \mathrm{m}$ beads. This suggests that the smaller beads have a larger magnetite content. The manufacturer does not directly state the magnetite content of the beads, but claims that it is between 10 and $15 \%$ [198]. Given that the saturation magnetisation should scale directly with the magnetite content, the observed ratio in $M_{s}$ is consistent with the $2 \mu \mathrm{m}$ beads having $15 \%$ magnetite content and the $5 \mu \mathrm{m}$ beads $10 \%$. As discussed in Section 2.4.2, smaller particles experience increased fluid drag compared to the magnetic force. This makes it necessary to increase their magnetic content to avoid a reduction in their magnetophoretic mobility. 
In calculating the volume magnetisation for Figure 68, the density had to be calculated for both types of bead, as the manufacturer does not quote particle densities. It was assumed that the particles had $12.5 \%$ magnetite content. The density of magnetite is 5.17 $\mathrm{g} / \mathrm{cm}^{3}$, and the density of the polystyrene is $1.05 \mathrm{~g} / \mathrm{cm}^{3}$. A weighted average thus gives a density of $1.57 \mathrm{~g} / \mathrm{cm}^{3}$ for the beads. However, differences in the saturation magnetisations indicate that the loading of magnetic material is different for each type. It follows that the beads must have different densities. Nonetheless, because the magnetite content is known to be within $10-15 \%$, the difference in densities (and therefore $M_{s}$ ) is no greater than $7 \%$. 

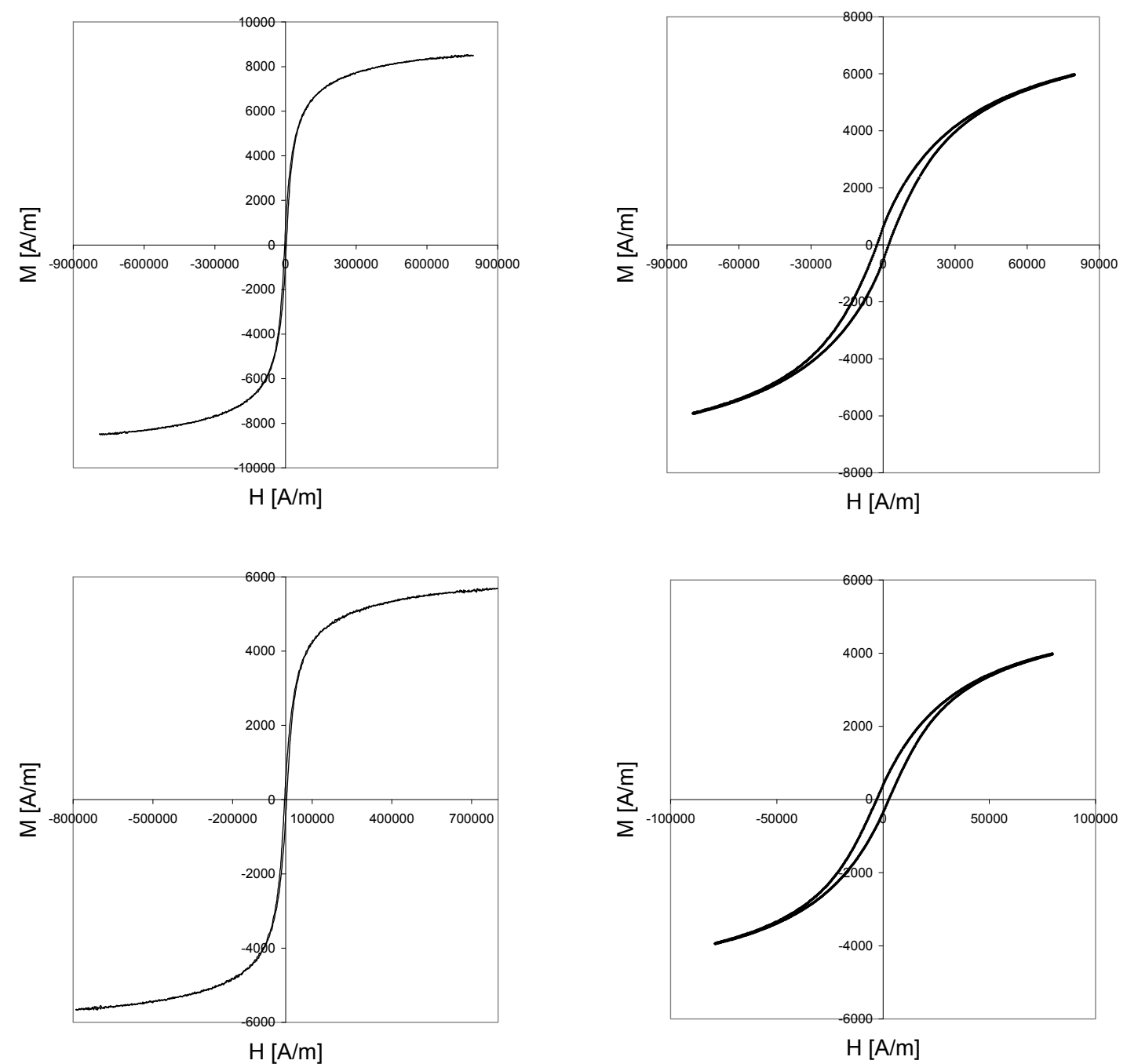

Figure 68: Magnetisation curves for Spherotech superparamagnetic microbeads. Shown are curves for PM-20-10 (top) and PM-50-10 (bottom). Data is shown for ranges of 1 tesla (left) and 0.1 tesla (right). A bead density of $1.57 \mathrm{~g} / \mathrm{cm}^{3}$ was used when calculating the volume magnetisation.

\begin{tabular}{|c|c|c|}
\hline & PM-20-10 & PM-50-10 \\
\hline Saturation magnetisation, $M_{s}$ & $8.54 \times 10^{3} \mathrm{~A} / \mathrm{m}$ & $5.70 \times 10^{3} \mathrm{~A} / \mathrm{m}$ \\
\hline Coercivity, $H_{c}$ & $2.38 \times 10^{3} \mathrm{~A} / \mathrm{m}$ & $2.75 \times 10^{3} \mathrm{~A} / \mathrm{m}$ \\
\hline Remanence, $M_{r}$ & $5.88 \times 10^{2} \mathrm{~A} / \mathrm{m}$ & $3.75 \times 10^{2} \mathrm{~A} / \mathrm{m}$ \\
\hline $\begin{array}{c}\text { Estimated magnetite content } \\
\text { (by volume) }\end{array}$ & $15 \%$ & $10 \%$ \\
\hline
\end{tabular}

Table 6: Magnetic properties of Spherotech microbeads. Values extracted from Figure 68. 


\subsection{Evaluation of Microfluidic Devices}

\subsubsection{Channel reproduction}

The layout of the photomasks used for creating most of the magnetic separation devices is shown in Figure 69. The fluid channels on the mask all have a width of $280 \mu \mathrm{m}$. A slightly different version of this mask was used for creating the Metglas system, which featured recesses for holding the Metglas strips and a channel width of $330 \mu \mathrm{m}$.

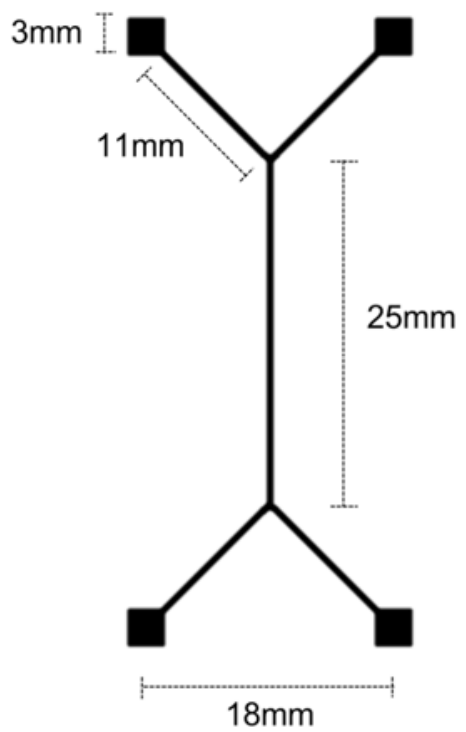

Figure 69: Photomask layout used for creating microfluidic magnetic separation devices, with important dimensions labelled.

Figure 70 shows a master fabricated on a PMMA wafer, using SU-8 photoresist and the above photomask design. The photomask used to create the master was shown in Section 3.1.1, and it is clear that the main features of the mask are reproduced in the SU8. In particular, the junctions and reservoirs are well-resolved, although sharp edges have become rounded. The channel walls also show some roughness, which is likely to be due to the lack of contrast at the feature boundaries in the photomask. Use of an improved printing process would be expected to reduce this effect. 

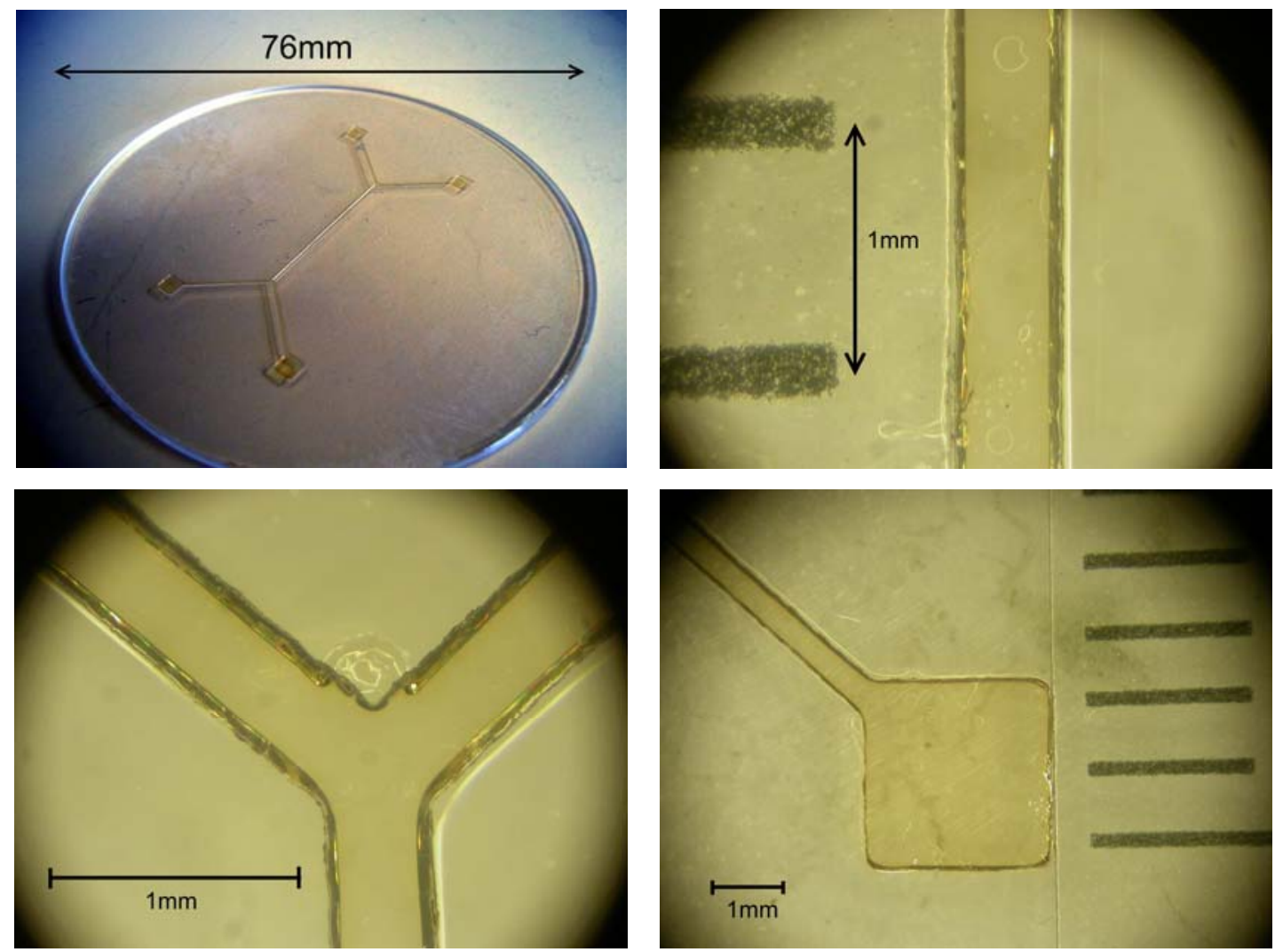

Figure 70: Images of SU-8 channels defined on a PMMA wafer using photolithography. Top left: $76 \mathrm{~mm}$ diameter wafer with SU-8 channel. Top right: Central channel. Bottom left: Channel junction. Bottom right: Reservoir for fluid exchange with luer interconnector.

From the figure, the width of the main channel is measured as $330 \mu \mathrm{m}$, which is significantly larger than the width of $280 \mu \mathrm{m}$ defined in the photomask. It has been proposed by Chooi [152] that this is caused by the use of an uncollimated UV light source used for the lithography process, as illustrated in Figure 71. The finite thickness of the SU-8 layer creates a gap between the photomask and the bottom layer of photoresist, meaning that UV light not entering normal to the photomask surface may interact with resist that should have remained unexposed. This leads to a tapered crosssection, where the channel is wider close to the PMMA/SU-8 interface. We would also expect the increase in width to be most severe for high-viscosity photoresists, as these create the thickest layers to expose. Roughness in the SU-8 surface will also worsen the effect, as this will create an air gap between the mask and the resist. 


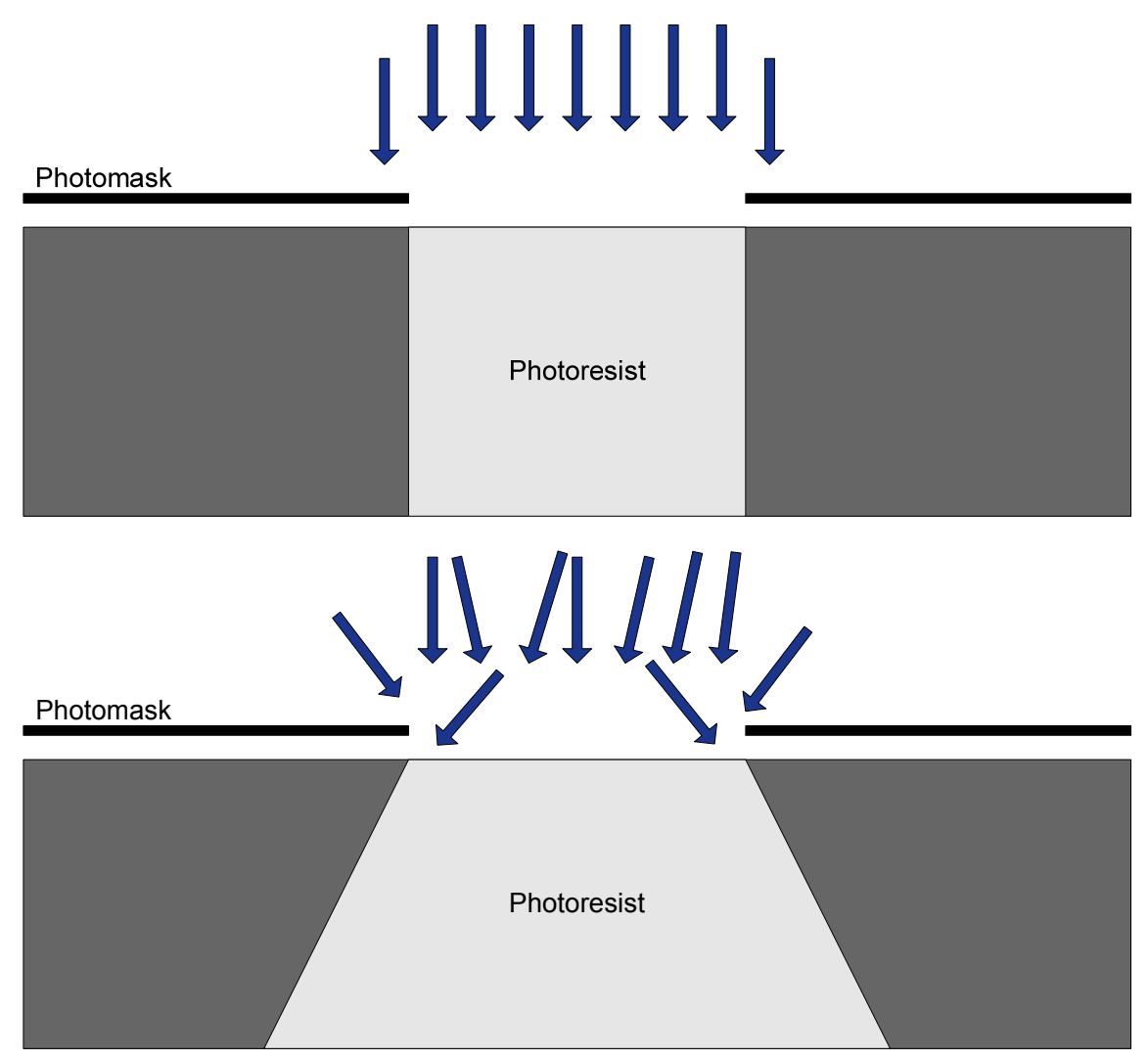

Figure 71: Schematic showing the effect of exposing SU-8 photoresist to UV light through a photomask [152]. Collimated light (top) will accurately reproduce the mask features, while poorly collimated light (bottom) will lead to a trapezoidal cross-section.

A number of modifications to the process may be made to reduce tapering of the sidewalls. The manufacturer recommends the use of a long pass filter, to exclude UV wavelengths less than $350 \mathrm{~nm}$ [158]. Chan-Park et al. [199] also found that reducing the UV exposure to around $60-70 \%$ of the standard dose produced vertical sidewalls. Extending the baking time helped to compensate for the underexposure. Chuang et al. [200] also found that a layer of glycerol, or another liquid with a refractive index similar to that of SU-8, could be used to compensate for the air gap between the photomask and photoresist. Mask diffraction provides an ultimate limit on the resolution of channels produced by photolithography, although this effect becomes important only at much smaller feature sizes than were used in this project.

It should be noted that although the device design described above has the narrowest channels created during the project, it is expected that smaller features could be created with no alterations to the procedure. Masters created in the latter stages of the project 
showed strong bonding between the exposed SU-8 and the PMMA wafer, meaning the resist should remain bonded even with a diminished contact area. The resolution of the photomasks will however place a lower limit on the feature widths that may be obtained. As shown in Figure 72, a small amount of toner bleeds into the channels, which reduces the fidelity of the channel at smaller widths. Using a higher resolution printer would be expected to allow photomasks with finer features to be easily produced. The intended application of the device called for relatively wide channels, in order to allow reasonably high flow rates to be used, meaning that there existed little need to create smaller structures.

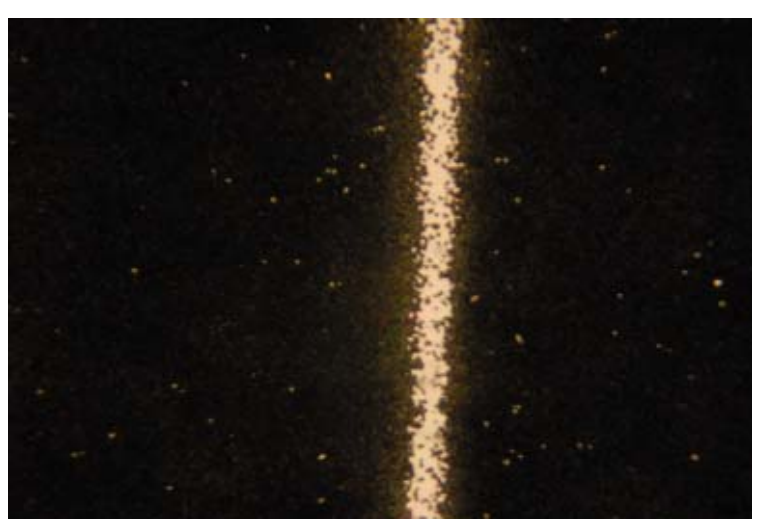

$50 \mu \mathrm{m}$

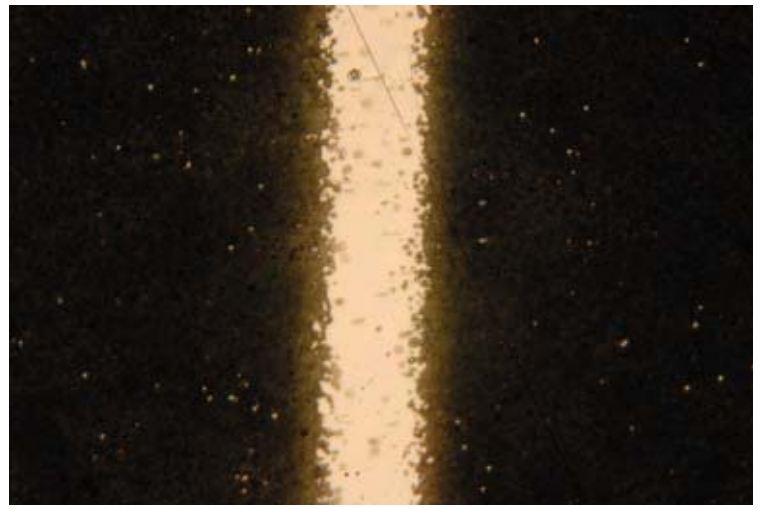

$200 \mu \mathrm{m}$

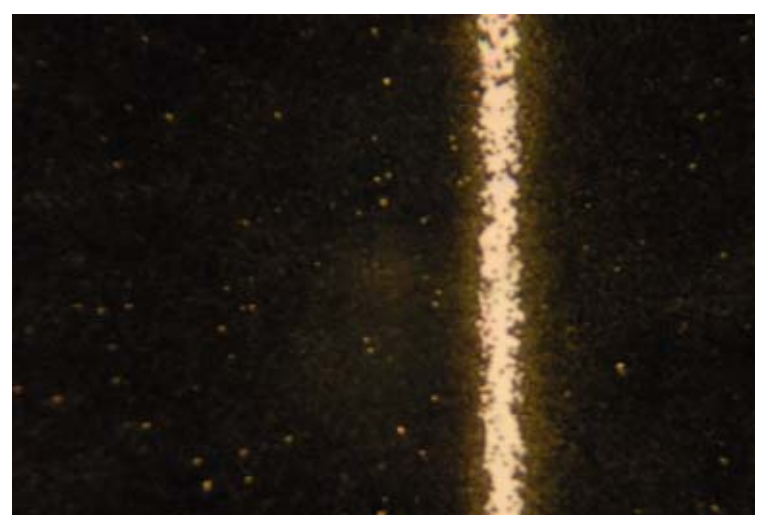

$110 \mu \mathrm{m}$

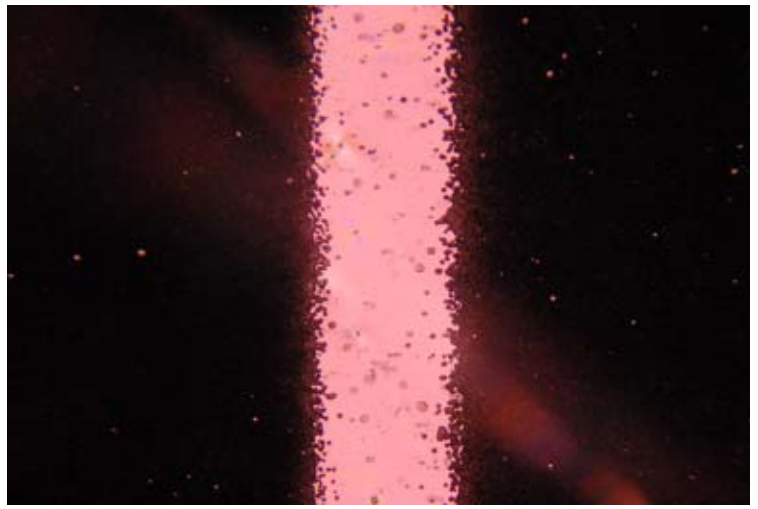

$280 \mu \mathrm{m}$

Figure 72: Microscope images at 40× magnification of transparency photomasks. Captions indicate the intended width of the channel for each photomask. Images reproduced from [152].

\subsubsection{Flow behaviour}

Microfluidic devices fabricated for this project demonstrated laminar flow at a wide range of flow rates. The most dramatic observation of this is shown in Figure 73, in which a solution of food dye was run in parallel with distilled water at flow rates of 50, 200 and $500 \mu \mathrm{L}$ per min. In these photos we can see that the two fluid streams formed a 
sharp interface at the midpoint of the channel when they first came into contact. The widths of the dye and water streams were equal at all flow rates measured. This is predicted by Equation (14), provided both fluids are introduced at the same rate and have the same viscosity (true in this case). It should be noted that the images of Figure 73 were all taken once the flow had stabilised. It was noticed that physical contact with the device would temporarily disrupt the balance in flows, with recovery occurring most rapidly at higher flow rates. It is likely that this effect occurs because the PDMS body of the device is highly compressible, meaning contact is likely to lead to pressure pulses inside the channel.

The interface between the two fluids was noticeably less distinct in images taken further down the channel, with this effect being strongest at lower flow rates. This is consistent with diffusive mixing, which predicts migration of the solute that increases with incubation time, as expressed in Equation (15). As discussed in Section 2.3.3, we do not expect diffusion to be significant in the case of microparticle dispersions, but the small molecules in the food dye will diffuse much more rapidly, making significant mixing possible.

A small amount of dye can be seen exiting the channel through the wrong outlet, at each of the three flow rates used. It is likely that this is mostly dye that has diffused across the flow boundary into the water stream, as the amount of contamination appears to be greatest at low flow rates. At $500 \mu \mathrm{L} / \mathrm{min}$ a faint plume of dye can be seen moving through the middle of the water outlet, rather than along the sidewall. This suggests that small levels of turbulence may be generated near the junction at high flow rates. This turbulence may then force additional dye into the water stream. However, this appears to be a minor effect at the flow rates used. 
$50 \mu \mathrm{L} / \mathrm{min}$
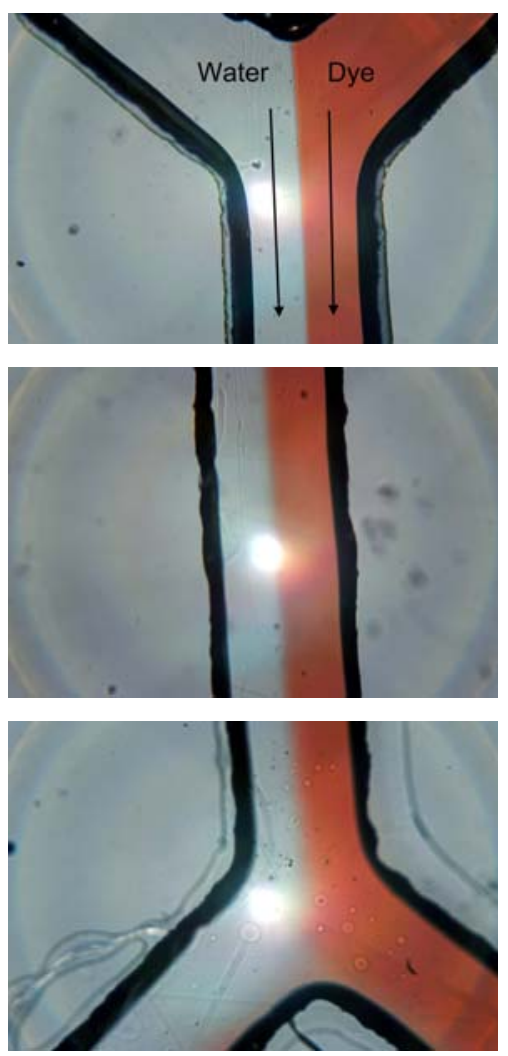

$200 \mu \mathrm{L} / \mathrm{min}$
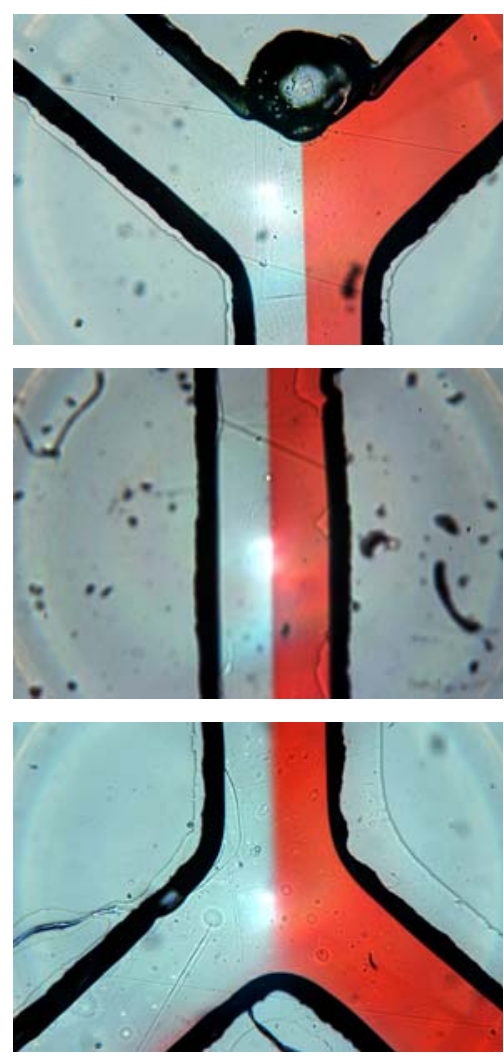

$500 \mu \mathrm{L} / \mathrm{min}$
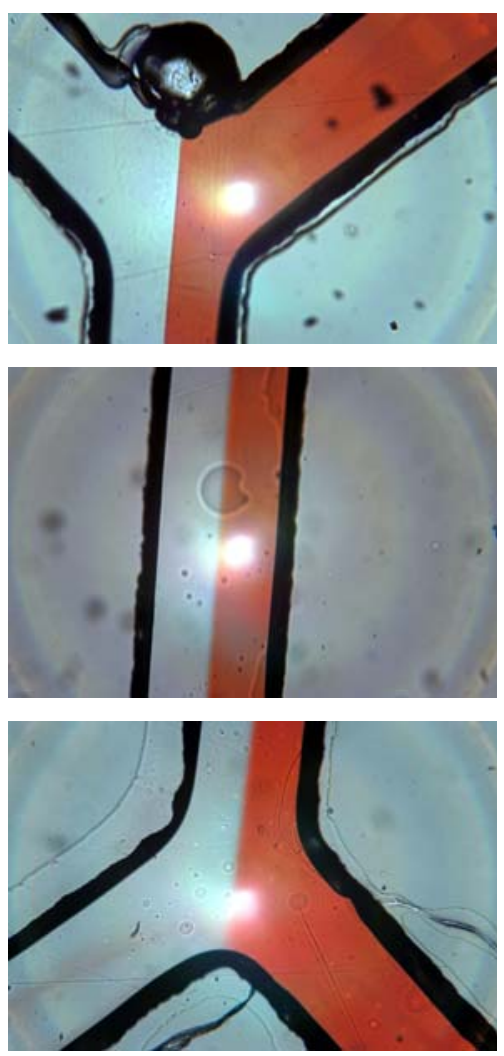

Figure 73: Laminar flow of food dye against distilled water at flow rates of $50 \mu \mathrm{L} / \mathrm{min}$ (left), $200 \mu \mathrm{L} / \mathrm{min}$ (centre) and $500 \mu \mathrm{L} / \mathrm{min}$ (right). Images were taken at the input junction (top), main channel (centre) and outlet junction (bottom), using a $4 \mathrm{x}$ microscope objective.

It was not possible to quantify the level of mixing from visual observations alone, so samples were also collected for UV-visible spectroscopy measurements. A $1.25 \mu \mathrm{L} / \mathrm{mL}$ solution of food dye was used to repeat the above experiments, at flow rates of 200 and $500 \mu \mathrm{L} / \mathrm{min}$. It was shown in Figure 52 that the sample absorbance is linear in concentration when the solution is this dilute. Comparing the absorbance of the solution collected at both outlets therefore provides a measure of the amount of mixing between the fluid streams. The results of this measurement are summarised in Table 7. 


\begin{tabular}{|c|c|c|c|c|c|}
\hline $\begin{array}{c}\text { Experiment } \\
\text { type }\end{array}$ & $\begin{array}{c}\text { Flow rate, } \\
\mu \mathrm{L} / \mathrm{min}\end{array}$ & Outlet & $\begin{array}{c}\text { Absorbance } \\
(\lambda=507 \mathrm{~nm})\end{array}$ & $\begin{array}{c}\text { Concentration, } \\
\mu \mathrm{L} / \mathrm{mL}\end{array}$ & $\begin{array}{c}\text { Concentration, } \\
\%\end{array}$ \\
\hline \multirow{3}{*}{ Mixing } & 200 & Dye & 1.626 & 1.04 & 83 \\
\cline { 2 - 6 } & \multirow{2}{*}{500} & Water & 0.071 & 0.05 & 4 \\
\cline { 2 - 6 } & & Dye & 1.746 & 1.11 & 89 \\
\hline \multirow{2}{*}{ Control } & \multirow{2}{*}{500} & Dye & 0.061 & 0.04 & 3 \\
\cline { 3 - 6 } & & Dye & 1.747 & 1.12 & 89 \\
\hline
\end{tabular}

Table 7: Measurements of mixing between a dye solution $(1.25 \mu \mathrm{L} / \mathrm{mL})$ and water in a microfluidic device. Measurements from a control experiment, where dye was run through both channels, is included for comparison. Measurements were performed using UV-Vis spectroscopy.

The results of Table 7 suggest that a maximum of $4 \%$ of the dye solution was lost into the buffer stream, and that the loss was greatest at the lowest flow rate. We may gain a rough estimate for the level of mixing expected solely due to diffusion using Equation (18). Unfortunately the molecular weight (and hence the diffusion coefficient) for the dye is not known. It has therefore been assumed that the diffusion coefficient is similar to that of sugar molecules, $D \sim 5 \times 10^{-10} \mathrm{~m}^{2} \mathrm{~s}^{-1}$ [4]. Figure 74 shows the expected distribution of dye molecules across the width of the buffer stream, for an elemental volume at the outlet junction of the device. Curves are shown for both flow rates used. 


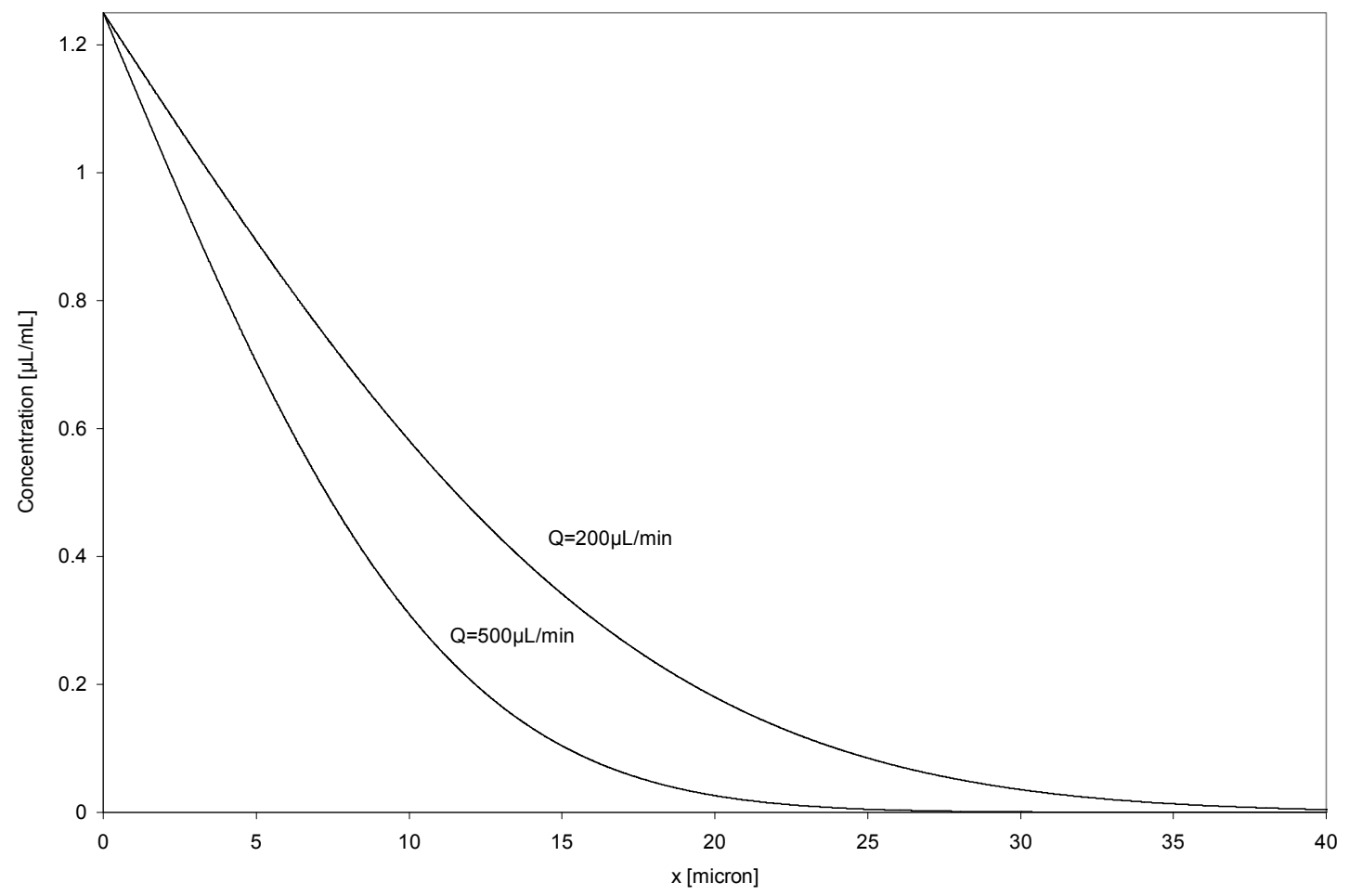

Figure 74: Concentration distribution for dye diffusing from the fluid interface into a buffer stream in a microfluidic channel.

The area under the curves in Figure 74 is directly proportional to the amount of dye transferred. If 100 percent of the dye diffused into the buffer stream, we would expect a constant concentration of $1.25 \mu \mathrm{L} / \mathrm{mL}$ over the full width of the buffer stream. For cases where less diffusion has occurred, we may compare the ratio of the area under the curve to the product of the concentration and the buffer stream width (roughly $165 \mu \mathrm{m}$ ). By this method we predict losses of $6.5 \%$ and $4.2 \%$, for flow rates of 200 and $500 \mu \mathrm{L} / \mathrm{min}$ respectively. These theoretically predicted results are slightly larger than the measured percentages, but similar enough to suggest that diffusion is the primary contributor to fluid mixing in the microchannels.

Two aspects of the results obtained above suggest that additional dilution of the dye occurs. Firstly, at both flow rates the measured dye concentration in the buffer stream is somewhat lower than predicted. Secondly, the percentage concentrations measured at the two outlets of the device sum to approximately $10 \%$ short of unity. It is proposed that this dilution is related to the dead volume of the fluid connectors and tubing. The 
luer interconnectors used each have a dead volume of approximately $100 \mu \mathrm{L}$. The device used was flushed with distilled water before each experiment, meaning that the dye and water solutions will each be diluted by as much as $200 \mu \mathrm{L}$. Each experiment used $3 \mathrm{~mL}$ of dye solution, hence we would expect dilution of around $6 \%$ due to the interconnectors alone. This does not fully explain the observed dilution, but it is also possible that some dilution occurred due to water residues in the tubing. The tubing was flushed with water before each experiment, and while most of this water was ejected from the tubing, a small amount may have remained. However, the level of dilution this would contribute is unknown. Further investigations involved repeating the experiment, but with dye run through both device inlets at once. The concentration profile should be constant across the width of the channel, meaning no net diffusion will occur. In spite of this an $11 \%$ reduction in concentration was observed, consistent with dilution.

In order to determine whether the flow properties observed for the dye may be generalised to a dispersion of particles, the experiment was repeated for a solution of Polysciences $1 \mu \mathrm{m}$ latex beads. Runs were performed at 250 and $500 \mu \mathrm{L} / \mathrm{min}$, using a total volume of $5 \mathrm{~mL}$ to reduce the impact of dead volumes. The results of these runs are summarised in Table 8 . At both flow rates, $2 \%$ of the beads were lost into the buffer solution. Using the same assumptions used for the dye, but with a diffusion coefficient of $D=4 \times 10^{-13} \mathrm{~m}^{2} \mathrm{~s}^{-1}$ (calculated using Equation (18)) we may estimate the loss by diffusion. This gives expected losses of just $0.17 \%$ and $0.12 \%$, meaning the measured change can not be due to diffusion alone.

Transient imbalances in the pressure applied to the two streams may have caused some mixing to occur. However, the same effect would be expected to have occurred during the dye experiments, for which the same pumping setup was used. A more likely cause of the increased mixing observed is the presence of solid particles in the fluid. Numerical simulations conducted by Kajishima and Takiguchi [201] suggested that particle-laden laminar flows can exhibit localised turbulence. The authors attributed this to the formation of particle clusters, which can impair the damping of eddy currents in the surrounding fluid. Visual evidence of this effect, observed during magnetic separation, is presented in Section 4.4.2. 


\begin{tabular}{|c|c|c|c|c|}
\hline $\begin{array}{c}\text { Flow rate, } \\
\mu \mathrm{L} / \mathrm{min}\end{array}$ & Outlet & $\begin{array}{c}\text { Absorbance } \\
(\lambda=507 \mathrm{~nm})\end{array}$ & $\begin{array}{c}\text { Concentration, } \\
\mu \mathrm{L} / \mathrm{mL}\end{array}$ & $\begin{array}{c}\text { Concentration, } \\
\%\end{array}$ \\
\hline \multirow{2}{*}{250} & Beads & 1.57 & 2.70 & 90 \\
\cline { 2 - 5 } & Water & 0.04 & 0.06 & 2 \\
\hline \multirow{2}{*}{500} & Beads & 1.42 & 1.16 & 2 \\
\cline { 2 - 5 } & Water & 0.02 & 0.06 & 2 \\
\hline
\end{tabular}

Table 8: Summary of measurements of mixing between a Polysciences $1 \mu \mathrm{m}$ beads dispersion $(3 \mu \mathrm{L} / \mathrm{mL})$ and water in a microfluidic device. Measurements were obtained by UV-Vis spectroscopy.

\subsubsection{Mechanical strength}

It is important that a microfluidic device is able to withstand any wear that is likely to be incurred over its intended lifetime. At the very least, device failure should be avoided, but ideally flow properties should also be preserved. Such wear could be expected from two sources:

- Firstly, general handling and transport can place considerable stress on a device. Installation and removal of fittings in particular will place strain on the interconnectors, which could compromise the integrity of the seal if it is not sufficiently strong and rigid. The inherent portability of Lab-on-a-chip devices makes robustness important, as devices may need to endure handling in applications outside a laboratory.

- Secondly, producing fluid flow can create substantial pressure within the device. This is minimal in cases where electro-kinetic and other flow types are employed. However, it is very common to use pressure-driven flow, which is based on creating a pressure gradient within the channel. Therefore devices using this form of flow control must be especially resistant to desealing.

The microfluidic devices fabricated in this project were made by bonding PDMS to PDMS. The resulting devices were fairly soft and pliable compared to PDMS-on-glass devices. Sealing by corona discharge was found to produce a bond between two PDMS surfaces far more reliably than between PDMS and glass. Glass-based devices are also vulnerable to fracture, which is avoided in our design. However, if the device was too thin, it could bend enough to weaken or break the conformal seal between the luer interconnectors and the PDMS. Stress placed on individual connectors when attaching 
and reattaching tubing was also found to unseat the interconnectors in some cases. However, both issues were greatly improved by increasing the thickness of the top PDMS layer. This increased the stiffness of the devices and reduced bending, as well as preventing the connectors from moving.

In order to estimate the ability of the devices to withstand applied pressures, a small number of devices were subjected to destructive testing. For each test, a nitrogen gas cylinder was connected to one of the device inlets via a pressure regulator. The remaining connectors on the device were sealed using cyanoacrylate adhesive. The pressure was then increased in steps of $50 \mathrm{kPa}$ until a leak was formed. Increasing the pressure in the same manner for each device acted to exclude any fatigue effects on the results. At each step the pressure was held for a period of a few minutes. It was found that devices produced using earlier fabrication methods, in which the thickness of the PDMS was minimal, tended to fail at pressures of 50-100 kPa. A leak would invariably form at the interface between the luer and the PDMS surrounding it. This is thought to occur because the compressible PDMS is easily pushed away from the connector by the pressurised gas.

When more recent versions of the device were subjected to pressure testing, the results were greatly improved. Pressures of $200-250 \mathrm{kPa}$ were reached, and failure only occurred several minutes after the pressure was first applied. This suggests that the devices could withstand larger pressures, provided they occur in short pulses. Failure consistently occurred by delamination of the two PDMS layers, rather than at the luerPDMS interface. As discussed in Section 3.1.5, the bond formed between the PDMS layers due to plasma oxidation should resist pressures of $210-375 \mathrm{kPa}$, so this failure is not unexpected. The results make it clear that this revision in the device design has greatly strengthened the fluid connectors, as they are no longer the limiting factor in device strength.

It was estimated in Section 3.3.2 that the pressure required to maintain a flow rate as high as $500 \mu \mathrm{L} / \mathrm{min}$ (of water) would create a pressure difference of $3.6 \mathrm{kPa}$, meaning that all devices tested here would be capable of sustaining such a flow, provided they were handled carefully to avoid damage. The improvements made to the design may 
however prove useful if very high flow rates are needed, or if highly viscous solutions are to be used. In any case, the final device design was considerably more robust than earlier versions.

\subsubsection{Solvent compatibility}

The use of PDMS as a structural material created the risk of unwanted interaction of the device with organic solvents. PDMS is hydrophobic, and many non-polar organic solvents are soluble in the bulk polymer [151]. A comprehensive study of commonly used organic solvents, conducted by Lee et al. [164], found that PDMS will swell by as much as $110 \%$ in certain solvents. The PDMS will then recover to its initial state once the solvent has evaporated. In the case where the solvent is driven through a PDMS microchannel, we would expect some deformation of the structure to occur, due to inhomogeneous exposure of the polymer to the solvent. On account of this effect, the use of organic solvents in PDMS-based devices is typically avoided. Nonetheless, cases of non-polar solvents such as benzene and toluene being used in such devices without damage have been reported [164,202].

An initial goal of this project was to purify a solution of magnetic nanoparticles obtained through the thermal decomposition reaction discussed in Section 3.2. This would avoid the need to purify the reaction product manually. However, the reaction must be carried out in organic solvents (toluene or mesitylene) to avoid unwanted oxidation of the particles. Toluene induces swelling of 30\% in PDMS [164], and as mesitylene is chemically similar to toluene we would expect a similar level of swelling from this solvent.

While a continuous flow of water could be maintained indefinitely, toluene flow could only be carried out briefly before one or more of the connections would rupture. Initially it was suspected that the failure was due to degradation of either the PVC luer adaptors or the silicone solution tubing. Both products are rated as susceptible to damage in toluene by the manufacturer [203]. In practice the damage to the luers was minimal, with some discolouration and softening of the plastic apparent after long periods of use. Samples of the silicone tubing rapidly discoloured when immersed in toluene, and lost some elasticity. Transferring these samples into ethanol led to the precipitation of plastic 
fibres, suggesting that the tubing plastic is soluble in toluene. Although the plastic should remain dissolved, any precipitation could lead to clogging of the microchannels. This would then cause device failure, due to pressure build-up.

In order to exclude the contributions of the tubing and interconnectors, trials were performed using Teflon tubing and nylon luer interconnectors. Both products are resistant to solvent damage [203]. However, even when these alterations were made to the experimental setup, device failure still rapidly occurred. This implied that the solvent incompatibility is related to swelling of the PDMS, rather than damage to the connectors and tubing. This was confirmed by experiments in which these components were excluded entirely. A PDMS microfluidic device was produced without embedded connectors. A flow of toluene was then manually injected using a needle-tipped syringe. Needle tips were used to open the device outlets to atmosphere. It was found that within a short period, substantial resistance to the applied pressure was felt in the syringe. A leak would occur at the injection site if further pressure was applied.

Figure 75 shows the central channel of two identically-prepared PDMS microfluidic devices. In the left-hand image, distilled water was flowed continuously through the channel. The channel walls are clearly visible, with a black colour. The channel was imaged using a backlit microscope, so the dark colour of the walls can be attributed to reduced light transmission in these regions. In the right-hand image, toluene was flowed through the channel. The most striking difference between the images is that the channel walls were much lighter, and also around 70\% wider, when toluene was used. The channel itself was reduced in width by approximately $13 \%$. A prominent ridge was observed on the underside of the device, along the length of the channel, due to swelling of the PDMS. This ridge would disappear once the toluene flow was stopped, as the solvent was allowed to evaporate out of the polymer. While the width reduction alone would not be expected to greatly increase the pressure inside the channel, the image in Figure 75 is consistent with a reduction in the channel height. Because the channel height is around half the channel width, this could lead to a much greater increase in the pressure required to maintain the flow rate. Attempts to directly measure the channel height before and after exposure were inconclusive. 

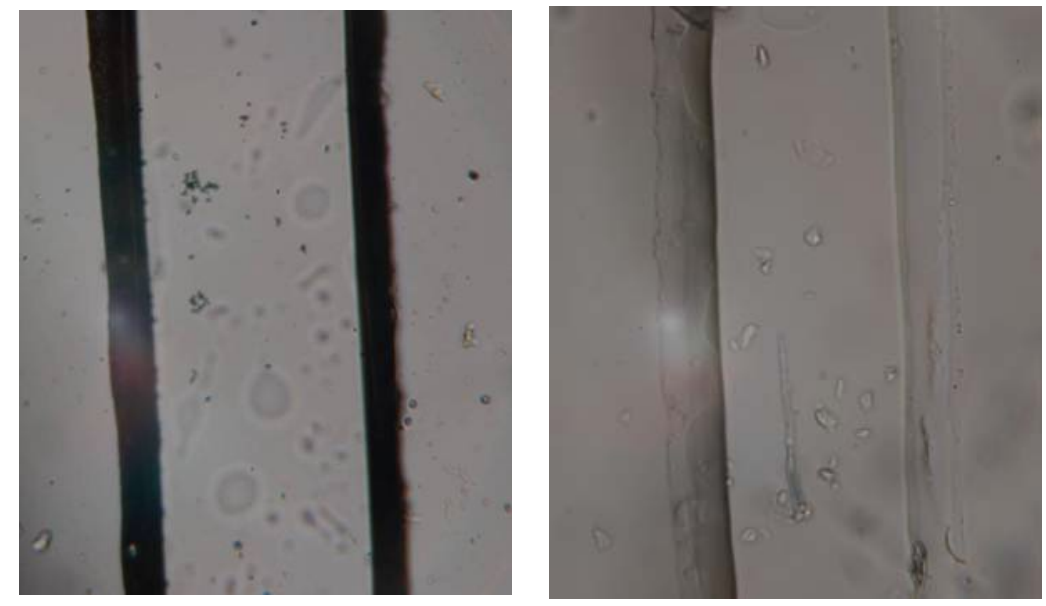

Figure 75: Comparison of a PDMS microchannel filled with distilled water (left) or toluene (right). Swelling of the PDMS by the toluene causes channel constriction.

\subsubsection{Embedded magnet system}

The simplest microfluidic magnetic separation device constructed for this project is pictured in Figure 76. A $4 \times 3$ grid of miniature $\mathrm{NdFeB}$ magnets is embedded in the PDMS, adjacent to the central channel. This configuration was used to prevent the magnets from moving on the substrate during fabrication, but also served to increase the length of the channel exposed to the field. A microscope image of the device is displayed in Figure 77, and shows the spacing between the magnets and the channel wall is approximately $335 \mu \mathrm{m}$, which is comparable to the channel width of $330 \mu \mathrm{m}$.

It was found that the small spacing between the magnets and the channel did not compromise the bond between the PDMS layers, provided that the formation of bubbles was avoided. If bubbles were present in the uncured PDMS, they would tend to get stuck between the magnets and the features on the SU-8 master. Once the PDMS had cured the bubbles would cause leaks to form. This could be avoided by using a needle to either burst bubbles or drag them away from the channel, before allowing the PDMS to cure. 

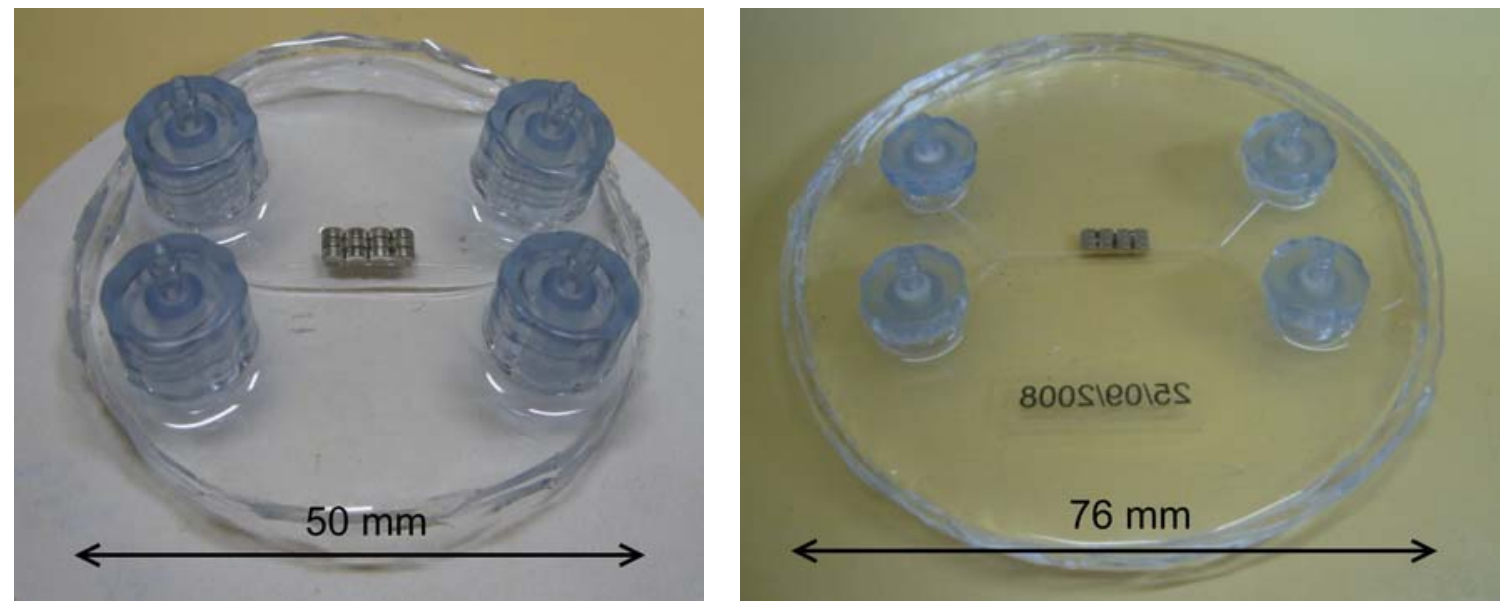

Figure 76: Microfluidic devices with embedded permanent magnets for magnetic separation. Devices made using 2" (left) and 3" (right) PMMA wafers.

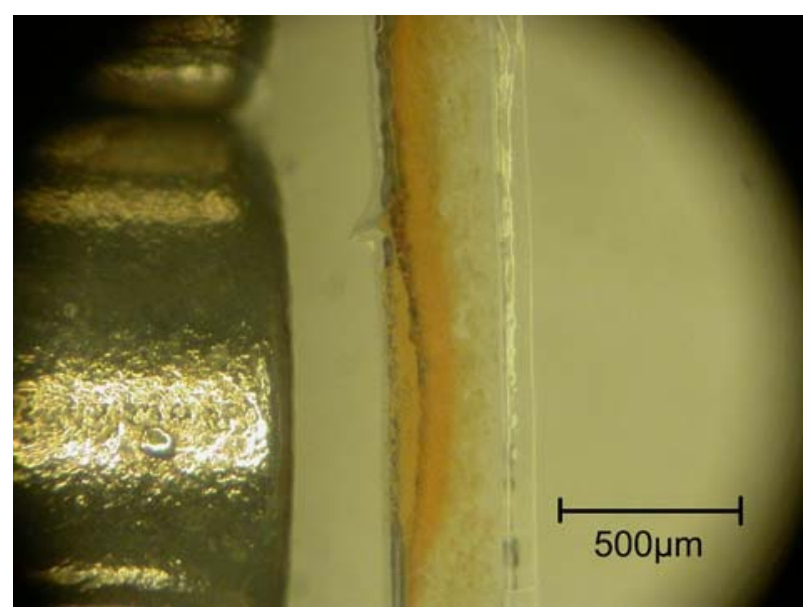

Figure 77: Close-up of the microchannel in the embedded magnet device, showing the proximity of the magnets to the channel wall. Residual particles in the channel are Dynabeads trapped by the magnetic field.

It was not practical to directly measure the effective field gradient inside the channel, due to the very small length scales involved. The expected field gradient was instead calculated using the freeware computer program Finite Element Method Magnetics [6]. A magnetic particle flowing down the length of the central microchannel of this device will move past the surface of the magnet grid. Figure 78 shows the strength of the magnetic field acting on this particle as a function of position down the length of the channel. The magnitude of the field varies between positive and negative, because the adjacent columns of magnets are oriented in opposing directions. The columns of magnets were oriented this way to prevent repulsive forces breaking the array apart. 
Changes in the polarity of the field experienced by superparamagnetic particles should fortunately not have any effect on the separation efficiency, as their internal magnetisation should immediately reverse to align with it. This means that the particles should always experience an attractive force despite the change in field.
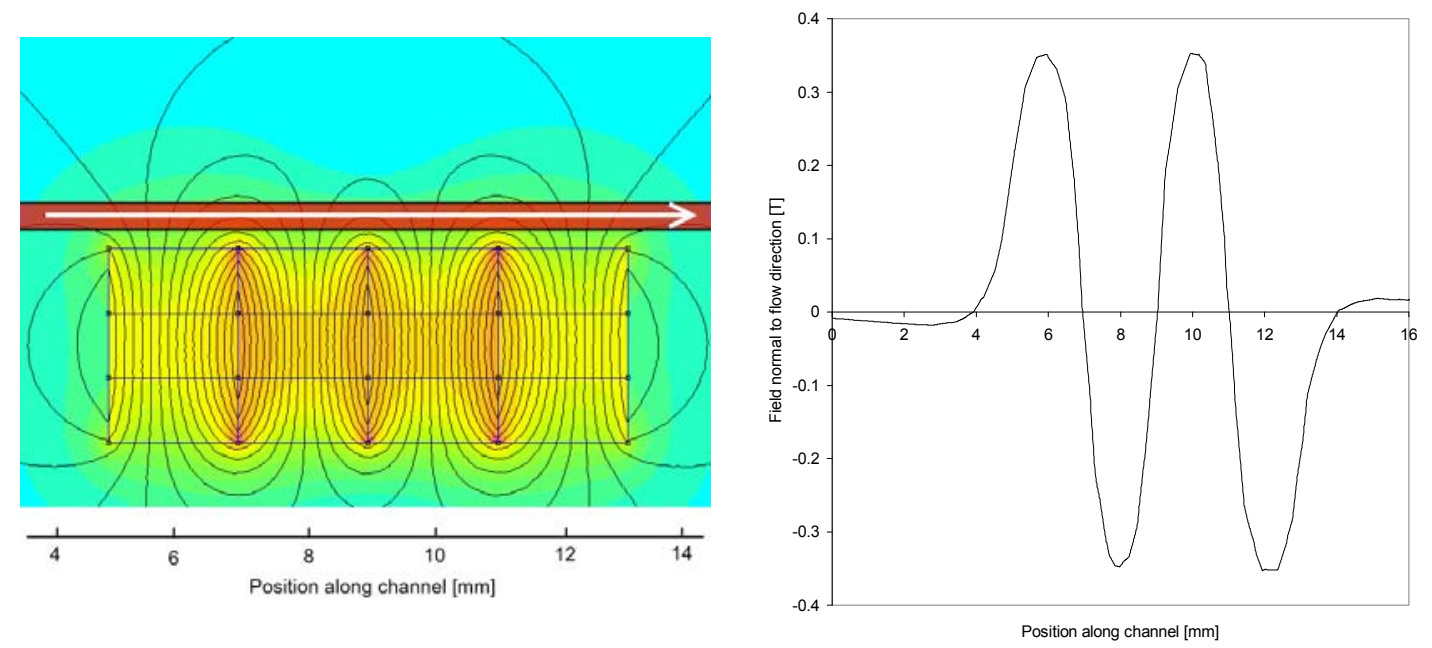

Figure 78: (Left) FEMM diagram showing the magnet grid, and the path taken by a particle flowing along the centre of the main channel (indicated by the arrow). (Right) Magnetic field perpendicular to the channel length, experienced by particle following the specified path.
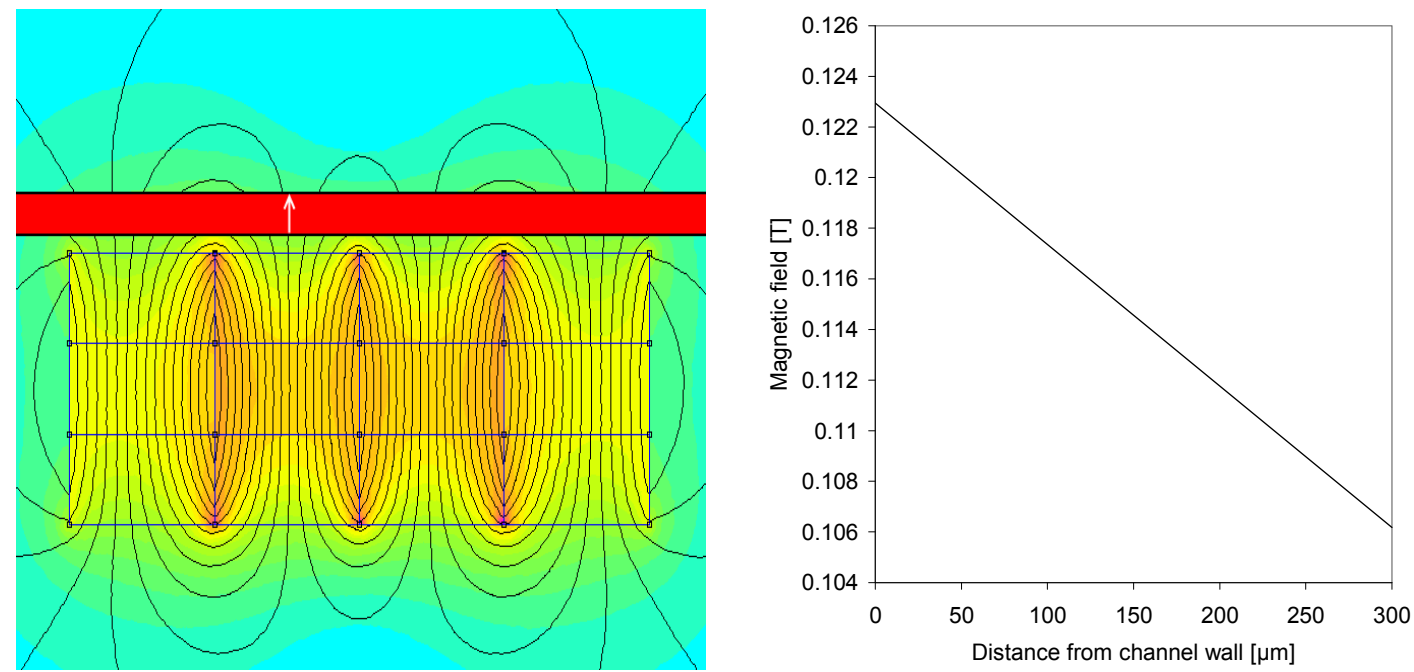

Figure 79: (Left) FEMM diagram showing the magnet grid, and the path taken by a particle moving away from the adjacent channel wall. (Right) Magnetic field experienced by a particle following this path.

In order to quantify the magnetic field gradient for this device design, the magnetic field was plotted as a function of lateral position in the fluid channel. As can be seen in Figure 79 , the field felt by a particle decreases linearly as it moves away from the channel wall closest to the magnets. From the plot an approximate field gradient of $500 \mathrm{~T} / \mathrm{m}$ was 
measured. This is a large value over the length scale $(\sim 0.5 \mathrm{~mm})$ in question. The gradient can be attributed to the alternating polarity of the magnets, which increases the flux density close to the magnet surfaces. In initial simulations the magnets were in a parallel rather than antiparallel configuration, which gave a maximum field gradient of $40 \mathrm{~T} / \mathrm{m}$.

Although this device displayed the largest effective field gradient of any type designed in this project, formal magnetic separation experiments were not performed. This was primarily because the magnets were permanently set into the PDMS, preventing the field gradient being removed when needed. This limitation can lead to particles becoming trapped in the channel, making it difficult to reuse the device.

\subsubsection{Magnet-micrometer system}

As discussed in the previous section, the embedded magnet system produced large field gradients, but did not feature the ability to temporarily deactivate the field. This ability was gained by positioning permanent magnets outside the device. The magnets could not be brought as close to the channel as when they were embedded, meaning the maximum field gradient was reduced, but the magnets could now be readily moved.

From the microscope images presented in Figure 80, the spacing between the notch and the fluid channel is roughly $1.3 \mathrm{~mm}$. The magnet is attached $1 \mathrm{~mm}$ from the end of the metal arm, allowing the magnet to be brought within $2.3 \mathrm{~mm}$ of the channel. Figure 81 shows the magnitude of the magnetic field perpendicular to the channel length, for a particle flowing down the centre of the channel. Here it has been assumed that the magnet is at the closest position, giving the largest field. Unsurprisingly, there is only one peak in the applied field, which occurs as the particle passes the middle of the magnet. 

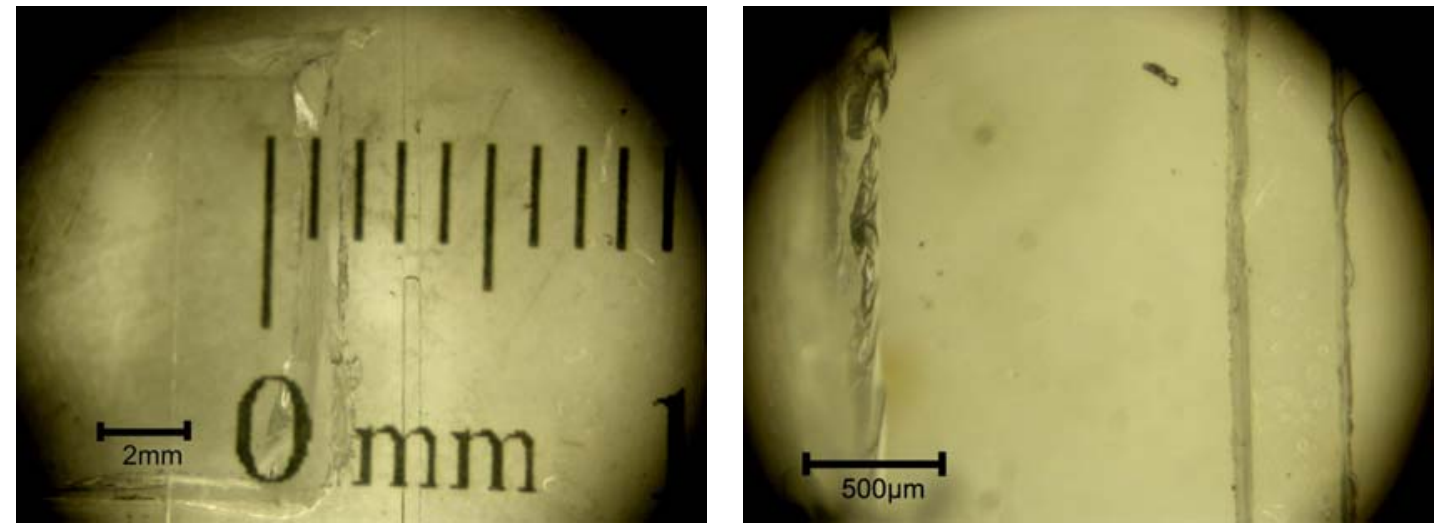

Figure 80: Microscope image showing the main microfluidic channel, and the edge of the notch cut into the PDMS. The separation of these features is about $1300 \mu \mathrm{m}$.
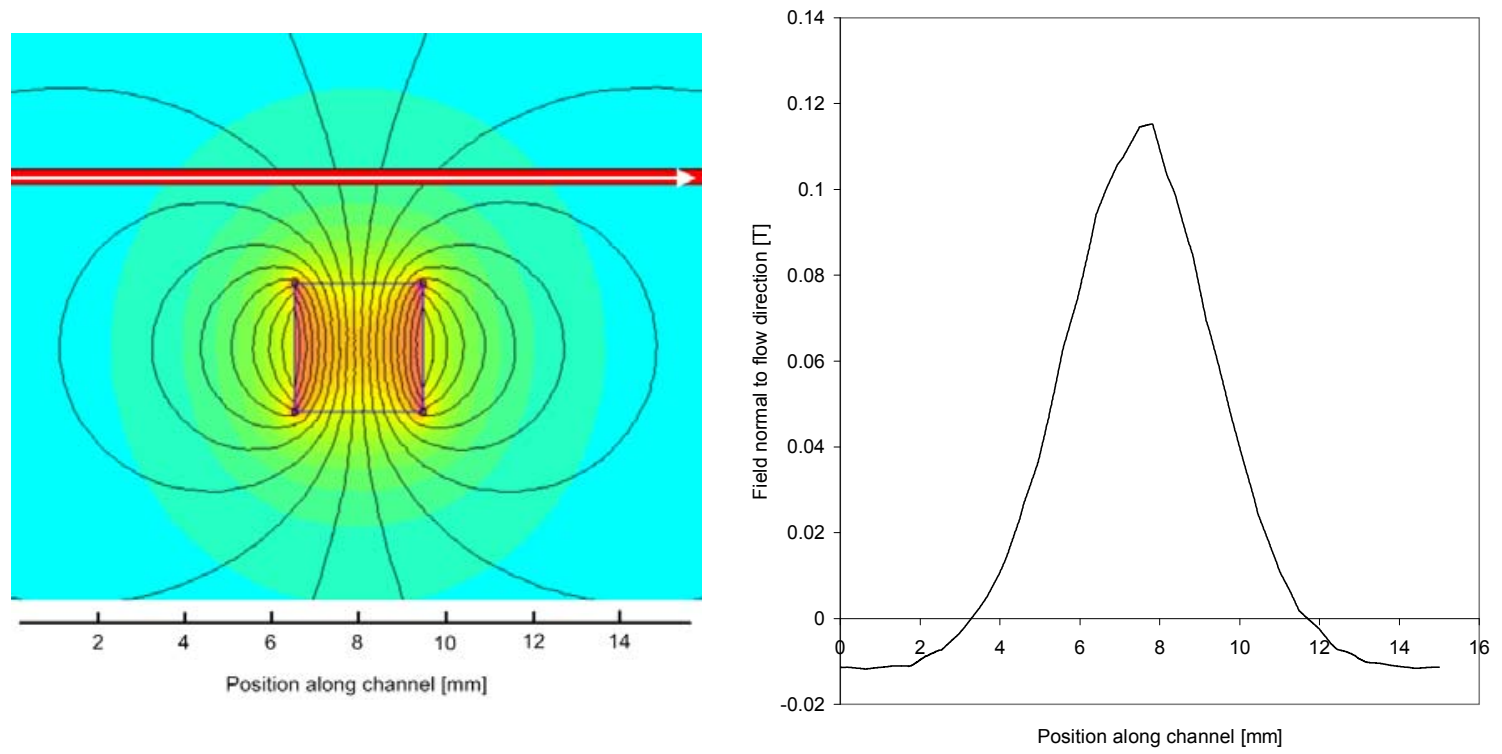

Figure 81: (Left) FEMM diagram showing the magnet geometry, and the path taken by a particle flowing down the centre of the main channel. (Right) Magnetic field perpendicular to the channel, for a particle following the specified path.

It is shown in Figure 82 that the field decreases linearly for a particle moving across the width of the channel, similar to the embedded magnet system. This means the field gradient is constant across the channel, which should help to minimise dispersion in the velocities of particles during magnetic separation. Data from FEMM was used to estimate the average field gradient in the channel, as the magnet is moved away from its closest position. The results are displayed in Figure 83, and show that the gradient may be effectively reduced to zero by adjusting the micrometer dial appropriately, without needing to move the device across the stage. A maximum field gradient of $60 \mathrm{~T} / \mathrm{m}$ can be achieved, which is much less than that achieved using the embedded magnet system, 
but is still large enough for practical use. Larger field gradients could be gained by using more sophisticated methods to cut the PDMS, so that the gap between the notch and channel could be reduced.
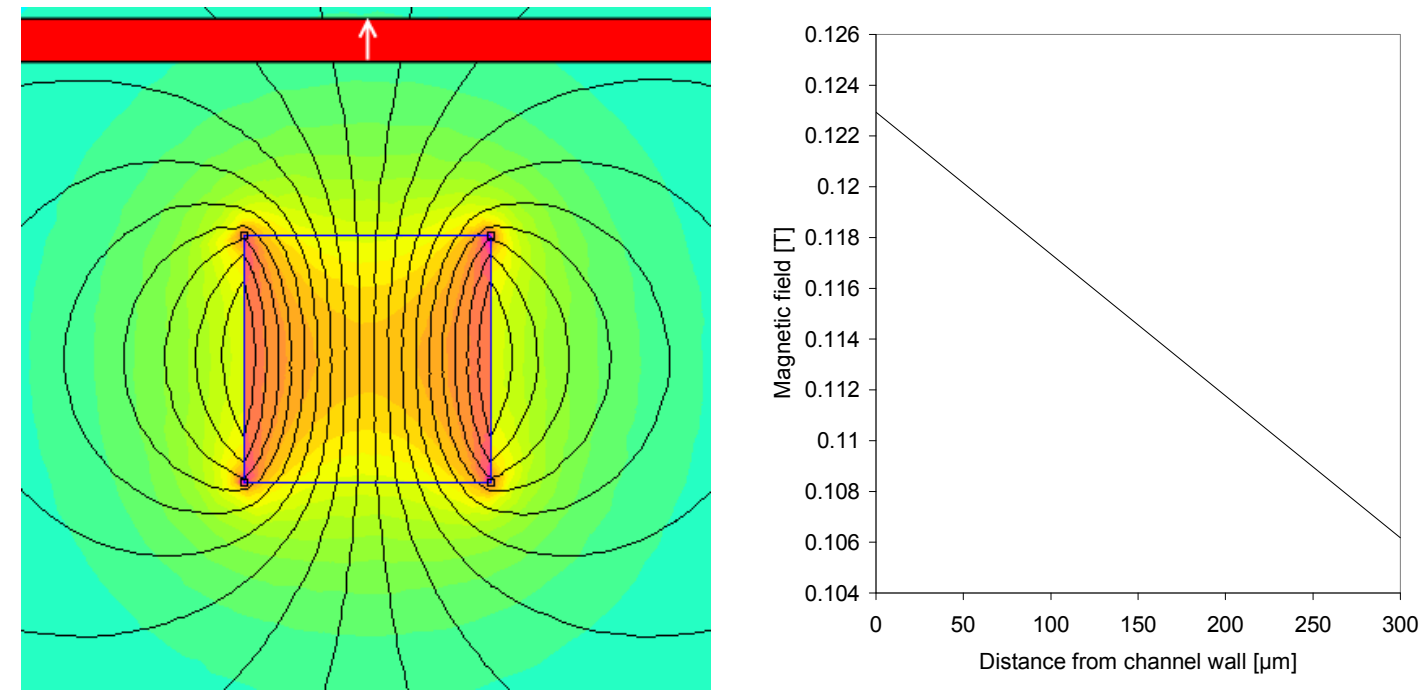

Figure 82: (Left) FEMM diagram showing the magnet geometry, and the path taken by a particle moving across the width of the main channel. (Right) Magnetic field perpendicular to the channel, for a particle following the specified path.

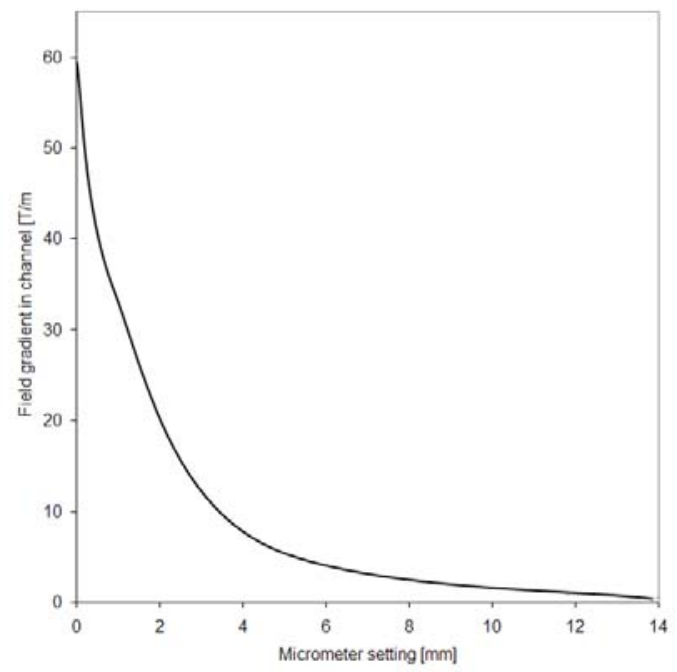

Figure 83: Estimate of the field gradient produced in the channel by the magnet-micrometer system as a function of micrometer setting. A setting of zero corresponded to a channel-magnet spacing of $2.3 \mathrm{~mm}$.

\subsubsection{Metglas system}

Examples in the literature of microfluidic magnetic separation using soft magnetic materials inspired the development of a third device design. The design made use of a commercially available amorphous metal called Metglas, which is supplied in ribbons 
$30 \mu \mathrm{m}$ thick [177]. This avoided the need for expensive fabrication techniques such as metal vapour deposition or electroplating to produce thin metal films.

The most recent version of the Metglas system is pictured in Figure 84. Five strips of Metglas, each $15 \mathrm{~mm}$ long and $2.5 \mathrm{~mm}$ wide, are embedded between the layers of PDMS. Recesses were defined in the master to contain the strips, and allow for better sealing between the PDMS layers. From the microscope image in the same figure, we can see that these recesses are positioned about $200 \mu \mathrm{m}$ from the channel. This gap is much smaller than the $300 \mu \mathrm{m}$ spacing defined in the photomask. As discussed in Section 4.3.1, the incoherent UV-source increases the width of features in the master. It follows that the spacing between adjacent features will also be reduced. The gap was sufficient to prevent fluid leaking from the channel into the recesses, although leaks would often occur if a smaller gap was used.
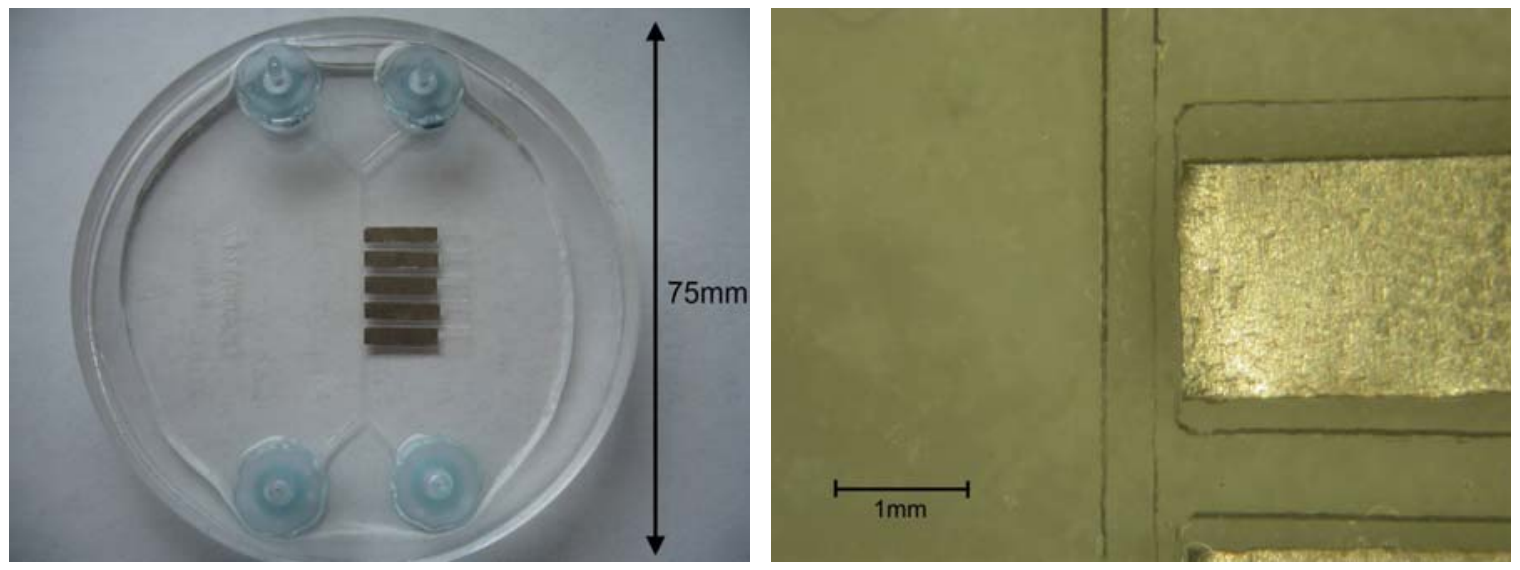

Figure 84: (Left) Microfluidic device with embedded Metglas strips for magnetic separation. (Right) Close-up view of the main channel and one of the 5 Metglas strips. The channel width is $375 \mu \mathrm{m}$.

Numerical simulations in FEMM were used to predict the field gradients generated by a single Metglas strip. The Metglas was magnetised using a $25 \mathrm{~mm}$ diameter NdFeB magnet (surface flux density $\sim 500 \mathrm{mT}$ ), which was placed approximately $8 \mathrm{~mm}$ above the strip. The results are shown in Figure 85, and predict a gradient of $25 \mathrm{~T} / \mathrm{m}$ at the midpoint of the channel, which increases to $60 \mathrm{~T} / \mathrm{m}$ at the channel wall. The values were limited primarily by the size of the gap between the Metglas and the channel, as field gradients of several hundred $\mathrm{T} / \mathrm{m}$ can be reached at distances of less than $100 \mu \mathrm{m}$. This compares to a field gradient of $6 \mathrm{~T} / \mathrm{m}$ produced by the magnet alone. 


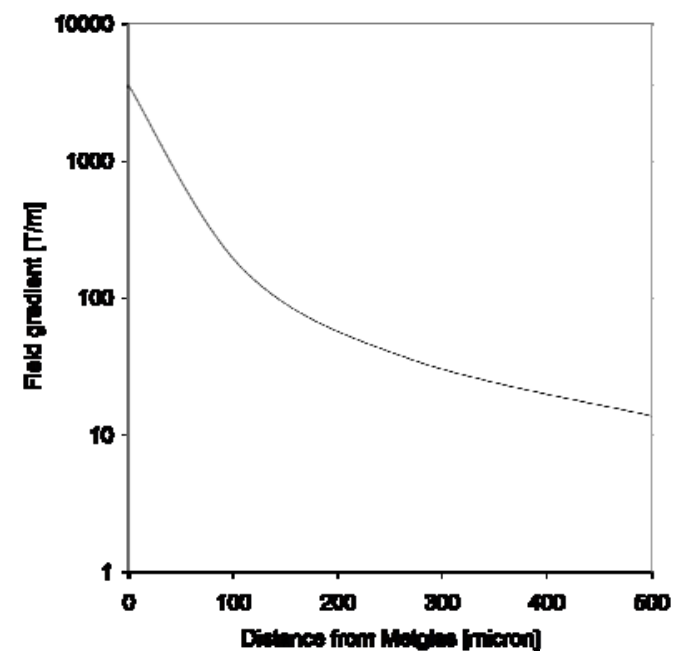

Figure 85: Estimate of the magnetic field gradient produced within the channel by the Metglas system as a function of distance from the tips of an isolated Metglas strip. Field gradient is plotted on a logarithmic scale.

\subsection{Magnetic separation}

In this section, key results from separation experiments using a PDMS-based microfluidic device will be presented. The majority of the experiments were conducted using the magnet-micrometer system, which had shown the best repeatability. Separations were generally performed using Dynabeads MyOne microbeads, due to their availability and good resistance to sedimentation.

The main focus of the experiments was to divert magnetic particles between solutions as efficiently as possible. The ability to select superparamagnetic particles over diamagnetic particles was also investigated, as was the ability to select one size of superparamagnetic bead over another.

The separation efficiency of the devices was usually determined qualitatively through direct imaging, and quantitatively through spectroscopy. In a few cases where spectroscopy was not practical, direct mass measurement was employed. Size selectivity was determined through particle counting from microscope images, as spectroscopy could not be used to distinguish between particle sizes. A more detailed discussion of the observation techniques was previously provided in Section 3.6.3. 


\subsubsection{Control}

When determining the separation efficiency of the device, it is essential to take into account the proportion of particles that would have been transferred to the buffer stream, without a magnetic force present. If this proportion is high, the selectivity of the device will be compromised, as particles that should have been excluded on the basis of their magnetic properties may be collected. The selectivity may be affected by a number of factors. Firstly, disruptions in the fluid flow may force some particles through the wrong outlet. Secondly, particles may diffuse into the buffer stream. As discussed in Section 2.3.3, this effect theoretically should be negligible for micron-sized beads, but becomes significant for nanoparticles. Diffusion also becomes more important at lower flow rates, as particles have more time to diffuse across the boundary. Finally, blockages by bubbles may occur, although the application of negative pressure at the outlets is expected to quickly remove them.

The degree of non-selective separation occurring in the magnet-micrometer system was measured. This involved running a solution of Dynabeads through the magnetic separation setup in the absence of a magnetic field. The experiment was carried out at a flow rate of $5 \mu \mathrm{L} / \mathrm{min}$, as this was the slowest flow rate used for quantitative measurements. We would expect the factors acting to reduce the selectivity (particularly diffusion) to be most severe at low flow rates. The results of this control experiment are summarised in Table 9.

\begin{tabular}{|c|c|c|}
\hline Fraction & Concentration (beads $/ \mathrm{mL})$ & \% of original solution \\
\hline Original & $6.54 \times 10^{8}$ & 100 \\
\hline Inlet & $1.83 \times 10^{8}$ & 28.0 \\
\hline$S 1$ & $1.74 \times 10^{7}$ & 2.7 \\
\hline$S 2$ & $5.16 \times 10^{7}$ & 7.9 \\
\hline$U 1$ & $1.23 \times 10^{8}$ & 18.8 \\
\hline$U 2$ & $1.36 \times 10^{8}$ & 20.9 \\
\hline
\end{tabular}

Table 9: Concentrations of fractions collected after flowing a solution of Dynabeads through a microfluidic device at $5 \mu \mathrm{L} /$ min with no magnetic field present. 
Almost a third of the beads settled in the tubing before even entering the device. The number of particles which settled in this fashion was found to vary significantly between experiments, possibly due to variations in the lengths of tubing used. The beads are too large for Brownian motion to prevent them from settling, although as discussed in Section 2.4.4 it should reduce the settling rate. We can estimate the upper limit of the settling velocity by considering the balance between the gravitational and fluid drag forces, in the absence of thermal motion. Therefore, setting Equations (20) and (21) equal gives

$$
u=\frac{2}{9} \Delta \rho g r^{2}
$$

where $\Delta \rho$ is the relative density of the bead $\left(0.8 \mathrm{~g} / \mathrm{cm}^{3}\right), r$ is the particle radius and $g$ is the gravitational force. For a Dynabead the settling velocity is just $4.5 \times 10^{-10} \mathrm{~m} / \mathrm{s}$, meaning gravity should move a bead no more than $3 \mu \mathrm{m}$ over the course of the experiment. This is much smaller than the tubing diameter of $2 \mathrm{~mm}$, so we would not predict the substantial amount of settling which was observed in practice. However, it is possible that some beads may form into clusters, which would accelerate sedimentation.

About $10 \%$ of the particles were collected in the buffer stream. It was previously shown in Figure 14 that at $5 \mu \mathrm{L} / \mathrm{min}, 1 \mu \mathrm{m}$ particles will diffuse up to $8 \mu \mathrm{m}$ into the buffer stream. Integrating over the concentration gradient leads to the prediction that just $0.5 \%$ of the particles should cross the flow boundary due to diffusion alone. Therefore there must be other contributions to the observed result. Because negative pressure was used to maintain the flow rate at the outlets, bubbles or defects in the channel geometry should not be significant. It is likely that pressure variations reduced the steadiness of the laminar flow boundary, and allowed some particles to exit at the wrong outlet. It will also be shown in Section 4.4.2 (see Figure 87) that there is some evidence of localised turbulence near the outlet junction, which may cause particle transfer.

It would be possible to combat the above effect by flowing the buffer at a faster rate than the particle solution. This would in turn increase the width of the buffer stream in the channel, so that some of the buffer exits at the opposing outlet. The particles would then need to travel further to exit at the collection outlet, making unintentional transfer less 
likely. However it would be necessary to use both pumps to independently control the flow at the inlets, leaving the outlets open to atmosphere. Blockages by bubbles may then become a problem, unless extra pumps could be obtained for withdrawing the fluid.

A further $34 \%$ of the particles failed to exit at one of the outlets, instead settling at the bottom of the luer connectors. Particles did not appear to settle in the channel itself, indicating that the large dead volume of the luers was the main problem. The volumetric flow rate $\mathrm{Q}$ is equal at all points in the device. This means that the flow velocity is smaller in areas with a larger cross-sectional area. The channel is approximately rectangular with dimensions of $150 \times 330 \mu \mathrm{m}$, giving a cross-sectional area of $5.0 \times 10^{-8}$ $\mathrm{m}^{2}$. The base of a luer adaptor has an inner diameter of $4 \mathrm{~mm}$, presenting a cross-section of $1.3 \times 10^{-5} \mathrm{~m}^{2}$. The cross-section of the interconnects is approximately 1000 times larger than that of the channel, meaning the fluid will flow around 1000 times slower. The main diameter of the luer is much greater than both the channel reservoir and the outlet barb, which may lead to a non-uniform flow velocity inside the luer.

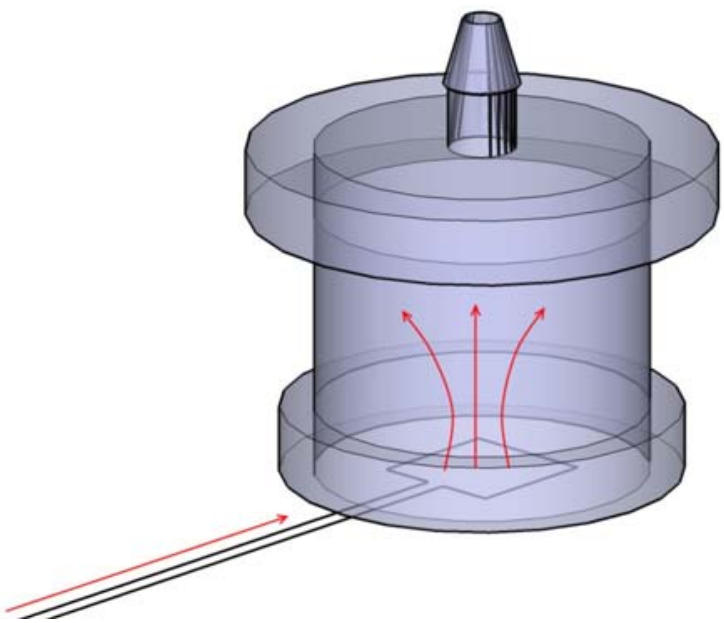

Figure 86: Schematic of a luer adaptor connected to a microchannel fluid reservoir. The large internal volume of the adaptor, combined with abrupt changes in the cross-section, may trap particles inside the luer.

Finally, it is worth noting that the fraction concentrations only sum to about $80 \%$ of the original solution concentration, implying that some particles remained uncollected. The most significant losses were thought to occur through the settling of particles in the base of the inlet luer, and the possible trapping of particles against the sidewall of the fluid channel next to the magnet. In addition, water left behind after cleaning the microfluidic 
device and tubing would be expected to dilute the solution. This would reduce the apparent concentration of the fractions collected. This effect was previously observed in experiments using a food dye solution (see Section 4.3.2), and although it was reduced by syringing out most of the water after cleaning, it was not eliminated entirely. Flowing air through the channels prior to an experiment could be used to achieve more thorough drying if necessary.

\subsubsection{Basic separation}

The simplest magnetic separation experiments performed involved diverting magnetic microbeads into a buffer stream as efficiently as possible. These experiments were performed using the magnet-micrometer system, which allowed the effective field gradient to be adjusted by moving the magnet. The effect of the gradient on a solution of Dynabeads $\left(\sim 6 \times 10^{9}\right.$ beads $\left./ \mathrm{mL}\right)$ was first investigated visually. As discussed in Section 3.6.1, a concentration this large was required in order to allow visual observation using a microscope.

As shown in Figure 87, the dark microparticle dispersion did not mix with the buffer as the two flows entered the channel. When the field gradient was small, the streams remained distinct and exited at their respective outlets. Only a minimal quantity of particles was transferred into the buffer stream. Increasing the field gradient caused more of the particles to be drawn across the laminar flow boundary. By the time the maximum field gradient of $60 \mathrm{~T} / \mathrm{m}$ was reached, more particles exited in the buffer than remained in the original solution.

It can be seen in Figure 87 that a small cloud of particles was present just upstream of the outlet junction of the channel. This cloud appeared to originate at the interface between the buffer and solution streams, and extended across the width of the buffer stream. The shape and size of the plume was similar for all values of the field gradient, suggesting that these particles were not being drawn across the channel by the magnetic field. It is possible that localised turbulent flow was generated in this region, which may drive some particles across the channel and into the buffer stream. As discussed in Section 4.3.2, turbulence in particle-laden flows is less readily damped by friction against the walls. The particle cloud was only observed when the concentration was 
high, although it is difficult to determine if the cloud was simply too faint to see at lower concentrations.

An increasing number of particles became trapped against the channel wall adjacent to the magnet, which is an undesired side-effect of the separation process. This occurred due to the formation of clusters of beads in the channel. These clusters will move towards the magnet faster than single particles. We therefore cannot adjust the field gradient to optimally capture all the particles, unless the concentration is low enough to prevent agglomeration from occurring.

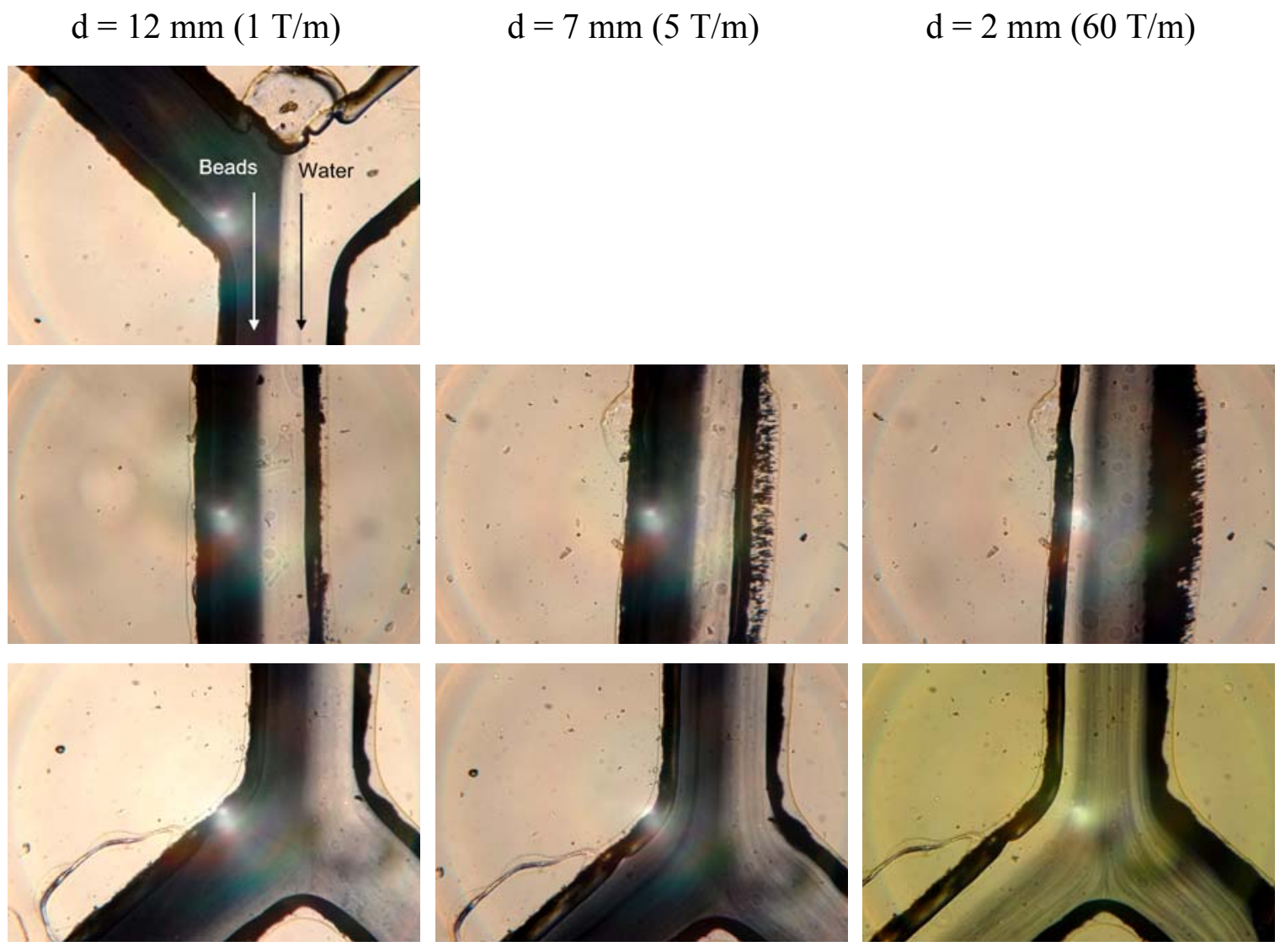

Figure 87: Magnetic separation of an aqueous dispersion of Dynabeads (concentration $\sim 6 \times 10^{8} \mathrm{~mL}^{-1}$ ) at a flow rate of $10 \mu \mathrm{L} / \mathrm{min}$. The distance between the magnet and the channel was changed to vary the field gradient. The process was observed at $1 \mathrm{~T} / \mathrm{m}$ (left), $5 \mathrm{~T} / \mathrm{m}$ (centre) and $60 \mathrm{~T} / \mathrm{m}$ (right). Images were taken at the input junction (top), main channel (centre) and outlet junction (bottom), using a $4 \mathrm{x}$ microscope objective.

The effect of flow rate on the separation process is illustrated in Figure 88, in which the field gradient was kept constant at $60 \mathrm{~T} / \mathrm{m}$. The nominal concentration was unchanged 
from the previous result. However, because the solution was frequently reused for qualitative experiments, the actual concentration was somewhat reduced, and the image contrast is therefore lower. The striking differences from the results in Figure 87, even where the nominal conditions overlap, may be partly caused by this effect. Decreasing the flow rate has a similar effect to increasing the field gradient, as the particles are exposed to the field gradient for a longer time. This increases the likelihood that the particles will be drawn into the buffer stream.

As can be seen in Figure 88, the majority of the particles were collected in the buffer once the flow rate was reduced to $10 \mu \mathrm{L} / \mathrm{min}$, but a large amount of material accumulated at the channel wall. This was caused partly because the flow was briefly paused each time the rate was changed. The high bead concentration used may also have contributed. Experiments conducted for quantitative analysis, which used lower concentrations, showed lesser amounts of accumulation at the walls. Once particles begin to build up on the channel wall, they will alter the field gradient inside the channel. This will cause the magnetic separation efficiency to increase with time, when more consistent separation behaviour is typically preferred. 
$50 \mu \mathrm{L} / \mathrm{min}$
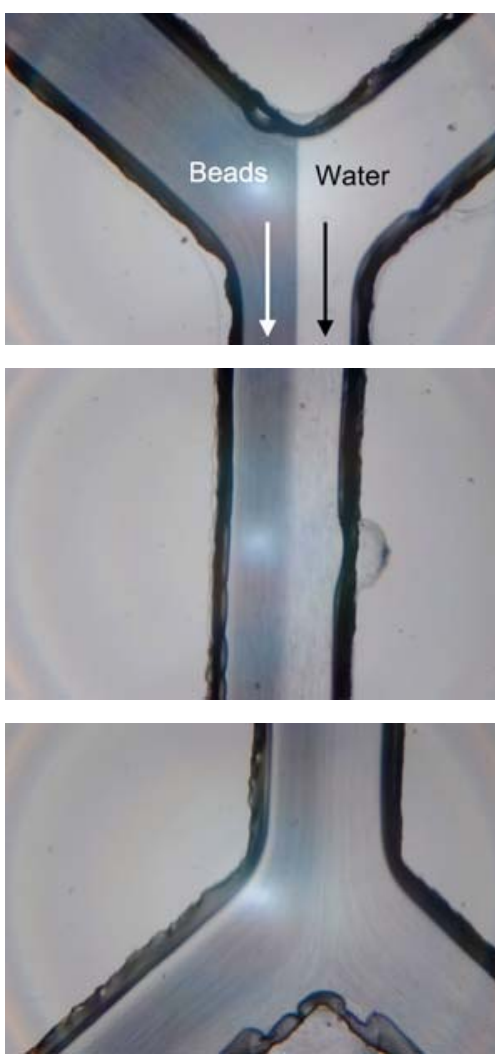

$20 \mu \mathrm{L} / \mathrm{min}$

$10 \mu \mathrm{L} / \mathrm{min}$
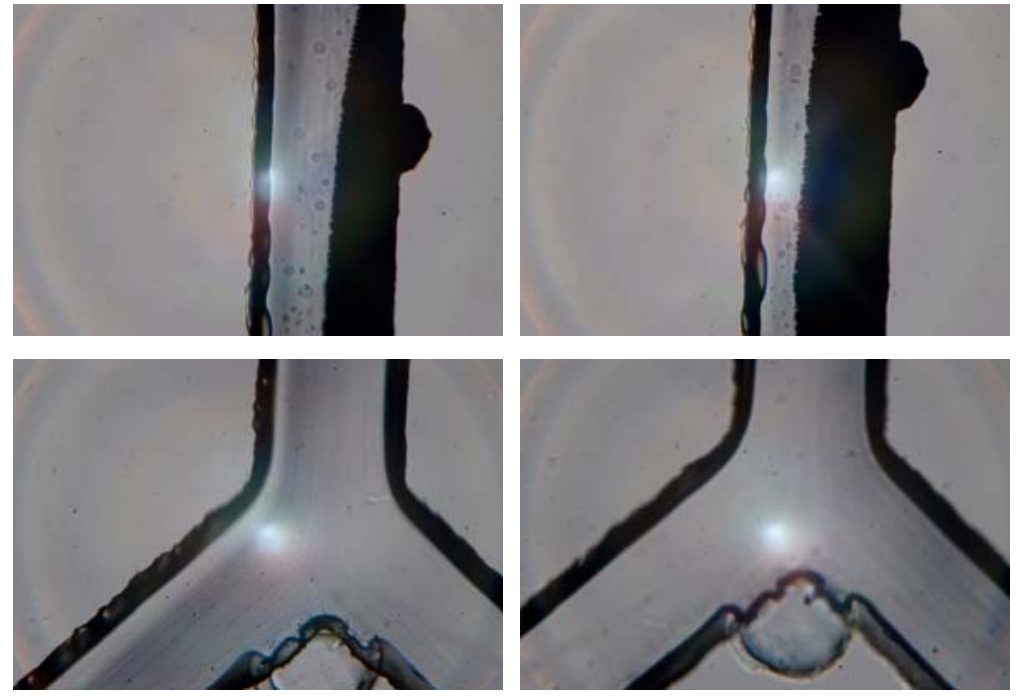

Figure 88: Magnetic separation of an aqueous dispersion of Dynabeads (concentration $\sim 6 \times 10^{8} \mathrm{~mL}^{-1}$ ) in a field gradient of $60 \mathrm{~T} / \mathrm{m}$. The process was observed at $50 \mu \mathrm{L} / \mathrm{min}$ (left), $20 \mu \mathrm{L} / \mathrm{min}$ (centre) and $10 \mu \mathrm{L} / \mathrm{min}$ (right). Images were taken at the input junction (top), main channel (centre) and outlet junction (bottom), using a $4 \mathrm{x}$ microscope objective.

Further experiments were conducted to quantify the magnetic separation efficiency of the device. These experiments consumed larger volumes of solution than qualitative measurements, so the concentration was reduced in order to conserve material. A reduction in concentration will also reduce the amount of particle agglomeration that occurs. Consequently a lower flow rate (or a larger field gradient) is required to maintain the same magnetic separation efficiency. At very low concentrations the beads should act independently, making it possible to use Equation (31) to place a lower limit on the velocity of the Dynabeads:

$$
u_{\text {mag }}=\frac{\left(6.06 \times 10^{-19} \mathrm{~m}^{3}\right)\left(40 \times 10^{3} \mathrm{Am}^{-1}\right)\left(60 \mathrm{Tm}^{-1}\right)}{6 \pi\left(10^{-3} \mathrm{~Pa} \cdot \mathrm{s}\right)\left(0.5 \times 10^{-6} \mathrm{~m}\right)}=150 \mu \mathrm{ms}^{-1} .
$$

The magnetic field gradient will draw the Dynabeads across the channel at a rate of 150 $\mu \mathrm{m} / \mathrm{s}$, meaning it will take an average of 1.1 seconds for the beads to cross into the 
buffer stream. This corresponds to a flow rate of $4 \mu \mathrm{L} / \mathrm{min}$. The particles are unlikely to truly act independently at the concentrations used, so a slightly larger flow rate of 5 $\mu \mathrm{L} / \mathrm{min}$ was used in practice.

Measurements of the magnetic separation efficiency at a flow rate of $5 \mu \mathrm{L} / \mathrm{min}$ are summarised in Table 10. A Dynabeads solution containing $6.5 \times 10^{8}$ beads $/ \mathrm{mL}$ was exposed to a field gradient of $60 \mathrm{~T} / \mathrm{m}$. Again, fractions were not collected from inside the fluid connectors, where many particles settle. As such, less than $20 \%$ of the particles are recovered. However, if the sedimented particles are not considered, a magnetic separation efficiency of $75 \%$ is achieved.

\begin{tabular}{|c|c|c|}
\hline Fraction & Concentration (beads/mL) & \% of original solution \\
\hline$I$ & $3.06 \times 10^{6}$ & 0.8 \\
\hline$S 1$ & $5.23 \times 10^{7}$ & 12.9 \\
\hline$U 1$ & $1.73 \times 10^{7}$ & 4.3 \\
\hline
\end{tabular}

Table 10: Concentrations of fractions collected after magnetic separation of Dynabeads at $5 \mu \mathrm{L} / \mathrm{min}$ in a field gradient of $60 \mathrm{~T} / \mathrm{m}$.

The effect of the field gradient on the separation efficiency was measured by conducting similar experiments at a few different combinations of flow rate and magnetic field gradient. The same bead concentration of $6.5 \times 10^{8}$ beads $/ \mathrm{mL}$ was used in all of the measurements. The results were compared by considering the ratio

$$
r=\frac{S 1}{U 1}
$$

where S1 and U1 represent the concentration of particles in fluid withdrawn from the buffer and solution outlets respectively, as was defined in Section 3.6.3. This led to the results shown in Figure 89. These results clearly show that the magnetic separation efficiency is proportional to the magnetic field gradient, although there is insufficient data to establish the exact nature of the relationship. Based on Equation (31) we would expect the ratio to scale linearly with the field gradient. However, particle agglomeration may be responsible for the non-linear dependence observed, as Equation (31) assumes that the beads act independently. 


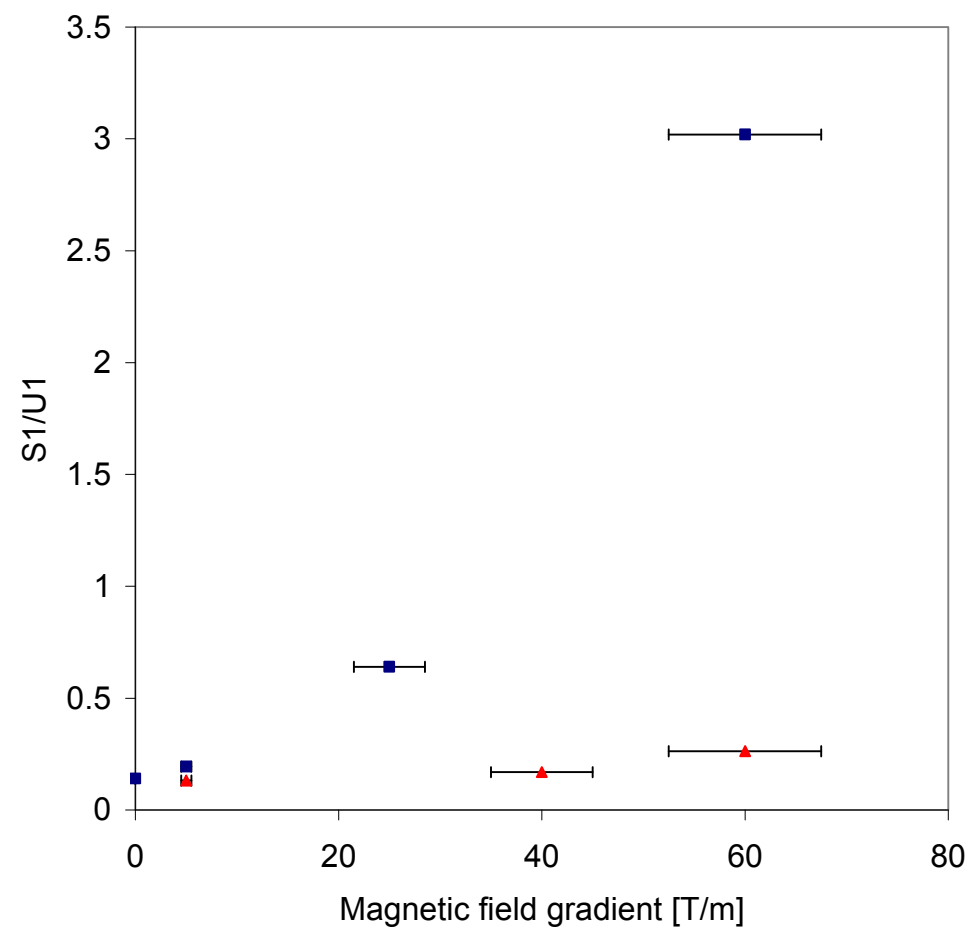

Figure 89: Ratio of separated to unseparated Dynabeads as a function of magnetic field gradient. Data collected at flow rates of $5 \mu \mathrm{L} / \min (\mathbf{a})$ and $25 \mu \mathrm{L} / \min (\Delta)$. The uncertainty in the field gradient was estimated by assuming an uncertainty in the magnet position of $0.5 \mathrm{~mm}$.

\subsubsection{Magnetic moment selection}

In order to evaluate the capability to selectively collect magnetic particles, a run was performed using a solution containing both magnetic and nonmagnetic $1 \mu \mathrm{m}$ polystyrene beads. The solution contained non-magnetic Polybeads at a concentration of $3.48 \times 10^{9}$ beads $/ \mathrm{mL}$, and magnetic Dynabeads at a concentration of $6.56 \times 10^{8}$ beads $/ \mathrm{mL}$. The solution therefore contained roughly five times as many nonmagnetic beads as magnetic beads. The experiment was performed at a flow rate of $6 \mu \mathrm{L} / \mathrm{min}$, in a magnetic field gradient of $60 \mathrm{~T} / \mathrm{m}$.

Concentration measurements for the fractions collected are summarised in Table 11. For each fraction the following ratio was also calculated:

$$
r=\frac{\text { concentration of nonmagnetic beads }}{\text { concentration of magnetic beads }} \text {. }
$$

Overall this ratio was reduced from 5.30 to 2.69 by the separation process. By contrast the ratio was increased to 8.58 on the side opposing the magnet. These results indicate 
that the device showed some selectivity towards magnetic particles. Ideally the ratio would be reduced to below 1, which would correspond to there being more magnetic beads than nonmagnetic beads in the final solution.

\begin{tabular}{|c|c|c|c|}
\hline Fraction & $\begin{array}{c}\text { Polybeads } \\
\text { concentration } \\
\text { (beads } / \mathrm{mL} \text { ) }\end{array}$ & $\begin{array}{c}\text { Dynabeads } \\
\text { concentration } \\
\text { (beads/mL) }\end{array}$ & Ratio \\
\hline Original & $3.48 \times 10^{9}$ & $6.56 \times 10^{8}$ & 5.30 \\
\hline S1 & $6.08 \times 10^{8}$ & $1.20 \times 10^{8}$ & 5.07 \\
\hline$S 2$ & $2.23 \times 10^{8}$ & $1.89 \times 10^{8}$ & 1.18 \\
\hline$S 1+S 2$ & $8.32 \times 10^{8}$ & $3.09 \times 10^{8}$ & 2.69 \\
\hline$U 1$ & $1.67 \times 10^{9}$ & $7.04 \times 10^{7}$ & 23.7 \\
\hline$U 2$ & $2.08 \times 10^{8}$ & $1.48 \times 10^{8}$ & 1.41 \\
\hline$U 1+U 2$ & $1.87 \times 10^{9}$ & $2.18 \times 10^{8}$ & 8.58 \\
\hline
\end{tabular}

Table 11: Concentrations of fractions collected after flowing a mixture of Dynabeads and Polybeads through a microfluidic device at $6 \mu \mathrm{L} / \mathrm{min}$ in a field gradient of $60 \mathrm{~T} / \mathrm{m}$.

It is interesting to note that the ratio of nonmagnetic particles to magnetic ones is greatly inflated for fractions where the particles had settled inside the connectors. This suggests an additional effect occurs, which is selective towards the magnetic particles. A likely explanation is that the larger density of the Dynabeads $\left(1.8 \mathrm{~g} / \mathrm{cm}^{3}\right)$ compared to the Polybeads $\left(1.05 \mathrm{~g} / \mathrm{cm}^{3}\right)$ makes them much more prone to settling inside the connectors. The gravitational settling velocity, as found using Equation (38), is $4.5 \times 10^{-10} \mathrm{~m} / \mathrm{s}$ for the Dynabeads, and $2.8 \times 10^{-11} \mathrm{~m} / \mathrm{s}$ for the Polybeads. The much larger settling velocity of the Dynabeads should make them more likely to settle in the luers than the lighter Polybeads.

Although the magnet used for the separation was positioned at the midpoint of the main fluid channel, a magnetic field will still be present at other points in the channel and the connectors. It could therefore be argued that the Dynabeads settle in the luer connectors 
due to the magnetic field. This could occur either because the field gradient draws beads towards the magnet, or because the magnetised beads are attracted to each other and agglomerate.

The small size of the magnet used meant that the field gradient was no more than 0.5 $\mathrm{T} / \mathrm{m}$ inside the luer adaptors, which is less than one hundredth of the maximum field gradient of $60 \mathrm{~T} / \mathrm{m}$. The magnitude of the magnetic field was also less than $9 \mathrm{mT}$, which will only produce a magnetisation of about $1 \times 10^{4} \mathrm{~A} / \mathrm{m}$ in the beads. This is well below the saturation magnetisation of $4.76 \times 10^{4} \mathrm{~A} / \mathrm{m}$ reported in Section 4.2.2. However, according to Equation (29) it would still take $5.5 \times 10^{-18} \mathrm{~J}$ to break apart two beads in direct contact under these conditions, which is much greater than the available thermal energy. The possibility that particles agglomerate in the luer adaptors due to the magnet therefore cannot be excluded. At the same time, Equation (29) does not predict the rate of agglomeration, so it is not known just how significant an effect it will have.

\subsubsection{Size selection}

As discussed in Section 2.4.6, it should be possible to select magnetic particles on the basis of their magnetophoretic mobility. The ability to achieve this using our device was evaluated by attempting to separate $5 \mu \mathrm{m}$ magnetic beads from $2 \mu \mathrm{m}$ magnetic beads. Both bead types were supplied by Spherotech, and have similar magnetic properties. Using Equation (32) and the magnetisation data presented in Section 4.2.2, we estimate the relative mobility to be

$$
\frac{u_{5 \mu m}}{u_{2 \mu m}}=\left(\frac{5.70 \times 10^{3} \mathrm{~A} / \mathrm{m}}{8.54 \times 10^{3} \mathrm{~A} / \mathrm{m}}\right)\left(\frac{2.5 \times 10^{-6} \mathrm{~m}}{1 \times 10^{-6} \mathrm{~m}}\right)^{2}=4.2,
$$

which suggests that the $5 \mu \mathrm{m}$ beads will move more than four times faster than the $2 \mu \mathrm{m}$ particles in a field gradient. We can then estimate the average velocity induced in the $5 \mu \mathrm{m}$ beads by the magnet-micrometer system using Equation (31)

$$
u_{5 \mu m}=\frac{\left(6.5 \times 10^{-17} \mathrm{~m}^{3}\right)\left(5.7 \times 10^{3} \mathrm{Am}^{-1}\right)\left(60 \mathrm{Tm}^{-1}\right)}{6 \pi\left(10^{-3} \mathrm{~Pa} \cdot \mathrm{s}\right)\left(2.5 \times 10^{-6} \mathrm{~m}\right)}=470 \mu \mathrm{ms}^{-1} .
$$


It would therefore take an average of 0.25 seconds for the $5 \mu \mathrm{m}$ beads to move into the buffer stream. Thus for a $3 \mathrm{~mm}$ wide magnet we want the particles to flow at a linear velocity of $12 \mathrm{~mm} / \mathrm{s}$, corresponding to a flow rate of $18 \mu \mathrm{L} / \mathrm{min}$.

Unfortunately these experiments were conducted before magnetic measurements could be performed, meaning the much larger magnetisation of another Spherotech product was assumed. This prompted the use of a much larger flow rate of $45 \mu \mathrm{L} / \mathrm{min}$, which will greatly reduce the amount of material recovered. Nonetheless, material which is successfully recovered should still contain a larger amount of the $5 \mu \mathrm{m}$ beads.

Relative particle populations were obtained using optical microscopy, giving the results summarised in Table 12. It is important to note that the concentration was not conserved between fractions, and hence direct comparisons between the concentrations cannot be made. However, the ratio of $2 \mu \mathrm{m}$ beads to $5 \mu \mathrm{m}$ beads can be compared, as this should be independent of the dilution.

\begin{tabular}{|c|c|c|c|}
\hline Fraction & $2 \mu \mathrm{m}$ beads & $5 \mu \mathrm{m}$ beads & Ratio $2 \mu \mathrm{m} / 5 \mu \mathrm{m}$ \\
\hline Original & 1069 & 176 & 6.1 \\
\hline$S 1$ & 70 & 14 & 5 \\
\hline$S 2$ & 57 & 20 & 2.9 \\
\hline$U 1$ & 670 & 60 & 11.2 \\
\hline$U 2$ & 285 & 43 & 6.6 \\
\hline
\end{tabular}

Table 12: Comparison of bead populations in fractions collected after magnetophoresis performed. Particle counting was performed using optical microscopy.

The original solution contained around 6 times as many $2 \mu \mathrm{m}$ beads as $5 \mu \mathrm{m}$ beads. This is because equal volumes were used, despite the expectation that the smaller beads would be supplied from the manufacturer at a higher number concentration. The lack of information regarding the concentrations of the beads in each fraction prevents us quoting an overall reduction in the ratio due to separation, as an average must take into account the size of the contribution from each fraction. We can however see that particles from $S 2$ possess half the ratio of the original solution, indicating that 
preferential selection of the larger particles has occurred. The ratio for $S 1$ is only slightly lower than the original solution, suggesting that heavier particles are preferentially settling in the fluid connectors. This was also observed in Section 4.4.3, where lighter nonmagnetic particles escaped the connectors more easily than the magnetic beads. Finally, we see that the ratio is increased for both unseparated fractions, with similar inflation of the ratio for sedimented particles again observed.

In designing the size selection experiment, it was assumed that the particles do not interact. In practice there are multiple forms of interaction, all of which may reduce the selectivity of the method. Firstly, because the bead concentration is fairly high, we would expect collisions to frequently occur. As the magnet pulls the $5 \mu \mathrm{m}$ beads across the channel, $2 \mu \mathrm{m}$ beads may be pushed in the same direction through these collisions. Secondly, the $2 \mu \mathrm{m}$ beads may form clusters in the magnetic field, which would be more mobile than individual beads. Finally, the smaller beads may bind to the larger ones through attractive magnetic forces, allowing them to 'hitchhike' into the collection stream.

\subsubsection{Separation in viscous buffer solutions}

It was observed in most of the separation experiments performed that a substantial number of particles could become pinned to the channel wall in the vicinity of the magnet. This is undesirable for the following reasons:

- Pinned particles will not be recovered at the device outlets, and will therefore be counted as lost.

- The presence of magnetic particles on the channel wall may alter the effective field gradient in the channel, causing the magnetic separation efficiency to become time-dependent.

- In extreme cases particles could build up and block the channel, preventing fluid flow altogether.

Particle pinning may occur if the field gradient is not constant over the width of the channel. Particles closer to the magnet will then experience a greater force than those which are further away. However, in most experiments the magnetic field essentially 
decayed linearly over the length scale of the channel, so this effect should be minimal. Particle agglomeration may also lead to pinning, as clusters will move across the channel more rapidly than single particles. Finally, the use of pressure-driven flow leads to a parabolic flow profile in the channel, as discussed in Section 2.3.5. This will reduce the ability of the fluid to free trapped particles, as the velocity is close to zero at the channel walls.

One potential method to suppress the pinning of particles to the channel walls is to increase the viscosity of the buffer solution, as discussed by Xia et al. [113]. As shown in Equation (31), the speed at which particles can be drawn across the channel by the magnetic field is inversely proportional to the fluid viscosity. If the buffer is more viscous than the original solution, particles which are drawn towards the magnet will be slowed down once they enter the buffer stream. The greater the difference in viscosity between the original and buffer streams, the greater the difference in the velocities of the particles. This allows particles which take longer to enter the buffer stream to 'catch up' with those that enter first, thereby reducing the spread in particle position in the buffer.

According to Equation (14), the pressure gradient in a microfluidic channel is balanced by the product of the viscosity and the laplacian of the velocity of the fluid. The pressure gradient is conserved at a given flow rate, meaning that if the viscosity is increased the laplacian term must be reduced to compensate. The laplacian must be reduced at all points in the channel, while still maintaining a parabolic flow profile. This requirement is satisfied by a reduction in the velocity of the fluid.

If two fluids of different viscosities are caused to perform laminar flow through the same channel, they will flow side by side at different linear velocities. If a syringe pump is programmed to input both fluids at the same volumetric flow rate, volume is conserved by increasing the width of the more viscous stream. This effect is seen in Figure 90, in which an aqueous solution of polyethylene glycol (PEG 200) flowed in parallel with a solution of food dye. The viscosity of the PEG increases with concentration, meaning the stream will occupy a greater proportion of the channel. Once the outlet junction is reached the two fluid streams returned to their original widths. This occurred due to the use of withdrawal syringes at the outlets, which forced the flow rates at each outlet to be 
equal. If no pressure was applied at the outlets, much of the PEG would exit at the outlet reserved for the dye.

5 vol\% PEG
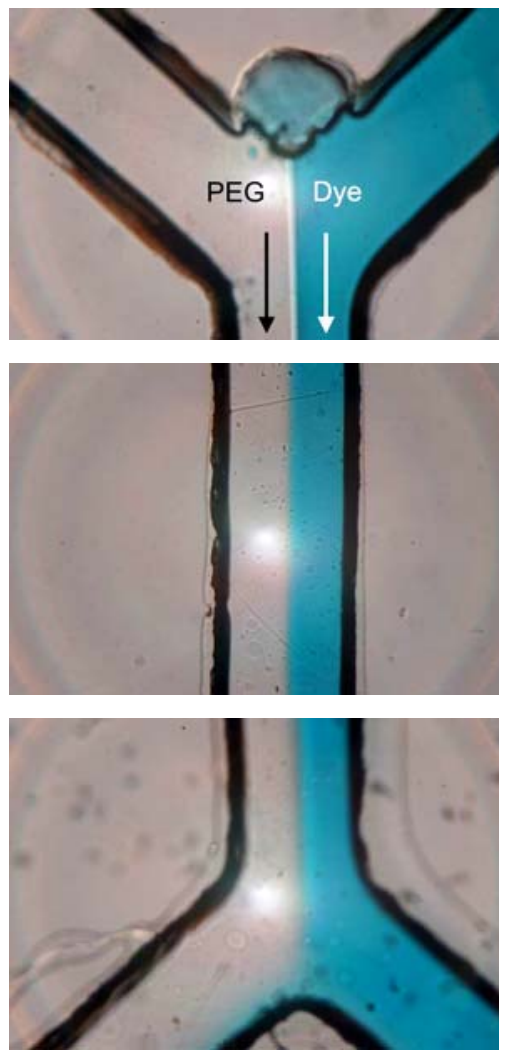

30vol\% PEG
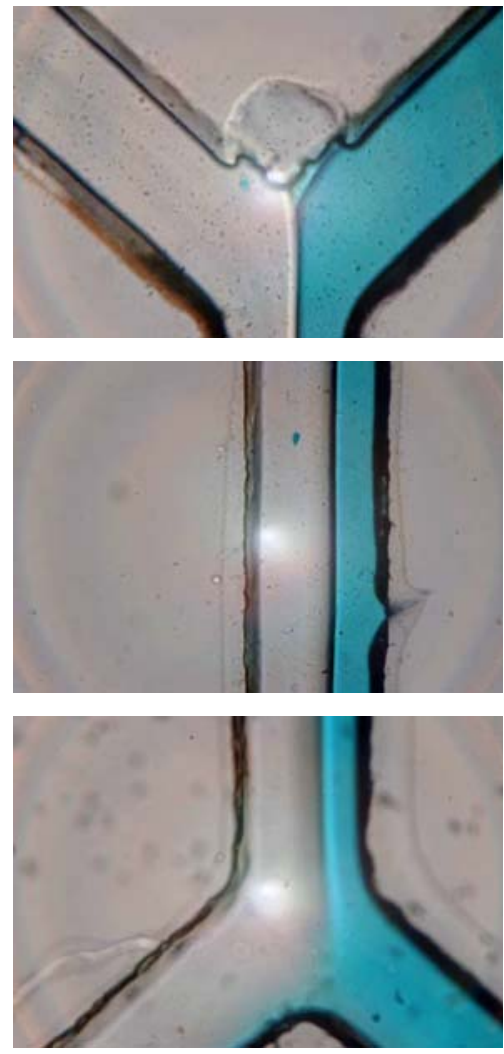

$50 \mathrm{vol} \%$ PEG
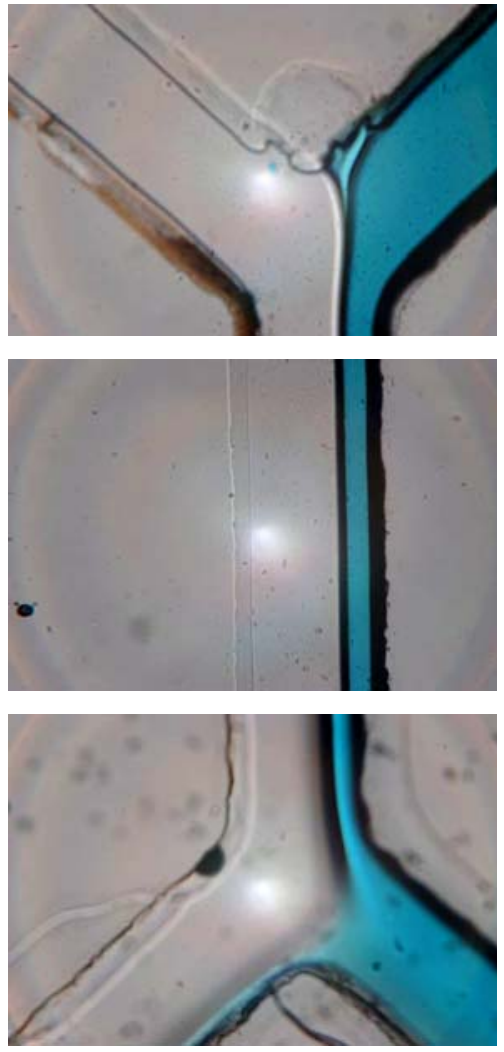

Figure 90: Laminar flow of food dye against PEG 200 at 5vol\% (left), 30vol\% (centre) and 50vol\% (right). The flow rate was $110 \mu \mathrm{L} / \mathrm{min}$, with negative pressure applied at the outlets. Images were taken at the input junction (top), main channel (centre) and outlet junction (bottom), using a $4 \mathrm{x}$ microscope objective.

The effect of a viscous buffer medium on the behaviour of magnetic particles in the channel was next investigated. A Dynabeads dispersion containing approximately $7 \times 10^{9}$ beads/mL was passed through the channel in a field gradient of $60 \mathrm{~T} / \mathrm{m}$. PEG 200 was used as the sample buffer instead of water. The PEG had a viscosity of $50 \mathrm{mPa} \cdot \mathrm{s}, 50$ times greater than that of water. The movement of magnetic beads was observed directly using a microscope, and the flow rate adjusted to optimise the separation efficiency. 
$15 \mu \mathrm{L} / \mathrm{min}$
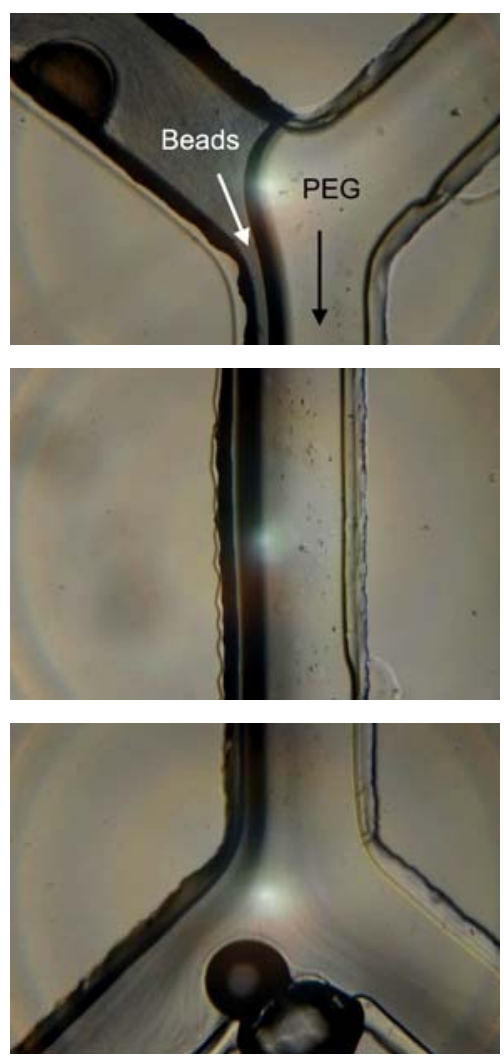

$5 \mu \mathrm{L} / \mathrm{min}$
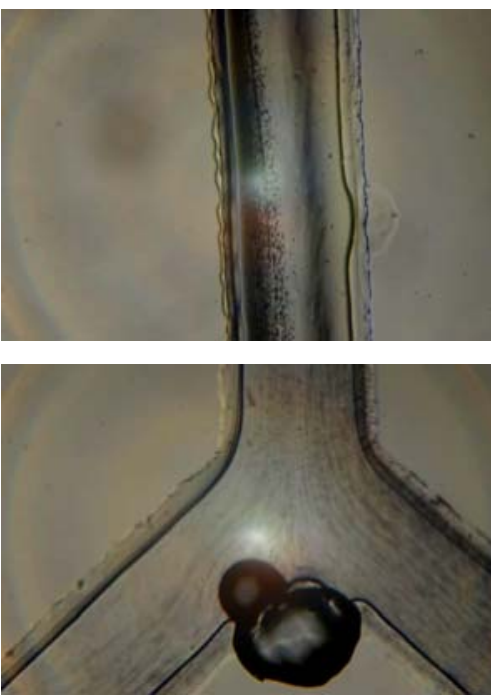

$3 \mu \mathrm{L} / \mathrm{min}$
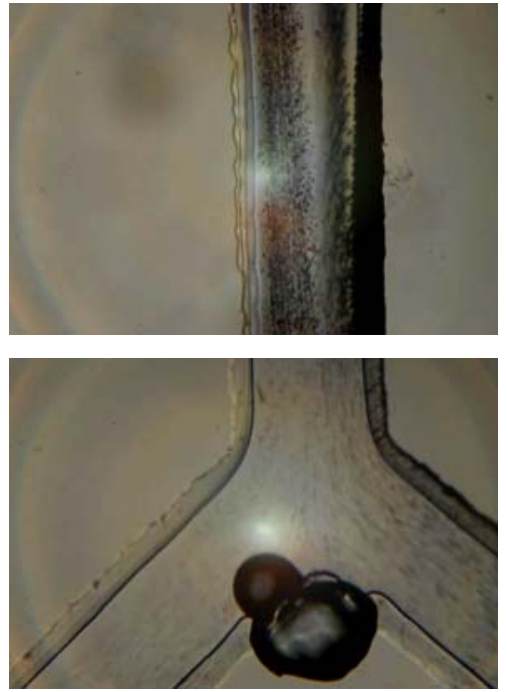

Figure 91: Magnetic separation of an aqueous dispersion of Dynabeads in a field gradient of $60 \mathrm{~T} / \mathrm{m}$. The buffer solution was undiluted PEG 200. The process was observed at flow rates of $15 \mu \mathrm{L} / \mathrm{min}$ (left), 5 $\mu \mathrm{L} / \mathrm{min}$ (centre) and $3 \mu \mathrm{L} / \mathrm{min}$ (right). Images were taken at the input junction (top), main channel (centre) and outlet junction (bottom), using a $4 \mathrm{x}$ microscope objective.

As seen in Figure 91, the beads were tightly constrained against the sidewall as they entered the channel. This was due to the extra space taken up by the viscous PEG solution. At a flow rate of $15 \mu \mathrm{L} / \mathrm{min}$, only a faint cloud of particles was seen moving into the buffer stream, the rest remaining in the original stream. Reducing the flow rate to $5 \mu \mathrm{L} /$ min allowed a larger proportion of particles to migrate into the buffer stream. Particles which had been drawn to the far side of the channel were highly localised, and moved down the channel in a thin plume. This is in contrast to separation using a water buffer, where the captured particles tended to be spread across the full width of the buffer stream. This suggests that the use of a viscous buffer medium successfully reduced variation in the positions of particles after passing the magnet. Further reducing the flow rate to $3 \mu \mathrm{L} /$ min caused many of the particles to begin accumulating on the sidewall. 
A possible drawback of the method used is that particles may be brought into close contact at the interface between the fluid streams, where particles moving rapidly across the water stream may 'overtake' those that have just entered the more viscous stream. This contact may promote agglomeration of some particles. Material can be seen peeling away from the original solution stream in large clusters in Figure 91. Agglomerates of this size were not seen when a water buffer was used, perhaps because particles are not slowed down at the fluid interface.

Quantitative measurements of the magnetic separation efficiency were carried out at a flow rate of $5 \mu \mathrm{L} / \mathrm{min}$, using a reduced bead concentration of $5 \times 10^{8}$ beads $/ \mathrm{mL}$. Only solution that exited the device was collected for testing, meaning any sedimented particles were lost. The results in Table 13 show that about $27 \%$ of the original particles exited at each outlet. This corresponds to a magnetic separation efficiency of $50 \%$, lower than that demonstrated using a water buffer in Section 4.4.2. This is also lower than we may have expected based on the results in Figure 91. The images appear to show more than half of the particles exiting the device at the buffer outlet. The reduced efficiency in quantitative measurements is likely to be due to the lower concentration of particles, which would slow the rate of particle agglomeration. Clusters of particles are drawn across the channel more rapidly, which can inflate the separation efficiency at high concentrations.

\begin{tabular}{|c|c|c|}
\hline Fraction & Concentration (beads $/ \mathrm{mL})$ & \% of original solution \\
\hline$S 1$ & $1.40 \times 10^{8}$ & 27.2 \\
\hline$U 1$ & $1.41 \times 10^{8}$ & 27.2 \\
\hline
\end{tabular}

Table 13: Concentrations of fractions collected after magnetic separation of Dynabeads at $5 \mu \mathrm{L} / \mathrm{min}$ in a field gradient of $60 \mathrm{~T} / \mathrm{m}$. PEG 200 was used as the buffer instead of water.

The results collected suggest that the use of a viscous buffer medium necessitates a reduction in flow rate, or an increase in the magnetic field gradient, in order to maintain the separation efficiency. Nonetheless, unwanted trapping of the particles does appear to be reduced. This method would be of particular use in magnetic bioseparation, in which magnetic beads bind to varying numbers of cells before being collected. Beads bound to 
multiple cells will move more slowly than those bound to a single cell, due to their increased fluid drag. Using a viscous buffer should allow all the beads to be collected, without the faster-moving ones becoming pinned.

\subsubsection{Separation of synthesised magnetic nanoparticles}

The original goal of this project was to extract iron carbide and iron oxide nanoparticles from a reaction mixture. The reaction mixture contained organic solvents, which were found to cause the separation device to fail due to swelling of the PDMS. Therefore the device could not be used for this purpose without modification of the channel surfaces. In place of this, preliminary experiments were performed using a mixture of iron and iron carbide nanoparticles, which had been recapped to allow dispersion in polar solvents.

The $20 \mathrm{~nm}$ iron and iron carbide nanoparticles were expected to be more difficult to separate than the micron-sized beads, due to their much smaller size. The nanoparticles feel a greater magnetic force per unit volume than the microbeads, due to their greater magnetic content. However, this only partly compensates for the much greater drag force per unit volume that they experience. We may estimate the velocity of a single nanoparticle in the field gradient using Equation (31)

$$
\begin{gathered}
u_{\text {mag }}=\frac{\left(4.19 \times 10^{-24} \mathrm{~m}^{3}\right)\left(3.5 \times 10^{5} \mathrm{Am}^{-1}\right)\left(60 \mathrm{Tm}^{-1}\right)}{6 \pi\left(1 \times 10^{3} \mathrm{~Pa} \cdot \mathrm{s}\right)\left(1 \times 10^{-8} \mathrm{~m}\right)} \\
=4.67 \times 10^{-7} \mathrm{~ms}^{-1} .
\end{gathered}
$$

Individual nanoparticles will move at a speed of less than $0.5 \mu \mathrm{m} / \mathrm{s}$ in the field gradient, meaning on average it would take 330 seconds to cross into the buffer stream. Complete separation would then require an extremely slow flow rate of $1 \mu \mathrm{L} /$ hour. In reality the particles would be expected to form larger agglomerates when exposed to the field, enabling them to be captured much more quickly. Additionally, the replacement of the surfactant molecules to allow dispersion in $\mathrm{EtOH}$ is likely to have formed permanent aggregates, rather than keeping the nanoparticles separate. Unfortunately it is not trivial to predict the size of these agglomerates, making the use of Equation (31) unsuitable in this case. It was instead preferred to use a slow but practical flow rate and investigate the separation efficiency visually, by using a high concentration solution. 
A solution containing approximately $6 \times 10^{13}$ particles $/ \mathrm{mL}$ in ethanol was exposed to a 60 $\mathrm{T} / \mathrm{m}$ field gradient. A constant flow rate of $5 \mu \mathrm{L} / \mathrm{min}$ was used. Using a much slower flow rate carried the risk of particles beginning to settle during the run, depending on the stability of the dispersion.

5 mins
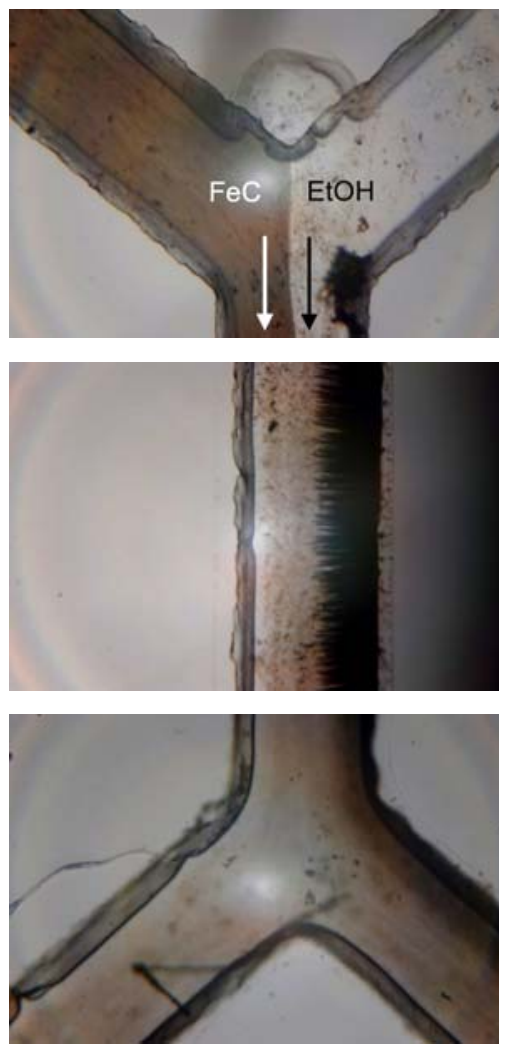

$10 \operatorname{mins}$
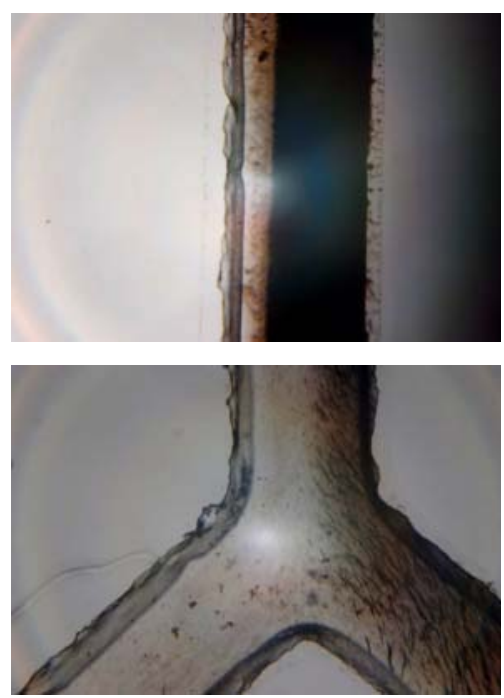

$70 \mathrm{mins}$
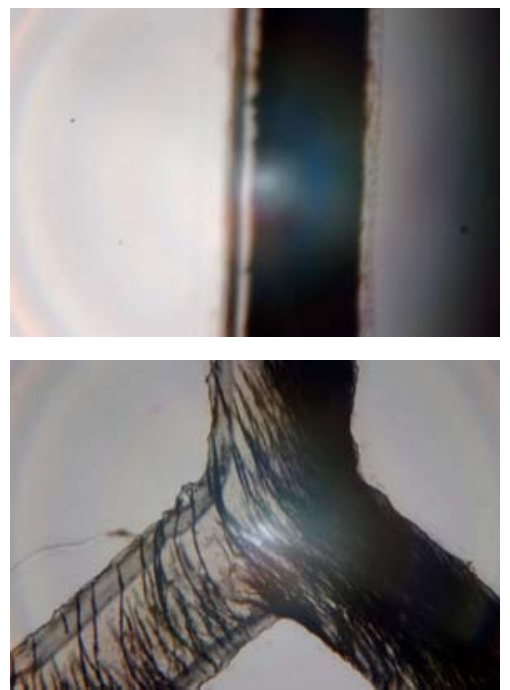

Figure 92: Magnetic separation of iron/iron carbide nanoparticles dispersed in EtOH. A flow rate of 5 $\mu \mathrm{L} / \mathrm{min}$ and field gradient of $60 \mathrm{~T} / \mathrm{m}$ were used. The process was observed 5 minutes (left), 10 minutes (centre) and 70 minutes (right) after the flow was started. Images were taken at the input junction (top), main channel (centre) and outlet junction (bottom), using a $4 \mathrm{x}$ microscope objective.

Figure 92 shows the behaviour of nanoparticles in the channel at different stages of the experiment. Even though the flow rate was substantially higher than that needed to separate individual nanoparticles, the majority of the particles appeared to be drawn into the buffer stream. This implies that substantial particle aggregation does occur. A larger number of particles accumulated at the sidewall next to the magnet, and formed into chains resembling iron filings. These chains were orientated in the direction of the magnetic field lines. Several minutes later the accumulation of the particles had 
increased, and some of the chains began to break away from the channel wall. These chains remained intact as they exit the channel, despite having exited the magnetic field. Because the particles are ferromagnetic, they have a finite remanence field. This field maintained the attraction between the particles in the chain. By the time the experiment had nearly completed, larger number of the chains were seen moving downstream of the magnet. Many of the chains exit at the side opposite to the magnet, possibly because they are crowded out by the particles built up in the other outlet.

Fractions were collected following the completion of the experiment, and attempts were made to measure the amount of magnetic material present by mass measurement. Unfortunately, only the fraction $S 2$ contained a measurable amount of material. This fraction accounted for just $20 \%$ of the material in the original solution. All remaining fractions contained too little material to measure, suggesting that an unusually large number of particles became trapped in the channel, from which a sample was not taken. This trapping may occur due to the formation of particle chains, which were not observed in experiments with other particles.

\subsubsection{Separation using Metglas system}

A small number of proof-of-concept experiments were performed using the Metglas system. As discussed in Section 3.1.6, this device was designed to allow the field gradient in the channel to be switched on and off using an external magnetic field. The soft magnetic material embedded in the device did not need to be removed in order to release the captured magnetic particles.

Quantitative measurements of the magnetic separation efficiency were carried out at a flow rate of $5 \mu \mathrm{L} / \mathrm{min}$, using a bead concentration of $7 \times 10^{8}$ beads $/ \mathrm{mL}$. Particles accumulated at the tips of the Metglas strips, but not in between them. This suggests that the strips concentrated magnetic flux from the magnet as intended. Particles had also accumulated against the channel wall downstream, and also against the wall of the luer connector. This provided evidence that the magnet itself is attracting the particles. The magnet alone provides a relatively small field gradient of $5 \mathrm{~T} / \mathrm{m}$, but this field would be felt along the full length of the channel. The field gradient from the Metglas, by comparison, was highly localised. 

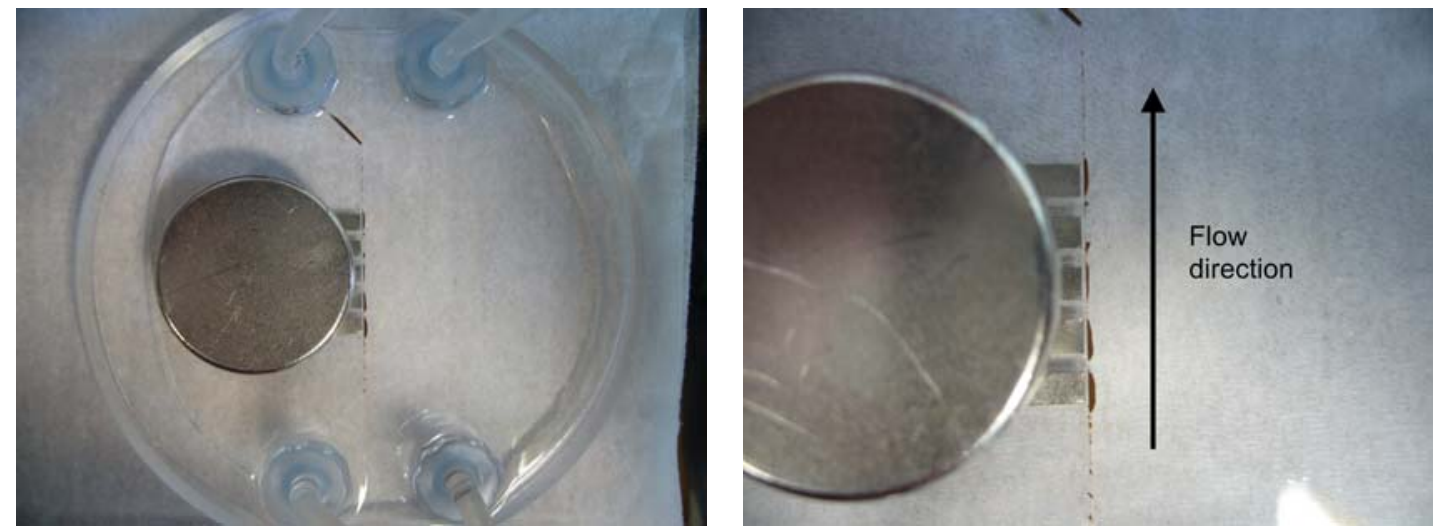

Figure 93: Metglas device immediately following magnetic separation of Dynabeads at $5 \mu \mathrm{L} / \mathrm{min}$. The magnet was present to magnetise the Metglas strips.

Measurements of the bead concentration are summarised in Table 14. Around $38 \%$ of the particles were collected in the buffer, compared to about $27 \%$ which remained in the original solution stream. The remainder was lost in the inlet tubing or within the channel. These results are inferior to the best results obtained using the magnetmicrometer system, probably due to the smaller field gradient that the Metglas system provides. The ratio of $S 1$ to $S 2$ is especially low, most likely due to the attraction between particles in the luer adaptors and the magnet.

\begin{tabular}{|c|c|c|}
\hline Fraction & Concentration (beads $/ \mathrm{mL})$ & $\%$ of original solution \\
\hline$I$ & $4.41 \times 10^{7}$ & 6.4 \\
\hline$S 1$ & $6.66 \times 10^{6}$ & 1.0 \\
\hline$S 2$ & $2.56 \times 10^{8}$ & 37.0 \\
\hline$U 1$ & $1.15 \times 10^{8}$ & 16.6 \\
\hline$U 2$ & $6.98 \times 10^{7}$ & 10.1 \\
\hline
\end{tabular}

Table 14: Concentrations of fractions collected from the Metglas device after separation of Dynabeads at $5 \mu \mathrm{L} / \mathrm{min}$.

In order to determine how much of the separation was due to the magnet rather than the Metglas, the experiment was repeated with only the magnet present. The results displayed in Table 15 show that only around $14 \%$ of the particles are collected, while a further $21 \%$ remain in the original stream. The amount of particles settling in the inlet tubing has tripled, although the cause of this is not known. One possibility is that the 
tubing was in closer proximity to the magnet, which could cause the particles to be attracted towards the walls of the tubing. If we ignore the lost particles, the magnetic separation efficiency is decreased from $58 \%$ to $40 \%$ when the Metglas elements are removed, confirming that the elements do create stronger field gradients than the magnet alone.

\begin{tabular}{|c|c|c|}
\hline Fraction & Concentration $($ beads $/ \mathrm{mL})$ & \% of original solution \\
\hline$I$ & $2.46 \times 10^{8}$ & 37.6 \\
\hline$S 1$ & $2.05 \times 10^{7}$ & 3.1 \\
\hline$S 2$ & $6.92 \times 10^{7}$ & 10.6 \\
\hline$U 1$ & $7.07 \times 10^{7}$ & 10.8 \\
\hline$U 2$ & $6.35 \times 10^{7}$ & 9.7 \\
\hline
\end{tabular}

Table 15: Concentrations of fractions collected from control device after separation of Dynabeads at 5 $\mu \mathrm{L} / \mathrm{min}$. 


\section{Discussion and Conclusions}

This work describes the design, development and evaluation of microfluidic devices for the separation of magnetic particles. Although the main results relate to the use of a permanent magnet system for the separation of magnetic microbeads, multiple device designs were developed and tested with a range of particle types. In this section the most important achievements and results are summarised and discussed, and possibilities for future work are suggested.

\section{Characterisation of the magnetic properties of magnetic microbeads and nanoparticles}

Magnetic nanoparticles used in this project were synthesised using a novel method developed by Cheong [39], and their magnetic properties were not understood in much detail. It was therefore important to measure these properties directly, so that appropriate conditions could be set for effective magnetic separation. Magnetic nanoparticles produced by solution-phase synthesis were composed of a large number of $5 \mathrm{~nm}$ iron oxide particles, and a smaller number of $20 \mathrm{~nm}$ iron and iron carbide particles. VSM and SQUID measurements lead to the conclusion that the iron oxide particles were superparamagnetic. This is consistent with the prediction made in Section 2.1.4, using Equation (8), that magnetite particles become superparamagnetic at diameters below 14 nm. By contrast, VSM measurements showed that the iron and iron carbide particles were ferromagnetic. Although superparamagnetic particles are typically favoured for biomedical applications, the large saturation magnetisation of the iron and iron carbide nanoparticles may make them more suitable for some uses.

Manufacturers of magnetic microbeads also provide minimal information about the magnetic properties of their products, perhaps because they are usually used in test tubebased experiments, for which knowledge of the exact magnitude of the magnetisation is not required. Magnetic microbeads are designed to be superparamagnetic, so that captured beads may be easily redispersed after capture. VSM measurements supported

the manufacturers' claims that both Dynabead and Spherotech particles are superparamagnetic, as the measured coercivity for each type was less than that of the 
iron oxide nanoparticles. The saturation magnetisation of all bead types was substantially less than that of the synthesised nanoparticles, which is expected because the beads are composed mainly of nonmagnetic polystyrene, with only a small amount of magnetic content. It was also observed that the magnetisation increases with decreasing bead size. It is likely that the manufacturers increase the magnetic content of smaller beads, to compensate for the increasing significance of fluid drag.

\section{Development of a process for producing microfluidic devices in PDMS}

Although the essential techniques for rapid prototyping of PDMS microfluidic devices had been previously established [152], further development and refinement of these methods was an important aspect of this project. In particular, improving the durability of the devices was crucial to allowing in-depth characterisation of their use in magnetic separation. A number of alterations were made to the standard fabrication procedures, which helped to improve the fidelity and robustness of the devices produced.

Laser cutting of the PMMA into wafers for SU-8 lithography greatly increased the quality and durability of the resulting masters, as the cleaner surface improved the strength of the bond between the SU-8 and PMMA. Fluid connectors were integrated directly into the sealing PDMS layer of the devices, thus avoiding the need to cast the connectors into an additional PDMS layer and bond it to the device. This improved fabrication time, prevented leaks forming, and reduced the dead volume of each device. Increasing the thickness of the PDMS to accommodate the connectors also had the benefit of improving the mechanical strength of the devices.

While the dead volume of the devices has been reduced, the use of luer adaptors as fluid connectors meant the volume was still fairly large. Each connector has an internal volume of approximately $100 \mu \mathrm{L}$, resulting in a total dead volume of $200 \mu \mathrm{L}$ for each fluid stream. The fluid channel itself has a total volume of just $2 \mu \mathrm{L}$, meaning further reductions in dead volume should be pursued. This could most simply be achieved by defining holes in the top PDMS layer in place of the luers. Tubing could then be inserted directly into these holes, relying on the elasticity of the PDMS to create a seal. However, this connection would be likely to fail at high pressures. A more reliable connection could be formed by replacing the luer connectors with Nanoports (Upchurch [204]), 
which have zero dead volume. While Nanoports are designed to be bonded to glass, techniques to embed the connectors into PDMS are already present in the literature [205].

The channels of the microfluidic devices made for this project were no smaller than 300 $\mu \mathrm{m}$, meaning limitations imposed on channel resolution by the photolithography process were of little importance. In applications requiring narrower channels however, it may be necessary to improve the quality of the photomasks, as well as the UV-exposure process. The resolution of the photomasks could be easily improved by using a higher resolution printer. Alternatively glass or chrome photomasks could be used, although this would greatly increase cost. Replacing the UV-source used with a collimated source would also be expected to improve the verticality of the sidewalls.

It was found that the swelling of PDMS in organic solvents prevented devices from being used with particles dispersed in toluene. Although not attempted in the course of this project, modifications can be made to PDMS to improve its solvent resistance. Abate et al. [202] coated the interior walls of PDMS microchannels with a layer of glass, by flowing a sol-gel solution through the channel. This produced channels with the excellent chemical resistance of glass, without requiring the complicated machining techniques used to produce all-glass systems. Other possibilities include polymer coatings [206], or doping the PDMS with zeolites to increase cross-linking [207].

\section{Observation of the flow properties of PDMS microfluidic devices}

It was visually confirmed in Figure 73 that the flow of liquid inside the channels was laminar. This was a crucial requirement for magnetic separation, as the solution containing the particles needed to be brought into contact with a buffer solution without mixing. Spectroscopic measurements showed that a dye solution mixed with the buffer by diffusion alone, but mixing was observed for a solution containing particles. This implies that there is a secondary mechanism by which particles are transferred into the buffer stream. Although this mechanism is not known, it has been suggested [201] that particles may induce localised turbulence in fluids, which could promote mixing. 


\section{Development of magnetic separation systems using permanent magnets}

Two magnetic separation systems based on permanent magnets were designed during this project. In the first of these two designs, small magnets were embedded in the PDMS. The magnets could be brought within $335 \mu \mathrm{m}$ of the channel without leaks occurring, meaning magnetic field gradients of up to $500 \mathrm{~T} / \mathrm{m}$ could be produced in the channel. However, because the magnets were permanently embedded inside the device, it was impossible to adjust this field gradient. This meant that if particles became trapped inside the channel, the only method for recovery was to flush with a buffer at very high flow rates, which could damage the device. The separation efficiency of this device was not extensively characterised, mainly due to this limitation. Because the embedded magnet devices were inexpensive to produce, they may be well suited for single-use applications, for which there is usually no need to clean the channels after use.

The second device design was developed to allow the magnetic field gradient to be removed or adjusted. The design consisted of a single small magnet mounted on a wire arm. This arm could be precisely moved using a micrometer. A notch was cut into the PDMS microfluidic device, allowing the magnet to be brought closer to the channel. However, the magnet could only be brought within $2.3 \mathrm{~mm}$ of the channel wall, which reduced the maximum field gradient to $60 \mathrm{~T} / \mathrm{m}$. It should be possible in future to extend the notch closer to the channel, which would substantially improve the strength of the field gradient. It was difficult to cut close to the channel without pulling the two PDMS layers apart, so it would be preferable to cut the two layers before sealing them together. This will require a procedure to ensure that the notches cut into each piece are properly aligned.

\section{Evaluation of the ability to separate magnetic microbeads and nanoparticles}

\section{using a permanent magnet system}

A number of experiments to evaluate the ability of the magnet-micrometer system to separate magnetic microbeads were performed. It was shown that in zero field most of the beads remained in the original fluid stream, but as the field gradient was increased more of the beads would migrate into the buffer stream. Reducing the flow rate also increased the number of particles captured, by exposing them to the magnetic field for a 
longer period. Larger concentrations of particles were also captured more easily, because the particles can form into clusters. Spectroscopic measurements showed that Dynabead MyOne magnetic beads could be separated with an efficiency of $75 \%$, provided particles lost to sedimentation were ignored. Interestingly, around $13 \%$ of the particles were still captured without a magnetic field present. This non-selective separation may occur due to localised turbulence at the boundary between the two fluid streams, although this has not been confirmed.

Preliminary experiments to separate water-dispersible iron and iron carbide nanoparticles using the same system resulted in limited success. As shown in Figure 92, a large proportion of the particles were drawn into the buffer stream by the magnet, despite the experimental flow rate being around 300 times larger than the theoretical rate required. It is thought that the particles agglomerated rapidly in the magnetic field, allowing them to be drawn across the channel much faster than if the nanoparticles moved independently. It was observed that over time the nanoparticles began to form long chains, which were aligned with the field lines of the magnet. Because the particles were not superparamagnetic, these chains did not break apart once they had formed. Most of the particles therefore became trapped inside the channels, preventing the separation efficiency from being measured.

A major drawback of the current system is that a large proportion of the particles are lost through sedimentation in the device. This is mainly due to the large dead volumes of the tubing and luer connectors. The flow velocity in these volumes is very slow, making sedimentation due to gravity more likely. Replacing the luer connectors with zero-deadvolume connectors such as Nanoports would help to counteract this problem, as would reducing the diameter of the solution tubing.

\section{Evaluation of the ability to select microbeads on the basis of magnetophoretic mobility using a permanent magnet based system}

A limited ability to separate microbeads according to their magnetisation has been demonstrated. A solution containing nonmagnetic and magnetic beads, both $1 \mu \mathrm{m}$ in diameter, was purified using the magnet-micrometer system. The ratio of nonmagnetic beads to magnetic beads was reduced from 5.3 to 2.7 in a single separation step. Further 
purification should be achievable through repeat separation steps. It was found that the magnetic beads settled in the luer adaptors more frequently than the much lighter nonmagnetic beads, and this effect tended to skew the results. Eliminating the dead volume of the fluid connections, as discussed previously, should help to reduce sedimentation.

Microbeads were also separated according to their size. A solution containing magnetic beads $2 \mu \mathrm{m}$ and $5 \mu \mathrm{m}$ in diameter was purified using the magnet-micrometer system. The ratio of $2 \mu \mathrm{m}$ beads to $5 \mu \mathrm{m}$ beads in the solution was decreased from 6.1 to as low as 2.9. Unfortunately, using microscopy to determine the ratio in collected fractions prevented an overall ratio being found, as the absolute concentration of each fraction is unknown. Improved results could be achieved if an alternative measurement system, sensitive to both the concentration and size of particles, could be used.

\section{Use of viscous solutions to improve separation uniformity}

Experiments were performed using PEG-200 as a buffer for the separation of magnetic

microbeads. It was hoped that the increased fluid drag associated with the viscosity of the PEG would prevent beads becoming pinned against the sidewall of the microfluidic channel. Visual observations confirmed that the beads moved into the buffer stream in a much tighter group than when a water buffer was used, making it easier to prevent particles becoming trapped in the channel.

A drawback of this method was that the sudden change in fluid viscosity at the boundary appears to promote the formation of large clusters of particles, which travel much more slowly across the channel than individual particles. Reducing the viscosity of the buffer stream would be expected to reduce the severity of this effect. However, further work is needed to determine the minimum reduction in viscosity needed to reduce agglomeration to acceptable levels.

\section{Development and evaluation of a magnetic separation system using soft magnetic materials}

A magnetic separation system using strips of the amorphous metal Metglas was developed. It was hoped that a uniform magnetic field could be used to magnetise the 
strips, which would then create a strong magnetic field gradient in the channel. Unfortunately a uniform field of sufficient strength was not achieved, making it necessary to instead use a permanent magnet to saturate the strips. Although the expected field gradient from the Metglas of 25-60 T/m was much larger than the $5 \mathrm{~T} / \mathrm{m}$ from the magnet, the field gradient from the magnet extended over a much greater distance, allowing it to attract particles along the full length of the channel. The Metglas therefore only slightly improved the separation process compared to the magnet alone, with the efficiency increasing from $40 \%$ to $58 \%$ when the strips were present.

One of the key limitations of the device was the size of the gap between the Metglas strips and the fluid channel. The field gradient from the strips is strong but highly localised, making it important to make this gap as small as possible. Defining recesses in the PDMS for the Metglas helped to reduce this distance to $200 \mu \mathrm{m}$. However, once the width of the channel itself is taken into account, magnetic particles will be as far as 500 $\mu \mathrm{m}$ away from the Metglas and will experience only a weak field gradient.

It may be of interest in future work to develop a Metglas-based device for particle capture rather than continuous flow separation. A potential design for such a device, based on a bead capture system produced by Bu et al. [129], is presented in Figure 94. In this system the Metglas strips are placed underneath the channel rather than beside it. Making the layer of PDMS containing the channel as thin as possible should allow the Metglas to be brought extremely close to the bottom of the fluid channel. Permanent magnets placed under the device provide a long-range magnetic field gradient, which will attract magnetic particles towards the floor of the channel. The Metglas strips, activated by the field from the magnets, provide a strong short-range field gradient to capture the particles. Removing the magnets deactivates the Metglas and releases the particles, allowing them to be flushed out of the device. Because the field gradient is applied vertically, the channel can be made several centimetres wide, allowing separation to occur at much higher flow rates. As discussed in Section 2.5.2, the main drawback of such a device is that the amount of material that may be captured in a single step is limited by the capacity of the channel. 

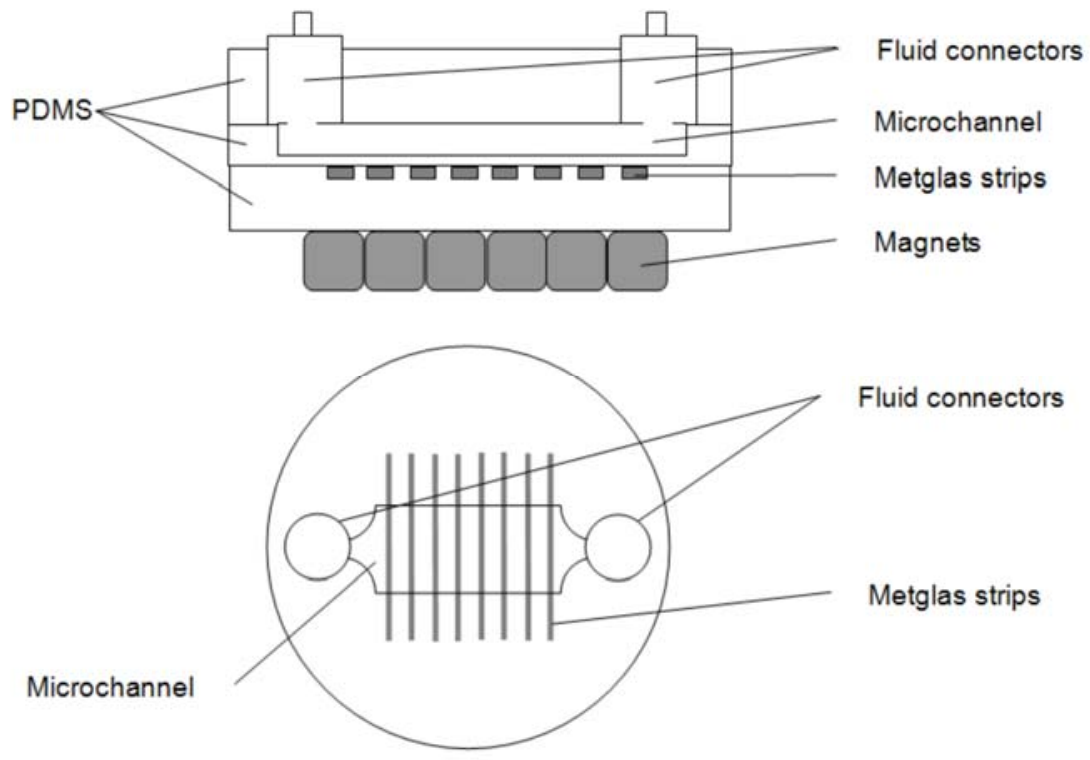

Figure 94: Proposed design for a particle capture magnetic separation system using Metglas and permanent magnets.

At the end of this study the following key conclusions may be drawn:

- Microfluidic devices have been produced using a low-cost rapid prototyping process in PDMS. These devices are robust and durable, making them suitable for long-term use. At the same time the devices are inexpensive, meaning they are also suitable for use on a disposable basis.

- Three methods for producing a magnetic field gradient in a microfluidic channel have been developed, using either permanent magnets or soft magnetic materials. Magnetic field gradients of 25-500 T/m were demonstrated, although the largest adjustable field gradient was $60 \mathrm{~T} / \mathrm{m}$.

- Microfluidic devices were applied to the continuous flow separation of magnetic particles, particularly magnetic microbeads. The separation efficiency was proportional to the strength of the magnetic field gradient and particle concentration, and inversely proportional to the flow rate. The devices were capable of separating Dynabeads MyOne magnetic beads with an efficiency of up to $75 \%$, at a flow rate of $5 \mu \mathrm{L} / \mathrm{min}$. Such a device may be suitable for biological applications such as immunoassays. 


\section{List of symbols}

In order of first appearance

\begin{tabular}{|c|c|c|}
\hline Symbol & Description & SI Unit/value \\
\hline $\mathrm{B}$ & magnetic flux density & $\mathrm{T}$ \\
\hline $\mathrm{H}$ & applied magnetic field & $\mathrm{A} \cdot \mathrm{m}^{-1}$ \\
\hline$\mu_{0}$ & magnetic permeability of free space & $4 \pi \times 10^{-7} \mathrm{~N} \cdot \mathrm{A}^{-2}$ \\
\hline $\mathrm{M}$ & magnetisation & $\mathrm{A} \cdot \mathrm{m}^{-1}$ \\
\hline$\chi$ & magnetic susceptibility & dimensionless \\
\hline $\mathrm{m}$ & magnetic moment & $\mathrm{J} \cdot \mathrm{T}^{-1}$ \\
\hline $\mathrm{k}$ & Boltzmann's constant & $1.38 \times 10^{-23} \mathrm{~J} \cdot \mathrm{K}^{-1}$ \\
\hline $\mathrm{T}$ & temperature & $\mathrm{K}$ \\
\hline $\mathrm{E}$ & energy & $\mathrm{J}$ \\
\hline \multirow{2}{*}{$\lambda$} & domain wall width & $\mathrm{m}$ \\
\hline & wavelength & $\mathrm{m}$ \\
\hline $\mathrm{K}$ & magnetocrystalline anisotropy energy density & $\mathrm{J} \cdot \mathrm{m}^{-3}$ \\
\hline $\mathrm{V}$ & volume & $\mathrm{m}^{3}$ \\
\hline$\tau$ & relaxation time & $\mathrm{s}$ \\
\hline$\alpha$ & Langevin fitting parameter & dimensionless \\
\hline $\mathrm{V}$ & velocity & $\mathrm{m} \cdot \mathrm{s}^{-1}$ \\
\hline$\rho$ & density & $\mathrm{kg} \cdot \mathrm{m}^{-3}$ \\
\hline$\eta$ & dynamic viscosity & $\mathrm{Pa} \cdot \mathrm{s}$ \\
\hline $\mathrm{p}$ & pressure & $\mathrm{N} \cdot \mathrm{m}^{-2}$ \\
\hline $\mathrm{L}$ & length & $\mathrm{m}$ \\
\hline $\mathrm{Re}$ & Reynolds number & dimensionless \\
\hline $\mathrm{D}$ & diffusion coefficient & $\mathrm{m}^{2} \cdot \mathrm{s}^{-1}$ \\
\hline $\mathrm{t}$ & time & $\mathrm{s}$ \\
\hline $\mathrm{R}$ & universal gas constant & $\mathrm{J} \cdot \mathrm{K}^{-1} \cdot \mathrm{mol}^{-1}$ \\
\hline $\mathrm{N}$ & Avagadro constant & $6.02 \times 10^{23} \mathrm{~mol}^{-1}$ \\
\hline $\mathrm{r}$ & radius & $\mathrm{m}$ \\
\hline $\mathrm{x}$ & position & $\mathrm{m}$ \\
\hline
\end{tabular}




\begin{tabular}{|l|l|l|}
\hline $\mathrm{c}$ & concentration & $\mathrm{mol} \cdot \mathrm{L}^{-1}$ \\
\hline $\mathrm{g}$ & gravitational acceleration & $9.81 \mathrm{~m} \cdot \mathrm{s}^{-2}$ \\
\hline $\mathrm{u}$ & velocity (relative) & $\mathrm{m} \cdot \mathrm{s}^{-1}$ \\
\hline $\mathrm{G}$ & magnetic field gradient & $\mathrm{T} \cdot \mathrm{m}^{-1}$ \\
\hline $\mathrm{d}$ & diameter & $\mathrm{m}$ \\
\hline Q & volumetric flow rate & $\mathrm{m}^{3} \cdot \mathrm{s}^{-1}$ \\
\hline h & height & $\mathrm{m}$ \\
\hline W & width & $\mathrm{m}$ \\
\hline I & intensity (light) & $\mathrm{W} \cdot \mathrm{m}^{-2}$ \\
\hline A & absorbance & $\mathrm{dimensionless}$ \\
\hline$\varepsilon$ & molar absorptivity & $\mathrm{m}^{2} \cdot \mathrm{mol}^{-1}$ \\
\hline
\end{tabular}




\section{References}

1. J.H.P. Watson, Magnetic filtration, Journal of Applied Physics 44, 4209-4213 (1973).

2. J. Oberteuffer, Magnetic separation: A review of principles, devices, and applications, Magnetics, IEEE Transactions on 10, 223-238 (1974).

3. G.M. Whitesides, The origins and the future of microfluidics, Nature 442, 368-373 (2006).

4. H. Bruus, Theoretical Microfluidics, (Oxford University Press, 2008).

5. D.C. Duffy, J.C. McDonald, O.J.A. Schueller \& G.M. Whitesides, Rapid prototyping of microfluidic systems in poly(dimethylsiloxane), Analytical Chemistry 70, 4974-4984 (1998).

6. D. Meeker, Finite Element Method Magnetics, Available at http://femm.foster-miller.net.

7. S. Chikazumi \& C. Graham, Physics of Magnetism, (Oxford University Press, 1997).

8. D. Halliday, R. Resnick \& J. Walker, Fundamentals of Physics, (John Wiley and Sons, 2003).

9. B.D. Cullity, Introduction to Magnetic Materials, (Addison-Wesley, 1972).

10. C. Kittel, Introduction to Solid State Physics, (Wiley, 1976).

11. C.L. Dennis et al., The defining length scales of mesomagnetism: A review, Journal of Physics: Condensed Matter 14, 1175-1262 (2002).

12. N. Spaldin, Magnetic Materials, (Cambridge University Press, 2003).

13. X. Batlle \& A. Labarta, Finite-size effects in fine particles: Magnetic and transport properties, Journal of Physics D: Applied Physics 35, 15-42 (2002).

14. S.A. Majetich \& Y. Jin, Magnetization directions of individual nanoparticles, Science 284, 470473 (1999).

15. W.F. Brown, Thermal Fluctuations of a Single-Domain Particle, Physical Review 130, 1677 (1963).

16. D.L. Leslie-Pelecky \& R.D. Rieke, Magnetic properties of nanostructured materials, Chemistry of Materials 8, 1770-1783 (1996).

17. R. Koetitz, P.C. Fannin \& L. Trahms, Time domain study of Brownian and Neel relaxation in ferrofluids, Journal of Magnetism and Magnetic Materials 149, $42-46$ (1995).

18. A.K. Arzhnikov, L.V. Dobysheva \& C. Demangeat, Structural peculiarities of cementite and their influence on magnetic characteristics, Journal of Physics Condensed Matter 19, 196214-196223 (2007).

19. A.H. Lu, E.L. Salabas \& F. Schuth, Magnetic nanoparticles: Synthesis, protection, functionalization, and application, Angewandte Chemie - International Edition 46, 1222-1244 (2007).

20. A.S. Teja \& P.Y. Koh, Synthesis, properties, and applications of magnetic iron oxide nanoparticles, Progress in Crystal Growth and Characterization of Materials 55, 22-45 (2009). 
21. P. Tartaj, M. Del Puerto Morales, S. Veintemillas-Verdaguer, T. Gonzalez-Carreno \& C.J. Serna, The preparation of magnetic nanoparticles for applications in biomedicine, Journal of Physics D: Applied Physics 36, 182-197 (2003).

22. P. Tartaj, M.P. Morales, T. Gonzalez-Carreno, S. Veintemillas-Verdaguer \& C.J. Serna, Advances in magnetic nanoparticles for biotechnology applications, Journal of Magnetism and Magnetic Materials 290-291, 28-34 (2005).

23. B.L. Cushing, V.L. Kolesnichenko \& C.J. O'Connor, Recent Advances in the Liquid-Phase Syntheses of Inorganic Nanoparticles, Chemical Reviews 104, 3893-3946 (2004).

24. Q.A. Pankhurst, J. Connolly, S.K. Jones \& J. Dobson, Applications of magnetic nanoparticles in biomedicine, Journal of Physics D: Applied Physics 36, 167-181 (2003).

25. V.K. LaMer \& R.H. Dinegar, Theory, production and mechanism of formation of monodispersed hydrosols, Journal of the American Chemical Society 72, 4847-4854 (1950).

26. C.J.J. den Ouden \& R.W. Thompson, Analysis of the formation of monodisperse populations by homogeneous nucleation, Journal of Colloid and Interface Science 143, 77-84 (1991).

27. M. Ocana, R. Rodriguez-Clemente \& C.J. Serna, Uniform colloidal particles in solution: Formation mechanisms, Advanced Materials 7, 212-216 (1995).

28. T. Sugimoto \& E. Matijevic, Formation of uniform spherical magnetite particles by crystallization from ferrous hydroxide gels, Journal of Colloid and Interface Science 74, 227-243 (1980).

29. D.K. Kim, Y. Zhang, W. Voit, K.V. Rao \& M. Muhammed, Synthesis and characterization of surfactant-coated superparamagnetic monodispersed iron oxide nanoparticles, Journal of Magnetism and Magnetic Materials 225, 30-36 (2001).

30. C.L. Lin, C.F. Lee \& W.Y. Chiu, Preparation and properties of poly(acrylic acid) oligomer stabilized superparamagnetic ferrofluid, Journal of Colloid and Interface Science 291, 411-420 (2005).

31. D.K. Kim, M. Mikhaylova, Y. Zhang \& M. Muhammed, Protective coating of superparamagnetic iron oxide nanoparticles, Chemistry of Materials 15, 1617-1627 (2003).

32. D.K. Kim et al., Starch-Coated Superparamagnetic Nanoparticles as MR Contrast Agents, Chemistry of Materials 15, 4343-4351 (2003).

33. Z.P. Xu, Q. Hua Zeng, G.Q. Lu \& A. Bing Yu, Inorganic nanoparticles as carriers for efficient cellular delivery, Chemical Engineering Science 61, 1027-1040 (2006).

34. A.L. Willis, N.J. Turro \& S. O'Brien, Spectroscopic characterization of the surface of iron oxide nanocrystals, Chemistry of Materials 17, 5970-5975 (2005).

35. A.G. Roca, M.P. Morales, K. O'Grady \& C.J. Serna, Structural and magnetic properties of uniform magnetite nanoparticles prepared by high temperature decomposition of organic precursors, Nanotechnology 17, 2783-2788 (2006).

36. K. Muraishi, T. Takano, K. Nagase \& N. Tanaka, Thermal decomposition of Fe(II) carboxylates: Comparison of decomposition processes between the formate and malonate, Journal of Inorganic and Nuclear Chemistry 43, 2293-2297 (1981).

37. C. Amiens \& B. Chaudret, Organometallic synthesis of nanoparticles, Modern Physics Letters B 21, 1133-1141 (2007). 
38. J. Sun, R. Xu, Y. Zhang, M. Ma \& N. Gu, Magnetic nanoparticles separation based on nanostructures, Journal of Magnetism and Magnetic Materials 312, 354-358 (2007).

39. S. Cheong, Personal communication, (2009).

40. E. Bourgeat-Lami \& J. Lang, Encapsulation of inorganic particles by dispersion polymerization in polar media 1. Silica nanoparticles encapsulated by polystyrene, Journal of Colloid and Interface Science 197, 293-308 (1998).

41. V.S. Zaitsev, D.S. Filimonov, I.A. Presnyakov, R.J. Gambino \& B. Chu, Physical and chemical properties of magnetite and magnetite-polymer nanoparticles and their colloidal dispersions, Journal of Colloid and Interface Science 212, 49-57 (1999).

42. E.E. Hassan, R.C. Parish \& J.M. Gallo, Optimized formulation of magnetic chitosan microspheres containing the anticancer agent, oxantrazole, Pharmaceutical Research 9, 390-397 (1992).

43. D. Muller-Schulte \& H. Brunner, Novel magnetic microspheres on the basis of poly(vinyl alcohol) as affinity medium for quantitative detection of glycated haemoglobin, Journal of Chromatography A 711, 53-60 (1995).

44. Spherotech, Sphero Magnetic Particles, Available at http://www.spherotech.com/new\%20downloadable \%20notes/Magnetic\%20Particles\%2020082009.pdf.

45. G. Fonnum, C. Johansson, A. Molteberg, S. Mørup \& E. Aksnes, Characterisation of Dynabeads ${ }^{\circledR}$ by magnetization measurements and Mössbauer spectroscopy, Journal of Magnetism and Magnetic Materials 293, 41-47 (2005).

46. M. Gijs, in Microfluidic Technologies for Miniaturized Analysis Systems (Springer, 2007), pp. 241-274.

47. M.P. Sharrock \& R.E. Bodnar, Magnetic materials for recording: An overview with special emphasis on particles (invited), Journal of Applied Physics 57, 3919-3924 (1985).

48. R.E. Camley \& R.L. Stamps, Magnetic multilayers: Spin configurations, excitations and giant magnetoresistance, Journal of Physics: Condensed Matter 5, 3727-3786 (1993).

49. R.E. Rosenweig, Ferrohydrodynamics, (Dover Publications, 1997).

50. C. Rinaldi, A. Chaves, S. Elborai, X. He \& M. Zahn, Magnetic fluid rheology and flows, Current Opinion in Colloid and Interface Science 10, 141-157 (2005).

51. J.-P. Dery, E.F. Borra \& A.M. Ritcey, Ethylene Glycol Based Ferrofluid for the Fabrication of Magnetically Deformable Liquid Mirrors, Chemistry of Materials 20, 6420-6426 (2008).

52. J.P. Dailey, J.P. Phillips, C. Li \& J.S. Riffle, Synthesis of silicone magnetic fluid for use in eye surgery, Journal of Magnetism and Magnetic Materials 194, 140-148 (1999).

53. Rare earth magnets for fun and profit, Available at http://www.dansdata.com/magnets.htm\#ff.

54. MACS Cell Separators, Available at http://www.miltenyibiotec.com.

55. Magnets for molecular and cell separation applications, Available at http://www.invitrogen.com/site/us/en/home/brands/Dynal/Magnets.html.

56. D. Tanyolac \& A.R. Zdural, BSA adsorption onto magnetic polyvinylbutyral microbeads, Journal of Applied Polymer Science 80, 707-715 (2001). 
57. L. Cumbal, J. Greenleaf, D. Leun \& A.K. SenGupta, Polymer supported inorganic nanoparticles: Characterization and environmental applications, Reactive and Functional Polymers 54, 167-180 (2003).

58. D.K. Kim et al., Characterization and MRI study of surfactant-coated superparamagnetic nanoparticles administered into the rat brain, Journal of Magnetism and Magnetic Materials 225, 256-261 (2001).

59. A. Bjørnerud \& L. Johansson, The utility of superparamagnetic contrast agents in MRI: Theoretical consideration and applications in the cardiovascular system, NMR in Biomedicine 17, 465-477 (2004).

60. S. Mornet, S. Vasseur, F. Grasset \& E. Duguet, Magnetic nanoparticle design for medical diagnosis and therapy, Journal of Materials Chemistry 14, 2161-2175 (2004).

61. C. Sun, J.S.H. Lee \& M. Zhang, Magnetic nanoparticles in MR imaging and drug delivery, Advanced Drug Delivery Reviews 60, 1252-1265 (2008).

62. S. Goodwin, C. Peterson, C. Hoh \& C. Bittner, Targeting and retention of magnetic targeted carriers (MTCs) enhancing intra-arterial chemotherapy, Journal of Magnetism and Magnetic Materials 194, 132-139 (1999).

63. R.E. Rosensweig, Heating magnetic fluid with alternating magnetic field, Journal of Magnetism and Magnetic Materials 252, 370-374 (2002).

64. A. Jordan, R. Scholz, P. Wust, H. Fahling \& R. Felix, Magnetic fluid hyperthermia (MFH): Cancer treatment with AC magnetic field induced excitation of biocompatible superparamagnetic nanoparticles, Journal of Magnetism and Magnetic Materials 201, 413-419 (1999).

65. Gad-el-Hak, The MEMS Handbook: MEMS, Design and Fabrication, (CRC Press, 2006).

66. P.A. Auroux, Y. Koc, A. DeMello, A. Manz \& P.J.R. Day, Miniaturised nucleic acid analysis, Lab on a Chip - Miniaturisation for Chemistry and Biology 4, 534-546 (2004).

67. I. Inoue, Y. Wakamoto, H. Moriguchi, K. Okano \& K. Yasuda, On-chip culture system for observation of isolated individual cells, Lab on a Chip - Miniaturisation for Chemistry and Biology 1, 50-55 (2001).

68. S.W. Rhee et al., Patterned cell culture inside microfluidic devices, Lab on a Chip Miniaturisation for Chemistry and Biology 5, 102-107 (2005).

69. N. Pamme, Magnetism and microfluidics, Lab on a Chip - Miniaturisation for Chemistry and Biology 6, 24-38 (2006).

70. P. Garstecki, M.J. Fuerstman, H.A. Stone \& G.M. Whitesides, Formation of droplets and bubbles in a microfluidic T-junction - Scaling and mechanism of break-up, Lab on a Chip Miniaturisation for Chemistry and Biology 6, 437-446 (2006).

71. J. Holman, Heat Transfer, (McGraw-Hill, 1981).

72. A.P. Sudarsan \& V.M. Ugaz, Multivortex micromixing, Proceedings of the National Academy of Sciences of the United States of America 103, 7228-7233 (2006).

73. A.L. Liu et al., Rapid method for design and fabrication of passive micromixers in microfluidic devices using a direct-printing process, Lab on a Chip - Miniaturisation for Chemistry and Biology 5, 974-978 (2005). 
74. C.S. Chen et al., Shrinky-Dink microfluidics: 3D polystyrene chips, Lab on a Chip Miniaturisation for Chemistry and Biology 8, 622-624 (2008).

75. T.M. Squires \& J.F. Brady, A simple paradigm for active and nonlinear microrheology, Physics of Fluids 17, 073101-073121 (2005).

76. M. Mitchell \& H.J.V. Tyrrell, Diffusion of benzene, phenol and resorcinol in propane-1,2-diol, and the validity of the Stokes-Einstein equation, Journal of the Chemical Society, Faraday Transactions 2: Molecular and Chemical Physics 68, 385-399 (1972).

77. A. Manz et al., Micromachining of monocrystalline silicon and glass for chemical analysis systems. A look into next century's technology or just a fashionable craze?, Trends in Analytical Chemistry 10, 144-149 (1991).

78. H. Jansen, H. Gardeniers, M. De Boer, M. Elwenspoek \& J. Fluitman, A survey on the reactive ion etching of silicon in microtechnology, Journal of Micromechanics and Microengineering $\mathbf{6}$, 14-28 (1996).

79. J. Xu, L. Locascio, M. Gaitan \& C.S. Lee, Room-temperature imprinting method for plastic microchannel fabrication, Analytical Chemistry 72, 1930-1933 (2000).

80. L. Martynova et al., Fabrication of Plastic Microfluid Channels by Imprinting Methods, Analytical Chemistry 69, 4783-4789 (1997).

81. J. Kameoka, H.G. Craighead, H. Zhang \& J. Henion, A polymeric microfluidic chip for CE/MS determination of small molecules, Analytical Chemistry 73, 1935-1941 (2001).

82. H. Becker \& U. Heim, Hot embossing as a method for the fabrication of polymer high aspect ratio structures, Sensors and Actuators, A: Physical 83, 130-135 (2000).

83. D.J. Harrison, A. Manz, Z. Fan, H. Ludi \& H.M. Widmer, Capillary electrophoresis and sample injection systems integrated on a planar glass chip, Analytical Chemistry 64, 1926-1932 (1992).

84. I. Rodriguez, P. Spicar-Mihalic, C.L. Kuyper, G.S. Fiorini \& D.T. Chiu, Rapid prototyping of glass microchannels, Analytica Chimica Acta 496, 205-215 (2003).

85. R.M. Lorenz, C.L. Kuyper, P.B. Allen, L.P. Lee \& D.T. Chiu, Direct laser writing on electrolessly deposited thin metal films for applications in micro- and nanofluidics, Langmuir 20, 1833-1837 (2004).

86. M.A. Roberts, J.S. Rossier, P. Bercier \& H. Girault, UV laser machined polymer substrates for the development of microdiagnostic systems, Analytical Chemistry 69, 2035-2042 (1997).

87. M.F. Jensen, M. Noerholm, L.H. Christensen \& O. Geschke, Microstructure fabrication with a $\mathrm{CO} 2$ laser system: Characterization and fabrication of cavities produced by raster scanning of the laser beam, Lab on a Chip - Miniaturisation for Chemistry and Biology 3, 302-307 (2003).

88. R.M. McCormick, R.J. Nelson, M.G. Alonso-Amigo, D.J. Benvegnu \& H.H. Hooper, Microchannel Electrophoretic Separations of DNA in Injection-Molded Plastic Substrates, Analytical Chemistry 69, 2626-2630 (1997).

89. H. Becker \& C. Gartner, Polymer microfabrication methods for microfluidic analytical applications, Electrophoresis 21, 12-26 (2000).

90. A. Bubendorfer, X. Liu \& A.V. Ellis, Microfabrication of PDMS microchannels using SU8/PMMA moldings and their sealing to polystyrene substrates, Smart Materials and Structures 16, 367-371 (2007). 
91. G.S. Fiorini, R.M. Lorenz, J. Kuo \& D.T. Chiu, Rapid prototyping of thermoset polyester microfluidic devices, Analytical Chemistry 76, 4697-4704 (2004).

92. R.K. Shah et al., Designer emulsions using microfluidics, Materials Today 11, 18-27 (2008).

93. G.M. Whitesides, E. Ostuni, S. Takayama, X. Jiang \& D.E. Ingber, Soft lithography in biology and biochemistry, Annual Review of Biomedical Engineering 3, 335-373 (2001).

94. S. Pannuthur, Flow control in microfluidics: are the workhorse flows adequate?, Lab on a Chip 8, 383-387 (2008).

95. P.H. Paul, M.G. Garguilo \& D.J. Rakestraw, Imaging of Pressure- And Electrokinetically Driven Flows through Open Capillaries, Analytical Chemistry 70, 2459-2467 (1998).

96. M.A. Day, The no-slip condition of fluid dynamics, Erkenntnis 33, 285-296 (1990).

97. C.H. Choi, K.J.A. Westin \& K.S. Breuer, presented at the American Society of Mechanical Engineers, Electronic and Photonic Packaging, EPP, New Orleans, LA, 2002.

98. J.J. Nagel, G. Mikhail, H. Noh \& J. Koo, presented at the Proceedings of SPIE - The International Society for Optical Engineering, San Diego, CA, 2006.

99. M.A. Unger, H.P. Chou, T. Thorsen, A. Scherer \& S.R. Quake, Monolithic microfabricated valves and pumps by multilayer soft lithography, Science 288, 113-116 (2000).

100. D.J. Laser \& J.G. Santiago, A review of micropumps, Journal of Micromechanics and Microengineering 14, 35-64 (2004).

101. P. Dutta, A. Beskok \& T.C. Warburton, Electroosmotic flow control in complex microgeometries, Journal of Microelectromechanical Systems 11, 36-44 (2002).

102. M. Madou et al., Lab on a CD, Annual Review of Biomedical Engineering 8, 601-628 (2006).

103. E. Berthier \& D.J. Beebe, Flow rate analysis of a surface tension driven passive micropump, Lab on a Chip - Miniaturisation for Chemistry and Biology 7, 1475-1478 (2007).

104. H. Moon, S.K. Cho, R.L. Garrell \& C.J. Kim, Low voltage electrowetting-on-dielectric, Journal of Applied Physics 92, 4080 (2002).

105. D. Huh et al., Gravity-driven microfluidic particle sorting device with hydrodynamic separation amplification, Analytical Chemistry 79, 1369-1376 (2007).

106. A. Wixforth, Acoustically driven planar microfluidics, Superlattices and Microstructures 33, 389-396 (2003).

107. A. Engel \& R. Friedrichs, On the electromagnetic force on a polarizable body, American Journal of Physics 70, 428-432 (2002).

108. K. Smistrup, Magnetic separation in microfluidic systems (PhD dissertation, Technical University of Denmark, 2007)

109. N. Pekas, M. Granger, M. Tondra, A. Popple \& M.D. Porter, Magnetic particle diverter in an integrated microfluidic format, Journal of Magnetism and Magnetic Materials 293, 584-588 (2005).

110. A.C. Siegel et al., Cofabrication of electromagnets and microfluidic systems in poly(dimethylsiloxane), Angewandte Chemie - International Edition 45, 6877-6882 (2006). 
111. S.S. Shevkoplyas, A.C. Siegel, R.M. Westervelt, M.G. Prentiss \& G.M. Whitesides, The force acting on a superparamagnetic bead due to an applied magnetic field, Lab on a Chip -

Miniaturisation for Chemistry and Biology 7, 1294-1302 (2007).

112. G. Friedman \& B. Yellen, Magnetic separation, manipulation and assembly of solid phase in fluids, Current Opinion in Colloid and Interface Science 10, 158-166 (2005).

113. N. Xia et al., Combined microfluidic-micromagnetic separation of living cells in continuous flow, Biomedical Microdevices 8, 299-308 (2006).

114. G.P. Hatch \& R.E. Stelter, Magnetic design considerations for devices and particles used for biological high-gradient magnetic separation (HGMS) systems, Journal of Magnetism and Magnetic Materials 225, 262-276 (2001).

115. C.H. Setchell, Magnetic separations in biotechnology - A review, Journal of chemical technology and biotechnology 35 B, 175-182 (1985).

116. T. Rheinlander, R. Kotitz, W. Weitschies \& W. Semmler, Magnetic fractionation of magnetic fluids, Journal of Magnetism and Magnetic Materials 219, 219-228 (2000).

117. J.H.P. Watson \& Z. Li, A study on mechanical entrapment in HGMS and vibration HGMS, Minerals Engineering 4, 815-823 (1991).

118. A. Jordan, T. Rheinlander, N. Waldofner \& R. Scholz, Increase of the specific absorption rate (SAR) by magnetic fractionation of magnetic fluids, Journal of Nanoparticle Research 5, 597$600(2003)$.

119. G. Glockl et al., The effect of field parameters, nanoparticle properties and immobilization on the specific heating power in magnetic particle hyperthermia, Journal of Physics Condensed Matter 18, 2935-2949 (2006).

120. P. Todd et al., Multistage magnetic particle separator, Journal of Magnetism and Magnetic Materials 225, 294-300 (2001).

121. U.O. Hafeli, M.A. Lobedann, J. Steingroewer, L.R. Moore \& J. Riffle, Optical method for measurement of magnetophoretic mobility of individual magnetic microspheres in defined magnetic field, Journal of Magnetism and Magnetic Materials 293, 224-239 (2005).

122. A. Sinha, R. Ganguly \& I.K. Puri, Magnetic separation from superparamagnetic particle suspensions, Journal of Magnetism and Magnetic Materials 321, 2251-2256 (2009).

123. C.H. Ahn, M.G. Allen, W. Trimmer, Y.N. Jun \& S. Erramilli, A fully integrated micromachined magnetic particle separator, Journal of Microelectromechanical Systems 5, 151-158 (1996).

124. J. Do, J.W. Choi \& C.H. Ahn, Low-cost magnetic interdigitated array on a plastic wafer, IEEE Transactions on Magnetics 40, 3009-3011 (2004).

125. Q. Ramadan, V. Samper, D. Poenar \& C. Yu, On-chip micro-electromagnets for magnetic-based bio-molecules separation, Journal of Magnetism and Magnetic Materials 281, 150-172 (2004).

126. Q. Ramadan, V. Samper, D. Poenar \& C. Yu, Magnetic-based microfluidic platform for biomolecular separation, Biomedical Microdevices 8, 151-158 (2006).

127. Q. Ramadan, V. Samper, D.P. Poenar \& C. Yu, An integrated microfluidic platform for magnetic microbeads separation and confinement, Biosensors and Bioelectronics 21, 1693-1702 (2006).

128. F.W. Osterberg et al., Bead Capture on Magnetic Sensors in a Microfluidic System, Sensors Journal, IEEE 9, 682-688 (2009). 
129. M. Bu, T.B. Christensen, K. Smistrup, A. Wolff \& M.F. Hansen, presented at the Transducers and Eurosensors '07 - 4th International Conference on Solid-State Sensors, Actuators and Microsystems, Lyon, 2007.

130. K. Smistrup et al., On-chip magnetic bead microarray using hydrodynamic focusing in a passive magnetic separator, Lab on a Chip - Miniaturisation for Chemistry and Biology 5, 1315-1319 (2005).

131. T. Deng, M. Prentiss \& G.M. Whitesides, Fabrication of magnetic microfiltration systems using soft lithography, Applied Physics Letters 80, 461 (2002).

132. T. Deng, G.M. Whitesides, M. Radhakrishnan, G. Zabow \& M. Prentiss, Manipulation of magnetic microbeads in suspension using micromagnetic systems fabricated with soft lithography, Applied Physics Letters 78, 1775-1777 (2001).

133. C. Bor Fuh \& S.Y. Chen, Magnetic split-flow thin fractionation: new technique for separation of magnetically susceptible particles, Journal of Chromatography A 813, 313-324 (1998).

134. N. Pamme \& A. Manz, On-chip free-flow magnetophoresis: Continuous flow separation of magnetic particles and agglomerates, Analytical Chemistry 76, 7250-7256 (2004).

135. M.A. Espy et al., An instrument for sorting of magnetic microparticles in a magnetic field gradient, Cytometry Part A 69, 1132-1142 (2006).

136. A.H. Latham, A.N. Tarpara \& M.E. Williams, Magnetic field switching of nanoparticles between orthogonal microfluidic channels, Analytical Chemistry 79, 5746-5752 (2007).

137. G. Blankenstein \& U.D. Larsen, Modular concept of a laboratory on a chip for chemical and biochemical analysis, Biosensors and Bioelectronics 13, 427-438 (1998).

138. S. Østergaard, G. Blankenstein, H. Dirac \& O. Leistiko, Novel approach to the automation of clinical chemistry by controlled manipulation of magnetic particles, Journal of Magnetism and Magnetic Materials 194, 156-162 (1999).

139. D.W. Inglis, R. Riehn, R.H. Austin \& J.C. Sturm, Continuous microfluidic immunomagnetic cell separation, Applied Physics Letters 85, 5093-5095 (2004).

140. K.H. Han \& A.B. Frazier, Paramagnetic capture mode magnetophoretic microseparator for high efficiency blood cell separations, Lab on a Chip - Miniaturisation for Chemistry and Biology 6, 265-273 (2006).

141. K.H. Man \& A. Bruno Frazier, Continuous magnetophoretic separation of blood cells in microdevlce format, Journal of Applied Physics 96, 5797-5802 (2004).

142. T. Lund-Olesen, H. Bruus \& M.F. Hansen, Quantitative characterization of magnetic separators: Comparison of systems with and without integrated microfluidic mixers, Biomedical Microdevices 9, 195-205 (2007).

143. R. Wirix-Speetjens \& J. De Boeck, On-chip magnetic particle transport by alternating magnetic field gradients, IEEE Transactions on Magnetics 40, 1944-1946 (2004).

144. A. Rida, V. Fernandez \& M.A.M. Gijs, Long-range transport of magnetic microbeads using simple planar coils placed in a uniform magnetostatic field, Applied Physics Letters 83, 23962398 (2003).

145. Q. Ramadan, C. Yu, V. Samper \& D.P. Poenar, Microcoils for transport of magnetic beads, Applied Physics Letters 88, 1-3 (2006). 
146. Q. Ramadan, V. Samper, D. Poenar \& Y.U. Chen, Evaluation of current-carrying wires for manipulation of magnetic micro/nanoparticles for biomedical applications, International Journal of Nanoscience 4, 489-499 (2005).

147. C.S. Lee, H. Lee \& R.M. Westervelt, Microelectromagnets for the control of magnetic nanoparticles, Applied Physics Letters 79, 3308-3310 (2001).

148. H. Lee, Y. Liu, D. Ham \& R.M. Westervelt, Integrated cell manipulation system CMOS/microfluidic hybrid, Lab on a Chip - Miniaturisation for Chemistry and Biology 7, 331337 (2007).

149. H. Lee, A.M. Purdon \& R.M. Westervelt, Manipulation of biological cells using a microelectromagnet matrix, Applied Physics Letters 85, 1063-1065 (2004).

150. H. Lee, A.M. Purdon \& R.M. Westervelt, Micromanipulation of biological systems with microelectromagnets, IEEE Transactions on Magnetics 40, 2991-2993 (2004).

151. J.C. McDonald et al., Fabrication of microfluidic systems in poly(dimethylsiloxane), Electrophoresis 21, 27-40 (2000).

152. B. Chooi, Development of a low-cost microfluidic process (Honours report, Victoria University, 2008)

153. Microsoft Visio 2003, Available at http://office.microsoft.com/en-us/visio/default.aspx.

154. MicroChem, SU-8 resists, Available at http://www.microchem.com/products/su_eight.htm.

155. M. Shaw, D. Nawrocki, R. Hurditch \& D. Johnson, Improving the process capability of SU-8, Microsystem Technologies 10, 1-6 (2003).

156. J. Zhang, K.L. Tan, G.D. Hong, L.J. Yang \& H.Q. Gong, Polymerization optimization of SU-8 photoresist and its applications in microfluidic systems and MEMS, Journal of Micromechanics and Microengineering 11, 20-26 (2001).

157. R. Feng \& R.J. Farris, Influence of processing conditions on the thermal and mechanical properties of SU8 negative photoresist coatings, Journal of Micromechanics and Microengineering 13, 80-88 (2003).

158. Processing Guidelines for SU-8 2100 and SU-8 2150, Available at http://www.microchem.com/products/pdf/SU-82000DataSheet2100and2150Ver5.pdf.

159. WS-400-6NPP-LITE Spin Coater, Available at http://www.laurell.com/product_detail.asp?Type=Lite $\& I D=1020$.

160. Edge Bead Remover datasheet, Available at http://www.microchem.com/products/pdf/ebr.pdf.

161. UV exposure units guide, Available at http://www.megauk.com/uv_exposure units.php.

162. Resist Thinners and Resist Removal, Available at http://www.microchem.com/products/ancillaries.htm.

163. J.C. McDonald \& G.M. Whitesides, Poly(dimethylsiloxane) as a material for fabricating microfluidic devices, Accounts of Chemical Research 35, 491-499 (2002).

164. J.N. Lee, C. Park \& G.M. Whitesides, Solvent Compatibility of Polydimethylsiloxane-Based Microfluidic Devices, Analytical Chemistry 75, 6544-6554 (2003). 
165. Information about Dow Corning ${ }^{\circledR}$ Brand Silicone Encapsulants, Available at http://www1.dowcorning.com/DataFiles/090007c88020bcca.pdf.

166. PVC Luer Barbed Adapters, Available at http://www.coleparmer.com/catalog/product view.asp?sku=4551000.

167. C.S. Effenhauser, G.J.M. Bruin, A. Paulus \& M. Ehrat, Integrated Capillary Electrophoresis on Flexible Silicone Microdevices: Analysis of DNA Restriction Fragments and Detection of Single DNA Molecules on Microchips, Analytical Chemistry 69, 3451-3457 (1997).

168. M.A. Eddings, M.A. Johnson \& B.K. Gale, Determining the optimal PDMS-PDMS bonding technique for microfluidic devices, Journal of Micromechanics and Microengineering 18, 067001-067005 (2008).

169. S. Satyanarayana, R.N. Karnik \& A. Majumdar, Stamp-and-stick room-temperature bonding technique for microdevices, Journal of Microelectromechanical Systems 14, $392-399$ (2005).

170. J.S. Go \& S. Shoji, A disposable, dead volume-free and leak-free in-plane PDMS microvalve, Sensors and Actuators, A: Physical 114, 438-444 (2004).

171. J. Kim, M.K. Chaudhury \& M.J. Owen, Hydrophobic recovery of polydimethylsiloxane elastomer exposed to partial electrical discharge, Journal of Colloid and Interface Science 226, 231-236 (2000).

172. K. Haubert, T. Drier \& D. Beebe, PDMS bonding by means of a portable, low-cost corona system, Lab on a Chip - Miniaturisation for Chemistry and Biology 6, 1548-1549 (2006).

173. A.F. Stalder, G. Kulik, D. Sage, L. Barbieri \& P. Hoffmann, A snake-based approach to accurate determination of both contact points and contact angles, Colloids and Surfaces A:

Physicochemical and Engineering Aspects 286, 92-103 (2006).

174. ImageJ image analysis software, Available at http://rsbweb.nih.gov/ij/.

175. H. Hillborg et al., Crosslinked polydimethylsiloxane exposed to oxygen plasma studied by neutron reflectometry and other surface specific techniques, Polymer 41, 6851-6863 (2000).

176. Frenergy Magnets - Disc $2 \mathrm{~mm}$ dia. $x$ 1mm height, Available at http://www.frenergy.com.au/index.php?act=viewProd\&productId=39.

177. Metglas 2826MB Technical Bulletin, Available at http://www.metglas.com/downloads/2826mb.pdf.

178. Metglas 2826MB Material Safety Data Sheet, Available at http://metglas.com/downloads/msds/msds2826mb.pdf.

179. R. Hasegawa, Applications of amorphous magnetic alloys, Materials Science and Engineering A 375-377, 90-97 (2004).

180. S. Cheong, Personal communication.

181. P. Ferguson, Personal communication, (2009).

182. NE-1000 Single Syringe Pump, Available at http://www.syringepump.com/NE-1000.htm.

183. Flexible tubing and C-FLEX Tubing, Available at http://www.coleparmer.com/catalog/product_view.asp?sku=0642462\#tech. 
184. PTFE Color-Coded Tubing and Green Tubing, Available at http://www.coleparmer.com/catalog/product_view.asp?sku=9613042.

185. S. Foner, Versatile and Sensitive Vibrating-Sample Magnetometer, Review of Scientific Instruments 30, 548-557 (1959).

186. Vibrating Sample Magnetometer, Available at http://www.lakeshore.com/sys/vsm/vsmm.html.

187. McElfresh, (Quantum Design, Inc., San Diego, 1994), pp. 34.

188. Quantum Design Homepage, Available at http://www.qdusa.com/.

189. Fundamentals of Magnetism and Magnetic Measurements, Available at http://www.qdusa.com/resources/pdf/FundPrimer.pdf.

190. Carl Roth homepage, Available at http://www.carl-roth.de.

191. Queen Fine Foods - Food colouring product range, Available at http://www.queenessences.com.au/products/show.php?categoryid=3.

192. Polysciences, Polybead Microspheres Datasheet, Available at http://www.polysciences.com/SiteData/docs/TDS\%20788/fa3c300bf743114f6efc0b6b377e6ec4/ TDS\%20788.pdf.

193. Invitrogen, Dynabeads MyOne Carboxylic Acid Manual, Available at http://tools.invitrogen.com/content/sfs/manuals/650.11 12 13 Dynabeads MyOne Carboxylic Acid_rev004).pdf.

194. Spherotech, Paramagnetic particles catalogue, Available at http://www.spherotech.com/para_par.htm.

195. C. Johansson, M. Hanson, M.S. Pedersen \& S. Mørup, Magnetic properties of magnetic liquids with iron-oxide particles - The influence of anisotropy and interactions, Journal of Magnetism and Magnetic Materials 173, 5-14 (1997).

196. J.J. Lu, H.Y. Deng \& H.L. Huang, Thermal relaxation of interacting fine magnetic particles field-cooled and zero-field-cooled magnetization variation, Journal of Magnetism and Magnetic Materials 209, 37-41 (2000).

197. T. Sugimoto, Monodispersed Particles, (Elsevier Science, 2001).

198. ProSciTech, ProSciTech: Page Q1: Latex Spheres for Calibration, Cytology and Immunology \& Magnetic Separators, Available at

http://www.proscitech.com.au/cataloguex/online.asp?page=q1\#qpm-20-10, (2009).

199. M.B. Chan-Park, J. Zhang, Y. Yan \& C.Y. Yue, Fabrication of large SU-8 mold with high aspect ratio microchannels by UV exposure dose reduction, Sensors and Actuators B: Chemical 101, 175-182 (2004).

200. Y.J. Chuang, F.G. Tseng \& W.K. Lin, Reduction of diffraction effect of UV exposure on SU-8 negative thick photoresist by air gap elimination, Microsystem Technologies 8, 308-313 (2002).

201. T. Kajishima \& S. Takiguchi, Interaction between particle clusters and particle-induced turbulence, International Journal of Heat and Fluid Flow 23, 639-646 (2002).

202. A.R. Abate, D. Lee, T. Do, C. Holtze \& D.A. Weitz, Glass coating for PDMS microfluidic channels by sol-gel methods, Lab on a Chip - Miniaturisation for Chemistry and Biology 8, 516518 (2008). 
203. Cole-Parmer: Chemical Resistance Database, Available at

http://www.coleparmer.com/techinfo/ChemComp.asp, (2009).

204. Nanoport Assemblies, Available at http://www.upchurch.com/PDF/I-Cards/N4.PDF.

205. J. Greener, W. Li, D. Voicu \& E. Kumacheva, Chips \& Tips: Reusable, robust NanoPort connections to PDMS chips, Available at http://www.rsc.org/Publishing/Journals/lc/Chips_and_Tips/nanoport_connections.asp.

206. H. Makamba, J.H. Kim, K. Lim, N. Park \& J.H. Hahn, Surface modification of poly(dimethylsiloxane) microchannels, Electrophoresis 24, 3607-3619 (2003).

207. L.E.M. Gevers, I.F.J. Vankelecom \& P.A. Jacobs, Solvent-resistant nanofiltration with filled polydimethylsiloxane (PDMS) membranes, Journal of Membrane Science 278, 199-204 (2006). 